Aus der Klinik für Psychosomatische Medizin und Psychotherapie (Prof. Dr. med. Ch. Herrmann-Lingen)

der Medizinischen Fakultät der Universität Göttingen

\title{
Verlauf der Stressreagibilität \\ bei Patientinnen mit komplexen \\ Traumafolgestörungen
}

\author{
INAUGURAL-DISSERTATION \\ zur Erlangung des Doktorgrades \\ der Medizinischen Fakultät der \\ Georg-August-Universität zu Göttingen
}

vorgelegt von

Frauke Seutemann

aus

Hameln

Göttingen 2019 
Dekan:

Referent

Ko-Referentin:

Promotor-Vertreterin:

Datum der mündlichen Prüfung:
Prof. Dr. rer. nat. H.K. Kroemer

Prof. Dr. med. Ch. Herrmann-Lingen

Prof. Dr. rer. nat. A. Rodenbeck

Prof. Dr. hum. biol. M. Schön

09.12.2020 
Hiermit erkläre ich, die Dissertation mit dem Titel "Verlauf der Stressreagibilität bei Patientinnen mit komplexen Traumafolgestörungen" eigenständig angefertigt und keine anderen als die von mir angegebenen Quellen und Hilfsmittel verwendet zu haben.

Göttingen, den 


\section{Inhaltsverzeichnis}

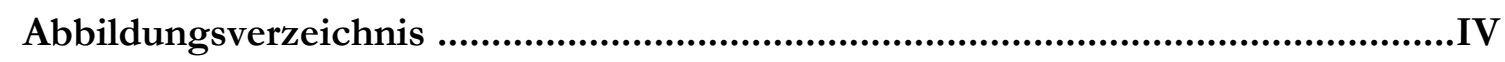

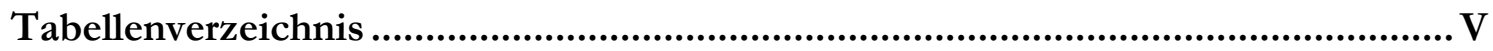

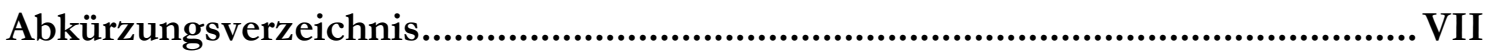

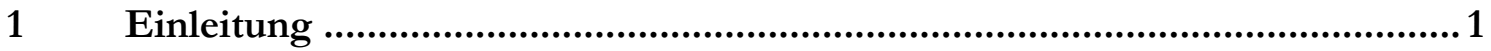

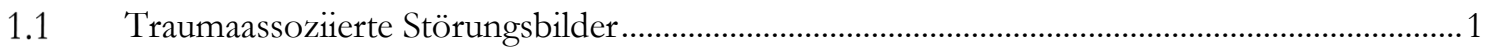

1.1.1 Die Posttraumatische Belastungsstörung ……….....................................................................

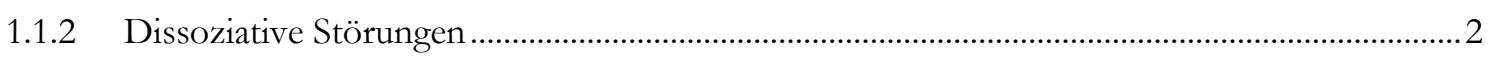

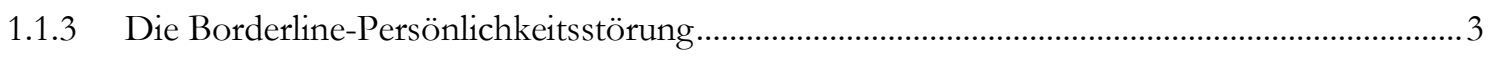

1.1.4 Komplexe Traumafolgestörungen.....................................................................................

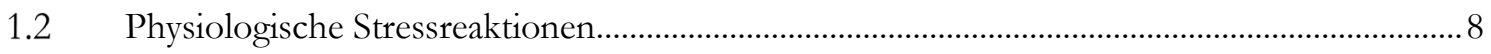

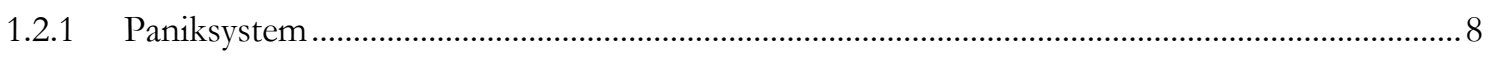

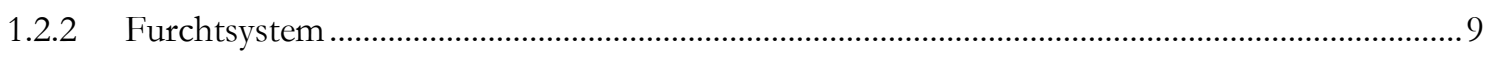

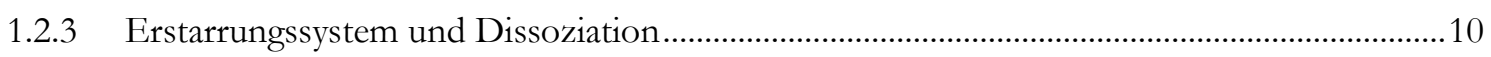

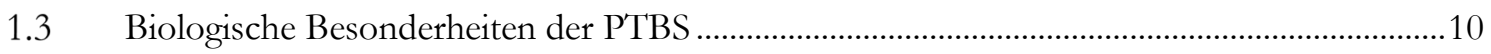

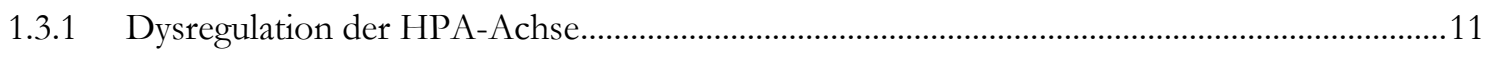

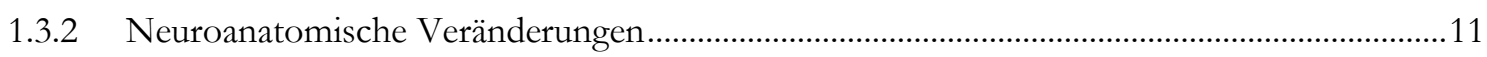

1.3.3 Dysregulation des autonomen Nervensystems.......................................................................12

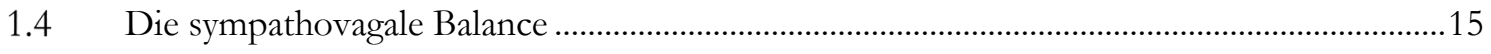

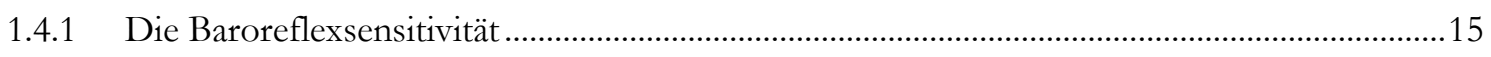

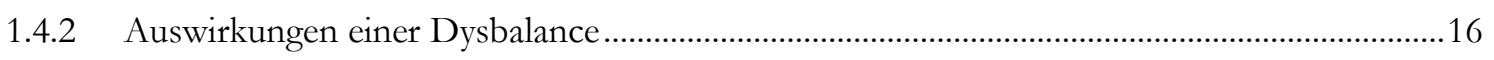

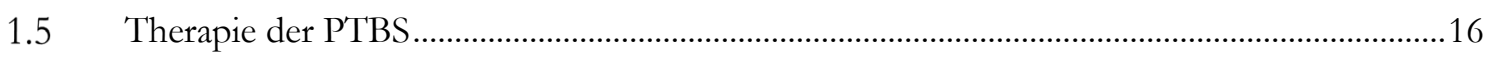

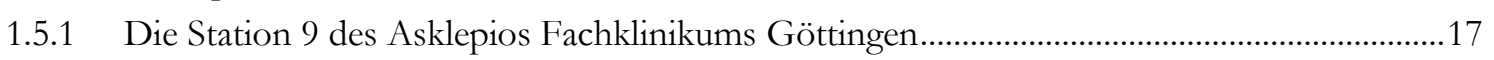

1.5.2 Therapiewirkung auf die autonome Dysregulation...............................................................

2 Ziele und Hypothesen ............................................................................. 19

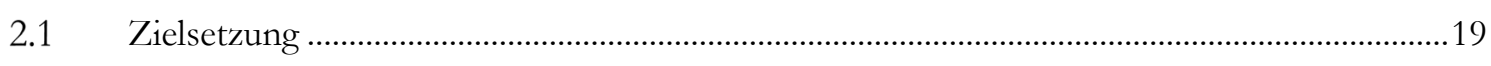

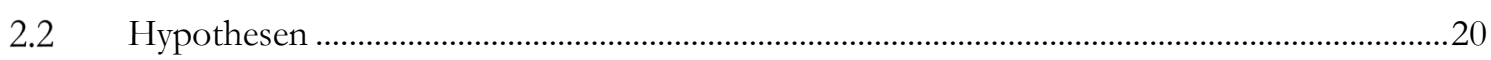

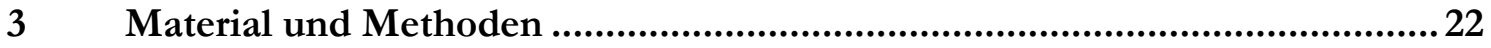

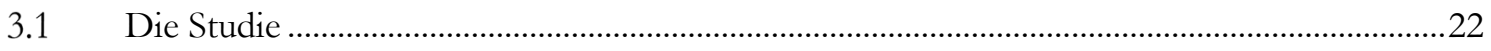

3.1.1 Die Stichprobe: Rekrutierung, Ein- und Ausschlusskriterien .................................................2

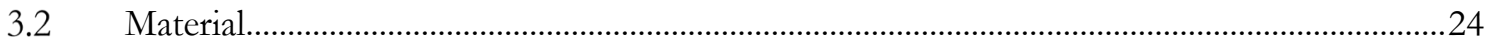

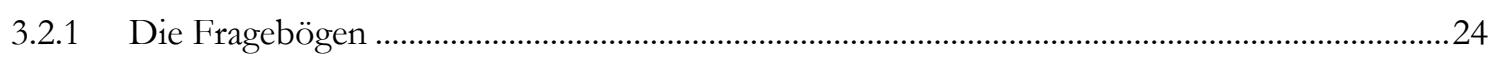

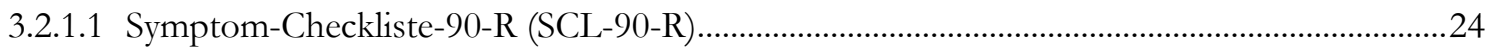

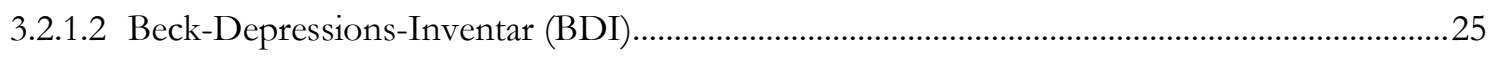

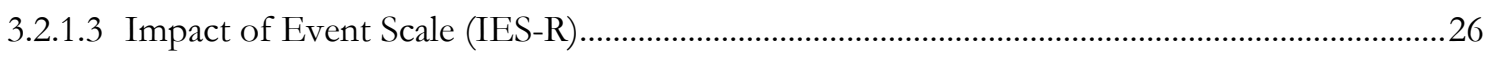

3.2.1.4 Fragebogen zu dissoziativen Symptomen (FDS) ……………………………………........26

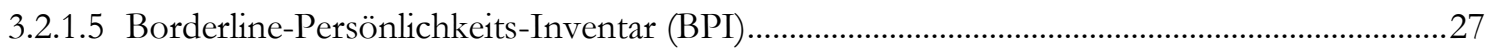

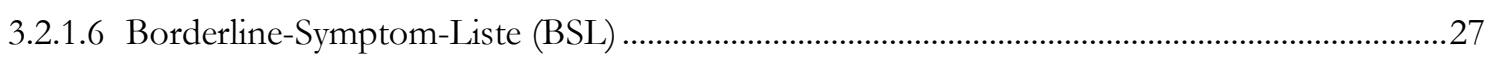

3.2.1.7 Allgemeine Fragebögen und Erhebung klinischer Daten......................................................28

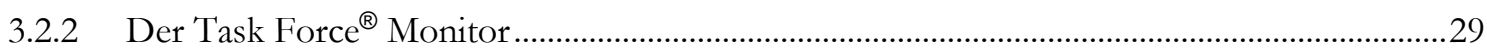




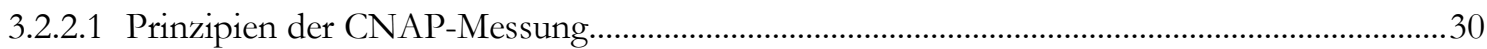

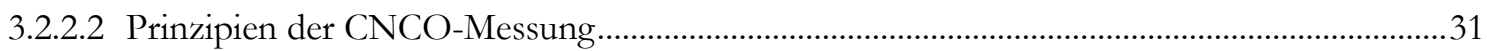

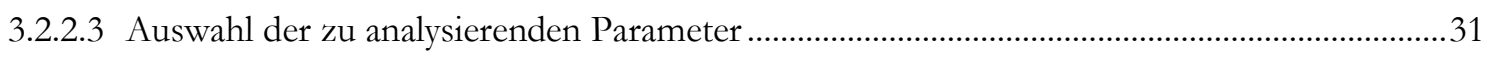

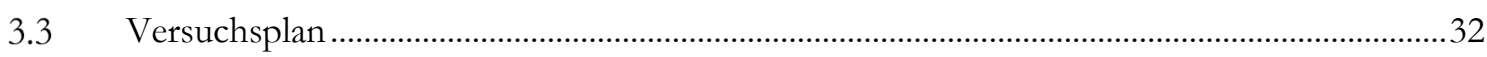

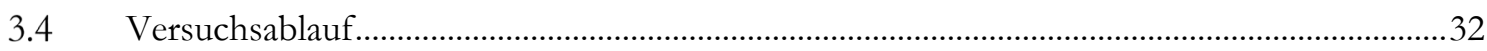

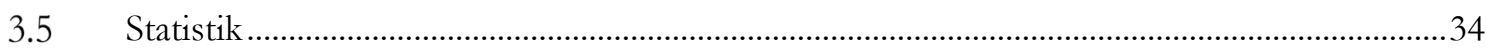

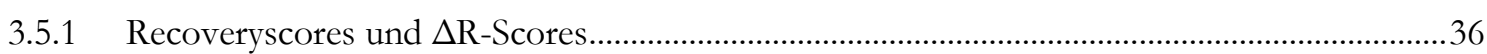

$4 \quad$ Ergebnisse........................................................................................ 38

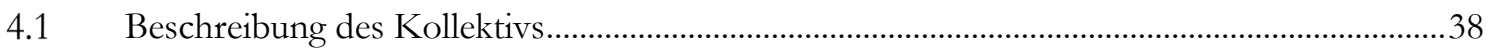

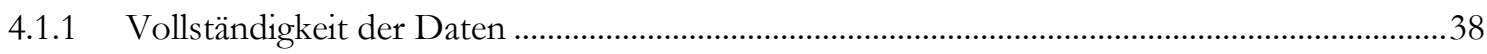

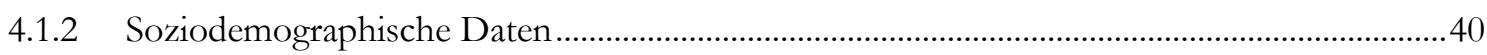

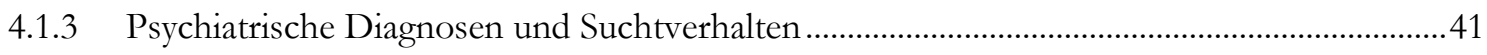

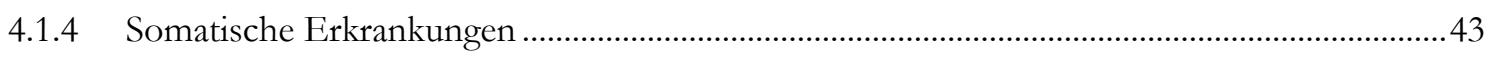

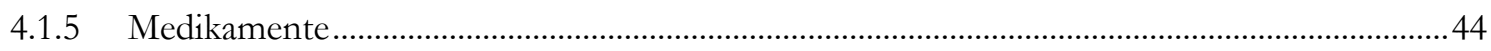

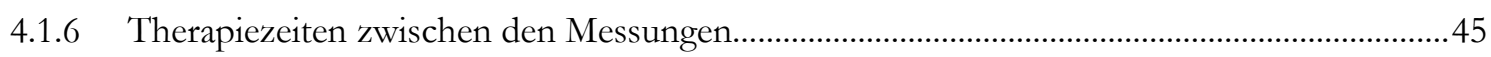

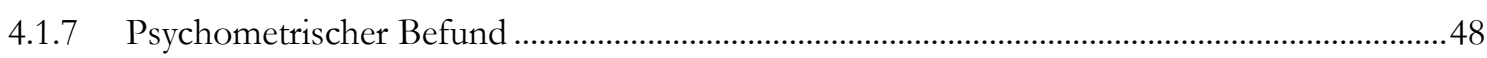

4.2 Prüfung von Voraussetzungen für die statistische Auswertung ...............................................52

4.2.1 Zeitspanne zwischen Baseline und Follow-up............................................................................52

4.2.2 Vergleich der Gruppe „Follow-up“ mit der Gruppe „Nur Baseline“ ......................................52

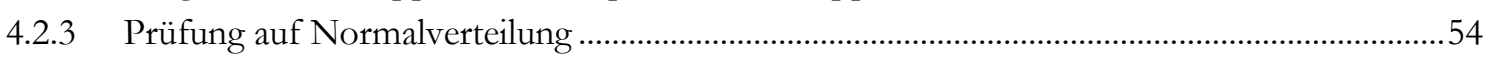

4.2.4 Bezugsgrößen für die Stress reagibilität ....................................................................................5

4.2.5 Graphische Betrachtung der $\Delta$ R-Scores zur Detektion von Extremwerten ...........................56

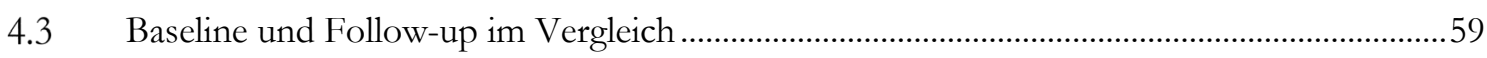

4.3.1 Veränderungen im Stressempfinden (Hypothese 1) ..............................................................59

4.3.2 Veränderungen im psychometrischen Befund (Hypothese 2) ..............................................61

4.3.3 Deskriptiver Vergleich der TFM-Daten über die Phasen........................................................63

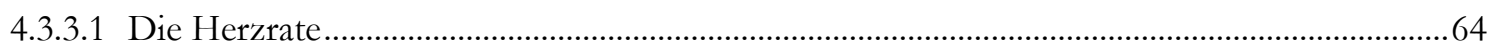

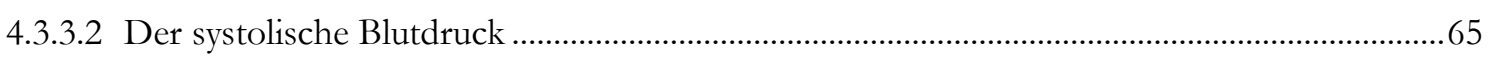

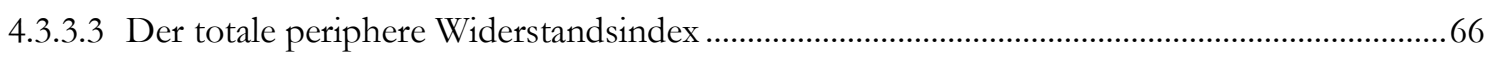

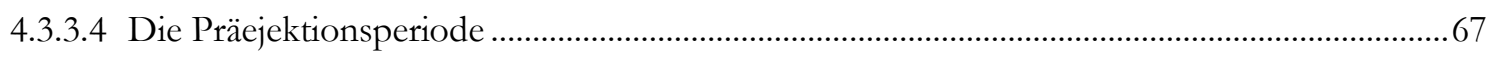

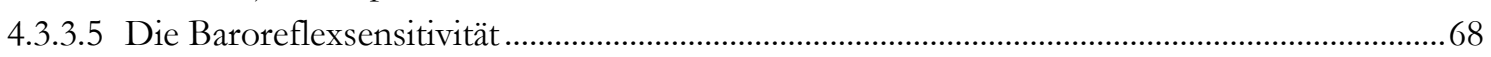

4.3.4 Kardiovaskuläre Ruheaktivierung zu den Messzeitpunkten (Hypothese 3a).........................69

4.3.5 Reactivity: Aktivierung durch die Stressoren zu den Messzeitpunkten..................................69

4.3.6 Recovery: Erholung nach der Stressreaktion zu den Messzeitpunkten..................................72

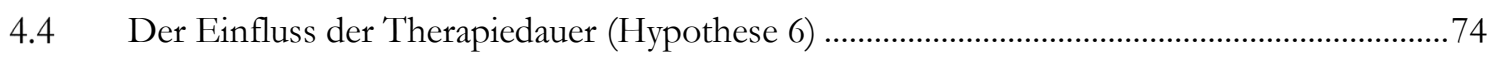

4.5 Die Reagibilität und mögliche Einflussfaktoren...............................................................76

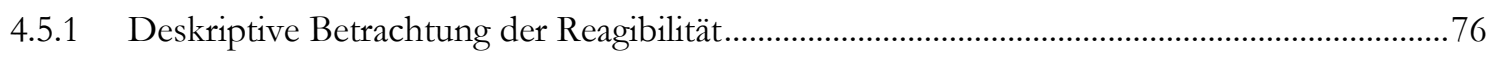

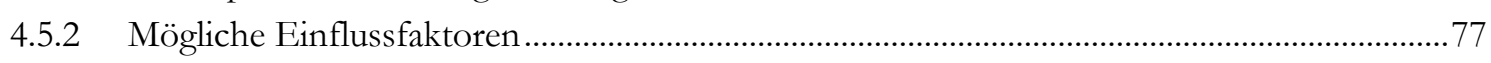




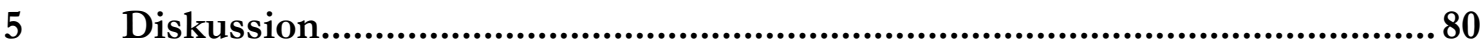

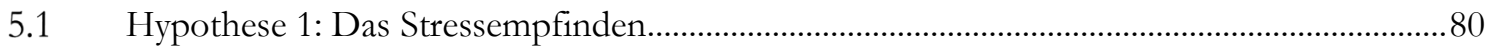

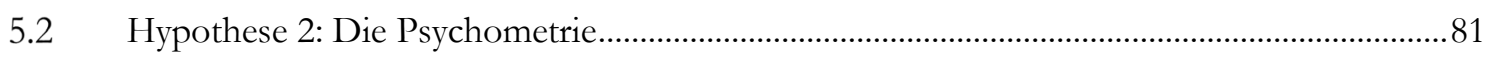

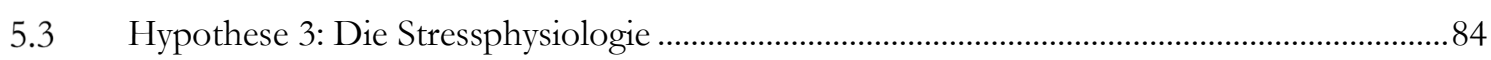

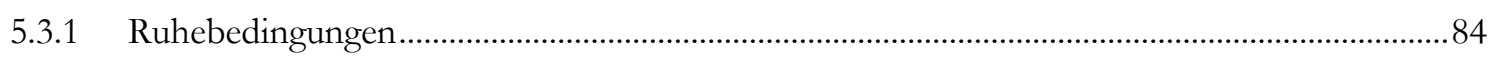

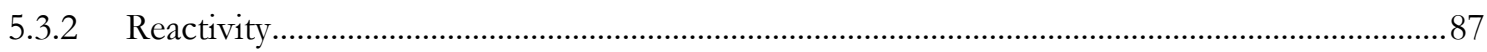

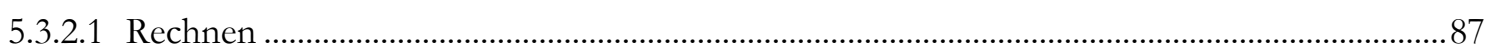

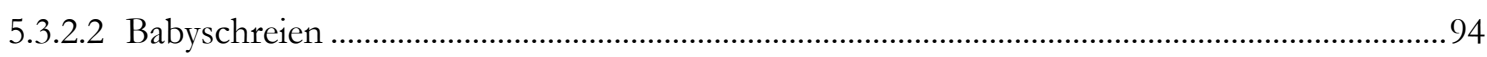

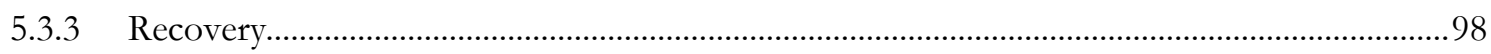

5.3.4 Allgemeine Stressreagibilität sowie Charakteristika der einzelnen Parameter ........................103

$5.4 \quad$ Hypothese 4 bis 6: Wechselwirkungen zwischen Psyche, Physiologie und Therapie ...........106

5.5 Psychophysiologische Evaluation der Therapiewirkung.............................................................108

$6 \quad$ Neue Forschungsansätze und Limitationen ...........................................117

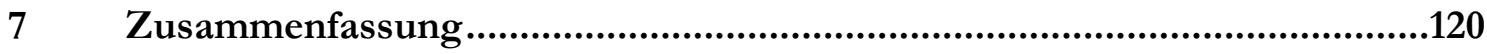

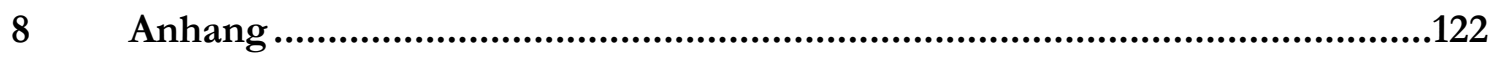

8.1 Tabellen zur Testung auf systematische Unterschiede zwischen den Gruppen „Followup“ und „Nur Baseline “.........................................................................................................122

8.2 Vergleichstabellen: Reactivity und Recovery ohne Ausschluss der Patientin E1...................125

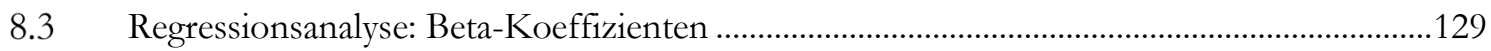

8.4 Vergleichstabelle: Tabelle 21 ohne Ausschluss der Patientin E1.............................................131

8.5 Vergleichstabelle: Tabelle 21 mit Ausschluss der Patientin E2...............................................132

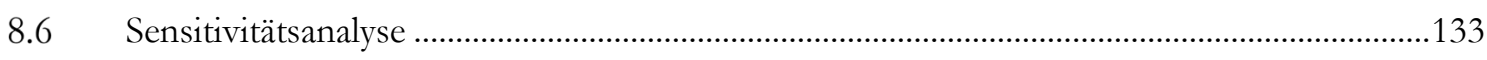

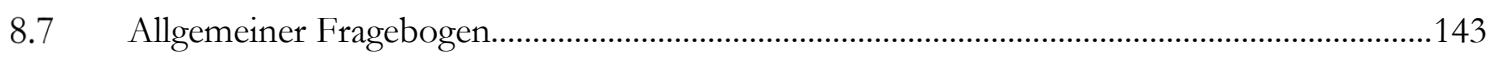

8.8 Auszug aus dem Fragebogen zu Therapien und Medikation.....................................................145

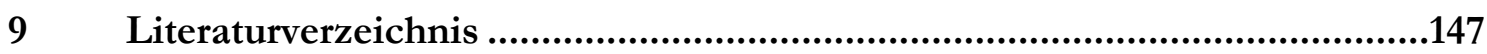




\section{Abbildungsverzeichnis}

Abbildung 1: Messphasen während der Task Force Monitor ${ }^{\circledR-M e s s u n g ~}$ 34

Abbildung 2: Formel zur Berechnung der $\Delta \mathrm{R}$-Scores am Beispiel Herzrate.

Abbildung 3: Stationäre Psychotherapie der Follow-up-Teilnehmerinnen auf der Station 9 des

Asklepios Fachklinikums Göttingen zwischen 2009 und 2013

Abbildung 4: Stationäre Therapie der Follow-up-Teilnehmerinnen auf einer psychiatrischen oder

psychotherapeutischen Station zwischen 2009 und 2013 ...............................................46

Abbildung 5: Ambulante psychotherapeutische Behandlungen der Follow-up-Teilnehmerinnen

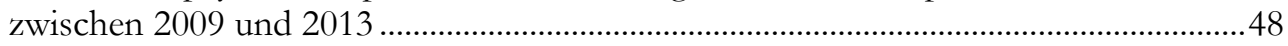

Abbildung 6: Vergleich der Gruppe „Follow-up“ mit der Gruppe „Nur Baseline“...............................53

Abbildung 7: Score $\Delta$ R-sBP-Babyschreien als Boxplot dargestellt .........................................................57

Abbildung 8: Score $\Delta$ R-TPRI-Babyschreien als Boxplot dargestellt .......................................................57

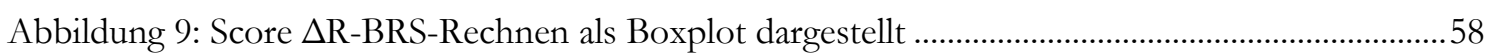

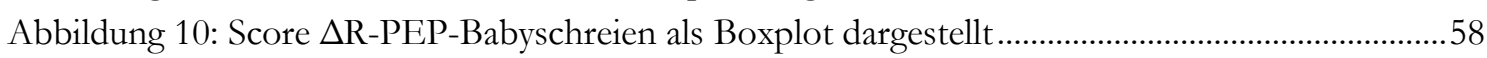

Abbildung 11: Stressempfinden in den verschiedenen Messphasen ......................................................59

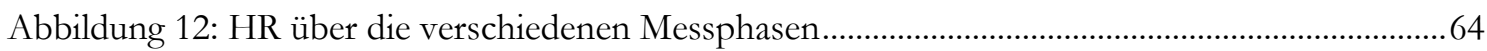

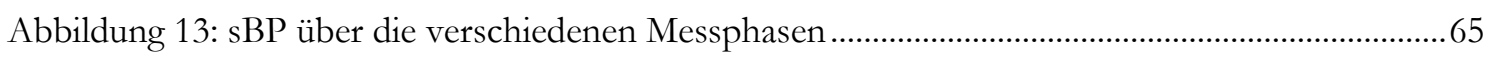

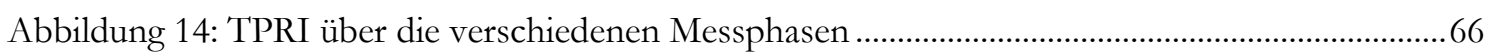

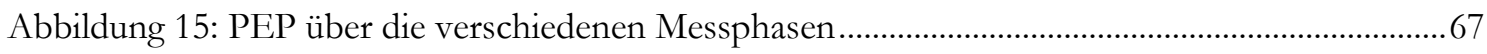

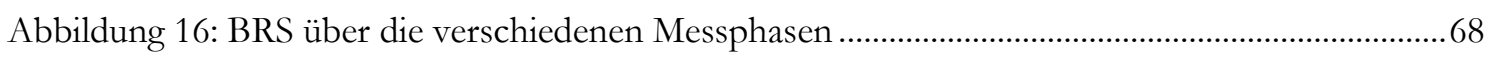

Abbildung 17: Streudiagramm zur Korrelation zwischen dem Stressempfinden in der Phase

Ruhe 1 (Baseline) und der Variable „Therapiedauer Station 9“.......................................76

Abbildung 18: Streudiagramm zur Korrelation zwischen der Differenz des BDI (Baseline Follow-up) und dem Score $\Delta$ R-HR-Rechnen.................................................................79 


\section{Tabellenverzeichnis}

Tabelle 1: Übersicht zu den fehlenden Daten und den davon betroffenen Analysen ........................38

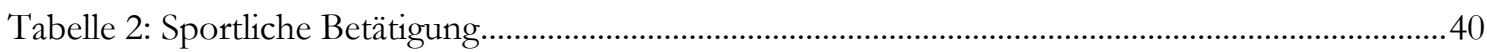

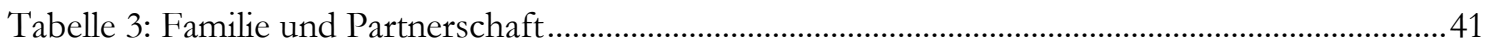

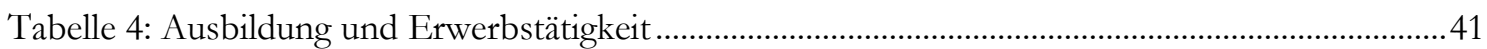

Tabelle 5: Überblick über die somatischen Erkrankungen ..................................................................43

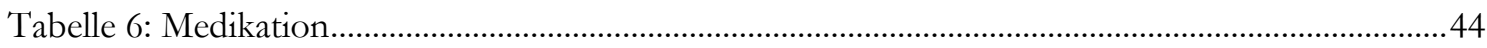

Tabelle 7: Subskalen und Globale Kennwerte der SCL-90-R mit T-Werten.......................................48

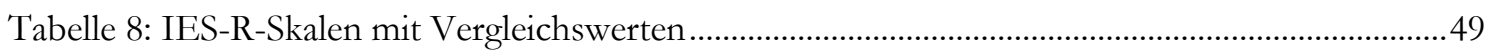

Tabelle 9: FDS- und DES-Gesamtskalenwerte sowie FDS-Subskalen ................................................50

Tabelle 10: FDS- und DES-Vergleichsmittelwerte verschiedener Stichproben..................................50

Tabelle 11: BPI-Skalen und BPI-Cut-20 mit T-Werten und Prozenträngen ......................................51

Tabelle 12: BSL-Subskalen und BSL-Gesamtskala mit Prozenträngen.................................................52

Tabelle 13: „Ruhe“-ANOVA. Messwerte der Phasen Ruhe 1 und Entspannungsmusik (Baseline und Follow-up) mit einer 2 x 2 ANOVA für Messwiederholung...................................55

Tabelle 14: Vergleich von Baseline und Follow-up bzgl. der psychometrischen Fragebögen............61

Tabelle 15: Reactivity Rechnen. Messwerte der Phasen Ruhe 1 und Rechnen (Baseline und Follow-up) mit einer 2 x 2 ANOVA für Messwiederholungen .......................................70

Tabelle 16: Reactivity Babyschreien. Messwerte der Phasen Ruhe 1 und Babyschreien (Baseline und Follow-up) mit einer 2 x 2 ANOVA für Messwiederholungen ..............................71

Tabelle 17: Recovery Rechnen. Messwerte der Phasen Rechnen und Musik (Baseline und Followup) mit einer 2 x 2 ANOVA für Messwiederholungen...................................................72

Tabelle 18: Recovery Babyschreien. Messwerte der Phasen Babyschreien und Musik (Baseline und Follow-up) mit einer 2 x 2 ANOVA für Messwiederholungen .....................................73

Tabelle 19: Korrelationen zwischen den Variablen zur Therapiedauer und den Variablen zum Stressempfinden und zum psychometrischen Befund der Baseline-Untersuchung.....75

Tabelle 20: Recoveryscores und $\Delta \mathrm{R}$ Rechnen- und $\Delta \mathrm{R}$ Babyschreien-Scores ...................................77

Tabelle 21: Korrelationen zwischen den Scores $\Delta \mathrm{R}$ Rechnen und $\Delta \mathrm{R}$ Babyschreien und den Variablen zu Stressempfinden, Psychometrie und Therapiedauer .................................78

Tabelle A1: Unabhängiger t-Test: Frage nach systematischen Unterschieden zwischen den Gruppen „Nur Baseline“ und „Follow-up“

Tabelle A2: Chi-Quadrat-Test: Frage nach systematischen Unterschieden zwischen den Gruppen „Nur Baseline“ und „Follow-up“

Tabelle A3: Chi-Quadrat-Test: Frage nach systematischen Unterschieden zwischen den Gruppen „Nur Baseline“ und „Follow-up“ (mit Zusammenfassung von Kategorien) ..............124

Tabelle A4: Vergleich zur Tabelle 15, ohne Ausschluss von Patientin E1 ........................................125

Tabelle A5: Vergleich zur Tabelle 16, ohne Ausschluss von Patientin E1 ......................................126

Tabelle A6: Vergleich zur Tabelle 17, ohne Ausschluss von Patientin E1 ......................................127

Tabelle A7: Vergleich zur Tabelle 18, ohne Ausschluss von Patientin E1 .......................................128

Tabelle A8: Koeffizienten der Regressionsanalyse „Therapiedauer gesamt“ ...................................129

Tabelle A9: Koeffizienten der Regressionsanalyse „Therapiedauer Station 9“.................................130

Tabelle A10: Vergleich zur Tabelle 21, ohne Ausschluss der Patientin E1 ......................................131

Tabelle A11: Vergleich zur Tabelle 21, mit Ausschluss der Patientin E1 und der Patientin E2 2.....132

Tabelle A12: Effektstärken ergänzend zur Tabelle 13 für die Sensitivitätsanalyse ............................133

Tabelle A13: Vergleich zur Tabelle 13, Ausschluss der drei Patientinnen mit Wechsel in der Betablockermedikation (Sensitivitätsanalyse), mit Effektstärken 
Tabelle A14: Abhängige t-Tests: Vergleich Ruhe 1 Baseline und Ruhe 1 Follow-up für HR, sBP, TPRI und PEP. Ergänzt durch Effektstärken für die Sensitivitätsanalyse

Tabelle A15: Wilcoxon-Vorzeichen-Rang-Test: Vergleich Ruhe 1 Baseline und Ruhe 1 Follow-up für die BRS. Ergänzt durch Effektstärken für die Sensitivitätsanalyse.

Tabelle A16: Abhängige t-Tests: Vergleich Ruhe 1 Baseline und Ruhe 1 Follow-up für HR, sBP, TPRI und PEP, Ausschluss der drei Patientinnen mit Wechsel in der Betablockermedikation (Sensitivitätsanalyse), mit Effektstärken

Tabelle A17: Wilcoxon-Vorzeichen-Rang-Test: Vergleich Ruhe 1 Baseline und Ruhe 1 Follow-up für die BRS, Ausschluss der drei Patientinnen mit Wechsel in der Betablockermedikation (Sensitivitätsanalyse), mit Effektstärken

Tabelle A18: Vergleich zur Tabelle 15, Ausschluss der drei Patientinnen mit Wechsel in der Betablockermedikation (Sensitivitätsanalyse), mit Effektstärken

Tabelle A19: Vergleich zur Tabelle 16, Ausschluss der drei Patientinnen mit Wechsel in der Betablockermedikation (Sensitivitätsanalyse), mit Effektstärken

Tabelle A20: Effektstärken ergänzend zur Tabelle 15 für die Sensitivitätsanalyse 139

Tabelle A21: Effektstärken ergänzend zur Tabelle 16 für die Sensitivitätsanalyse

Tabelle A22: Vergleich zur Tabelle 17, Ausschluss der drei Patientinnen mit Wechsel in der Betablockermedikation (Sensitivitätsanalyse), mit Effektstärken

Tabelle A23: Vergleich zur Tabelle 18, Ausschluss der drei Patientinnen mit Wechsel in der Betablockermedikation (Sensitivitätsanalyse), mit Effektstärken

Tabelle A24: Effektstärken ergänzend zur Tabelle 17 für die Sensitivitätsanalyse 142

Tabelle A25: Effektstärken ergänzend zur Tabelle 18 für die Sensitivitätsanalyse 


\section{Abkürzungsverzeichnis}

ACTH

ANOVA

ANS

ASS

BDI

BPI

BPS

BRS

BSA

BSL

CI

CNAP

$\mathrm{CNCO}$

$\mathrm{CRH}$

dBP

DBT

DDNOS

DES

DESNOS

DSM-III-R

DSM-IV

DSM-V

DTE

$\mathrm{EKG}$

EMDR

EMG

FDS

GR

GSI

HF-HRV

HPA

HR

HRV

$\mathrm{HZV}$

ICD-10

IES-R

IKG

IL-6
Adrenocorticotropes Hormon

Analysis of $V$ ariance

Autonomes Nervensystem

Acetylsalicylsäure

Beck-Depressions-Inventar

Borderline-Persönlichkeits-Inventar

Borderline-Persönlichkeitsstörung

Baroreflexsensitivität

Body Surface Area

Borderline-Symptom-Liste

Cardiac Index

Continuous Non-invasive Arterial Pressure (Task Force ${ }^{\circledR}$ Monitor)

Continuous Non-invasive Cardiac Output and stroke volume (Task Force $®$ Monitor)

Corticotropin-releasing hormone

diastolischer Blutdruck (diastolic Blood Pressure)

Dialektisch-Behaviorale Therapie

Dissociative Disorder Not Otherwise Specified

Dissociative Experience Scale

Disorders of Extreme Stress Not Otherwise Specified

Diagnostic and Statistical Manual of Mental Disorders (3 ${ }^{\text {rd }}$ edition, revision)

Diagnostic and Statistical Manual of Mental Disorders (4 $4^{\text {th }}$ edition)

Diagnostic and Statistical Manual of Mental Disorders (5 th edition)

Direct Therapeutic Exposure

Elektrokardiogramm

Eye Movement Desensitization and Reprocessing

Elektromyographie

Fragebogen zu dissoziativen Symptomen

Glukokortikoid-Rezeptoren

Global Severity Index (SCL-90-R)

High Frequencies-Herzratenvariabilität

Hypothalamus - Hypophyse (pituitary gland) - Nebennierenrinde (adrenal glands)

Herzrate

Herzratenvariabilität

Herzzeitvolumen

International Statistical Classification of Diseases and Related Health Problems (10. Revision )

Impact of Event Scale-Revised

Impedanzkardiographie

Interleukin-6 
kPTBS komplexe Posttraumatische Belastungsstörung

kVT kognitive Verhaltenstherapie

LF/HF Low Frequencies/High Frequencies

$\mathrm{M}$

Mittelwert

n.s. nicht signifikant

NSAR Nichtsteroidales Antirheumatikum

PEP Präejektionsperiode

PITT Psychodynamisch Imaginative Traumatherapie

PSDI Positive Symptom Distress Index (SCL-90-R)

PST

Positive Symptom Total (SCL-90-R)

PTBS Posttraumatische Belastungsstörung

$\mathrm{R}$

Recoveryscore Rechnen bzw. Babyschreien

REM

Rapid Eye Movement

RMSSD

Root Mean Square of the Successive Differences

RSA

Respiratory Sinus Arrbythmia

$\mathrm{sBP}$

systolischer Blutdruck (systolic Blood Pressure)

SCL-90-R Symptom-Checkliste-90-R

$\mathrm{SD}$

Standard Deviation

sPT

supportive Psychotherapie

SSNRI

Selective Serotonin-Noradrenalin-Reuptake-Inbibitor (Selektive SerotoninNoradrenalin-Wiederaufnahme-Hemmer)

SSRI

Selective Serotonin Reuptake Inbibitor (Selektive Serotonin-WiederaufnahmeHemmer)

TFM Task Force ${ }^{\circledR}$ Monitor

THC Tetrahydrocannabinol

TPRI

W

Totaler peripherer Widerstandsindex (Total Peripheral Resistance Index)

$\Delta \mathrm{R}$

Warteliste

$\Delta$ R-Score Rechnen bzw. Babyschreien 


\section{$1 \quad$ Einleitung}

In der vorliegenden Arbeit wird die kardiovaskuläre Stressreagibilität von Patientinnen mit komplexen Traumafolgestörungen vor und nach Therapie verglichen. Die folgende Einleitung stellt zunächst den aktuellen Forschungsstand zu dieser Thematik dar, bevor die Zielsetzung und Fragestellung der Arbeit sowie im Kapitel „Material und Methoden“ Ablauf und Inhalt der Studie genauer erläutert werden.

\subsection{Traumaassoziierte Störungsbilder}

Gerät ein Mensch einmal oder auch mehrmals in eine belastende Situation, in der er Entsetzen, intensive Angst, tiefe Verzweiflung und Hilflosigkeit empfindet, kann das Erlebte auf ihn traumatisierend wirken. Potenziell traumatisierende Ereignisse sind z. B. Kriegshandlungen, körperliche wie psychische Gewalt, Vergewaltigungen, sexueller Missbrauch und Vernachlässigungen, aber auch Naturkatastrophen, Unfälle oder die Diagnose einer schwerwiegenden Erkrankung. Zur Verursachung eines solchen psychischen Traumas, einer ,seelischen Wunde“ (Schellong 2013, S. 42), muss das Ereignis nicht zwingend direkt die eigene Person betreffen, sondern kann auch aus der Beobachtung, wie dieses einer anderen Person widerfährt (z. B. als Zeuge einer Gewalttat, eines Unfalls), resultieren (Flatten et al. 2013).

Die wohl bekannteste mögliche Folgereaktion auf ein Trauma ist die Posttraumatische Belastungsstörung (PTBS). Aber auch andere Störungsbilder scheinen eng mit Traumatisierungen assoziiert zu sein. So finden sich dissoziative Zustände besonders häufig bei Traumatisierten sowie PTBS-Patienten. Und aufgrund der hohen Trauma-Prävalenz bei Patienten mit einer Borderline-Persönlichkeitsstörung (BPS) wird vermutet, dass Traumatisierungen ätiologisch an der Herausbildung dieser Persönlichkeitsstörung beteiligt sein können. In der klinischen Beobachtung wird nach schwerwiegenden kindlichen Traumatisierungen häufig eine charakteristische Symptomatik beschrieben, die Merkmale der PTBS, der BPS und eine hohe Dissoziationsneigung vereint und in die bisherigen Klassifikationssysteme nur ungenügend eingeordnet werden kann. Die Konzeptualisierung dieser komplexen Traumafolgestörungen ist derweil im Fokus intensiver Diskussionen - Kapitel 1.1.4 soll hierzu einen Einblick ermöglichen.

\subsubsection{Die Posttraumatische Belastungsstörung}

Der Begriff der Posttraumatischen Belastungsstörung (PTBS) beschreibt eine spezifische Symptomkombination, die protrahiert nach traumatischen Belastungen auftreten kann (Schellong 2013, Flatten et al. 2013). Die Symptome der PTBS können in drei Hauptgruppen unterteilt werden (Maercker 2013, Flatten et al. 2013):

- Intrusionen: Das unkontrollierte Wiedererleben des Traumas z. B. in Form von Flashbacks, Albträumen und Bildern. Die Erinnerungen, Wahrnehmungen und Gedanken 
sind meist aufdrängend und belastend, ein Ausblenden ist in der Regel nicht möglich. Nicht selten werden sie von einem traumaassoziierten Reiz ,getriggert“.

- Vermeidung/Numbing: Traumaassoziierte Stimuli („Trigger") werden gemieden, und es kommt zu einer emotionalen Taubheit („Numbing ${ }^{\circ}$ ). Teilnahmslosigkeit und Interessenverlust, dissoziative Zustände sowie die partielle Amnesie sind weitere Beispiele für diesen Symptomkomplex.

- Hyperarousal: Eine vegetative Übererregung, die sich beispielsweise in Form von Schlafstörungen, vermehrter Schreckhaftigkeit und Reizbarkeit, Konzentrationsstörungen sowie Affektintoleranz äußert.

Die Diagnose Posttraumatische Belastungsstörung wird anhand des Klassifikationssystems ICD-10 gestellt, wenn innerhalb von sechs Monaten nach einem traumatischen Erlebnis intrusive Symptome, Vermeidungsverhalten (gegenüber traumaassoziierten Stimuli) und entweder Erinnerungslücken bezüglich des Traumageschehens oder Hyperarousal-Symptome auftreten (Maercker 2013). Dabei ist die Diagnose posttraumatic stress disorder des amerikanischen DSM-IV und -V (Diagnostic and Statistical Manual of Mental Disorders) weitgehend mit der Klassifizierung der ICD-10 vergleichbar. Während das DSM IV jedoch noch drei Symptomgruppen (s. o. Intrusionen, Vermeidung/Numbing und Hyperarousal) unterscheidet, ist eine der Neuerungen des DSM-V die Aufteilung des Symptomkomplexes avoidance/numbing in die beiden neuen Symptomkomplexe avoidance und persistent negative alterations in cognitions and mood (Tye et al. 2015). Letzteres umfasst nun die ehemals unter dem Stichwort Numbing aufgeführten Symptome. Aus konzeptioneller Sicht ist diese Aufteilung insofern sinnvoll, als Vermeidungssymptome eher phobischen Charakter besitzen, während Numbing-Symptome verschiedene Merkmale negativer Affektivität zusammenfassen (Maercker 2013).

\subsubsection{Dissoziative Störungen}

In der Psychologie besteht nach wie vor keine Einigkeit über die genaue Definition des Phänomens Dissoziation (Mattheß und Schüepp 2013). In der ICD-10 werden die dissoziativen Störungen und die Konversionsstörungen synonym genutzt und als „der teilweise oder völlige Verlust der normalen Integration von Erinnerungen an die Vergangenheit, des Identitätsbewusstseins, der unmittelbaren Empfindungen, sowie der Kontrolle von Körperbewegungen" beschrieben (WHO 2014, S. 212). Die ICD-10 fasst somit sowohl Symptome auf der psychischen Ebene als auch Symptome auf der körperlichen Ebene als dissoziative Störungen zusammen. Eine Unterscheidung dieser beiden Ebenen wird jedoch weiterhin als sinnvoll angesehen (Fiedler 2001).

Demnach können die dissoziativen Störungsbilder folgendermaßen auf die beiden Gruppen aufgeteilt werden:

- Dissoziative Bewusstseinsstörung (Dissoziation auf rein psychischer Ebene): Dissoziative Amnesie, dissoziative Fugue, dissoziativer Stupor, Trance- und Besessenheits- 
zustände, Ganser-Syndrom, dissoziative Identitätsstörung sowie dissoziative Störungen (gemischt)

- Konversionsstörungen oder auch pseudoneurologische dissoziative Störungen (Dissoziation auf der körperlichen Ebene): Dissoziative Bewegungsstörungen, dissoziative Krampfanfälle, dissoziative Empfindungs- und Sensibilitätsstörungen

Im Gegensatz zu der ICD-10 definieren das DSM-IV und das DSM-V dissoziative Störungen als rein psychische Phänomene und ordnen die Konversionssymptome bei den somatic disorders bzw. bei den somatic symptom and related disorders ein (American Psychiatric Association 1994, 2013).

Zwischen Trauma und Dissoziation besteht ein enger Zusammenhang. Vor allem Zustände der Depersonalisation und Derealisation sowie Amnesien finden sich als dissoziative Langzeitfolgen bei Trauma-Opfern. Auch die PTBS ist mit dissoziativen Phänomenen assoziiert. Regelmäßig erhöhte Werte auf der Dissociative Experience Scale (DES, s. Kap. 3.2.1.4) bestätigen diese Beobachtung (Flatten et al. 2004, Fiedler 2001). Erklärungskonzepte gehen davon aus, dass es im Zuge der Traumatisierung zu einer Abspaltung der für das Individuum zu bedrohlichen Emotionen, Kognitionen oder auch motorischen und sensorischen Sinneseindrücke kommt (Freyberger und Spitzer 2005). Die dissoziative Identitätsstörung zeigt sich als die extremste Form dieser Separation, indem sie Assoziationen der Identität betrifft. Es entwickeln sich zwei verschiedene Zustände des Selbst: ein „alltagstaugliches“ und ein „traumatisiertes Ich“. Die Dissoziation stellt einen Schutzmechanismus bzw. eine Bewältigungsstrategie bezüglich der Traumatisierung dar, wirkt sich jedoch im späteren Leben oft negativ aus, indem sie beispielsweise mit einem gestörten Selbsterleben und einer kognitiven Leistungsminderung einhergeht (Flatten et al. 2004).

\subsubsection{Die Borderline-Persönlichkeitsstörung}

Die Borderline-Persönlichkeitsstörung (BPS) ist laut DSM-V durch „ein tiefgreifendes Muster von Instabilität in zwischenmenschlichen Beziehungen, im Selbstbild und in den Affekten sowie von deutlicher Impulsivität" gekennzeichnet (American Psychiatric Association 2015, S. 908). Die Diagnosekriterien des DSM-IV bzw. -V für dieses Störungsbild umfassen folgende Punkte: „Impulsivität in mindestens zwei potenziell selbstschädigenden Bereichen; wiederholte suizidale Handlungen, Suiziddrohungen; affektive Instabilität und Stimmungsschwankungen; chronisches Gefühl von Leere; unangemessene heftige Wut; ausgeprägte Instabilität des Selbstbildes oder der Selbstwahrnehmung; vorübergehende paranoide und dissoziative Symptome; Muster instabiler, aber intensiver zwischenmenschlicher Beziehungen (Idealisierung und Entwertung); verzweifeltes Bemühen, Verlassenwerden zu vermeiden“ (Sack et al. 2011, S. 200). Hierbei müssen fünf dieser neun Kriterien für die Diagnose Borderline-Persönlichkeitsstörung erfüllt sein. 
In der ICD-10 werden bei der emotional instabilen Persönlichkeitsstörung ein impulsiver Typus sowie ein Borderline-Typus unterschieden, die beide durch „Impulsivität und mangelnde Selbstkontrolle“ charakterisiert sind. Bei dem Borderline-Typus sind zusätzlich „oft das eigene Selbstbild, Ziele und ,innere Präferenzen“ (einschließlich der sexuellen) unklar und gestört“. Es besteht zudem meist „ein chronisches Gefühl innerer Leere“. Auch eine „Neigung zu intensiven, aber unbeständigen Beziehungen“, die zu „wiederholten emotionalen Krisen“ u. a. auch mit „Suiziddrohungen oder selbstschädigenden Handlungen“ führen kann, wird vom ICD-10 als Kennzeichen des Borderline-Typus beschrieben (WHO 2014, S. 280). Damit entspricht der Borderline-Typus der emotional instabilen Persönlichkeitsstörung weitgehend der DSM-V-Definition der BPS.

Bei Patienten mit einer BPS finden sich gehäuft kindliche Traumatisierungen in ihrer Vergangenheit, weshalb eine Traumaätiologie dieser Persönlichkeitsstörung diskutiert wird. Zanarini et al. befragten 358 Patienten mit einer Borderline-Persönlichkeitsstörung zu traumatischen Erfahrungen in ihrer Kindheit (Zanarini et al. 1997). Es gaben 91,3 \% eine emotionale, verbale, physische oder sexuelle Misshandlung an, 92,2\% schilderten Vernachlässigung. 61,5\% der BPS-Patienten erlitten sexuellen Missbrauch in ihrer Kindheit. Dies stimmt mit Zanarinis später geäußerter Einschätzung überein, dass bei der Borderline-Persönlichkeitsstörung etwa 40-70 \% der Betroffenen sexuellen Missbrauch vor ihrem 18. Geburtstag erlebt haben (Zanarini 2000). Yen et al. (2002) wiesen nach, dass Patienten mit einer BPS im Vergleich zu anderen Persönlichkeitsstörungen häufiger von traumatischen Ereignissen (insbesondere sexueller Missbrauch in der Kindheit) berichten, öfter die Diagnosekriterien der PTBS erfüllen sowie jünger bei ihrer ersten Traumatisierung waren. Zanarini et al. (2002) zeigten zudem, dass ein Zusammenhang zwischen der Schwere des sexuellen Missbrauchs in der Kindheit und der Symptomlast bzw. der Schwere der BPS besteht. Auch das Ausmaß anderer Formen des Missbrauchs sowie einer Vernachlässigung in der Kindheit scheinen bei der Ausprägung der BPS einen Einfluss zu nehmen (Zanarini et al. 2002).

Trotz dieser Zahlen wird die Traumaätiologie der BPS weiterhin in Frage gestellt. Von verschiedener Seite wird darauf hingewiesen, dass eine Assoziation zwischen kindlichem Trauma und BPS zwar bestehe, das Trauma jedoch weder einen notwendigen noch einen ausreichenden Faktor für die Entwicklung einer BPS darstelle (Sack et al. 2011, Paris 2011, Lieb et al. 2004).

Für Sack et al. (2011) ist die Frage der traumatischen Genese der BPS auch eine Frage der Traumadefinition. Indem die Klassifikationssysteme DSM-IV und ICD-10 eine sehr eng gefasste Definition für einer PTBS zugrundeliegende Traumatisierungen verwenden, werden viele Entwicklungstraumatisierungen, wie die wiederholte psychische, physische und sexuelle Gewalt an Kindern, nicht explizit erfasst. Gerade für diese Formen der Traumatisierungen finden sich jedoch, wie oben beschrieben, bei BPS-Patienten besonders hohe Prävalenzzahlen (Sack et al. 2011). 
Auch aufgrund neuer Erkenntnisse auf genetischer und biologischer Ebene wird zunehmend von einigen Autoren ein multifaktorielles Modell zur Entstehung der BPS präferiert. Biologische und traumatische sowie weitere bislang nicht identifizierte Faktoren wären demnach gemeinsam an der Genese einer Borderline-Störung beteiligt (Gabbard 2005, Leichsenring et al. 2011). So untersuchten Ball und Links anhand der Literatur aus den Jahren 1995 bis 2007, ob die kindliche Traumatisierung als kausal für die BPS angesehen werden kann. Die Autoren kamen zu dem Schluss, dass das Kindheitstrauma am ehesten einen ätiologischen Faktor in einem solchen multifaktoriellen Modell zu den Ursachen der BPS darstellen könnte (Ball und Links 2009).

In Kapitel 1.1.4 wird deutlich, dass die Frage nach der Assoziation von BPS und Trauma auch für aktuelle Diskussionen zur Klassifikation komplexer Traumafolgestörungen entscheidend ist. Für das dort erläuterte Konzept des externalisierenden und internalisierenden Typus einer PTBS ist eine traumatische Genese der BPS, zumindest in einem multifaktoriellen Modell, Voraussetzung.

\subsubsection{Komplexe Traumafolgestörungen}

In der klinischen Praxis zeigt sich, dass die sehr eng gefassten diagnostischen Kriterien der ICD-10 und des DSM IV bzw. V nur unzureichend die Bandbreite an Symptomen, die mit einer PTBS einhergehen können, abbilden. Insbesondere Patienten mit schweren kindlichen und oft auch mehrfachen Traumatisierungen leiden unter einem deutlich vielfältigeren und komplexeren Erkrankungsbild. Ihr Verlauf ist in der Regel schwer und eine Therapie geht mit geringeren Erfolgsaussichten einher als es bei einer „einfachen PTBS“ der Fall ist.

Hieran wird deutlich, dass die Posttraumatische Belastungsstörung keinesfalls - wie früher lange angenommen - ein einheitliches klinisches Bild infolge ganz unterschiedlicher Arten der Traumatisierungen darstellt. Damals wurde sie häufig als eine gemeinsame Endstrecke für die verschiedenen Traumaformen angesehen (Maercker 2003b). Inzwischen hat sich jedoch der Vorschlag von Lenore Terr durchgesetzt, nach dem Traumata in Typ 1 und Typ 2 eingeteilt werden (Terr 1991). Ein Typ 1-Trauma entspricht einem einmaligen Ereignis, während ein Typ 2-Trauma mehrfache und/oder lang andauernde Traumatisierungen beschreibt, die meist durch eine besondere Schwere gekennzeichnet sind. PTBS-Patienten mit einem Typ 2-Trauma zeigen dementsprechend oft schwierigere und langwierigere Verläufe, sodass dies bei ihrer Therapie entsprechend berücksichtigt werden sollte. Zudem scheint auch die Ursache des Traumas von Bedeutung zu sein. Es zeigen sich Unterschiede zwischen den Folgen des sog. man made disaster, also der durch andere Menschen aktiv verursachten Traumatisierung, und den Auswirkungen von sog. Akzidentellen Traumatisierungen wie Naturkatastrophen, Unfällen und ähnlichen Katastrophen (Schellong 2013). So ist auch die Prävalenz einer PTBS von der Traumaursache abhängig. Nach Verkehrsunfällen liegt sie bei ca. 10 \%, während nach Vergewaltigungen bei etwa der Hälfte der Betroffenen eine PTBS auftritt (Flatten et al. 2013). 
Für eine differenzierte Betrachtung des Störungsbilds wird neben der Unterscheidung verschiedener Traumatypen auch eine Konzeptualisierung der verschiedenen Symptomausprägungen der PTBS angestrebt. Das hierfür von Herman entwickelte Konzept der komplexen Posttraumatischen Belastungsstörung (kPTBS) dient der Beschreibung des charakteristischen syndromalen Störungsbilds, das sich besonders häufig nach chronischen Typ 2Traumatisierungen findet (Herman 1993, Herman 1992). Die kPTBS umfasst dafür die Symptombereiche Störungen der Affektregulation, dissoziative Symptome, eine gestörte Selbstwahrnehmung, Störungen der Sexualität und Beziehungsgestaltung, somatoforme Körperbeschwerden und Veränderungen persönlicher Glaubens- und Wertvorstellungen. Das Konzept der kPTBS wurde unter der Bezeichnung DESNOS (disorders of extreme stress not otherwise specified) in das DSM-IV aufgenommen - allerdings nicht als diagnostische Kategorie, sondern als zusätzliches klinisches Beschreibungsmerkmal der PTBS für Forschungszwecke. Im amerikanischen Sprachraum werden seitdem die Begriffe komplexe PTBS und DESNOS synonym verwendet (Sack 2004a, Sack et al. 2011). Die viel diskutierte Aufnahme von DESNOS als eigenständige Diagnose in das DSM-V erfolgte jedoch nicht (Friedman 2013). Die Autoren des DSM-V sahen es nicht als erwiesen an, dass DESNOS nicht nur eine besonders schwere Form der PTBS, sondern eine eigenständige Diagnose darstelle. Diese Zweifel waren u. a. auch darin begründet, dass ein Großteil der Patienten mit DESNOS gleichzeitig auch die diagnostischen Kriterien für eine PTBS erfüllt (Resick et al. 2012, Kapfhammer 2014).

In der ICD-10 findet sich die Diagnose ,andauernde Persönlichkeitsänderung nach Extrembelastung“, die Hermans kPTBS sehr ähnlich ist (WHO 2014, S. 286; Zehl et al. 2013). Allerdings bezieht sich diese Diagnose nur auf Traumatisierungen im Erwachsenenalter. Die häufigen Traumafolgestörungen nach kindlichen Traumatisierungen werden nicht erfasst. So wundert es kaum, dass sich diese Kategorie bislang nicht in der klinischen Praxis durchsetzen konnte (Sack 2004a).

Sack et al. (2011) verglichen die DSM-IV-Diagnosekriterien der Borderline-Persönlichkeitsstörung mit den Kriterien der DESNOS im DSM-IV. Sieben der neun Kriterien für die BPS finden sich auch bei den DESNOS, nämlich die Impulsivität mit risikoreichem/selbstschädigendem Verhalten, das suizidale Verhalten, die Störung/Instabilität der Affekte, die Wut, die Störungen des Selbstbildes, die Dissoziation und die Störung/Instabilität der Beziehungen (vgl. auch S. 3, Sack et. al. 2011). Die somatoforme Symptomatik sowie die Perspektivlosigkeit bzw. der Verlust von Überzeugungen sind gleichzeitig die einzigen beiden Kriterien von DESNOS, die nicht auch für die BPS gelten (Sack et al. 2011). Es wird deutlich, dass es einen hohen Überschneidungsbereich der beiden Störungsbilder geben muss. Sack et al. (2013) konnten nachweisen, dass $55 \%$ der Patienten mit einer Borderline-Diagnose gleichzeitig die Kriterien der komplexen Posttraumatischen Belastungsstörung erfüllten. Außerdem zeigten 41 \% der BPS-Patienten eine komplexe dissoziative Störung, die sich in Form von Alltagsamnesien, Stimmenhören und Identitätsstörungen äußerte (Sack et al. 
2013). Zehl et al. sehen in dissoziativen Phänomenen einen „äußerst charakteristischen Symptombereich" der kPTBS (Zehl et al. 2013, S. 430).

Diese weitreichenden Überschneidungen der BPS und der kPTBS lassen eine gemeinsame Konzeptionalisierung der beiden Störungsbilder sinnvoll erscheinen. So können BPS und kPTBS als „Ausprägungen einer gemeinsamen, durch Entwicklungstraumatisierungen ausgelösten Grundproblematik“ aufgefasst werden (Sack et al. 2011, S. 201). Hinweise hierauf lieferten Miller und Resick, als sie mithilfe einer Clusteranalyse bei 143 Vergewaltigungsopfern einen externalisierenden, einen internalisierenden und einen einfachen Typ der Posttraumatischen Belastungsstörung differenzieren konnten (Miller und Resick 2007, Zehl et al. 2013). Der externalisierende Typus ist durch impulsives und aggressives Verhalten charakterisiert, auf Störungsebene sind besonders die Cluster-B-Persönlichkeitsstörungen und der Substanzmissbrauch assoziiert. Zu den Cluster-B-Persönlichkeitsstörungen zählen die BPS sowie die histrionische, die antisoziale und die narzisstische Persönlichkeitsstörung. Symptome wie depressive Reaktionen, selbstverletzendes Verhalten, dissoziative Phänomene, ängstliches Vermeidungsverhalten sowie der soziale Rückzug sind dagegen den Patienten des internalisierenden Typus zuzuordnen (Sack et al. 2011). Depressionen und schizoide sowie ängstlich-vermeidende Persönlichkeitsstörungen werden hier besonders häufig diagnostiziert. Außerdem findet sich eine höhere Symptomlast als bei dem externalisierenden Typus (Zehl et al. 2013). Der einfache Typus zeigt die klassische Symptomtrias von Intrusionen, Vermeidungsverhalten und Hyperarousal und ist - im Gegensatz zum externalisierenden und internalisierenden Typus - nicht durch ein besonders negatives Temperament gekennzeichnet. Auch Achse-1- Komorbiditäten wie Depressionen sind seltener mit der einfachen PTBS vergesellschaftet (Schellong 2013).

Laut Sack et al. kann nach diesem Konzept die Borderline-Persönlichkeitsstörung als externalisierender Typus einer komplexen Traumafolgestörung gewertet werden, während Hermans komplexe PTBS den internalisierenden Typus darstellt. Klinische Beobachtungen führen zu der Vermutung, dass es auch Mischformen geben muss, denn beim gleichen $\mathrm{Pa}-$ tienten kann sich die internalisierende mit der externalisierenden Symptomatik abwechseln. Typischerweise sind diese Mischformen bei Patienten zu entdecken, die auch eine starke dissoziative Symptomatik aufweisen (Sack et al. 2011). Die beschriebene Subtypisierung der komplexen Traumafolgestörung nach Externalisierungs- und Internalisierungskriterien deckt sich mit den Ergebnissen zahlreicher Studien (u. a. Miller et al. 2003, Miller et al. 2004, Sellbom und Bagby 2009, Forbes et al. 2010, Klosterman Rielage et al. 2010, Zehl et al. 2013, Castillo et al. 2014, Carleton et al. 2015).

Die Diskussionen bezüglich der Konzeptionalisierungen und Kategorisierung der PTBS, kPTBS und damit auch assoziiert der BPS haben bisher zu keinem Konsens geführt, daher wird auch in Zukunft intensiv in diesem Gebiet geforscht werden müssen. Dies zeigt jedoch auch die Komplexität sowie die Notwendigkeit einer differenzierten Betrachtung dieser Störungsbilder. 


\subsection{Physiologische Stressreaktionen}

Im angloamerikanischen Raum wird die PTBS mit dem Begriff posttraumatic stress disorder (PTSD) benannt - eine Bezeichnung, die sehr viel treffender für eine Erkrankung erscheint, die so eng mit Stress und seinen Auswirkungen verknüpft ist (Sachsse 2004). Dabei ist der Stress nicht nur hinsichtlich seiner ätiologischen und pathophysiologischen Aspekte entscheidend, sondern er prägt auch das symptomatische Bild der PTBS, wie die HyperarousalSymptomatik zeigt. Folglich ist für ein besseres Verständnis der PTBS eine möglichst genaue Kenntnis der Stressphysiologie und der verschiedenen Stresssysteme unabdingbar.

Stressreaktionen dienen der Anpassung des Körpers an eine potentiell bedrohliche Situation und haben sich evolutionär als erfolgreiche Strategien erwiesen. Laut Panksepp (1998) kann das menschliche Gehirn bei einer möglichen Bedrohung entweder auf das Paniksystem oder aber auf das Furchtsystem zurückgreifen. In der Tierwelt findet sich zudem das Erstarrungssystem, das aufgrund seiner Parallelen zur Dissoziation von Interesse ist.

\subsubsection{Paniksystem}

Sind Säuglinge oder kleine Kinder von ihren Bezugspersonen, meist der Mutter, getrennt, empfinden sie dies als substanzielle Bedrohung. Ihr Paniksystem wird aktiv und durch Weinen und Schreien versuchen sie ihre Mutter auf ihre Situation aufmerksam zu machen. Diese als distress vocalizations bezeichneten Laute gibt es auch in der Tierwelt: Jungtiere rufen mit Jaulen, Piepsen und ähnlichem nach ihrer Mutter. Die distress vocalizations lösen in der Mutter eine Stressreaktion aus; der Stress lässt sich nur beenden, wenn die Mutter ihrem Kind hilft. Die beidseitige Lösung des Stresses bei Kind und Mutter zeigt einen positiven Effekt auf die Bindung zwischen den beiden. Das Paniksystem wird daher auch Bindungssystem genannt. Neurobiologisch zeigen sich folgende Korrelate für die distress vocalizations: das periaquäduktale Grau in der Formatio reticularis, das laterale Septum im limbischen System und der Gyrus cinguli. Eine Erregung des Paniksystems wird durch den Neurotransmitter Glutamat erreicht, während Opioide und Oxytocin beruhigend wirken. Das Paniksystem ist hierbei eng mit dem Parasympathikus assoziiert und es treten in Situationen der Panik vegetative Symptome wie ein Kloß im Hals, Druck in der Brust oder aber weiche Knie auf (Hüther und Sachsse 2007).

Das Bindungssystem spielt laut Panksepp nicht nur in der frühkindlichen Mutter-KindBindung eine entscheidende Rolle, sondern ist auch bei der Herden- bzw. Gemeinschaftsbildung von Bedeutung. So wird die soziale Überlebensstrategie der Herdenbildung über die gleichen Zentren und Stoffe vermittelt, die auch bei der Mutter-Kind-Bindung zum Schutz des Säuglings führen. Da viele Zentren des Gehirns hochspezialisiert arbeiten, ist diese Überschneidung sehr bemerkenswert (Panksepp 1998, Sachsse 2003). 


\subsubsection{Furchtsystem}

Fight or flight - in der Evolution der Menschheit haben sich diese beiden Lösungsstrategien bei Gefahrensituationen bewährt. Seit Cannons Veröffentlichungen (1914) wird allgemein davon ausgegangen, dass das Furchtsystem den Körper auf ebendiese Handlungen vorbereitet, auf eine Flucht oder einen Kampf. Das Furchtsystem wird durch Stress aktiviert und vermittelt über Adrenalin und Noradrenalin eine Sympathikusreaktion. Neurobiologisch beteiligen sich der Locus caeruleus der Formatio reticularis, das periaquäduktale Grau sowie die Amygdala und der Hippocampus des limbischen Systems am Furchtsystem. Der Körper befindet sich als Reaktion auf den Stress in einer Art Alarmbereitschaft und sucht nach einer Möglichkeit, den Stress zu beenden. Gelingt dies, wird das Furchtsystem durch eine Ausschüttung von Dopamin und Opiaten beruhigt und die Lösungsstrategie wird als erfolgreich abgespeichert. Dopamin wirkt neuroplastisch und kann somit Genexpressionen verändern und Synapsen neubilden, sodass die Verschaltungen des Gehirns, die für die Lösung des Problems wichtig waren, gestärkt werden. Das Furchtsystem ist somit die Grundlage für das kognitive Lernen. Diese durch das Furchtsystem vermittelten Lernprozesse gelten jedoch auch für „Lösungsstrategien“ wie Alkoholabusus, Bulimie und Zwangsrituale, die in der akuten Situation ebenfalls eine Stressreduktion bewirken (Hüther und Sachsse 2007, Hüther 1996).

Bei zu langem oder zu heftigem Stress wird jedoch die corticotrope Stressachse aktiviert, die über die HPA-Achse Hypothalamus - Hypophyse (pituitary) - Nebennierenrinde (adrenal glands) definiert ist. Der Hypothalamus sezerniert das CRH (corticotropin-releasing hormone), das über die Hypophyse die Produktion von ACTH (Adrenocorticotropes Hormon) stimuliert. Bei der ACTH-Bildung entsteht als Nebenprodukt $\beta$-Endorphin, das wiederum das Paniksystem beruhigt. ACTH führt an der Nebennierenrinde zur Cortisol-Bildung. Cortisol wirkt über einen negativen Feedbackmechanismus dem Furchtsystem entgegen und dämpft dessen Effekte. Während geringe Cortisol-Dosen noch das Gedächtnis fördern, schwächen hohe Dosen das Erinnerungsvermögen (Yehuda 2001, Sachsse 2003, Ackermann et al. 2013).

Das Furcht- und das Paniksystem sind keine isoliert voneinander ablaufenden Systeme, vielmehr interagieren sie. Das Furchtsystem stellt hierbei einen wichtigen Beruhigungsmechanismus für das Paniksystem dar. Es bietet die Chance auf einen Ausweg aus der Situation, da es das Finden einer Lösungsstrategie ermöglicht. Das Paniksystem dagegen blockiert jede konstruktive Auseinandersetzung mit dem Problem, denn alles wird von einer lähmenden Panik eingenommen. Somit flüchten wir Menschen uns, wenn möglich, aus der Panik in die Furcht, mit der wir sehr viel leichter umzugehen vermögen (Hüther und Sachsse 2007). 


\subsubsection{Erstarrungssystem und Dissoziation}

In der Tierwelt ist die sog. ,freeze-Reaktion“, auch als „Totstellreflex“ bekannt, häufig anzutreffen. Nach außen hin ist das Tier in einer Gefahrensituation erstarrt; es gibt weder Laute von sich noch zeigt es jegliche Regung. Innerlich befindet sich das Tier jedoch im Hyperarousal, einem Zustand höchster Erregung (Sachsse 2003). Bei Überlegenheit des Angreifers oder Unausweichlichkeit ist das freezing eine durchaus sinnvolle Überlebensstrategie. Es wird angenommen, dass es bei dieser Erstarrungsreaktion zu einer simultanen Erregung des Sympathikus und Parasympathikus kommt. Trotz hoher Adrenalinspiegel, findet sich beim freezing eine verminderte Herzfrequenz. Dies ist laut Sack (2004b) erklärbar durch den dominierenden Einfluss des Parasympathikus auf die Herzfrequenz. Außerdem werden endogene Opioide ausgeschüttet, welche eine Analgesie bedingen, die ebenfalls bei Erstarrungsreaktionen häufig beobachtet wurde.

Bei dem Menschen gibt es eine ähnliche Notfallreaktion in ausweglosen Situationen, bei denen weder Furcht- noch Paniksystem Hilfe versprechen: Die Dissoziation. Auch sie kann wie das freezing durch eine totale Bewegungsstarre gekennzeichnet sein. Daher wird der Totstellreflex der Tierwelt oft als Erklärungsmodell für die Dissoziation herangezogen. Die Dissoziation geht jedoch über die äußerliche Erstarrung des freezing hinaus. Die Patienten berichten, dass sie auch ,innerlich erstarrt“ sind und der Reizstrom nach innen unterbrochen zu sein scheint. Inwieweit dies auch in der Tierwelt existiert, ist schwierig zu erforschen (Sachsse 2003). Patienten mit starken dissoziativen Symptomen zeigen während des traumaassoziierten Stresses eine geringere Herzrate als Patienten mit geringen dissoziativen Symptomen. Auch hier ist also wie beim freezing eine deutlich andere autonome Reaktion als die klassische fight or flight-Antwort zu beobachten. Allerdings kann nach bisherigen Erkenntnissen die geringere Herzrate bei dissoziativen Stress am ehesten mit einem verminderten Arousal erklärt werden, denn im Gegensatz zum freezing ist bei dissoziativen Stress auch der Parasympathikustonus erniedrigt (Sack et al. 2012). So ist die freeze-Reaktion zwar in vielen Punkten ein hilfreiches Modell für die Dissoziation, es gibt jedoch auch deutliche Unterschiede zwischen diesen beiden Formen der Stressreaktion.

\subsection{Biologische Besonderheiten der PTBS}

In jüngerer Zeit wurden vielfältige körperliche Veränderungen bei der PTBS entdeckt. So wurden erhöhte Konzentrationen an zirkulierenden Entzündungszytokinen wie IL-6 festgestellt. Auf hormoneller Ebene wurden u. a. Veränderungen der Oxytocin-Regulation und der HPA-Achse beschrieben. Das autonome Nervensystem scheint Abweichungen bezüglich der Stressregulation aufzuweisen. Und auch auf neuroanatomischer Ebene konnten veränderte Gehirnstrukturen gefunden werden (De Bellis und Zisk 2014). Viele dieser Befunde werfen zurzeit noch mehr Fragen auf als sie beantworten können und doch liefern sie einen ersten Ausblick auf die umfassenden körperlichen Modifikationen, mit denen das Störungsbild, insbesondere in Bezug auf die Stressverarbeitung, verbunden ist. Besonders 
interessant ist dabei die Frage, ob es sich bei den biologischen Veränderungen um die Folge eines Traumas, also beispielsweise um eine symptomatische maladaptive Stressbewältigung, oder aber um einen ursächlichen, prädiktiven oder verstärkenden Faktor bei der Entstehung der Erkrankung handelt. Die folgenden Ausführungen konzentrieren sich auf einige zentrale somatische Besonderheiten der PTBS bei Erwachsenen.

\subsubsection{Dysregulation der HPA-Achse}

Aufgrund der ausgeprägten Hyperarousal-Symptomatik bei der PTBS liegt es nahe, einen Hypercortisolismus als Zeichen eines chronischen Stresssyndroms zu vermuten. Tatsächlich konnten Abweichungen in der HPA-Achse bei der PTBS nachgewiesen werden. Auch wenn die Datenlage nicht ganz einheitlich ist und vereinzelt auch erhöhte Cortisolspiegel gemessen werden konnten: Die Mehrheit der Studien fand überraschenderweise ein erniedrigtes oder zumindest nicht gegenüber Gesunden erhöhtes Cortisollevel bei der PTBS (Pervanidou 2008). Gleichzeitig wurde jedoch im Liquor von PTBS-Patienten eine erhöhte basale CRHKonzentration nachgewiesen (Bremner et al. 1997, Baker et al. 1999, Sautter et al. 2003). Diese Kombination ist ungewöhnlich, denn während das erhöhte CRH-Level auf eine suprahypophysäre Aktivierung hindeutet und eine verminderte Aktivität der HPA-Achse eher ausschließt, bleibt eine gesteigerte Cortisolreaktion aus. Dies wird von Yehuda auch als „HPA-Paradoxon“ betitelt (Yehuda 2001, S. 56). Gegen eine allgemeine Hypoaktivität der HPA-Achse spricht zudem, dass die Konfrontation von PTBS-Patienten mit traumaassoziierten Erinnerungen zu einem starken Anstieg des Cortisolspiegels führt (Elzinga et al. 2003). In mehreren Studien wurde mit einem Dexamethason-Test eine verstärkte CortisolSuppression ausgelöst, welche auf eine gesteigerte negative Rückkopplung hinweist (Stein et al. 1997, Yehuda et al. 2002, Newport et al. 2004). Die gesteigerte negative Rückkopplung könnte durch eine erhöhte Sensitivität der Glukokortikoid-Rezeptoren (GR) zustande kommen, die ebenfalls bei der PTBS gefunden wurde (Yehuda et al. 2004). Es wird vermutet, dass diese erhöhte Sensitivität eine zentrale Bedeutung für all die beschriebenen Veränderungen der HPA-Achse einnimmt. Interessanterweise wurde bei akutem Stress sowie bei Depressionen ein sehr gegensätzliches Verhalten der Stressachse mit einem gleichzeitig erhöhten CRH- und Cortisol-Level sowie mit einer verminderten Suppression im Dexamethason-Test und einer verringerten Sensitivität der GR beobachtet (Yehuda 2009).

\subsubsection{Neuroanatomische Veränderungen}

Der Fund von reduzierten Hippocampi-Volumina bei PTBS-Patienten löste umfassende Forschungen auf diesem Gebiet aus. Der Hippocampus ist nicht nur an der Stressadaption beteiligt (s. Kap. 1.2.2), sondern spielt u. a. auch eine Schlüsselrolle bei Gedächtnis- und Lernvorgängen. In zwei Metaanalysen bestätigen sowohl Karl et al. (2006) als auch Woon et. al. (2010), dass das Hippocampus-Volumen bei erwachsenen Personen mit PTBS verglichen zu Kontrollgruppen mit und ohne Traumaexposition signifikant kleiner ist. Aber auch die 
traumaexponierte Gruppe ohne PTBS wies ein verringertes Hippocampus-Volumen gegenüber Probanden ohne Traumaexposition auf. Karl et al. (2006) beschrieben darüber hinaus, dass die Effektstärke für die Assoziation zwischen geringen Hippocampusvolumina und der PTBS mit steigender PTBS-Schwere zunimmt. Außerdem fanden sie ein signifikant verringertes Volumen der linken Amygdala im Vergleich zu den beiden Kontrollgruppen sowie eine signifikante Verkleinerung des anterioren cingulären Cortex gegenüber den Traumaexponierten ohne PTBS. Kinder mit PTBS zeigten in den analysierten Studien andere strukturelle Veränderungen und keine Hippocampusreduktion.

Pathophysiologie und Zeitpunkt der Hippocampusreduktion bleiben bislang unklar. Eine Hypothese sieht, unterstützt von Zwillingsstudien (Gilbertson et al. 2002, Gurvits et al. 2006), das geringe Hippocampus-Volumen als einen Prädiktor für die Entwicklung einer PTBS. Andere vermuten die Ursache in einem neurotoxischen Effekt des Traumas, beispielsweise in Form eines Hypercortisolismus, denn es ist bekannt, dass dieser mit einer Reduktion des Hippocampusvolumens einhergeht (McEwen 2007). Da jedoch PTBS-Patienten allgemein keinen erhöhten Cortisolspiegel aufzuweisen scheinen (s. Kap. 1.3.1), bleibt auch dieses Erklärungsmodell strittig (Woon et al. 2010).

\subsubsection{Dysregulation des autonomen Nervensystems}

Hinter der Hyperarousal-Symptomatik verbirgt sich in erster Linie eine autonome Dysregulation. Hierauf deuten zum einen Studien hin, die erhöhte Catecholaminkonzentrationen im Urin nachwiesen (Kosten et al. 1987, Hawk et al. 2000). Gleichzeitig konnte ein erhöhter Noradrenalin-Spiegel in Plasma und Liquor gemessen werden (Geracioti et al. 2001, Yehuda et al. 1998). Diese sowohl peripher als auch zentral gesteigerte noradrenerge Aktivität kann als verstärkte sympathische Aktivität gewertet werden - ein Zeichen für pathologische Veränderungen im autonomen Nervensystem (Pervanidou 2008).

Zum anderen bieten die verschiedenen kardiovaskulären Parameter eine gute Möglichkeit, das autonome Nervensystem (ANS) zu beurteilen. Eine Vielzahl an Studien wurde bereits zur Psychophysiologie der PTBS durchgeführt, Pole fasste einige davon 2007 in einer Metaanalyse mit über 1000 Probanden zusammen (Pole 2007). Hiernach geht die PTBS mit einem erhöhten Arousal in Ruhe sowie mit einer verstärkten autonomen Reaktivität bei schreckund traumaassoziierten Reizen einher. Dies äußert sich vor allem in einer erhöhten Herzrate (HR), die bei PTBS-Probanden im Vergleich zu Probanden ohne PTBS in Ruhe, bei Schreckreaktionen oder bei traumaassoziierten Stressoren gemessen wurde. Die erhöhte Ruhe-HR wurde bereits in einer vorausgegangenen Metaanalyse beschrieben (Buckley und Kaloupek 2001) und findet sich auch in aktuelleren Studien zu männlichen Veteranen (Paulus et al. 2013) und Unfallopfern (Rabe et al. 2006). Tatsächlich sieht Pole in der Herzrate, über alle Studien hinweg betrachtet, einen sehr sensitiven und verlässlichen Parameter für die PTBS. Dies spiegelt sich auch in einer prospektiven Studie von Bryant et al. (2003) wider, in der eine erhöhte Herzrate im Monat direkt nach dem Trauma mit einer Sensitivität von 74 \% 
und einer Spezifität von 91 \% das Vorliegen einer PTBS zwei Jahre später vorhersagte. Da die oben beschriebenen erhöhten Noradrenalin-Spiegel primär zu einer Vasokonstriktion und damit zu einer Blutdrucksteigerung führen, wäre in Hinblick auf das sympathische System eine reaktive Reflex-Bradykardie zu erwarten. Die Herzrate steht jedoch sowohl unter sympathischen als auch parasympathischen Einfluss, sodass der Fund einer erhöhten Herzrate auf ein vagales Defizit hindeutet. Übereinstimmend hiermit ist zwar auch die hauptsächlich sympathische Messgröße Hautleitfähigkeit laut Pole (2007) in Ruhe erhöht, jedoch weniger stark als die Herzrate. Die Diskrepanz der beiden Parameter kann wiederum durch eine verminderte vagale Aktivität erklärt werden. Die Ruhe-HR wäre demnach weniger aufgrund einer basalen Sympathikus-Hyperaktivität, sondern vielmehr aufgrund einer basalen Parasympathikus-Hypoaktivität erhöht. Der sympathische Einfluss auf die autonome Herzfrequenzkontrolle überwiegt - es besteht eine Dysbalance zwischen den beiden autonomen Gegenspielern (Sack et al. 2004). Systolischer und diastolischer Blutdruck sind in Ruhe ebenfalls, wenn auch moderat, bei der PTBS erhöht (Buckley und Kaloupek 2001, Pole 2007). Der diastolische Blutdruck erreicht jedoch eine höhere Effektstärke in Poles Metaanalyse und zeigt im Gegensatz zum systolischen Blutdruck auch bei traumaassoziierten Reizen erhöhte Werte im Vergleich zur Kontrollgruppe. Die PTBS scheint somit nicht nur insgesamt zu einem erhöhten Blutdruck zu führen, sondern wirkt insbesondere einem Druckabfall in den Gefäßen entgegen. Dies überrascht bei erhöhten Noradrenalin-Spiegeln und einer damit einhergehende Vasokonstriktion wenig. Eine der Limitationen der Metaanalyse ist laut Pole, dass sich disproportional viele der eingeschlossenen Studien auf männliche Veteranen fokussierten. Auch dass sich bezüglich der Effektgrößen der einzelnen Studien eine sehr große Heterogenität zeigte, sollte bei der Interpretation der Ergebnisse beachtet werden (Pole 2007).

Eine vagale Dysfunktion liefert das schlüssigste Erklärungskonzept für die von Pole beschriebenen Veränderungen des ANS bei der PTBS. Dies wird auch durch jüngste Erkenntnisse zur Herzratenvariabilität (HRV) gestützt, die Sammito et al. (2015) in einem Review analysieren. Hiernach ist die Herzratenvariabilität als Marker für die parasympathisch-sympathische Balance bei PTBS-Patienten verringert - ein Umstand, der für einen relativ verstärkten Sympathikuseinfluss spricht. Zur Bestimmung der Parasympathikus-Aktivität kann zudem die hochfrequente Komponente der HRV, die HF-HRV, isoliert betrachtet werden. Die Datenlage zur HF-HRV ist jedoch inkonsistent: Einige Studien stellten keinen Unterschied zwischen PTBS-Probanden und Kontrollgruppen fest, andere Studien beschreiben eine verringerte HF-HRV bei der PTBS, die als verringerter Parasympathikus-Tonus gedeutet werden könnte (Chang et al. 2013). Ein weiterer guter Marker für den Parasympathikus ist die Baroreflexsensitivität (BRS; s. Kap. 1.4.1), die bei einer vagalen Hypoaktivität vermindert sein müsste. Hughes et al. (2006) entdeckten eine Assoziation zwischen PTBS und niedriger BRS in Ruhe, allerdings nur bei Frauen, nicht bei Männern. Ob Moderatorvariablen diesen Geschlechterunterschied bedingen, konnte nicht abschließend geklärt werden (Hughes et al. 2006). Auch in einer weiteren Studie wiesen Frauen mit einer PTBS eine 
verringerte basale BRS auf, zudem zeigten sie während einer Ärgerreaktion ein im Vergleich zu einer Kontrollgruppe vermindertes Absinken der BRS (Hughes et al. 2007). Ähnliches hatten auch Cohen et al. (1998) festgestellt: die HF-HRV von PTBS-Probanden war bei ihren Messungen in Ruhe ebenfalls niedriger und sank bei traumaassoziiertem Stress im Vergleich zur Kontrollgruppe weniger stark ab. Die vagale Dysfunktion scheint somit auch eine parasympathische Hyporeagibilität einzuschließen.

Interessant ist in diesem Zusammenhang außerdem eine Studie, die bei jungen Erwachsenen mit PTBS eine höhere Wahrscheinlichkeit für eine orthostatische Hypotonie feststellte. Bei der orthostatischen Hypotonie kommt es zu einer inadäquaten sympathisch vermittelten Vasokonstriktion bei einem Lagerungswechsel. Im Sinne einer verringerten Disinhibition wird aber auch eine nur schwache Vagusantwort bei einem Lagerungswechsel als mögliche Genese der orthostatischen Hypotension genannt. Zu beachten ist der häufige Alkoholabusus der PTBS-Probanden in der Studie, der einen Einfluss auf die beschriebenen Ergebnisse gehabt haben könnte (Oddone et al. 2015).

Während bisher in der Regel die Stressreaktion des ANS im Fokus der Forschung stand, mehren sich die Hinweise, dass auch die autonome Erholung („Recovery ") nach einer Stressreaktion bei der PTBS gestört ist. Sack et al. (2004) entdeckten beispielsweise bei PTBSPatienten, die durch einen reduzierten Vagotonus im Sinne einer verminderten respiratorischen Sinusarrhythmie gekennzeichnet waren, eine verlängerte HR-Antwort auf traumaassoziierte Reize. Auch andere Studien fanden eine verringerte Rückbildungsfähigkeit nach Stress oder aber fehlerhafte Modulationen bei der Stressrückbildung. Insgesamt ist die Recovery jedoch noch vergleichsweise wenig erforscht, auch wenn vermutet wird, dass die Unfähigkeit zur typischen Recovery-Reaktion für die PTBS sehr charakteristisch sein könnte (Pole 2007). Albrecht und Bornschein, die den ersten Teil der hier vorliegenden Studie durchführten, konnten viele der soeben beschriebenen autonomen Besonderheiten der PTBS auch bei Patientinnen mit überwiegend komplexen Traumafolgestörungen nachweisen (Albrecht 2013, Bornschein 2014). Bornschein beschreibt im Vergleich zu einer Kontrollgruppe bei den PTBS-Patientinnen eine erhöhte Herzrate, einen erhöhten systolischen Blutdruck sowie eine verringerte BRS und HF-HRV in Ruhe. Gleichzeitig zeigte sich bei der PTBS auch eine allgemein geringere Stressreagibilität - nicht nur bei der BRS und HF-HRV wie schon bei Hughes et al. (2007) und Cohen et al. (1998), sondern auch bei der HR. Dies steht im Gegensatz zu Poles Metaanalyse (2007), die für die Herzrate eine eher verstärkte Reaktivität bei traumaassoziierten Stress- und Schreckreaktionen nahelegte. Albrecht (2013) und Bornschein (2014) verwendeten für ihre Messungen sowohl einen emotionalen als auch einen kognitiven, jedoch keinen direkt traumabezogenen Stressor. Der emotionale Stressor Babyschreien (Abspielen von Tonaufnahmen mit Babyschreien) ist noch vergleichsweise unerprobt und überraschte, indem er zu einer Parasympathikus-Reaktion bei gesunden Probandinnen führte und damit grundlegend andere Stressbewältigungsmechanismen ansprach als der kognitive Stressor Rechnen. Die vagale Stressreaktion während des Babyschreiens fiel 
bei den PTBS-Patientinnen trotz subjektivem Stressempfinden deutlich geringer aus, auch zu einer sympathischen Aktivierung kam es nicht. Dies stützt die These einer vorwiegend parasympathisch vermittelten Hyporeagibilität. Albrecht (2013) fand zudem Hinweise auf eine eingeschränkte Rückbildungsfähigkeit (Recovery) bei den Patientinnen.

Die aktuelle Studienlage legt biologische Modelle der PTBS nahe, die einer Dysfunktion des Parasympathikus mit einer vagalen Hyporeagibilität und einem verringerten vagalen Aktivitätsniveau bei der autonomen Dysregulation der PTBS eine entscheidende Rolle zuweisen und zu einer veränderten sympathovagalen Balance führen. Die reduzierte Antagonisierung des Sympathikus könnte dann auch das verstärkte Arousal in Ruhe und die erhöhte Reaktivität bei traumaassoziierten Stressoren erklären.

\subsection{Die sympathovagale Balance}

Als gutes Maß für die Funktionalität des autonomen Nervensystems gilt die sympathovagale Balance. Wie in Kapitel 1.3.3 beschrieben, ist eine sympathovagale Dysbalance, also ein Ungleichgewicht zwischen Sympathikus- und Parasympathikusaktivierung, kennzeichnend für die PTBS. Im Folgenden soll die Baroreflexsensitivität als eine mögliche Messgröße für die sympathovagale Balance vorgestellt werden. Außerdem soll die Assoziation zwischen PTBS und kardiovaskulären Erkrankungen in Hinblick auf die Rolle der autonomen Dysbalance betrachtet werden.

\subsubsection{Die Baroreflexsensitivität}

Neben der Herzratenvariabilität hat sich insbesondere die Baroreflexsensitivität (BRS) als Maß für die sympathovagale Balance etabliert. Der Barorezeptorreflex kontrolliert kurzfristige Blutdruckschwankungen und beeinflusst damit die Blutdruckvariabilität, nicht aber langfristige Trends des absoluten Blutdruckniveaus. Über arterielle Barorezeptoren in der Wand des Sinus caroticus und des Aortenbogens werden dem zentralen Nervensystem kontinuierlich Informationen zu den Veränderungen des Blutdrucks zugespielt. Eine Aktivierung der Barorezeptoren, die durch einen Anstieg des arteriellen Blutdrucks ausgelöst wird, beantwortet der Körper mit einer verstärkten Parasympathikus- und einer verminderten Sympathikusaktivierung. Indem beispielsweise Herzrate, Herzkontraktilität und peripherer Widerstand sinken, wird eine Blutdrucksenkung erreicht. Dagegen führt eine Deaktivierung der Barorezeptoren aufgrund eines sinkenden Blutdrucks zu der genau gegensätzlichen Reaktion des kardiovaskulären Systems: gesteigerte Sympathikus-, verminderte Parasympathikusaktivität und daraus resultierend Tachykardie, eine gesteigerte Herzkontraktilität sowie ein erhöhter peripherer Widerstand und damit ein Anstieg des Blutdrucks (La Rovere et al. 2008).

Wie empfindlich die Barorezeptoren auf Blutdruckschwankungen reagieren, kann an der Baroreflexsensitivität abgelesen werden. Hierfür werden Blutdruckschwankungen mit den korrespondierenden Veränderungen der Herzrate in Verhältnis gesetzt (s. Kap. 3.2.2). 
Verschiebt sich die sympathovagale Balance zu einer relativen Dominanz des Sympathikus, kommt es zu einer Verringerung der BRS. Umgekehrt steht eine erhöhte BRS für ein Überwiegen des Parasympathikuseinflusses (La Rovere et al. 1995).

\subsubsection{Auswirkungen einer Dysbalance}

Dank einer Reihe großangelegter Studien gibt es eine gute Evidenz dafür, dass die PTBS mit einem erhöhten Risiko für kardiovaskuläre Erkrankungen sowie mit einer erhöhten kardiovaskulären Mortalität einhergeht (z. B. Xue et al. 2012, Boscarino 2008, Kubzansky et al. 2009; Metaanalyse: z. B. Edmondson et al. 2013). Für diese Assoziation werden neben psychosozialen und verhaltensbezogenen Faktoren auch die biologischen Auffälligkeiten der PTBS, wie die autonome Dysbalance, als möglicher verbindender Mechanismus in Betracht gezogen (Edmondson und Cohen 2013). So finden sich bei Patienten mit einer Herzinsuffizienz ein erhöhter Sympathikus- sowie ein verringerter Vagotonus (Herrmann-Lingen 2011). Dementsprechend ist auch eine verminderte BRS messbar, die sich als prognostisch ungünstiger Faktor bei Herzinsuffizienz herausstellte (Pinna et al. 2005, La Rovere et al. 2009). Es wäre somit denkbar, dass die autonomen Veränderungen der PTBS die im Rahmen von kardiovaskulären Erkrankungen wie der Herzinsuffizienz auftretenden autonomen Störungen verstärken und so zu einer schlechteren Prognose beitragen (Herrmann-Lingen 2011). Hierauf deutet auch die Metaanalyse von Edmondson et al. (2012) hin, die eine Assoziation zwischen PTBS-Symptomen bei Patienten mit akutem Koronarsyndrom und einem erhöhten Risiko für das Wiederauftreten von kardialen Ereignissen sowie für eine erhöhte Mortalität beschreibt. Zudem konnte gezeigt werden, dass ein hoher vagaler Ruhetonus mit einer größeren Kapazität für Stressregulation, emotionalen Arousal und für Aufmerksamkeit verbunden ist (Sack et al. 2004). Die Hypoaktivität des Vagus bei der PTBS könnte folglich auch in Form einer maladaptiven Stressbewältigung zu einem erhöhten kardiovaskulären Risiko beitragen.

\subsection{Therapie der PTBS}

Das folgende Kapitel soll die speziellen Aspekte der Traumatherapie des Asklepios Fachklinikums Göttingen aufzeigen. Für einen Überblick zu den allgemeinen Prinzipien der PTBS-Therapie sei dagegen auf die Leitlinie von Flatten et al. (2013) verwiesen. Weiterhin sollen beispielhaft einige Studien zu der Frage nach einer Therapiewirkung auf die autonome Dysregulation der PTBS besprochen werden, wobei eine ausführliche Analyse in der Diskussion erfolgen wird. 


\subsubsection{Die Station 9 des Asklepios Fachklinikums Göttingen}

Die Station 9 des Asklepios Fachklinikums Göttingen ist spezialisiert auf die Behandlung von Patientinnen mit einer komplexen Posttraumatischen Belastungsstörung überwiegend nach Typ 2-Traumatisierungen. Als Komorbiditäten finden sich hierbei vorrangig die Borderline-Persönlichkeitsstörung und selbstverletzendes Verhalten, auch Depressionen und dissoziative Symptome sind häufig. Die Spezialstation bietet dabei Platz für die stationäre Therapie von jeweils 18 Patientinnen. Das von Sachsse entwickelte traumazentrierte Therapiekonzept integriert die Psychodynamisch Imaginative Traumatherapie (PITT; Reddemann 2011), das Eye Movement Desensitization and Reprocessing (EMDR; Shapiro 1999) sowie die Dialektisch-Behaviorale Therapie (DBT; Linehan 1993). Die Behandlung wird in drei Phasen gegliedert: Die zweiwöchige Phase 1 dient der Stabilisierung und Diagnostik. Die Phase 2 beinhaltet die Traumaexposition und findet in Form einer Intervallbehandlung statt, wobei ein Intervall mindestens sechs Wochen andauern sollte. Die dritte und letzte Phase umfasst die Themen Trauer und Neuorientierung. Entsprechend Panksepps Einteilung in ein Furcht- und ein Paniksystem wird auch auf die Stressregulation in der Psychotherapie der Station 9 ein Fokus gelegt. Das beschriebene traumazentrierte Therapiekonzept kann demnach in eine Anti-Furcht- (z. B. Traumasynthese mittels Expositionen aus PITT und EMDR) und eine Anti-Panik-Therapie (z. B. Imaginationen und „Innere-Kind-Arbeit“ aus PITT sowie Achtsamkeit aus DBT) unterteilt werden, denn beide Stresssysteme sind bei der komplexen PTBS beeinträchtigt (Müller und Sachsse 2009, Sachsse et al. 2002). Für die Einzelheiten des Therapiekonzepts der Station 9 sei auf Sachsses „Traumazentrierte Psychotherapie“ verwiesen (Sachsse 2004).

Zur Evaluation der Wirksamkeit dieses Therapieprogramms wurde der Therapieerfolg direkt sowie ein Jahr nach Therapieende anhand von Selbstbeurteilungsfragebögen und einem semistrukturierten Interview in einem naturalistischen Studiendesign bestimmt. Diese 1Jahres-Katamnese bescheinigt den Patientinnen der Station 9 im Vergleich zu einer mit herkömmlichen Therapieverfahren behandelten Kontrollgruppe signifikante und stabile Verbesserungen sowohl in traumaspezifischen Symptomen (z. B. Intrusionen, Dissoziationen, Vermeidung) als auch in allgemein psychischen Symptomen (z. B. allgemeine Stresssymptomatik, Häufigkeit von selbstverletzendem Verhalten, Anzahl an Hospitalisierungen). Besonders bemerkenswert ist, wie drastisch die Dauer von Krankenhausaufenthalten gegenüber Therapiebeginn sank: Zwei Patientinnen befanden sich zwar nach Entlassung immer noch 140 Tage jährlich in stationärer Therapie, die restlichen Patientinnen benötigten jedoch nur noch null bis vier Tage stationäre Behandlung im Jahr (Sachsse et al. 2006). Eine retrospektive Studie konnte die Ergebnisse der 1-Jahres-Katamnese auch im Langzeitverlauf bestätigen. Es zeigten sich Besserungen in verschiedenen Symptombereichen, bei der Stimmung und bzgl. der Hospitalisierungsrate, wenngleich „eine gewisse Vulnerabilität und Belastung“ bei den Patientinnen bestehen blieb (Müller und Sachsse 2010, S. 142). 


\subsubsection{Therapiewirkung auf die autonome Dysregulation}

Während die Therapiekonzepte für die PTBS hauptsächlich anhand von psychometrischen Scores, semistrukturierten Interviews und Indices zur Lebensqualität evaluiert werden, widmeten sich bislang nur wenige Studien der Frage, ob klinisch erfolgreiche Therapien auch eine Wirkung auf die autonome Dysregulation der PTBS zeigen. Die meisten der bisherigen Studien auf diesem Gebiet konzentrierten sich auf eine Betrachtung der Herzraten-Reaktivität bei traumaassoziierten Reizen vor und nach einer Therapie. Mehrmals wurde anhand von Verkehrsunfallopfern (mit PTBS) die kognitive Verhaltenstherapie (kVT) mit der supportiven Psychotherapie (sPT) und/oder mit einer Kontrollgruppe verglichen. Die Kontrollgruppe entsprach dabei Patienten auf einer Warteliste für eine Therapie. Dabei wies bei Blanchard et al. (2002) sowie bei Rabe et al. (2006) die Gruppe kVT eine signifikant größere Reduktion der HR-Reaktivität auf als die Gruppe sPT und die Kontrollgruppe. Karl et al. (2004) konnten dagegen anhand von EMG-Messungen bei allen therapierten Patienten (kVT und sPT) im Vergleich zur Kontrollgruppe eine signifikante Reduktion der Reaktivität auf traumaassoziierte Stimuli feststellen. Shalev et al. (1992) evaluierten anhand von Herzrate, EMG und Hautleitfähigkeit die systematische Desensibilisierung bei drei PTBS-Probanden. Auf therapierte traumatische Stimuli wurde nicht mehr mit einer verstärkten physiologischen Antwort reagiert, bei nicht therapierten Stimuli blieb die Reaktion weiterhin erhöht (Shalev et al. 1992). Nishith et al. (2003) untersuchten sechs Vergewaltigungsopfer mit PTBS, wobei fünf von einer kognitiven Verhaltenstherapie profitierten und eine die Therapie abbrach. Die fünf erfolgreich therapierten Patientinnen wiesen eine gegenüber vor der Therapie verringerte sympathische Dominanz im Sinne einer reduzierten LF/HF-Ratio während des REMSchlafes auf. Bei der Abbrecherin stieg dagegen der sympathische Einfluss an. Auch Griffin et al. (2012) wählten weibliche Opfer von sexueller und körperlicher Gewalt als Untersuchungsgruppe und konnten bei Therapierespondern die Verringerung einer Hyperreaktivität auf Schreckreize feststellen.

Diese beispielhaft beschriebenen Studien weisen somit allesamt daraufhin, dass eine Therapie einen Effekt auf die autonome Reaktivität bei der PTBS haben und die Herzrate während traumabezogener Stressreize diesen abbilden könnte. Die Forschung zu dieser Thematik steht jedoch noch in den Anfängen und weitere Studien sind für eine differenzierte Betrachtung einer möglichen Therapiewirkung notwendig. 


\section{Ziele und Hypothesen}

\subsection{Zielsetzung}

Die aktuelle Studienlage weist auf Veränderungen des autonomen und kardiovaskulären Systems im Zuge einer PTBS hin. Bisher stand die kardiovaskuläre Regulation von PTBSPatienten unter Ruhebedingungen und bei Exposition mit (traumaassoziierten) Stressoren im Fokus. Was den Langzeitverlauf anbelangt, betrachteten nur wenige Studien, inwiefern eine Therapie sich auch auf die autonome Regulation und Balance auswirkt. Die vorliegende Arbeit soll hier nun einen ersten Schritt darstellen, die psychische Symptomatik und kardiovaskuläre Regulation vor und drei Jahre nach einer PTBS-Therapie zu evaluieren.

Dazu umfasst die Studie zwei Messungen, eine Baseline-Messung 2009/2010 sowie eine Follow-up-Messung 2013. Zu beiden Messzeitpunkten wurde eine Stressfunktionsmessung durch den Task Force ${ }^{\circledR}$ Monitor bei PTBS-Patientinnen vorgenommen, zudem füllten diese psychometrische Fragebögen aus und gaben ihr subjektives Stressempfinden an. Zum Zeitpunkt Baseline wurden außerdem die gleichen Messdaten von einer Kontrollgruppe erhoben. Bei der Stressfunktionsmessung wurde die Regulation des autonomen Nervensystems in Ruhe sowie unter kognitiven und emotionalen Stress aufgezeichnet. Das Babyschreien als emotionaler Stressor wurde im ersten Teil der Studie (Baseline) erprobt. Die Reaktionen auf das Babyschreien sowie auf den kognitiven Stressor Rechnen sollen nun im Langzeitverlauf weiter evaluiert werden.

Die für die Auswertung der Stressfunktionsmessung ausgewählten kardiovaskulären Parameter haben sich alle in bisherigen Studien als gute und verlässliche Messgrößen für die autonome Dysregulation der PTBS erwiesen. Einzig die Herzrate wurde bereits zur Erfassung therapieassoziierter kardiovaskulärer Veränderungen eingesetzt; sie erschien hierfür als sensitiv. Alle anderen Parameter werden rein explorativ bezüglich ihrer Änderungssensitivität verwendet. Nur wenige Studien fokussierten sich bislang auf die Recovery einer Stressreaktion bei der PTBS, auch wenn es Hinweise gibt, dass eine pathologische Rückbildungsreaktion typisch für die PTBS und ein guter Marker für ein dysfunktionales ANS sein könnte. Bornschein hatte zudem eine verringerte Reagibilität als charakteristisch für die PTBS beschrieben (Bornschein 2014). Folglich soll daher die allgemeine Reagibilität unter Einbeziehung der Recovery als Verlaufsmarker für die Stressadaptation explorativ genutzt werden.

Bislang widmete sich keine Studie den kardiovaskulären Therapieeffekten speziell bei Patienten mit komplexen Traumafolgestörungen. Einzig Nishith et al. (2003) und Griffin et al. (2012) untersuchten bislang die psychophysiologische Therapiewirkung bei weiblichen Opfern sexueller und körperlicher Gewalt - inwieweit bei den Probandinnen eine kPTBSSymptomatik vorlag, geht jedoch nicht aus den Studien hervor. In der vorliegenden Arbeit kann dagegen dank umfassender klinischer Diagnostik zum Zeitpunkt Baseline eine sichere und differenzierte Einordnung der Probandinnen erfolgen. Somit wird erstmals gezielt eine 
Kohorte mit komplexen Traumafolgestörungen (bei überwiegend Typ-2-Traumatisierungen) für eine stressphysiologische Therapieevaluation herangezogen. So dient diese Studie auch einem besseren Verständnis für die psychophysiologischen Vorgänge bei den komplexen Traumafolgestörungen, die sich in vielerlei Hinsicht (u. a. auch in ihrem Therapieansprechen) von der einfachen PTBS unterscheiden.

\subsection{Hypothesen}

Folgende Hypothesen sollen mit dieser Arbeit untersucht werden:

1) Im Vergleich zur ersten Untersuchung (Baseline) geben die PTBS-Patientinnen während der zweiten Messung (Follow-up) ein niedrigeres subjektives Stressempfinden an.

2) Im Vergleich zur Baseline-Messung ist das allgemeine psychische Befinden der PTBSPatientinnen zum Zeitpunkt Follow-up besser.

3) Im Follow-up sind bei den PTBS-Patientinnen Unterschiede in der Aktivierung und Reagibilität des autonomen Nervensystems anhand von einigen ausgewählten kardiovaskulären Parametern gegenüber der Baseline-Untersuchung festzustellen.

a) Die PTBS-Patientinnen hatten während der Baseline-Untersuchung in Ruhebedingungen eine höhere kardiovaskuläre Aktivierung als die Kontrollgruppe gezeigt (Bornschein 2014). Im Follow-up nähern sich die Werte der PTBSPatientinnen den Werten der Kontrollgruppe an.

Dies heißt im Einzelnen:

- $\quad$ Die Herzrate (HR), der systolische Blutdruck (sBP) und der totale periphere Widerstandsindex (TPRI) sind bei Ruhebedingungen im Follow-up gegenüber der Baseline-Messung gesunken.

- Die Präejektionsperiode (PEP) und die Baroreflexsensitivität (BRS) sind bei Ruhebedingungen im Follow-up gegenüber der Baseline-Messung angestiegen.

b) Während der Baseline-Messung hatten die PTBS-Patientinnen im Vergleich zur Kontrollgruppe eine verringerte Reagibilität des autonomen Nervensystems während der verschiedenen Stressphasen gezeigt (Bornschein 2014). Diese Reagibilität ist bei dem Follow-up höher und damit näher an den Werten der Kontrollgruppe. Hierzu werden ebenfalls die Parameter HR, sBP, TPRI, PEP und BRS betrachtet. 
4) Die PTBS-Patientinnen, deren subjektives Stressempfinden sich im Vergleich zur Baseline-Messung besonders verringert hat, zeigen im Follow-up (gegenüber der Baseline-Messung) auch eine höhere Reagibilität der physiologischen Stressparameter (HR, sBP, TPRI, PEP und BRS).

5) Die PTBS-Patientinnen, deren psychisches Befinden sich im Vergleich zur BaselineUntersuchung besonders verbessert hat, verzeichnen im Follow-up (gegenüber der Baseline-Messung) auch eine höhere Reagibilität der physiologischen Stressparameter (HR, sBP, TPRI, PEP und BRS).

6) Die PTBS-Patientinnen, die sich länger in psychotherapeutischer stationärer Therapie befanden, sind durch besonders starke Verbesserungen in ihrem psychischen Befinden von Baseline zu Follow-up, durch eine besonders geringe subjektive Stresswahrnehmung im Follow-up im Vergleich zur Baseline-Messung und/oder durch eine besonders hohe Stressreagibilität der Parameter HR, sBP, TPRI, PEP und BRS im Follow-up im Vergleich zur Baseline-Messung gekennzeichnet. 


\section{Material und Methoden}

\subsection{Die Studie}

Die vorliegende Studie wurde in der Klinik für Psychosomatische Medizin und Psychotherapie der Universitätsmedizin Göttingen in den Jahren 2009 bis 2013 durchgeführt.

Mithilfe eines kardiovaskulären Funktionsmonitors, des Task Force ${ }^{\circledR}$ Monitors, wurde die Stressreagibilität des autonomen Nervensystems von PTBS-Patientinnen während eines mehrphasigen Stresstests zu zwei Zeitpunkten aufgezeichnet: zu Beginn der Therapie (Baseline-Untersuchung) und etwa drei Jahre später (Follow-up-Untersuchung). Gleichzeitig wurden das subjektive Stressempfinden während des Stresstests sowie aktuelle psychische Beschwerden anhand mehrerer psychometrischer Fragebögen erhoben.

Die Baseline-Untersuchung erfolgte in den Jahren 2009 und 2010. Hierbei wurde zugleich auch eine Kontrollgruppe untersucht. Das Untersuchungsteam bestand zu diesem Zeitpunkt aus den Projektleitern Prof. Dr. Ch. Herrmann-Lingen und Prof. Dr. U. Sachsse, zwei Doktorandinnen sowie dem medizinischen Team der Station 9 des Asklepios Fachklinikums Göttingen (Albrecht 2013). Die Follow-up-Untersuchung wurde im Laufe des Jahres 2013 durchgeführt. Hieran waren die Projektleiter Prof. Dr. Ch. Herrmann-Lingen und Prof. Dr. U. Sachsse sowie eine Doktorandin beteiligt.

Der Ethikantrag sowohl für die Baseline- als auch für die Follow-up-Untersuchung wurde 2009 unter dem Aktenzeichen 14/6/09 von der Ethikkommission der Medizinischen Fakultät der Universität Göttingen bewilligt.

Eine erste Auswertung der 2009 und 2010 erhobenen Daten fand bereits in zwei Dissertationen und einem Paper statt (Albrecht 2013, Bornschein 2014, Meyer et al. 2016). In der vorliegenden Dissertation soll nun der Schwerpunkt auf dem Langzeitverlauf der kardiovaskulären Stressparameter, auch in Bezug auf die psychometrischen Daten der PTBS-Patientinnen, liegen und ein Vergleich zwischen Baseline und Follow-up vorgenommen werden.

Die folgende Methodik hat ihren Schwerpunkt auf dem Follow-up, für eine umfassende Beschreibung der Methodik der Baseline-Untersuchung sei an dieser Stelle auf die Dissertationen von Albrecht (2013) und Bornschein (2014) verwiesen. Um eine Vergleichbarkeit der Daten zu gewährleisten, war der Untersuchungsablauf bei beiden Untersuchungszeitpunkten möglichst identisch. Überschneidungen in der Darstellung der Methodik der vorliegenden Dissertation und der Doktorarbeiten von Albrecht und Bornschein sind daher nicht zu vermeiden. 


\subsubsection{Die Stichprobe: Rekrutierung, Ein- und Ausschlusskriterien}

Bei der Rekrutierung 2009 befanden sich alle potentiellen Teilnehmerinnen der Studie für eine Diagnostikphase oder für das erste Therapieintervall auf der Station 9 des Asklepios Fachklinikums Göttingen. Einschlusskriterium war, dass eine Posttraumatische Belastungsstörung oder eine Borderline-Störung anhand von strukturierten Interviews und Fragebögen diagnostiziert worden sein musste und die Teilnehmerinnen zum Zeitpunkt der BaselineUntersuchung zwischen 18 und 60 Jahren alt waren. Die Eingrenzung des Altersbereichs diente der Kontrolle altersbedingter kardiovaskulärer Veränderungen. Ausschlusskriterien waren eine bekannte schwere Herzerkrankung, wie z. B. eine hochgradige Herzrhythmusstörung oder eine schwere Herzinsuffizienz, sowie eine diabetische Polyneuropathie.

Das rekrutierte Kollektiv umfasste 54 Patientinnen. Da von Anfang an ein Follow-up geplant war, wurde zu Beginn der Studie eine informierte Einverständniserklärung für die Studienteilnahme mit Baseline- und Follow-up-Untersuchung von den Teilnehmerinnen eingeholt. Dabei wurde vor allem in Hinblick auf die Follow-up-Messung darauf hingewiesen, dass ein Widerruf der Zustimmung jederzeit möglich ist. Hiervon machte jedoch keine Patientin Gebrauch. Vier der Patientinnen wurden in Absprache mit der Station 9 nicht für die Followup-Untersuchung kontaktiert, da bei diesen eine ablehnende Haltung gegenüber einer Kontaktaufnahme im Zuge der Studie erwartet wurde. Grundlage für diese Annahme war, dass mehreren Patientinnen aus unterschiedlichen Gründen keine Therapie bzw. keine Therapiefortsetzung auf der Station 9 angeboten worden war. Im Gegensatz zu den anderen Patientinnen hatten diese vier die Entscheidung jedoch nicht akzeptiert. Folglich wurden im Jahr 201350 Patientinnen angeschrieben und zum Follow-up nach Göttingen eingeladen.

Insgesamt wurden die Patientinnen maximal dreimal postalisch kontaktiert. Hierbei meldeten sich 26 Patientinnen zurück. Einige Patientinnen äußerten Bedenken, die Untersuchung könnte ihr psychisches Befinden verschlechtern, da sie im Gegensatz zur ersten Untersuchung nicht durch die stationäre Behandlung aufgefangen werden könnten. Daraufhin wurde in dem zweiten und dritten Anschreiben den Patientinnen ein Gesprächstermin bei Herrn Prof. Dr. Sachsse direkt im Anschluss an die Untersuchung angeboten. Zwei Patientinnen vereinbarten einen solchen Termin, beschlossen aber beide aufgrund ihres stabilen Befindens nach der Untersuchung, ihn nicht wahrzunehmen. Die restlichen 24 Patientinnen, die nicht am Follow-up teilnahmen, sagten entweder ab oder meldeten sich auch nach dem dritten Anschreiben nicht zurück.

Da ein Transport des Task Force ${ }^{\circledR}$ Monitors an die Wohnorte der Patientinnen nicht möglich war, mussten die Patientinnen für die Untersuchung nach Göttingen kommen. Dies bedeutete für manche Patientinnen einen langen Anreiseweg, sie wohnten zum Teil quer über Deutschland verstreut. 16 Patientinnen hatten eine Anreise über eine Stunde oder gaben an, dass sie sich durch die Anreise besonders gestresst gefühlt hätten. Die restlichen wohnten 
näher oder sogar in Göttingen. Fahrtkosten konnten erstattet werden, eine weitere Aufwandsentschädigung war jedoch nicht möglich. Die Patientinnen kamen je nach Wahl mit Auto, Zug, Mitfahrgelegenheit oder bei kurzen Entfernungen mit dem Taxi.

Zwei Patientinnen befanden sich gleichzeitig zur Follow-up-Teilnahme in einer stationären Therapie auf der Station 9 des Asklepios Fachklinikums Göttingen. Hier wurde darauf geachtet, dass die Untersuchung keine zeitliche Nähe zu einer Traumaexposition hatte. Eine weitere Patientin war zum Zeitpunkt der Messung zur Diagnostik auf einer neurologischen Station der Universitätsmedizin Göttingen aufgenommen.

\subsection{Material}

\subsubsection{Die Fragebögen}

Zur Erfassung der psychischen Beschwerden und Belastungen zum Zeitpunkt der Messung füllten die Patientinnen jeweils im Vorfeld der Untersuchungen mehrere Selbstbeurteilungsfragebögen aus. Während bei der Baseline-Untersuchung die psychometrischen Fragebögen den Patientinnen bei der Aufnahme auf die Station 9 ausgeteilt wurden, erhielten die Patientinnen sie bei der Follow-up-Untersuchung postalisch - mit der Bitte sie wenige Tage vor der Messung auszufüllen. Außerdem sollten einige allgemeine Daten erhoben werden, welches mit einem Fragebogen zu soziodemographischen Daten und Vorerkrankungen geschah. Dieser allgemeine Fragebogen wurde jeweils kurz vor der Messung von den Patientinnen beantwortet. Für das Follow-up wurde ein zusätzlicher Fragebogen konzipiert, in dem eine Auflistung der Therapien der letzten Jahre sowie der Medikation erfolgte. Der zusätzliche Fragebogen wurde den Patientinnen zusammen mit den psychometrischen Fragebögen im Vorfeld zugesandt. Im Folgenden werden die einzelnen Fragebögen vorgestellt.

\subsubsection{Symptom-Checkliste-90-R (SCL-90-R)}

Die Symptom-Checkliste-90-R (Derogatis et al. 1973) ist ein weltweit eingesetztes Selbstbeurteilungsinstrument, mit dem die subjektiv empfundene Beeinträchtigung durch körperliche und psychische Symptome bezogen auf die letzten sieben Tage erfragt wird (Franke 2014). Entwickelt wurde die SCL-90-R ursprünglich vor allem, um die psychischen Belastungen von Patienten, insbesondere von Psychotherapiepatienten, zu messen (Franke 2001). Inzwischen findet sie aber auch als Screeningverfahren für psychische Erkrankungen sowie als Outcome-Instrument in der klinischen Forschung Verwendung (Schmitz et al. 2000). In der Studie wurde die von Franke veröffentlichte deutsche Version verwendet (Franke 2000). In 90 Items wird das Ausmaß symptomatischer Belastungen jeweils mit einer fünfstufigen Likert-Skala (,überhaupt nicht“" bis „sehr stark“) beschrieben. Die Items werden zu den neun Skalen Aggressivität/Feindseligkeit, Ängstlichkeit, Depressivität, Paranoides Denken, Phobische Angst, Psychotizismus, Somatisierung, Unsicherheit im Sozialkontakt und Zwang- 
haftigkeit zusammengefasst. Drei globale Kennwerte spiegeln außerdem das Antwortverhalten über alle Items hinweg wider, indem sie über die grundsätzliche psychische Belastung (GSI), über die Intensität der positiven Antworten (PSDI) und über die Anzahl der belastenden Symptome (PST) Auskunft geben (Franke 2014). Die primären Skalen sowie die globalen Kennwerte werden mithilfe von alters- und geschlechtsabhängigen T-Normen ausgewertet und zur Beurteilung der Beeinträchtigungsschwere herangezogen. Die hierzu von Derogatis festgelegten Grenzwerte ermöglichen eine orientierende Aufteilung in „auffällig belastet“ und „nicht auffällig belastet“ (Franke 2014). Die Bearbeitungsdauer der Symptom-Checkliste-90-R beträgt etwa zehn bis fünfzehn Minuten.

Franke bescheinigt der SCL-90-R eine gute Reliabilität (Franke 2001), die Validität der einzelnen Skalen und die Eignung der SCL-90-R für das Screening auf bestimmte psychische Erkrankungen wird allerdings kontrovers diskutiert. Wird die SCL-90-R jedoch unidimensional zur Messung der allgemeinen symptomatischen Beeinträchtigung wie in dieser Studie eingesetzt, wird von einer guten Validität ausgegangen (Schmitz et al. 2000). Auch für Patienten mit Persönlichkeitsstörung wurde der Symptom-Checkliste-90-R die Aussagekraft bescheinigt, welches aufgrund der komorbiden Borderline-Störung mancher PTBS-Patientinnen in der Studie entscheidend ist (Tritt et al. 2006).

\subsubsection{Beck-Depressions-Inventar (BDI)}

Das Beck-Depressions-Inventar (BDI) wurde von Beck et al. (1961) entwickelt und misst die Schwere einer aktuellen depressiven Symptomatik. In dieser Studie wird die deutsche Übersetzung von Hautzinger eingesetzt, die auf einer 1978 modifizierten Version des BDI beruht (Hautzinger et al. 1994). Der Fragebogen ist weltweit etabliert und wird als Selbstbeurteilungsinstrument besonders für die Überprüfung von Therapieverläufen bei leicht bis mittelschwer depressiven Patienten eingesetzt (Richter 1991). Das BDI umfasst 21 Items, die Beck aufgrund von klinischen Beobachtungen formulierte (Steer et al. 1986). Jedes Item erfragt ein typisches Symptom einer Depression: (A) traurige Stimmung, (B) Pessimismus, (C) Versagen, (D) Unzufriedenheit, (E) Schuldgefühle, (F) Strafbedürfnis, (G) Selbsthass, (H) Selbstanklagen, (I) Selbstmordimpulse, (J) Weinen, (K) Reizbarkeit, (L) sozialer Rückzug und Isolierung, (M) Entschlussunfähigkeit, (N) Negatives Körperbild, (O) Arbeitsunfähigkeit, (P) Schlafstörungen, (Q) Ermüdbarkeit, (R) Appetitverlust, (S) Gewichtsverlust, (T) Hypochondrie, (U) Libidoverlust. Anhand der Intensität und Häufigkeit des Auftretens in der letzten Woche werden die Items jeweils in eine vierstufige Skala (0 bis 3) eingeordnet. Die Bearbeitungszeit beträgt etwa zehn Minuten. Die Werte aller Items werden zu einem globalen Summenwert aufsummiert, mit welchem nun eine Auswertung anhand von Cut-off-Werten vorgenommen werden kann.

Die Testgütekriterien des BDI wurden durch zahlreiche Studien überprüft (Kühner et al. 2007). Richter et al. (1998) analysierten die vorangegangen Validitätsstudien des BDI und nennen die hohe innere Konsistenz, die hohe Inhaltsvalidität, die hohe differentielle Validität bei der Unterscheidung von depressiven und nicht depressiven Probanden sowie die gute 
Änderungssensitivität als Vorteile. Aber auch einige Probleme, u. a. das Fehlen repräsentativer Normen, eine umstrittene Faktorvalidität und eine geringe Diskriminanzvalidität gegenüber Angst, werden beschrieben.

\subsubsection{Impact of Event Scale (IES-R)}

Die Impact of Event Scale (IES-R) misst den subjektiven Stress von Personen, die ein schwerwiegendes Lebensereignis in ihrer Vergangenheit erlebt haben, und ist somit insbesondere für Patienten mit Posttraumatischer Belastungsstörung konzipiert. Die erste Version der Impact of Event Scale wurde von Horowitz et al. (1979) publiziert, während die revidierte Form (IES-R) 1996 folgte (Weiss und Marmar 1997). Die hier verwendete deutsche Version der revidierten Form erschien nur zwei Jahre später (Maercker und Schützwohl 1998). Inzwischen erfreut sich die IES-R weltweit großer Beliebtheit. Die 22 Items werden drei Subskalen zugeordnet: Intrusion, Vermeidung und Übererregung. Hier spiegelt sich das in Kapitel 1.1.1 beschriebene Konzept wider, nach dem sich Stress nach einem traumatischen Erlebnis häufig in diesen drei Reaktionsmustern äußert, wobei die individuelle Ausprägung sehr unterschiedlich sein kann (Maercker 2003a). Die Antwortskalierung ist vierstufig und es wird angegeben, wie häufig in den letzten sieben Tagen ein in dem Item beschriebenes Ereignis aufgetreten ist. Die Bearbeitungszeit liegt zwischen sieben und fünfzehn Minuten. Zur Auswertung werden die drei Subskalen angegeben, es wird kein Gesamtskalenwert errechnet. Folgende Formel kann jedoch herangezogen werden, die alle drei Skalenwerte miteinbezieht (Maercker und Schützwohl 1998, S. 138):

$\mathrm{X}=(-0,02 \mathrm{x}$ Intrusion $)+(0,07 \times$ Vermeidung $)+(0,15 \times$ Übererregung $)-4,36$

Ergibt sich für X eine positive Zahl, besteht die Verdachtsdiagnose einer Posttraumatischen Belastungsstörung.

Der IES-R wurde sowohl in der englischen als auch in der deutschen Version eine zufriedenstellende Reliabilität, Validität und diagnostische Sensitivität bescheinigt, auch wenn die faktorielle Validität umstritten bleibt (Maercker und Schützwohl 1998, Creamer et al. 2003).

\subsubsection{Fragebogen zu dissoziativen Symptomen (FDS)}

Der Fragebogen zu dissoziativen Symptomen (Freyberger et al. 1998) ist die deutsche Adaptation der DES, der Dissociative Experience Scale (Bernstein und Putnam 1986), und dient der quantitativen Erfassung dissoziativer Phänomene. Während die DES dissoziative Symptome in den Bereichen Gedächtnis, Kognition, Bewusstsein und Identität abbildet und damit bezüglich der Kriterien einer dissoziativen Störung dem DSM-III-R und DSM-IV (American Psychiatric Association 1987, 1994) entspricht, wurde der FDS zusätzlich an die ICD-10-Klassifizierung angepasst. In der ICD-10 wird eine dissoziative Störung nicht nur über die Manifestation auf psychisch-kognitiver Ebene definiert, sondern es werden auch pseudoneurologische Körpersymptome bei der Diagnose miteinbezogen. Aufgrund dieser Erweiterung um die Symptome in den Bereichen Sensorik, Sensibilität und Motorik umfasst 
der FDS 44 Items - 16 Items mehr als die DES. Wie sehr ein Item zutrifft wird von den Patientinnen anhand einer Skala von 0 bis $100 \%$ angegeben, wobei eine Abstufung in Zehnerschritten möglich ist. Ein spezieller Zeitraum bezüglich des Auftretens der Symptome wird nicht vorgegeben, es wird nach dem allgemeinen Vorkommen gefragt. Zur Auswertung werden ein Gesamtskalenwert des FDS sowie die drei Subskalen dissoziative Amnesie, Tendenz zu imaginativen Erlebnisweisen und Depersonalisation/Derealisation berechnet. Zudem kann aus dem FDS auch eine Subskala für pseudoneurologische Konversionssymptome sowie eine allgemeine Subskala für die ICD-10-spezifischen dissoziativen Symptome bestimmt werden. Beschränkt man sich auf die Addition der 28 DES-Items, kann auch der Gesamtskalenwert für die DES angegeben werden.

Eine gute Reliabilität wie auch eine gute Validität wurden sowohl der DES als auch dem FDS mehrfach nachgewiesen (Freyberger et al. 1998, Rodewald et al. 2006, Van Ijzendoorn und Schuengel 1996).

\subsubsection{Borderline-Persönlichkeits-Inventar (BPI)}

Das Borderline-Persönlichkeits-Inventar (BPI) ist ein Selbstbeurteilungsinstrument, mit dem die Diagnose einer Borderline-Persönlichkeitsstörung nahegelegt werden kann (Leichsenring 1997). Der vor allem im Screening genutzte Fragebogen umfasst 53 mit Ja oder Nein zu beantwortende Items und basiert auf Kernbergs Konzept der Borderline-Persönlichkeitsorganisation (Kernberg 1967, Kernberg 1988). Daher beinhaltet er Skalen zur Identitätsdiffusion, zu primitiven Abwehrmechanismen/Objektbeziehungen, zur mangelhaften Realitätsprüfung sowie zur Angst vor Nähe bzw. Verschmelzung. Er ist damit auch vereinbar mit den Kriterien des Diagnostic Interview for Borderlines (Gunderson et al. 1981), den DSM-IVKriterien (American Psychiatric Association 1994) sowie der ICD-10-Definition für eine Borderline-Persönlichkeitsstörung (Leichsenring und Chabrol 2006). Die Skalen wurden faktorenanalytisch konstruiert, ein aus den 20 trennschärfsten Items ermittelter Wert (Cut20-Wert) weist bei Überschreiten eines definierten Cut-offs auf die Diagnose der Störung hin. Zudem können die Skalenwerte sowie der Cut-20-Wert in T-Normen und Prozentränge transformiert werden, Vergleichsstichproben sind hierfür beispielsweise Nicht-Patientinnen und Borderline-Patienten (Leichsenring 1997). Die Bearbeitungszeit beträgt etwa 20 Minuten. Die Reliabilität des BPI ist zufriedenstellend und bisher erhobene Daten sprechen für eine ausreichende bis gute Validität (Leichsenring und Chabrol 2006, Lindner 2001).

\subsubsection{Borderline-Symptom-Liste (BSL)}

Die Borderline-Symptom-Liste (BSL) ist eine Selbstbeurteilungsskala zur Quantifizierung der intrapsychischen Belastungen von Borderline-Patienten (Bohus et al. 2001). Dabei basiert sie auf dem Kriterienkatalog des DSM-IV (American Psychiatric Association 1994) sowie auf der revidierten Version des Diagnostic Interview for Borderlines (Gunderson et al. 1981), auch die Berichte einiger Experten und zahlreiche Patientenaussagen wurden berücksichtigt. Während für die Diagnostik einer Borderline-Störung bereits einige gut etablierte Instrumente 
existieren, soll die BSL eine Schweregraderfassung von störungsspezifischen Symptomen ermöglichen und kann damit auch zu Verlaufskontrollen in Psychotherapie und Forschung herangezogen werden (Bohus et al. 2001). Die insgesamt 95 Items umfassen die Skalen Selbstwahrnehmung, Affektregulation, Autoaggression, Dysthymie, soziale Isolation, Intrusionen und Feindseligkeit und werden bezogen auf die letzte Woche anhand einer 5-stufigen Likert-Skala bewertet. Außerdem wird mittels einer visuellen Analogskala (von 0 bis $100 \%$ ) die aktuelle psychische Befindlichkeit erfasst. Eine BSL-Ergänzungsskala mit elf Items fragt nach aktuellen dysfunktionalen Verhaltensweisen. Die Auswertung erfolgt anhand der Skalenmittelwerte sowie eines Gesamtscores, die Prozenträngen zugeordnet werden und somit eine Einordnung der Symptomlast in Bezug auf eine Vergleichsstichprobe ermöglichen. Für die BSL besteht die Vergleichsstichprobe aus 308 Borderline-Patientinnen. Die Validität und Reliabilität des Fragebogen wurden nachgewiesen und als gut bis sehr gut eingeschätzt (Bohus et al. 2007).

\subsubsection{Allgemeine Fragebögen und Erhebung klinischer Daten}

Ziel des allgemeinen Fragebogens (s. Anhang, Kap. 8.7) war es, soziodemographische Daten zu den Patientinnen zu erheben. Dabei wurde bei der Baseline-Untersuchung und bei dem Follow-up der gleiche Fragebogen verwendet und direkt vor der Messung ausgefüllt. Gefragt wurden nach Alter, Größe, Gewicht, Beziehungsstatus, Wohnsituation, Kinder, körperliche Betätigung, Alkoholkonsum, Tabakabusus, Schul- und Ausbildungsabschluss, Erwerbstätigkeit, Medikamenteneinnahme sowie Vorerkrankungen. Außerdem gaben die Patientinnen an, ob sie sich in der letzten Zeit besonders gestresst gefühlt hatten.

Im Gegensatz zu der Baseline-Untersuchung wurde bei dem Follow-up ein weiterer Fragebogen zu Therapien und Medikation ausgegeben (s. Anhang, Kap. 8.8). Dieser Fragebogen wurde den Patientinnen zusammen mit den psychometrischen Fragebögen bereits im Vorfeld zugeschickt und somit zu Hause ausgefüllt. Es galt die stationär psychotherapeutischen bzw. psychiatrischen, die ambulant psychotherapeutischen oder psychiatrischen sowie die stationär somatischen Behandlungen seit der Baseline-Untersuchung, also in den Jahren 2009/2010 bis 2013, in eine Tabelle einzutragen. In einer weiteren Tabelle wurde die aktuelle Medikation noch einmal genauer erfragt - hier sollten die Patientinnen auch Dosierungen und das Einnahmeschema angeben. Für die im Vorfeld zu Hause auszufüllenden Fragebögen (inklusive der psychometrischen Fragebögen) ist von einer Gesamtbearbeitungszeit von ungefähr 1 Stunde und 45 Minuten auszugehen.

Weitere klinische Daten wie psychiatrische Diagnosen, somatische Erkrankungen, Suchtverhalten, Art der Traumatisierung, selbstverletzendes Verhalten sowie die damalige Medikamenteneinnahme wurden zum Zeitpunkt der Baseline-Untersuchung mithilfe der Krankenakten des Asklepios Fachklinikums Göttingen gewonnen (Albrecht 2013). 


\subsubsection{Der Task Force ${ }^{\circledR}$ Monitor}

Die folgenden Ausführungen über den Task Force ${ }^{\circledR}$ Monitor beziehen sich - wenn nicht anders angegeben - auf die Veröffentlichungen der Arbeitsgruppe Jürgen Fortin in den Jahren 1998 bis 2013 (Fortin et al. 1998, 2001, 2006a, 2006b, 2013; Gratze et al. 1998), auf das Manual des Task Force ${ }^{\circledR}$ Monitors (CNSystems Medizintechnik AG 2010) sowie auf die Dissertation von Jürgen Fortin (Fortin 2007).

Der Task Force ${ }^{\circledR}$ Monitor (TFM) der Firma CNSystems Medizintechnik AG dient der nichtinvasiven Messung autonom und hämodynamisch kontrollierter Parameter. Dabei zeichnet sich der TFM auch durch seine kontinuierliche beat-to-beat Aufzeichnung in Echtzeit aus, was ihn für das Erkennen von schnellen und kurzzeitigen Veränderungen im kardiovaskulären System besonders nützlich macht.

Der Task Force ${ }^{\circledR}$ Monitor kombiniert gleich drei Technologien der Firma CNSystems:

- CNAP (continuous non-invasive arterial pressure) beschreibt die beat-to-beat-Messung des Blutdrucks mittels der nicht-invasiven vascular unloading-Technik.

- CNCO (continuous non-invasive cardiac output and stroke volume) wird das System genannt, das mittels Impedanzkardiographie (IKG) ebenfalls beat-to-beat Schlagvolumen und Herzzeitvolumen bestimmt.

- CNAA (continuous non-invasive assessment of autonomic function) ist ein Softwarepaket, das in Echtzeit weitere autonome Parameter wie die Herzraten- und Blutdruckvariabilität und die Baroreflexsensitivität aus den gemessenen Daten errechnet.

Der Task Force ${ }^{\circledR}$ Monitor zeichnet zudem ein bipolares 2-Kanal-EKG (Einthoven I und II) über vier am Rumpf angebrachte Elektroden auf. Durch eine Abtastfrequenz von $1000 \mathrm{~Hz}$ sowie spezielle Algorithmen wird eine QRS-Detektion ermöglicht, die eine Fehlerrate von unter zwei Prozent aufweist. Eine oszillometrische Blutdruckmessung eicht die mittels CNAP-gemessenen Werte und steuert so absolute Zahlenwerte bei. Dabei wird eine Messgenauigkeit von $+/$ - $5 \mathrm{mmHg}$ erreicht. Alle Messdaten werden von dem TFM auf einen angeschlossenen Laptop übertragen, wo sie tabellarisch und graphisch dargestellt werden.

Insgesamt werden folgende Parameter direkt von dem TFM angezeigt und gespeichert:

- RR-Intervall (RRI) in ms und Herzrate (HR) in bpm

- Systolischer, diastolischer und mittlerer arterieller Blutdruck (sBP, dBP und mBP) in $\mathrm{mmHg}$ mittels CNAP und der oszillometrischen Blutdruckmessung

- Mittlere Basisimpedanz des Thorax Zo in Ohm, abgeleitet aus dem IKG

- Schlagvolumen (SV) in $\mathrm{ml}$ und Schlagindex (SI) in $\mathrm{ml} / \mathrm{m}^{2}$ : Der SI ist das SV normiert auf die Körperoberfläche (body surface area = BSA).

- Herzzeitvolumen (HZV) in $1 /$ min und Herzindex $(\mathrm{HI})$ in $1 /\left(\min ^{*} \mathrm{~m}^{2}\right)$ : Der HI ist das HZV normiert auf die BSA. 
- Totaler peripherer Widerstand (TPR) in dyn*s $/ \mathrm{cm}^{5}$ und totaler peripherer Widerstandsindex (TPRI) in dyn* ${ }^{*} \mathrm{~m}^{2} / \mathrm{cm}^{5}$ : Der TPRI ist der TPR normiert auf die BSA.

- Thorakaler Flüssigkeitsinhalt (TFC, thoracic fluid content) in $1 / \mathrm{kOhm}$, abgeleitet aus dem IKG

- Acceleration Index (ACI) in 100/ $\mathrm{s}^{2}$ : ist die maximale Beschleunigung des ImpedanzSignals (zweite Ableitung) zwischen dem Öffnen der Aortenklappe und DZ/ $\mathrm{dt}_{\max }$. $\mathrm{DZ} / \mathrm{dt}_{\max }$ ist dabei ein vom IKG abgeleiteter Wert, der den maximalen systolischen Fluss anzeigt.

- Kontraktilitätsindex (IC, index of contractility) in 1000/s: DZ/ $\mathrm{dt}_{\max }$ normiert, repräsentiert den maximalen Blutfluss während des Auswurfs des linken Ventrikels

- Präejektionsperiode (PEP) in ms: Zeit zwischen der R-Zacke (aus dem EKG) und dem Öffnen der Aortenklappe (aus dem IKG)

- Linksventrikuläre Ejektionszeit (LVET) in ms: Zeit zwischen Öffnen und Schließen der Aortenklappe

- Linksventrikulärer Arbeitsindex (LVWI, left ventricular work index) in $\mathrm{kg} * \mathrm{~m} / \mathrm{m}^{2}$ : repräsentiert die Arbeit, die der linke Ventrikel mit jedem Herzschlag leistet, bezogen auf die BSA

- Spektralanalyse der Herzraten- und Blutdruckvariabilität (HRV und BPV): VLF (very low frequencies), LF (low frequencies) und HF (high frequencies) werden unterschieden und in absoluten $\left[\mathrm{ms}^{2}, \mathrm{mmHg}^{2}\right]$ oder relativen [\%] Einheiten angegeben.

- Baroreflexsensitivität (BRS) in ms/mmHg: wird mithilfe der Sequenz-Methode (Di Rienzo et al. 2001) bestimmt. Eine sog. „up-Sequenz“ (bzw. „down-Sequenz“) ergibt sich, wenn gleichzeitig zu einem Anstieg (Abfall) des systolischen Blutdrucks um mind. $1 \mathrm{mmHg}$ bei drei oder mehr aufeinander folgenden Herzschlägen das RRIntervall um mindestens $4 \mathrm{~ms}$ größer (kleiner) wird. Aus allen detektierten Barorezeptorsequenzen, also sowohl aus den $u p$-, als auch aus den down-Sequenzen, wird die mittlere Steigung dieser Sequenzen errechnet (total slope mean). Dieser Parameter wird im Folgenden als Maß für die Baroreflexsensitivität verwendet.

$\mathrm{Zu}$ allen Messsystemen des Task Force ${ }^{\circledR}$ Monitors liegen gute und umfangreiche Evaluationen vor, die dem TFM auch im Vergleich zu älteren, bereits etablierten Technologien gute Ergebnisse bilanzieren. Eine Zertifizierung durch den TÜV Österreich (CE 0408) erfolgte im Jahre 2000 und seit 2002 liegt auch eine Zulassung durch die amerikanische FDA vor.

\subsubsection{Prinzipien der CNAP-Messung}

CNAP (Fortin et al. 2013, Fortin 2007) beschreibt ein Messsystem, mit dem kontinuierlich und in Echtzeit der Blutdruck über eine Fingermanschette aufgezeichnet wird. Dabei basiert das Messprinzip auf der vascular unloading-Technik (Prinzip der entspannten Gefäßwand). Hierbei wird das Blutvolumen der Fingerarterien plethysmographisch mit einer Infrarotleuchte bestimmt und durch einen variablen Außendruck über die Fingermanschette konstant gehalten. Blutdruckschwankungen in den Fingerarterien führen zu einer Anpassung des 
Außendrucks. Das Lichtplethysmogramm zeigt nun keine Pulsationen mehr an, das Blutvolumen ist konstant. Somit stimmt der Außendruck sehr genau mit dem intraarteriellen Blutdruck überein.

Während bei den vascular unloading-Systemen gemäß dem Peñáz-Verfahren (Peñáz et al. 1976) in der Regel nur ein einzelner Regelkreis zum Einsatz kommt, wurden bei der CNAPTechnik mehrere ineinandergreifende Regelkreise eingeführt. Dadurch sollte das PeñázVerfahren verbessert und eine ununterbrochene Langzeitmessung möglich werden. Eine weitere Besonderheit der CNAP-Technik ist die Eichung mittels einer oszillometrischen Blutdruckmessung am kontralateralen Arm, die in wählbaren Intervallen, etwa alle fünf Minuten, absolute Werte zur Verfügung stellt.

Da eine Fingervenenkompression bei dieser Art der Blutdruckmessung nicht zu vermeiden ist, wurde außerdem eine Flying-V-Fingermanschette entwickelt. Hiermit kann manuell ein Fingerwechsel während der Messung vorgenommen werden.

\subsubsection{Prinzipien der CNCO-Messung}

Die CNCO-Technik beruht auf der Impedanzkardiographie (IKG). Bei der Impedanzkardiographie wird der Thorax als gleichmäßiger elektrischer Leiter, gefüllt mit Blut, definiert. In diesem Modell werden die mit der Herzaktion synchronen Impedanzänderungen des Thorax allein durch die Volumenänderungen des kardiovaskulären Systems verursacht (Raaijmakers et al. 1999). So können Schlagvolumen und damit auch das HZV aus den Impedanzänderungen errechnet werden. Zusammen mit der Aufzeichnung der Herzaktion aus dem EKG sowie dem Blutdruck ist das IKG die Grundlage für die Bestimmung weiterer Parameter wie der Präejektionsperiode und des totalen peripheren Widerstands. Dank einer speziellen Software des TFM werden diese Parameter in Echtzeit angezeigt.

Die CNCO wurde mit dem Goldstandardverfahren Thermodilution verglichen und erwies sich bei der Bestimmung des Herzzeitvolumens als genauso verlässlich wie die Thermodilution (Fortin 2007).

\subsubsection{Auswahl der zu analysierenden Parameter}

Der Task Force ${ }^{\circledR}$ Monitor zeichnet eine Vielzahl an Parametern auf, daher müssen zur Wahrung der Übersichtlichkeit die Analysen auf einige wenige Parameter beschränkt werden. Anhand der Erfahrungen aus den vorausgegangenen Dissertationen zu der Baseline-Untersuchung (Albrecht 2013, Bornschein 2014) wurden fünf Parameter ausgewählt:

- $\quad$ Herzrate (HR)

- Systolischer Blutdruck (sBP)

- Totaler peripherer Widerstandsindex (TPRI)

- Präejektionsperiode (PEP)

- Baroreflexsensitivität (BRS) 
Während sBP und TPRI sowohl die $\alpha$-adrenergen als auch die $\beta$-adrenergen Komponenten der autonomen Stressreaktion abbilden, sind HR und PEP v. a. dem $\beta$-adrenergen System zuzuordnen. Die HR steht zudem gleichzeitig auch unter vagaler Steuerung. Die BRS gibt Auskunft über den Einfluss des Parasympathikus und wird daher als vagales Maß herangezogen. Somit sind alle wichtigen kardiovaskulären Stressregulationssysteme berücksichtigt.

\subsection{Versuchsplan}

Bei der vorliegenden Studie handelt es sich um ein quasiexperimentelles Prä-Post-Design mit einer Messwiederholung auf drei Faktoren: Untersuchungszeitpunkt (zweistufig; zu Beginn vs. drei Jahre nach der stationären PTBS-Behandlung) x Phase (zweistufig, Ruhe vs. Stress) x Stressor (zweistufig, Rechnen vs. Babyschreien). Eine Permutation der experimentellen Stresstests zur Kontrolle von Reihenfolgeeffekten wurde nicht vorgenommen. Zusätzlich können Vergleiche mit einer alters- und geschlechtsangepassten, gesunden Kontrollgruppe gezogen werden.

Der Stresstest umfasste eine Phase Rechnen als kognitiven Stressor mit Herausforderungscharakter und eine Phase Babyschreien als emotionalen Stressor. Für den Stressor Rechnen wurde der mental-arithmetic-stress-Test durchgeführt, bei dem die Patientinnen fünf Minuten lang immer wieder die Zahl 13 subtrahieren mussten; die Startzahl war 3505 (Lache 2006). Es wurde hierbei, je nach Leistungsfähigkeit der Patientin, Zeitdruck erzeugt; außerdem fand eine Korrektur von falschen Ergebnissen statt. So wurde auch bei Patientinnen mit guter Rechenleistung die Wirkung dieses Stressors sichergestellt. Der kognitive Stressor Rechnen wurde bereits in vielen Studien eingesetzt, sodass hier ein hoher Erfahrungswert vorherrscht (Kop et al. 2004, Ironson et al. 1992). Der emotionale Stressor Babyschreien wurde dagegen mit der Baseline-Untersuchung dieser Studie als vergleichsweise unerforschter Stressor „erprobt". Das Babyschreien ist ein Bindungsstressor und wurde insbesondere aufgrund des klinischen Eindrucks ausgewählt, wonach für PTBS-Patientinnen ein besonders starkes Ansprechen auf diesen Stressor zu erwarten war. Die Ergebnisse der Baseline-Untersuchung zeigten sich hierbei als vielversprechend (Albrecht 2013, Bornschein 2014). Die Patientinnen bekamen in dieser Stressphase fünf Minuten lang über Kopfhörer eine einheitliche Tonaufnahme mit Babyschreien vorgespielt.

\subsection{Versuchsablauf}

Die Untersuchung bestand aus einer etwa halbstündigen Messung am Task Force ${ }^{\circledR}$ Monitor. Um eine Vergleichbarkeit der Daten sicherzustellen, wurde das Follow-up bezüglich des Messablaufs und der Messbedingungen wie die Baseline-Untersuchung gestaltet.

Das Follow-up fand in der Universitätsmedizin Göttingen in einem Raum der Klinik für Psychosomatische Medizin und Psychotherapie statt. Der Untersuchungsraum war ein anderer als noch bei der Baseline-Untersuchung, es wurde jedoch versucht, auch hier einen kom- 
fortablen Sitzplatz und eine ähnlich angenehme Atmosphäre zu ermöglichen. Die Patientinnen wurden in der Regel vom Eingang der Universitätsmedizin Göttingen abgeholt und zu dem Untersuchungsraum begleitet. Nachdem sich die Patientinnen in dem Untersuchungsraum gesetzt hatten, wurden noch einige Minuten bis zur Messung abgewartet, um zu vermeiden, dass Stress durch die Anreise einen Einfluss auf die Untersuchung nehmen konnte. Die Patientinnen füllten in dieser Zeit den allgemeinen Fragebogen zu den soziodemographischen Daten aus (s. Kap. 3.2.1.7).

In Kapitel 3.2.2 wurde der Task Force ${ }^{\circledR}$ Monitor eingehend beschrieben, daher wird an dieser Stelle nur auf die praktische Durchführung der Messung eingegangen. Für die Untersuchung saßen die Patientinnen, lange Oberbekleidung musste zur Anbringung der Elektroden abgelegt werden. Für die IKG-Messung wurde eine Elektrode am Nacken, zwei bilateral auf Höhe des Xiphoids sowie eine Neutralelektrode am rechten Unterschenkel der Patientin befestigt. Zudem wurden vier EKG-Elektroden am Rumpf der Patientin angebracht. An der rechten Hand wurde eine Flying-V-Fingermanschette für die beat-to-beat-Blutdruckmessung und am linken Oberarm eine oszillometrische Blutdruckmanschette angelegt. Nachdem alle Elektroden sowie die Finger- und Oberarmmanschette mit dem Task Force ${ }^{\circledR}$ Monitor verbunden waren, erfolgte die Kalibrierung der Messkanäle. Nach einigen Minuten Kalibrierung konnte schließlich die Messung gestartet werden.

Abbildung 1 gibt einen Überblick über den Messablauf. Die Messung kann in sechs Phasen unterteilt werden, wobei sich „Ruhephasen“ und „Stressphasen“ abwechseln. Nach einer ersten Ruhephase (Ruhe 1) wurde mit der Phase Rechnen der kognitive Stressor gesetzt. Eine weitere Ruhephase (Ruhe 2) folgte, bevor der emotionale Stressor in Form von Babyschreien zum Einsatz kam. Nach einer erneuten Ruhephase (Ruhe 3) fand der letzte Messabschnitt statt - die Entspannungsmusik/Musik. Die Patientinnen hörten hierzu die Mondscheinsonate (Klaviersonate Nr.14) von Ludwig van Beethoven. Mit dieser Phase endete die Task Force ${ }^{\circledR}$ Monitor-Messung. Alle Messphasen waren fünf Minuten lang, mit Ausnahme der Phase Ruhe 2, die sechs Minuten andauerte. Zweimal (in den Phasen Ruhe 2 und Ruhe 3) wurde bei der plethysmographischen Blutdruckmessung der Finger gewechselt, da es sonst zu Durchblutungsstörungen am gemessenen Finger kommen kann. Hierbei setzt die kontinuierliche Blutdruckmessung kurz aus. In der Phase Ruhe 1 und jeweils kurz nach den Phasen Rechnen, Babyschreien und Musik wurde das aktuelle subjektive Stressempfinden erfragt. Die Patientinnen gaben dabei ihr Stresslevel auf einer Analogskala an (0 = gar kein Stress, 10 = größter vorstellbarer Stress). Zudem wurden vegetative Reaktionen wie Weinen oder dissoziative Symptome (starrer Blick, körperfremdes Gefühl) mit dem jeweiligen Zeitpunkt notiert. 


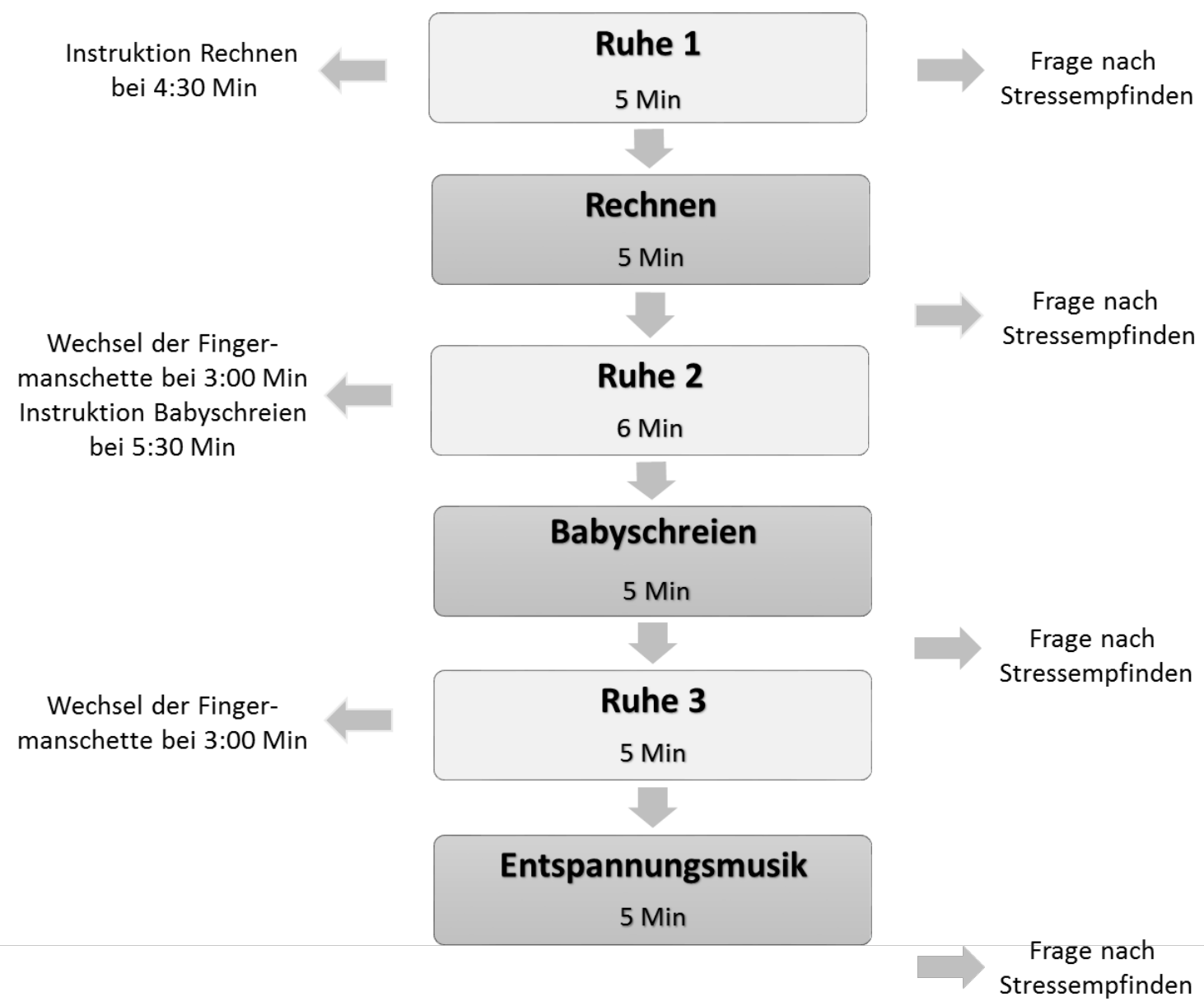

Abbildung 1: Messphasen während der Task Force Monitor®-Messung

\subsection{Statistik}

Die gesamte statistische Auswertung sowie die Erstellung der Graphen erfolgten mit den IBM SPSS Statistics Versionen 22 bis 24. Auf eine graphische Überprüfung auf Plausibilität, mit der z. B. Ausreißerwerte detektiert werden sollten, folgte die Prüfung auf Normalverteilung. Hierzu wurde die Differenz aus den Variablen der beiden Messzeitpunkte gebildet (Baseline - Follow-up) und diese auf Normalverteilung getestet. Als Kriterium wurde aufgrund der kleinen Stichprobengröße der Shapiro-Wilk-Test gewählt. Zusätzlich wurden Histogramme und QQ-Plots betrachtet. Um systematische Unterschiede aufzudecken, wurde eine Gegenüberstellung des Follow-up-Kollektivs mit den restlichen Patientinnen der Baseline-Untersuchung, die nicht an dem Follow-up teilgenommen hatten, vorgenommen. Dabei kam ein unabhängiger t-Test bzw. bei kategorialen Daten ein Chi-Quadrat-Test zum Einsatz.

Für die Bildung der $\Delta$ R-Scores als Maß für die Änderung der Reagibilität (s. Kap. 3.5.1) wurde die Bezugsgröße „Ruhebedingungen“ im Sinne einer Phase mit möglichst geringer 
kardiovaskulärer Aktivierung benötigt. Nur so konnte zusammen mit den beiden Stressphasen die maximale Stressreagibilität berechnet werden. Hierfür wurden die Phasen Ruhe 1 und Musik mithilfe einer 2 × 2 ANOVA für Messwiederholungen bezüglich der in Kapitel 3.2.2.3 festgelegten Parameter verglichen. Nachdem die $\Delta$ R-Scores bestimmt wurden, erfolgte auch hier eine graphische Betrachtung mittels Boxplots zur Detektion von Ausreißer- und Extremwerten.

Die deskriptive Betrachtung des Kollektivs geschah unter Angabe von prozentualen und absoluten Häufigkeiten sowie von Mittelwerten und Standardabweichungen bei metrischen Daten. Die Auswertung des subjektiven Stressempfindens über die Zeit wurde anhand von abhängigen t-Tests sowie - zur Einbeziehung des Faktors Phase - anhand einer 2 x 4 ANOVA für Messwiederholungen durchgeführt. Für die psychometrischen Fragebögen wurden die Gesamtwerte sowie die Subskalen mittels abhängigen t-Tests bzw. mittels Wilcoxon-Vorzeichen-Rang-Tests bei fehlender Normalverteilung zu den beiden Messzeitpunkten verglichen. Als ein erster Schritt zur Analyse der TFM-Daten wurde eine deskriptive Betrachtung der Parameter Herzrate, systolischer Blutdruck, TPRI, PEP und BRS im Verlauf der Stressmessung vorgenommen. Dazu wurden die Phasen-Mittelwerte der Parameter über die Messphasen hinweg graphisch dargestellt - jeweils für die Baseline - und die Follow-upUntersuchung. Zusätzlich wurde das Diagramm durch den Graphen der Kontrollgruppe (der Baseline-Untersuchung) ergänzt, um einen Vergleich mit gesunden Probandinnen zu ermöglichen. Mithilfe des abhängigen t-Tests bzw. mithilfe des Wilcoxon-Vorzeichen-Rang-Tests bei nicht normalverteilten Parametern wurde anschließend auf Unterschiede zwischen Ruhe 1 Baseline und Ruhe 1 Follow-up getestet, wodurch die kardiovaskuläre Ruheaktivierung vor einem Einfluss durch die Stressphasen verglichen werden kann. Außerdem wurden mit jeweils einer 2 x 2 ANOVA für Messwiederholungen die Reaktionen auf die beiden Stressoren (Rechnen bzw. Babyschreien im Vergleich zu Ruhe 1) und der Einfluss des Messzeitpunkts auf diese betrachtet. Zwei weitere 2 x 2 ANOVAs für Messwiederholungen erfolgten, um die Recovery (Rechnen bzw. Babyschreien gegenüber Entspannungsmusik) zu den beiden Messzeitpunkten zu untersuchen. Bei all diesen Berechnungen bzgl. der TFM-Daten wurde sich auf die in Kapitel 3.2.2.3 festgelegten Parameter konzentriert. Für eine Sensitivitätsanalyse mit der Frage nach einem möglichen Einfluss durch Änderungen in der Betablockermedikation sei auf den Anhang verwiesen.

Um die psychologischen Effekte der Therapie zu ermitteln, wurde die Therapiedauer allgemein sowie speziell die Therapiedauer auf der Station 9 des Asklepios Fachklinikums Göttingen (in Tagen) mit den Veränderungen des Stressempfindens und der psychometrischen Fragebögen (von Baseline zu Follow-up) korreliert. Hierbei ist für die Einordnung der Ergebnisse entscheidend, ob Patientinnen mit einer überdurchschnittlich langen Behandlungsdauer bestimmte Merkmale aufweisen (wie beispielsweise eine besonders hohe Krankheitsschwere), aufgrund derer sie eine längere therapeutische Behandlung in Anspruch nehmen mussten. Daher wurden Korrelationen zwischen der Therapiedauer und dem Stressempfinden während der Baseline-Messung bzw. der zwischen Therapiedauer und den 
psychometrischen Fragebögen zum Zeitpunkt Baseline berechnet. Außerdem erfolgte eine multivariate Regression, um den Zusammenhang zwischen der Behandlungsdauer und den Veränderungen des Stressempfindens bzw. der psychometrischen Fragebögen von Baseline zu Follow-up bei gleichzeitiger Adjustierung der jeweiligen Baseline-Werte zu evaluieren.

Zuletzt wurden für die $\Delta \mathrm{R}$-Scores mit verschiedenen Variablen (Differenz des Stressempfindens und der psychometrischen Fragebögen von Baseline zu Follow-up, Therapiedauer allgemein und speziell nur auf der Station 9 in Tagen) Korrelationen berechnet. So sollen mögliche Einflussfaktoren auf die Reagibilität der Patientinnen aufgedeckt werden.

Als Signifikanzkriterium wurde für alle Analysen $p<0,05$ festgelegt, wobei das Signifikanzniveau in den drei Stufen $p<0,05, p<0,01$ und $p<0,001$ angegeben wird. Da die Hypothesen gerichtet formuliert sind, erfolgte i. d. R. eine einseitige Signifikanzprüfung unter Angabe des einseitigen $p$-Werts. Für die Post-hoc-Testung bei ANOVA-Analysen wurde eine Bonferroni-Korrektur des alpha-Niveaus vorgenommen. In den Kapiteln 4.4 und 4.5 erfolgte die Berechnung multipler Korrelationen im Zuge einer rein explorativen Zielsetzung. Hierbei wurde auf eine Bonferroni-Korrektur des alpha-Niveaus zugunsten einer besseren Detektion von Effekten grundsätzlich verzichtet. Ein signifikanter Nachweis ist an dieser Stelle folglich nicht möglich. Für alle Korrelationsberechnungen wurde aufgrund der kleinen Stichprobe und der Verletzung der Normalverteilung bei einigen Zielvariablen durchgängig der Korrelationskoeffizient Spearmans Rho verwendet.

\subsubsection{Recoveryscores und $\Delta \mathrm{R}$-Scores}

Bornschein beschreibt eine höhere Stressreagibilität der Kontrollgruppe gegenüber den PTBS-Patientinnen während der Messung (Bornschein 2014). Eine höhere Reagibilität im Follow-up könnte somit eine Normalisierung des ANS widerspiegeln. Die Stressreagibilität steht für die Flexibilität, mit der das ANS auf Stress reagiert und sich von diesem wieder erholt. Als quantitatives Maß für die Reagibilität soll der maximale Ausschlag von Ruhe/Entspannung zu Stress (Rechnen oder Babyschreien) herangezogen werden. Um diesen maximalen Ausschlag zu erfassen sind zwei Möglichkeiten denkbar: es kann die Reactivity (Vergleich Ruhe 1 und Stressphasen) oder aber die Recovery (Vergleich Musik und Stressphasen) betrachtet werden. Es stellt sich somit die Frage, ob eher die Phase Ruhe 1 oder die Phase Musik mit der geringsten kardiovaskulären Aktivierung einhergeht und damit den besseren Bezugspunkt für den maximalen Ausschlag bildet. Daher werden in Kapitel 4.2.4 die Phasen Ruhe 1 und Musik verglichen. Es soll an dieser Stelle vorweggenommen werden, dass die Phase Entspannungsmusik mit der geringsten kardiovaskulären Aktivierung einhergeht. Die Recovery erscheint somit geeigneter als die Reactivity, die maximale Reagibilität abzubilden. Die Recovery wird somit im Folgenden als Maß für die Reagibilität verwendet.

Zur Quantifizierung der Recovery und damit der Reagibilität wurden für jeden Parameter Recoveryscores für die beiden Stressoren Rechnen und Babyschreien gebildet, wobei jeweils 
die beiden Messzeitpunkte Baseline und Follow-up getrennt betrachtet wurden. So ist der

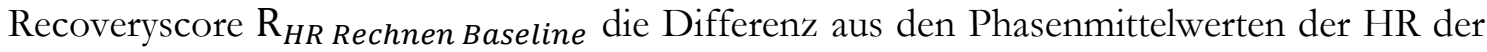
Phasen Rechnen und Musik zum Zeitpunkt Baseline. Um die Veränderung der Recovery von Baseline zu Follow-up ebenfalls abzubilden, wurden zudem für jeden Parameter zwei $\Delta \mathrm{R}-$ Scores der Recovery berechnet - jeweils einer für die Stressoren Rechnen und Babyschreien. Im Folgenden werden beispielhaft die $\Delta \mathrm{R}$-Scores der Recovery anhand der Herzrate erklärt, das Vorgehen gilt jedoch auch für die anderen vier Parameter sBP, TPRI, PEP und BRS.

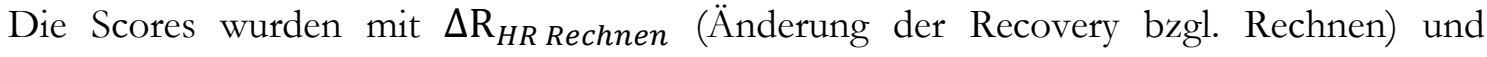
$\Delta \mathrm{R}_{H R \text { Babysch. }}$ (Änderung der Recovery bzgl. Babyschreien) benannt und nach der in Abbildung 2 aufgeführten Formel berechnet. Für die Berechnung von $\Delta R_{H R}$ Rechnen wurde die Differenz aus dem Recoveryscore $R_{H R \text { Rechnen Baseline }}$ und dem Recoveryscore

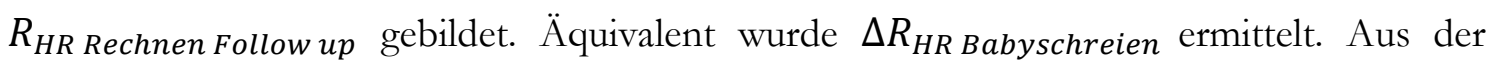
Formel geht außerdem hervor wie die Recoveryscores jeweils berechnet wurden. $H R_{\text {Rechnen Baseline }}$ steht dabei beispielhaft für den Phasenmittelwert der Herzrate in der Phase Rechnen bei der Baseline-Untersuchung. Die Recoveryscores sollen in Kapitel 4.5.1 genauer betrachtet werden. Hierbei soll das Vorzeichen der Recoveryscores berücksichtigt werden, während bei der Berechnung von $\Delta \mathrm{R}$ lediglich der Betrag der Recoveryscores einging. Das Vorzeichen der Recoveryscores zeigt an, in welche Richtung die Stressreaktion von der Phase Musik abwich, ob beispielsweise die Herzrate beim Rechnen gegenüber der Phase Musik höher oder aber niedriger lag. Bei den $\Delta \mathrm{R}$-Scores sollten dagegen lediglich die Unterschiede in der Reagibilität/das Ausmaß der Recovery abgebildet werden. In welche Richtung die Reaktionen abliefen, soll hierbei für eine bessere Beurteilbarkeit der $\Delta \mathrm{R}$-Scores vernachlässigt werden, da angenommen wird, dass die Veränderungen zu beiden Messzeitpunkten in dieselbe Richtung erfolgten, jedoch je nach untersuchter Variable unterschiedliche Vorzeichen haben können. Die $\Delta \mathrm{R}$-Scores ergeben sich somit aus der Differenz (Baseline Follow-up) der Beträge der jeweiligen Recoveryscores für Rechnen bzw. Babyschreien.

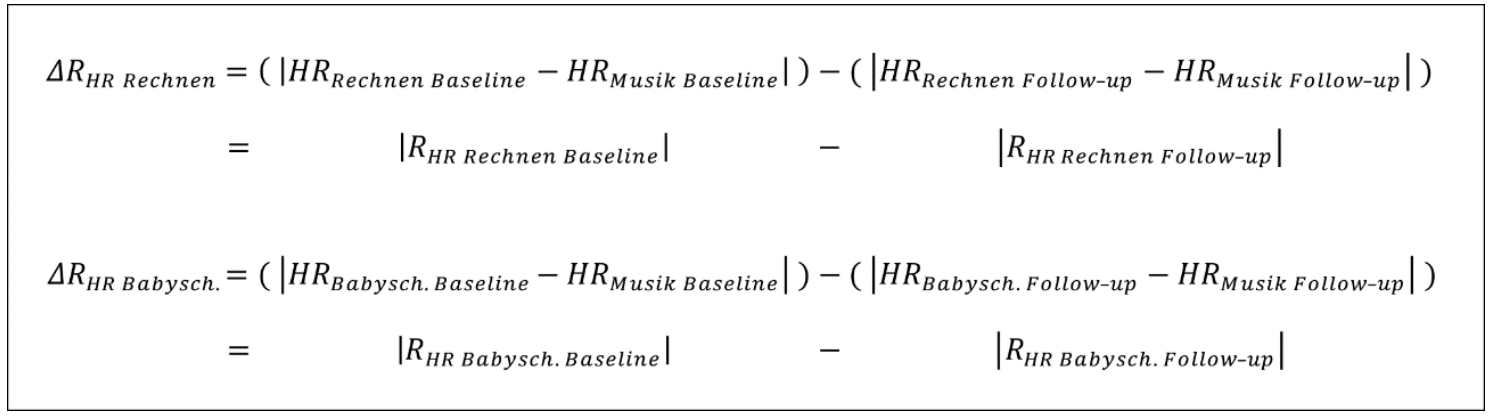

Abbildung 2: Formel zur Berechnung der $\Delta$ R-Scores am Beispiel Herzrate. HR steht hierbei jeweils für den Mittelwert der Herzrate der genannten Phase. R bezeichnet die Recoveryscores. Die Recoveryscores bilden sich jeweils aus der Differenz der Mittelwerte zweier Phasen (HR Rechnen bzw. Babyschreien - HR Musik) von einem Messzeitpunkt (Baseline oder Follow-up). $\Delta$ R berechnet sich dann aus den beiden Recoveryscores von Rechnen bzw. Babyschreien (jeweils Baseline - Followup). Die Striche kennzeichnen, dass ein Wert nur als Betrag in die Rechnung eingeht. 


\section{$4 \quad$ Ergebnisse}

\subsection{Beschreibung des Kollektivs}

\subsubsection{Vollständigkeit der Daten}

Die vorliegende Arbeit konzentriert sich auf die Betrachtung der Patientinnen, die an beiden Untersuchungen (Baseline und Follow-up) teilnahmen. Die folgende Beschreibung des Kollektivs bezieht sich somit nur auf das Follow-up-Kollektiv, das 26 Patientinnen umfasste. Patientinnen, die lediglich die Baseline-Messung absolvierten, werden nicht berücksichtigt. Die Repräsentativität der eingeschlossenen Patientinnen für die Ausgangskohorte wird in Kapitel 4.2.2 untersucht.

Tabelle 1 gibt einen Überblick über die Vollständigkeit der Daten sowie über die Konsequenzen, die sich aus dem Fehlen bestimmter Daten ergaben.

Tabelle 1: Übersicht zu den fehlenden Daten und den davon betroffenen Analysen

\begin{tabular}{|c|c|c|c|c|}
\hline \multicolumn{2}{|c|}{ Fehlende Daten } & $\begin{array}{l}\text { Base- } \\
\text { line }\end{array}$ & $\begin{array}{l}\text { Follow- } \\
\text { up }\end{array}$ & $\begin{array}{l}\text { Ausschluss aus } \\
\text { folgenden } \\
\text { Berechnungen }\end{array}$ \\
\hline \multirow{4}{*}{$\begin{array}{l}\text { TFM- } \\
\text { Daten }\end{array}$} & $\begin{array}{l}\text { Ausschluss der TFM- } \\
\text { Daten einer Patientin }\end{array}$ & 1 & 0 & $\begin{array}{c}\text { Vergleich der } \\
\text { TFM-Daten von Baseline } \\
\text { und Follow-up }\end{array}$ \\
\hline & $\begin{array}{l}\text { Abbruch der } \\
\text { Messung }\end{array}$ & 1 & 0 & $\begin{array}{l}\text { Vergleich der TFM-Daten } \\
\text { von Baseline und Follow- } \\
\text { up (nur bei Berechnungen } \\
\text { mit der Phase Musik) }\end{array}$ \\
\hline & $\begin{array}{l}\text { Störungen der IKG- } \\
\text { Messung: Fehlen des } \\
\text { TPRI in den } \\
\text { Phasen Babyschreien } \\
\text { und Musik } \\
\end{array}$ & 0 & 1 & $\begin{array}{l}\text { Alle Berechnungen mit } \\
\text { dem TPRI aus den Phasen } \\
\text { Babyschreien und Musik } \\
\text { der Follow-up-Messung }\end{array}$ \\
\hline & $\begin{array}{l}\text { Fehlende Berechnung } \\
\text { des PEP-Werts }\end{array}$ & 0 & 3 & $\begin{array}{c}\text { Alle Berechnungen mit } \\
\text { der PEP aus der Follow- } \\
\text { up-Messung }\end{array}$ \\
\hline \multirow{2}{*}{$\begin{array}{l}\text { Psycho- } \\
\text { metrische } \\
\text { Frage- } \\
\text { bögen }\end{array}$} & $\begin{array}{l}\text { Fehlen von allen } \\
\text { psychometrischen } \\
\text { Fragebögen }\end{array}$ & 2 & 0 & $\begin{array}{l}\text { Vergleich der psycho- } \\
\text { metrischen Fragebögen } \\
\text { von Baseline und } \\
\text { Follow-up }\end{array}$ \\
\hline & Fehlen nur der IES-R & 2 & 1 & $\begin{array}{l}\text { Alle Berechnungen mit } \\
\text { der IES-R der Baseline- } \\
\text { Messung bzw. alle } \\
\text { Berechnungen mit der } \\
\text { IES-R allgemein }\end{array}$ \\
\hline $\begin{array}{l}\text { Subjektives } \\
\text { Stress- } \\
\text { empfinden }\end{array}$ & $\begin{array}{l}\text { Fehlen der Angaben } \\
\text { zu einer Messung }\end{array}$ & 1 & 0 & $\begin{array}{c}\text { Vergleich des } \\
\text { Stressempfindens von } \\
\text { Baseline und Follow-up }\end{array}$ \\
\hline
\end{tabular}


Von den 26 Patientinnen wurde eine bei der Baseline-Untersuchung aufgrund von höhergradigen Überleitungsstörungen im EKG ausgeschlossen (Albrecht 2013). Bei dem Followup zeigte sich das EKG dieser Patientin jedoch unauffällig, sodass hier ein Ausschluss nicht notwendig war. Bei Berechnungen, bei denen ein Vergleich zu den TFM-Daten in der Baseline-Untersuchung erfolgte, war die Patientin folglich ausgeschlossen; bei allen anderen Analysen wurde sie einbezogen.

Die Baseline-Untersuchung wurde von einer Patientin vorzeitig beendet, sodass hier die Aufzeichnungen nach der Phase Babyschreien fehlen. Die gleiche Patientin brach auch im Follow-up die Phase Babyschreien ab. Im Gegensatz zur Baseline-Untersuchung entschied sich die Patientin im Follow-up jedoch die Messung nach der Phase Babyschreien fortzuführen und wie üblich mit Entspannungsmusik abzuschließen. Obwohl der Stressor Babyschreien statt der üblichen fünf Minuten nur etwa drei Minuten wirkte, ist aufgrund der sehr emotionalen Reaktion der Patientin (starkes Weinen) davon auszugehen, dass der Stressor ausreichend gesetzt war. Die Patientin wurde somit nur für Berechnungen ausgeschlossen, bei denen die Baseline-TFM-Daten der Phase Entspannungsmusik eingingen. Alle anderen Rechnungen (mit den vor dem Abbruch aufgezeichneten Phasen in der Baseline-Untersuchung) wurden ohne Ausschluss dieser Patientin durchgeführt. Auch die gesamten Followup-Daten sowie die psychometrischen Fragebögen und ihr Stressempfinden wurden ausgewertet.

Im Follow-up trat bei einer Patientin, vermutlich durch Elektrodenablösung, eine Störung der IKG-Messung auf, sodass hier die IKG-Parameter (u. a. der TPRI) für die Phasen Babyschreien und Musik fehlen. Bei drei weiteren Patientinnen konnte der PEP-Wert von dem System nicht berechnet werden, ihre restlichen TFM-Daten sind jedoch davon unberührt.

Die allgemeinen Fragebögen zu soziodemographischen Daten und Therapieverlauf sowie die psychometrischen Fragebögen füllten alle Patientinnen im Follow-up aus. Bei der Baseline-Untersuchung fehlen dagegen die Daten zu den psychometrischen Fragebögen bei zwei Patientinnen komplett, ein Vergleich mit dem Follow-up war bei diesen Patientinnen daher nicht möglich. Zwei Patientinnen ließen zudem bei der Baseline-Untersuchung einzig die IES-R aus, eine der beiden verweigerte auch im Follow-up das Ausfüllen der IES-R.

Bezüglich des subjektiven Stressempfindens fehlen nur von einer Patientin die Angaben für die Baseline-Messung, die Daten des Follow-ups sind hier vollständig. Aufgrund von Vorerkrankungen oder Medikamenteneinnahme musste keine Patientin aus der Auswertung ausgeschlossen werden.

Insgesamt sind 21 TFM-Datensätze vollständig. Zwei Patientinnen fehlen bei Vergleichen zwischen Baseline und Follow-up, vier Patientinnen bei Berechnungen mit bestimmten Parametern (PEP, TPRI). Von 24 Patientinnen liegen die psychometrischen Fragebögen sowohl für den Zeitpunkt Baseline als auch für das Follow-up ausgefüllt vor (ausgenommen der IES-R, die nur bei 22 Patientinnen vollständig ist). Alle 26 Patientinnen gaben ihr Stressempfinden im Follow-up an, bei der Baseline-Untersuchung waren es 25. 


\subsubsection{Soziodemographische Daten}

Bei der Follow-up-Untersuchung war die älteste Patientin 59 und die jüngste 28 Jahre alt, der Altersdurchschnitt lag bei 45,7 (SD = 8,8) Jahren. Die Patientinnen hatten im Durchschnitt einen Body Mass Index von 28,6 (SD = 6,2) kg/m². Die Patientinnen, die rauchten $(38,5 \%$ des Follow-up-Kollektivs), kamen im Mittel auf eine Anzahl von 23,1 ( $S D=25,0)$ packyears. Die Anzahl an packyears schwankte somit unter den Raucherinnen erheblich. 73,1\% der Patientinnen gaben an gar keinen Alkohol zu trinken. Eine Patientin ließ die Frage nach dem Alkoholkonsum offen. Die restlichen sechs Patientinnen konsumierten im Schnitt 2,8 (SD $=1,8)$ drinks per week. Das Maximum lag hier bei 5,0 drinks per week. Ein Drink wird dabei entweder als 0,25 Liter Bier, als 0,1 Liter Wein oder als $2 \mathrm{cl}$ Schnaps definiert. Fast jede zweite Patientin ging zum Zeitpunkt des Follow-ups keiner sportlichen Betätigung nach (s. Tab. 2). Zehn Patientinnen gaben ihre sportliche Betätigung pro Woche mit unter zwei Stunden an. Lediglich drei Patientinnen trieben über zwei Stunden Sport pro Woche. Eine Patientin machte zu dieser Frage keine Angaben.

Tabelle 2: Sportliche Betätigung des Kollektivs zum Zeitpunkt Follow-up $(N=25)$

\begin{tabular}{|l|c|c|}
\hline \multicolumn{1}{|c|}{ Sportliche Betätigung pro Woche } & $\begin{array}{c}\text { Häufigkeit } \\
\text { (absolut) }\end{array}$ & $\begin{array}{c}\text { Häufigkeit } \\
\text { (prozentual) }\end{array}$ \\
\hline Kein Sport & 12 & 48,0 \\
\hline Unter einer halben Stunde Sport pro Woche & 4 & 16,0 \\
\hline Eine halbe bis zwei Stunden Sport pro Woche & 6 & 24,0 \\
\hline Zwei bis fünf Stunden Sport pro Woche & 2 & 8,0 \\
\hline Fünf bis zehn Stunden Sport pro Woche & 1 & 4,0 \\
\hline
\end{tabular}

Tabelle 3 zeigt die Familien- und Partnerschaftsverhältnisse des Follow-up-Kollektivs. Die Hälfte der Patientinnen befand sich zum Zeitpunkt des Follow-ups in einer festen, in einem Haushalt lebenden Partnerschaft. 11,5 \% hatten zwar einen festen Partner, lebten jedoch nicht mit ihm zusammen. 38,5 \% waren in keiner festen Beziehung. Bei der Frage nach dem Familienstand gaben 34,5\% an ledig zu sein, 30,8 \% waren in erster Ehe verheiratet, 23,1 \% geschieden und 11,5\% befanden sich in ihrer mindestens zweiten Ehe. 61,5\% der Patientinnen waren Mütter und hatten im Durchschnitt 1,8 $(S D=1,0)$ Kinder. Dabei hatte eine Patientin einen sechs Monate alten Sohn, die restlichen Kinder waren deutlich älter. Einen Überblick über die Schulabschlüsse, Ausbildung und Erwerbstätigkeit des Follow-upKollektivs gibt Tabelle 4. Alle Patientinnen besaßen einen Schulabschluss. Mit 42,3 \% überwog dabei das Abitur. 19,2\% waren auf die Hauptschule gegangen, 30,8 \% auf die Realschule und die restlichen 7,7\% auf eine Fachoberschule. Keine der Patientinnen befand sich zum Zeitpunkt der Untersuchung in einer Ausbildung. 11,5\% wiesen keinen Ausbildungsabschluss auf. Bei den restlichen Patientinnen zeigte sich ein breit gefächertes Bild an Ausbildung, von der Lehre bis zur Hochschule. Fragte man die Patientinnen jedoch nach ihrer gegenwärtigen Erwerbstätigkeit, war der Großteil gar nicht erwerbstätig (73,1 \%). Nur drei der 26 Patientinnen arbeiteten in Vollzeit. Drei weitere Patientinnen arbeiteten unter 15 Stunden pro Woche und eine Patientin zwischen 15 und 35 Stunden. 
Tabelle 3: Familie und Partnerschaft des Kollektivs zum Zeitpunkt Follow-up $(N=26)$

\begin{tabular}{|c|l|c|c|}
\hline \multicolumn{2}{|c|}{ Familie und Partnerschaft } & $\begin{array}{c}\text { Häufigkeit } \\
\text { (absolut) }\end{array}$ & $\begin{array}{c}\text { Häufigkeit } \\
\text { (prozentual) }\end{array}$ \\
\hline \multirow{3}{*}{$\begin{array}{c}\text { Partner- } \\
\text { schaft }\end{array}$} & Nein, keine Partnerschaft & 10 & 38,5 \\
\cline { 2 - 4 } & Ja, feste Partnerschaft - nicht zusammenlebend & 3 & 11,5 \\
\cline { 2 - 4 } & Ja, feste Partnerschaft - zusammenlebend & 13 & 50,0 \\
\hline \multirow{4}{*}{$\begin{array}{c}\text { Familien- } \\
\text { stand }\end{array}$} & ledig & 9 & 34,6 \\
\cline { 2 - 4 } & Verheiratet in erster Ehe & 8 & 30,8 \\
\cline { 2 - 4 } & geschieden & 6 & 23,1 \\
\cline { 2 - 4 } & Geschieden und wieder verheiratet & 3 & 11,5 \\
\hline \multirow{3}{*}{ Kinder } & Keine Kinder & 10 & 38,5 \\
\cline { 2 - 4 } & Ein Kind & 8 & 30,8 \\
\cline { 2 - 4 } & Zwei Kinder & 3 & 15,4 \\
\cline { 2 - 4 } & Drei Kinder & 1 & 3,5 \\
\cline { 2 - 4 } & Vier Kinder & & \\
\hline
\end{tabular}

Tabelle 4: Ausbildung und Erwerbstätigkeit des Kollektivs zum Zeitpunkt Follow-up ( $N=26)$

\begin{tabular}{|c|c|c|c|}
\hline \multicolumn{2}{|c|}{ Ausbildung und Erwerbstätigkeit } & $\begin{array}{l}\text { Häufigkeit } \\
\text { (absolut) }\end{array}$ & $\begin{array}{l}\text { Häufigkeit } \\
\text { (prozentual) }\end{array}$ \\
\hline \multirow{4}{*}{ Schulabschluss } & Hauptschule/Volksschule & 5 & 19,2 \\
\hline & Realschule (Mittlere Reife) & 8 & 30,8 \\
\hline & Fachoberschule & 2 & 7,7 \\
\hline & Gymnasium (Abitur) & 11 & 42,3 \\
\hline \multirow{7}{*}{$\begin{array}{l}\text { Ausbildungs- } \\
\text { abschluss }\end{array}$} & Kein Ausbildungsabschluss & 3 & 11,5 \\
\hline & Beruflich-betriebliche Ausbildung (Lehre) & 7 & 26,9 \\
\hline & $\begin{array}{l}\text { Beruflich-schulische Ausbildung } \\
\text { (Berufsfach-/Handelsschule) }\end{array}$ & 6 & 23,1 \\
\hline & $\begin{array}{l}\text { Fach-/Meister-/Technikerschule } \\
\text { /Berufs-/Fachakademie }\end{array}$ & 3 & 11,5 \\
\hline & Fachhochschule & 2 & 7,7 \\
\hline & Hochschule & 4 & 15,4 \\
\hline & Anderer Ausbildungsabschluss & 1 & 3,8 \\
\hline \multirow{4}{*}{ Erwerbstätigkeit } & Nicht erwerbstätig & 19 & 73,1 \\
\hline & $<15$ Stunden pro Woche & 3 & 11,5 \\
\hline & 15 - 35 Stunden/Woche & 1 & 3,8 \\
\hline & $>35$ Stunden/Woche (Vollzeit) & 3 & 11,5 \\
\hline
\end{tabular}

\subsubsection{Psychiatrische Diagnosen und Suchtverhalten}

Die folgenden Daten wurden 2010 gemäß der Krankenakten auf der Station 9 des Asklepios Fachklinikums Göttingen erhoben. Bei allen 26 Patientinnen wurde eine Posttraumatische Belastungsstörung nach ICD-10 F43.1 diagnostiziert. Dabei lag bei sieben Patientinnen (26,9 \%) eine komplexe Posttraumatische Belastungsstörung nach Herman vor. Eine Chronifizierung der PTBS wurde bei neun Patientinnen explizit in den Krankenakten vermerkt. Die Krankheitsverläufe der Patientinnen geben jedoch Grund zu der Annahme, dass bei dem Großteil der Patientinnen eine chronische PTBS vorlag. 
Ursächlich für die Traumatisierung war überwiegend ein isoliertes Typ 2-Trauma (69,2 \%); ein isoliertes Typ 1-Trauma als Grund für die PTBS war dagegen seltener (11,5\%). Weitere 11,5 \% hatten sowohl ein Typ 1- als auch ein Typ 2-Trauma erlitten. Bei den restlichen zwei Patientinnen konnte aufgrund der vorliegenden Aufzeichnungen keine eindeutige Zuordnung zu einem Traumatyp vorgenommen werden. Für den Großteil der Patientinnen $(80,8 \%)$ sind traumatisierende Ereignisse in der Kindheit dokumentiert, häufig in Form von sexueller und körperlicher Gewalt sowie Vernachlässigung und emotionaler Misshandlung im familiären Umfeld. Die traumatisierenden Zustände hielten hierbei oft über Jahre an.

Entsprechend dem Konzept eines externalisierenden und internalisierenden Typus können sowohl die Borderline-Persönlichkeitsstörung als auch dissoziative Störungen in das Feld der komplexen Traumafolgestörungen eingeordnet werden. Zusätzlich zu der PTBS-Diagnose wurde bei zwölf Patientinnen eine Persönlichkeitsstörung festgestellt. Davon wiesen zehn Patientinnen (38,5 \%) eine emotional instabile Persönlichkeitsstörung des Borderline-Typs (nach ICD-10: F60.31) auf, eine dieser Patientinnen zeigte zusätzlich Anteile einer schizoiden Persönlichkeitsstörung (F60.1). Außerdem hatte eine Patientin eine ängstlich vermeidende (F60.6) und eine weitere Patientin eine abhängige Persönlichkeitsstörung (F60.7). Vierzehn Patientinnen litten zudem unter einer dissoziativen Amnesie (nach ICD-10: F44.0). Zehn Patientinnen hatten zusätzlich zur dissoziativen Amnesie bzw. unabhängig davon ein Depersonalisations- und Derealisationssyndrom (F48.1). Außerdem waren dissoziative Identitätsstörungen (F44.81), DDNOS (dissociative disorder not otherwise specified, F44.9), dissoziatives Fugue (F44.1) und dissoziative Sensibilitäts- und Empfindungsstörungen (F44.6) bei den Patientinnen zu finden. Insgesamt waren 17 Patientinnen (65,4 \%) von den verschiedenen Formen der dissoziativen Störung beeinträchtigt. Auch wenn eine einheitliche Klassifikation sowie Diagnosekriterien für die komplexen Traumafolgestörungen noch ausstehen, sind dennoch wichtige Kennzeichen dieses Störungskomplexes erfüllt. Die Kohorte kann somit den komplexen Traumafolgestörungen zugeordnet werden.

Eine häufige Komorbidität der PTBS ist die Depression. Damit übereinstimmend war bei allen Patientinnen eine rezidivierende depressive Störung bekannt, die den verschiedenen Unterklassen der ICD-10-Kategorie F33.- zugeordnet werden konnte. Die depressive Symptomlast zum Zeitpunkt Follow-up wurde mit dem BDI genauer bestimmt. Neun Patientinnen $(34,6 \%$ ) hatten Somatisierungsstörungen (F45.0) bzw. somatoforme Schmerzstörungen (F45.4). Bei ebenfalls neun Patientinnen lag eine Angststörung vor, wobei sowohl die Panikstörung nach F41.0 als auch phobische Störungen (F40.-) wie die Agoraphobie und die soziale Phobie vorkamen. Selbstverletzendes Verhalten zeigten elf Patientinnen (42,3\%), während bei fünf Patientinnen ein Suizidversuch in der Vergangenheit dokumentiert war. Vier Patientinnen litten unter einer Essstörung wie beispielsweise Bulimia nervosa. Eine Patientin war an einer Zwangsstörung erkrankt (F42.1). Ein Alkoholabusus war bei sechs (23,1 \%), ein Medikamentenabusus bei vier Patientinnen (15,4\%) dokumentiert. Zu dem Medikamentenabusus wurde ein schädigender Konsum von THC gerechnet. Ansonsten wurden überwiegend Benzodiazepine und Analgetika eingenommen. Insgesamt galten fünf 
Patientinnen aufgrund ihres Medikamente- oder Alkoholabusus als suchtkrank. Elf Patientinnen konsumierten weder Nikotin noch in Übermaßen Alkohol oder Medikamente. Insgesamt kann die Kohorte hinsichtlich Komorbiditäten, Suchtverhalten und Suizidalität bzw. selbstverletzendem Verhalten als typisch für das Erkrankungsbild der komplexen Traumafolgestörungen gewertet werden.

\subsubsection{Somatische Erkrankungen}

Neben den psychiatrischen Diagnosen hatten die Patientinnen auch eine Vielzahl an somatischen Erkrankungen. Diese sollen aufgrund ihres potenziellen Einflusses auf die Ergebnisse der TFM-Messung genauer betrachtet werden. Die Tabelle 5 gibt einen Überblick über die somatischen Erkrankungen der Patientinnen zum Zeitpunkt des Follow-ups. Die hierzu verwendeten Daten wurden 2010 den Akten der Station 9 des Asklepios Fachklinikums Göttingen entnommen und mit den Angaben, die die Patientinnen 2013 in den allgemeinen Fragebögen zu ihren Vorerkrankungen machten, ergänzt und aktualisiert. Besonders häufig waren Schilddrüsenerkrankungen sowie die arterielle Hypertonie in dem Kollektiv, aber auch Atemwegserkrankungen und Bandscheibenprobleme bzw. chronische Rückenschmerzen waren immerhin bei jeder fünften Patientin zu finden. Da bei keiner Patientin Erkrankungen wie Herzrhythmusstörungen oder eine Herzinsuffizienz bekannt waren, können die Einflüsse der Krankheiten auf die TFM-Messung als gering angenommen werden. Keine Patientin musste daher wegen einer Vorerkrankung ausgeschlossen werden.

Tabelle 5: Die somatischen Erkrankungen des Kollektivs zum Zeitpunkt Follow-up ( $N=26)$

\begin{tabular}{|l|c|c|}
\hline \multicolumn{1}{|c|}{ Somatische Erkrankungen } & $\begin{array}{c}\text { Häufigkeit } \\
\text { (absolut) }\end{array}$ & $\begin{array}{c}\text { Häufigkeit } \\
\text { (prozentual) }\end{array}$ \\
\hline Keine somatische Erkrankungen & 5 & 19,2 \\
\hline Schilddrüsenerkrankungen & 9 & 34,6 \\
\hline Arterielle Hypertonie & 8 & 30,8 \\
\hline Atemwegserkrankungen & 5 & 19,2 \\
\hline Bandscheibenprobleme, chronische Rückenschmerzen & 5 & 19,2 \\
\hline Gastrointestinale Erkrankungen & 4 & 15,4 \\
\hline Migräne & 2 & 7,7 \\
\hline Gefäßerkrankungen & 2 & 7,7 \\
\hline Dermatologische Erkrankungen & 2 & 7,7 \\
\hline Tumorerkrankung & 1 & 3,8 \\
\hline Lebererkrankung & 1 & 3,8 \\
\hline Rheumatische Erkrankung & 1 & 3,8 \\
\hline Koronare Herzkrankheit & 1 & 3,8 \\
\hline Restless-Legs-Syndrom & 1 & 3,8 \\
\hline
\end{tabular}




\subsubsection{Medikamente}

Die Daten zu den Medikamenten (s. Tab. 6) beruhen auf den Angaben der Patientinnen. Von den 26 Patientinnen nahmen zwei zum Zeitpunkt der Follow-up-Untersuchung gar keine Medikamente ein. Die am meisten verordnete Medikamentenklasse waren die Antidepressiva. Insgesamt 76,9 \% der Patientinnen bekamen zum Follow-up-Zeitpunkt Antidepressiva verschrieben, dabei waren SSRI mit 10 Patientinnen die häufigste Klasse. Aber auch SSNRI, trizyklische Antidepressiva und andere Antidepressiva (Bupropion, Agomelatin, Mirtazapin, Tianeptin) wurden eingenommen.

Tabelle 6: Medikation des Kollektivs zum Zeitpunkt Follow-up $(N=26)$. Das Parkinsonmittel war Pramipexol und aufgrund eines Restless-Legs-Syndroms indiziert.

\begin{tabular}{|c|c|c|c|}
\hline & Medikamente & $\begin{array}{c}\text { Häufigkeit } \\
\text { (absolut) }\end{array}$ & $\begin{array}{l}\text { Häufigkeit } \\
\text { (prozentual) }\end{array}$ \\
\hline \multicolumn{2}{|l|}{ Keine Medikamente } & 2 & 7,7 \\
\hline \multicolumn{2}{|l|}{ Neuroleptika } & 6 & 23,1 \\
\hline \multicolumn{2}{|c|}{ Sedativa (Benzodiazepine, Promethazin, Zolpidem) } & 7 & 26,9 \\
\hline \multicolumn{2}{|c|}{ Analgetika (NSAR, Metamizol, Opioide) } & 5 & 19,2 \\
\hline \multirow{5}{*}{ Antidepressiva } & Gesamt & 20 & 76,9 \\
\hline & Trizyklische Antidepressiva & 3 & 11,5 \\
\hline & SSNRI & 6 & 23,1 \\
\hline & SSRI & 10 & 38,5 \\
\hline & Andere Antidepressiva & 5 & 19,2 \\
\hline \multirow{5}{*}{ Antihypertensiva } & Gesamt & 10 & 38,5 \\
\hline & Betablocker & 7 & 26,9 \\
\hline & ACE-Hemmer/AT1-Antagonisten & 5 & 19,2 \\
\hline & Calciumantagonisten & 3 & 11,5 \\
\hline & Sonstige Antihypertensiva & 2 & 7,7 \\
\hline \multirow{10}{*}{$\begin{array}{c}\text { Sonstige } \\
\text { Medikation }\end{array}$} & Gesamt & 11 & 42,3 \\
\hline & Schilddrüsenhormone & 8 & 30,8 \\
\hline & Thyreostatika & 1 & 3,8 \\
\hline & ASS & 3 & 11,5 \\
\hline & Phenprocoumon & 1 & 3,8 \\
\hline & Protonenpumpeninhibitoren & 2 & 7,7 \\
\hline & Anticholinergika & 2 & 7,7 \\
\hline & Antiepileptika & 1 & 3,8 \\
\hline & Parkinsonmittel & 1 & 3,8 \\
\hline & Hormonelle Kontrazeptiva & 2 & 7,7 \\
\hline
\end{tabular}

Des Weiteren waren Antihypertensiva als Medikation häufig (38,5\%). Am meisten wurden hier Betablocker eingenommen, darauf folgten ACE-Hemmer bzw. AT1-Antagonisten, Calciumantagonisten und selten andere Antihypertensiva (Renininhibitoren, Schleifendiuretika). Sechs Patientinnen (23,1\%) bekamen Neuroleptika verschrieben, sieben Patientinnen benötigten, teilweise jedoch nur bei Bedarf, Sedativa (zwei Patientinnen verwendeten Zolpidem als Schlafmittel). Analgetika nahmen fünf der Patientinnen, eine erhielt ein Opioid. 
Bei den restlichen Medikamenten waren Schilddrüsenmedikamente besonders häufig; acht Patientinnen gaben Schilddrüsenhormone oder Thyreostatika als Medikation an. Anticholinergika verdienen aufgrund ihres möglichen Einflusses auf die Messergebnisse besondere Beachtung. Sie wurden jedoch nur von zwei Patientinnen eingenommen (Propiverin, Trospiumchlorid). Allerdings haben natürlich auch die trizyklischen Antidepressiva und einige der Neuroleptika wie Promethazin oder Prothipendyl eine deutlich anticholinerge Wirkung, die zu berücksichtigen ist.

Die Medikamenteneinnahme gehört, wie auch die vielfältigen Komorbiditäten, zur Natur einer Kohorte mit komplexen Traumafolgestörungen. Sie erscheint unter diesen Voraussetzungen nicht als überdurchschnittlich hoch.

\subsubsection{Therapiezeiten zwischen den Messungen}

Mittels des Fragebogens zum Therapieverlauf (s. Anhang, Kap. 8.8) wurden die stationären somatischen Behandlungen sowie die psychotherapeutischen oder psychiatrischen stationären und ambulanten Behandlungen in dem Zeitraum zwischen der Baseline- und der Followup-Messung erhoben. Dabei ist von besonderem Interesse, wie lange die Behandlung auf der Station 9 des Asklepios Fachklinikums Göttingen andauerte. Betrachtet man die Summe der Behandlungstage auf der Station 9 in dem Zeitraum 2009 bis 2013, wird ersichtlich, dass es hier eine sehr breite Streuung gibt (s. Abb. 3). Die Spannbreite reicht von 8 bis 241 Tage.

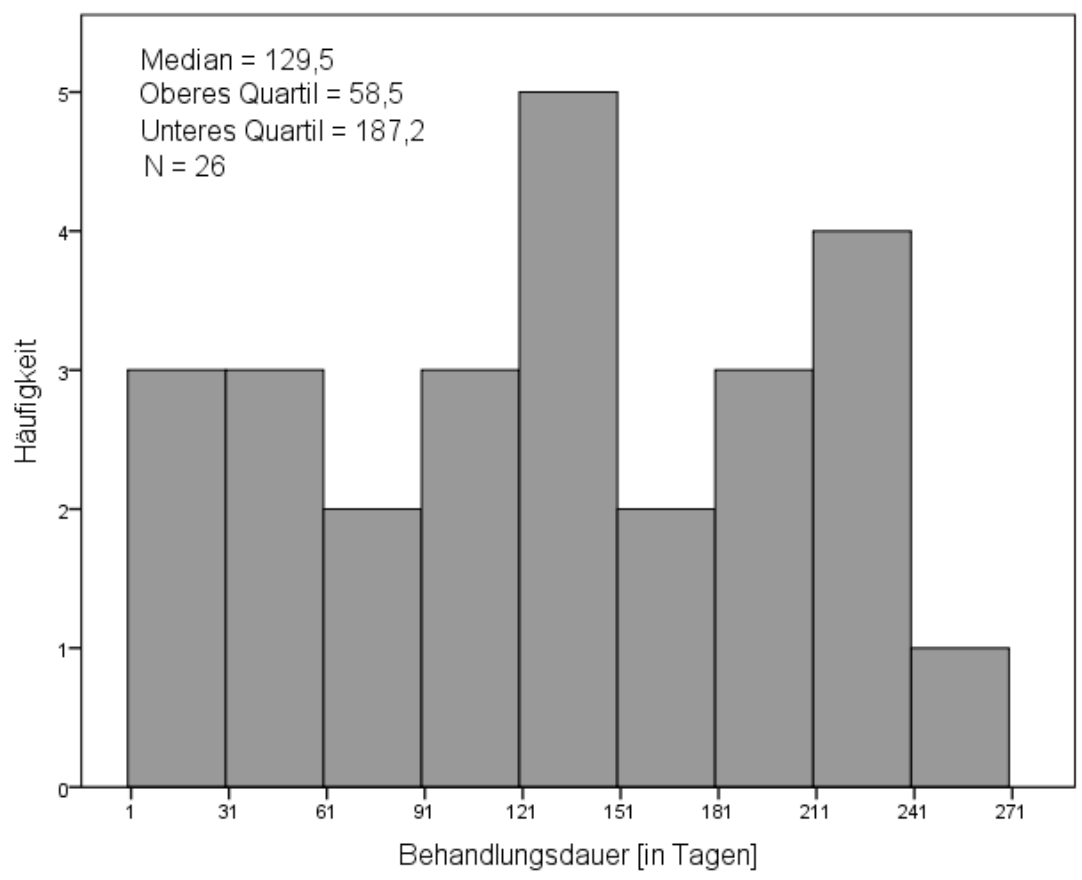

Abbildung 3: Stationäre Psychotherapie der Follow-up-Teilnehmerinnen auf der Station 9 des Asklepios Fachklinikums Göttingen zwischen 2009 und 2013 in Tagen $(N=26)$ 
Die niedrige Behandlungsdauer bei einigen Patientinnen kann dabei auf verschiedene Gründe zurückgeführt werden. Zwei Patientinnen waren nur für die Diagnostikphase auf der Station, in der aber festgestellt wurde, dass die spezifische Therapie auf der Station 9 nicht für sie geeignet war. Andere Patientinnen brachen bereits in einer frühen Phase die Therapie ab. Aber auch bei Patientinnen, die mehrere Therapiezyklen auf der Station 9 absolvierten, kommt es zu sehr unterschiedlichen Therapiedauern, zum einen da die Therapiedauern allgemein individuell sehr divergieren, zum anderen da einige Patientinnen auch in späteren Phasen die Therapie abbrachen. Zuletzt hatten nicht alle Patientinnen bei der Follow-upUntersuchung ihre Therapie vollständig absolviert, teilweise standen noch weitere Therapiezyklen aus. Folglich kann nicht davon ausgegangen werden, dass alle Patientinnen in gleicher Weise von der Therapie auf Station 9 profitierten, die individuellen Therapiedauern müssen berücksichtigt werden.

Die Patientinnen gaben ebenfalls an, wenn sie sich auf einer anderen Station oder in einem anderen Krankenhaus in stationärer psychiatrischer oder psychotherapeutischer Behandlung befunden hatten. Summiert man diese Behandlungstage zu den Aufenthalten auf der Station 9 des Asklepios Fachklinikums Göttingen lässt sich die gesamte Dauer der stationären psychiatrischen bzw. psychotherapeutischen Behandlung der Patientinnen in den Jahren 2009 bis 2013 ablesen (s. Abb. 4). Hier findet sich eine sogar noch größere Streuung. Somit kann auch bezüglich der gesamten stationären psychiatrischen oder psychotherapeutischen Behandlung nicht von einer einheitlichen Therapiedauer der Patientinnen ausgegangen werden.

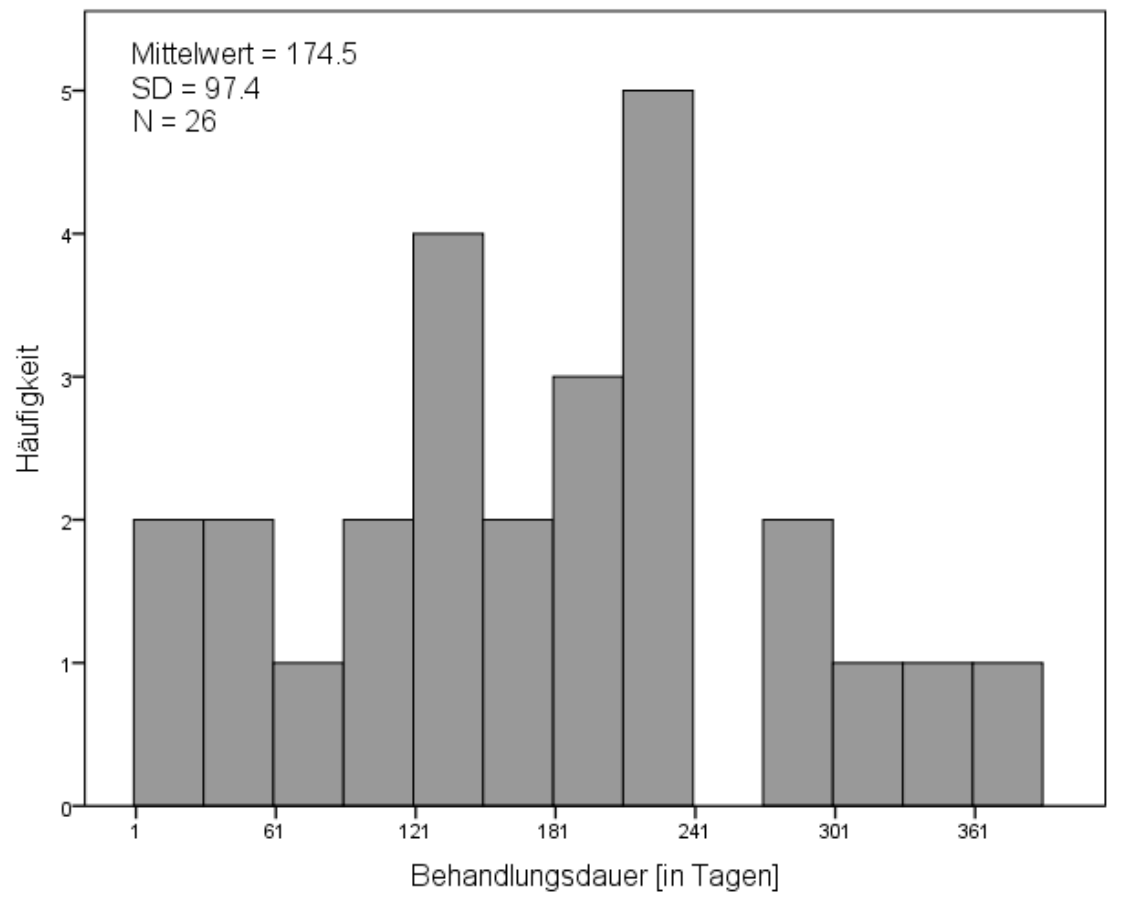

Abbildung 4: Stationäre Therapie der Follow-up-Teilnehmerinnen auf einer psychiatrischen oder psychotherapeutischen Station (inklusive der Station 9 des Asklepios Fachklinikums Göttingen) zwischen 2009 und 2013 in Tagen $(N=26)$ 
Aufgrund der teilstationären Charakteristik wurden Therapien im Zuge einer Tagesklinik gesondert gezählt. Nur vier der Patientinnen waren jedoch in dem Zeitraum in einer Tagesklinik behandelt worden - eine wurde mit 298 Tagen sehr lange tagesklinisch therapiert. Alle diese vier Patientinnen hatten jedoch neben der Tagesklinik auch eine hohe Anzahl an stationären Behandlungstagen. Eine kurze stationäre Behandlungsdauer wurde somit nicht durch einen Tagesklinikaufenthalt ausgeglichen.

Abbildung 5 stellt die ambulanten psychotherapeutischen Behandlungen der Patientinnen in den Jahren 2009 bis 2013 dar. Für eine bessere Vergleichbarkeit wurden die Jahre 2009 und 2013 bei allen Patientinnen komplett mitgezählt - auch wenn diese z. T. nicht im Zeitraum zwischen den beiden Untersuchungen lagen -, sodass sich ein Behandlungszeitraum von maximal fünf Jahren ergibt. 42,3 \% der Patientinnen befanden sich durchgehend während dieser Zeit und damit auch zwischen den beiden Messzeitpunkten in einer ambulanten Therapie. 19,2 \% nahmen keine ambulante Therapie in Anspruch, die restlichen Patientinnen wurden zumindest zeitweise psychotherapeutisch betreut. Ebenfalls 42,3\% der Patientinnen wurden zeitweise oder über den gesamten Zeitraum durch einen ambulanten Neurologen /Psychiater betreut.

Insgesamt kristallisierten sich drei Patientinnen heraus, die sowohl stationär als auch ambulant wenig psychotherapeutische Behandlungen in Anspruch nahmen. Eine Patientin war nur 8 Tage stationär und zwei Jahre ambulant betreut, die zweite Patientin war 32 Tage stationär und ein Jahr ambulant betreut und die dritte Patientin war zwar 61 Tage in stationärer, aber gar nicht in ambulanter Betreuung.

Acht Patientinnen waren aufgrund einer somatischen Erkrankung stationär aufgenommen worden. Eine Patientin befand sich für zehn Tage aufgrund von Blutdruckeinstellung und Herzrhythmusstörungen auf einer kardiologischen Station. Da sich das EKG dieser Patientin jedoch als unauffällig erwies und die Patientin keine diagnostizierte Herzrhythmusstörung als Vorerkrankung angab, wurde die Patientin nicht ausgeschlossen. Eine weitere Patientin hatte einen Eingriff im Herzkatheterlabor, welches ebenfalls keinen Ausschlussgrund darstellt. Weitere längere stationäre Aufenthalte ergaben sich aufgrund einer akuten Hepatitis sowie aufgrund eines Schädelhirntraumas und einer Epilepsie. 


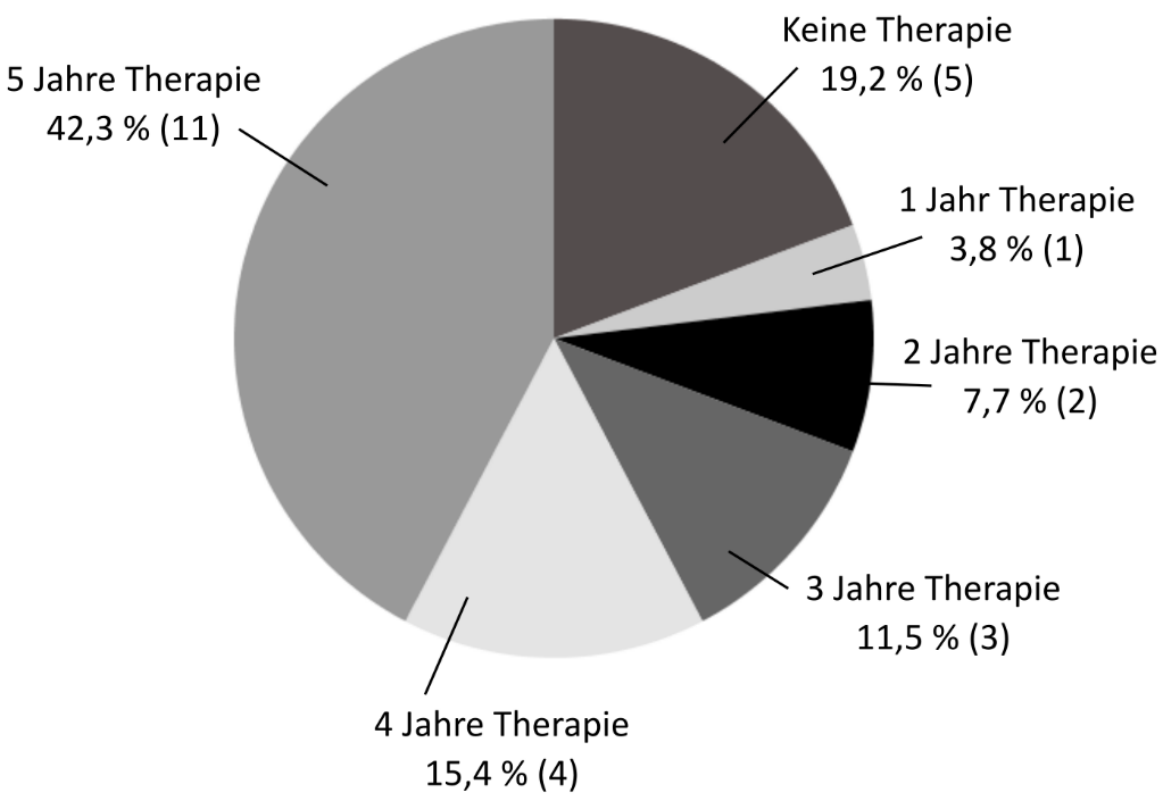

Abbildung 5: Ambulante psychotherapeutische Behandlungen der Follow-up-Teilnehmerinnen ( $N=$ 26) zwischen 2009 und 2013: Angegeben sind die prozentualen Häufigkeiten sowie die absoluten Häufigkeiten in Klammern.

\subsubsection{Psychometrischer Befund}

Für die SCL-90-R wurden jeweils die Summenwerte der neun Skalen sowie die drei globalen Kennwerte berechnet. Hieraus wurden die Mittelwerte und Standardabweichungen für das Kollektiv ermittelt und anhand des deutschen SCL-90-R-Manuals von Franke (2000) in alters- und geschlechterspezifische T-Werte transformiert (s. Tab. 7).

Tabelle 7: Mittelwerte, Standardabweichungen und T-Werte zu den Subskalen und globalen Kennwerten der SCL-90-R des Kollektivs zum Zeitpunkt Follow-up $(N=26)$

\begin{tabular}{|c|l|c|c|c|}
\hline \multicolumn{2}{|l|}{ SCL-90-R } & Mittelwert & SD & T-Werte \\
\hline \multirow{5}{*}{ Subskalen } & Aggressivität/Feindseligkeit & 6,6 & 5,4 & 66 \\
\cline { 2 - 5 } & Ängstlichkeit & 15,5 & 10,5 & 68 \\
\cline { 2 - 5 } & Depressivität & 25,0 & 12,1 & 70 \\
\cline { 2 - 5 } & Paranoides Denken & 8,2 & 6,1 & 64 \\
\cline { 2 - 5 } & Phobische Angst & 9,8 & 7,9 & 71 \\
\cline { 2 - 5 } & Psychotizismus & 10,8 & 8,9 & 67 \\
\cline { 2 - 5 } & Somatisierung & 18,2 & 12,3 & 67 \\
\cline { 2 - 5 } & Unsicherheit im Sozialkontakt & 14,4 & 9,8 & 68 \\
\cline { 2 - 5 } & Zwanghaftigkeit & 16,8 & 8,5 & 71 \\
\hline \multirow{3}{*}{$\begin{array}{c}\text { Globale } \\
\text { Kennwerte }\end{array}$} & GSI & 1,5 & 0,9 & 71 \\
\cline { 2 - 5 } & PST & 59,9 & 19,8 & 66 \\
\cline { 2 - 5 } & PSDI & 2,2 & 0,6 & 65 \\
\hline
\end{tabular}

Alle T-Werte ab 60 gelten in der Regel als erhöht. Um eine grobe Einordnung der T-Werte vorzunehmen schlägt Franke in ihrem Manual eine Faustregel vor (Franke 2000). Hiernach werden T-Werte von 60 bis 64 als „leicht erhöht“, von 65 bis 69 als „deutlich erhöht“, von 
70 bis 74 als „stark erhöht“ und von 75 bis 80 (und höher) als „,sehr stark erhöht“ interpretiert. Die Skala „paranoides Denken“ ist somit im Durchschnitt bei den Patientinnen nur leicht erhöht, alle anderen Skalen und globalen Kennwerte sind im Mittel deutlich oder sogar stark erhöht. Zusätzlich kann die SCL-90-R auch anhand von Derogatis` Falldefinition betrachtet werden. Somit sind GSI-T-Werte $\geq 63$ und/oder mindestens zwei Skalen mit TWerten $\geq 63$ als „Fall“ und damit als auffällige psychische Belastung zu interpretieren. Das Kollektiv erreicht im Mittel einen GSI-T-Wert von 71 und liegt damit weit über dem Cutoff-Wert der Falldefinition. Insgesamt kann davon ausgegangen werden, dass das Followup-Kollektiv im Durchschnitt auffällig psychisch belastet war.

Für die Auswertung des Beck'schen-Depressions-Inventars wurden alle Itemwerte aufsummiert und aus den Summenwerten aller 26 Patientinnen ein Mittelwert von 23,8 (SD = 10,9) ermittelt. In der S3-Leitlinie/NVL Unipolare Depression werden folgende Cut-off-Werte bei dem BDI empfohlen (DGPPN et al. 2015): Unter zehn Punkten wird davon ausgegangen, dass keine Depression vorliegt bzw. der Patient klinisch unauffällig oder remittiert ist. Von 10 bis 19 Punkten wird von einem leichten depressiven Syndrom gesprochen, bei Punkten zwischen 20 bis 29 wird die Depression als mittelgradig eingestuft. Alle Punktzahlen über 30 werden schließlich als schweres depressives Syndrom interpretiert. Nach dieser Einteilung hatten acht Patientinnen ein schweres depressives Syndrom, sieben ein mittelgradiges und neun ein leichtes; zwei Patientinnen fielen unter die 10 Punkte-Grenze und waren damit klinisch unauffällig. Durchschnittlich ist das Kollektiv einem mittelgradig depressiven Syndrom zuzuordnen. Diese Ergebnisse stimmen mit den klinischen Daten der Patientinnen überein: Bei allen Patientinnen ist die Diagnose einer rezidivierenden depressiven Störung vorbeschrieben.

Tabelle 8: Mittelwerte und Standardabweichungen zu den IES-R-Skalen des Kollektivs zum Zeitpunkt Follow-up $(N=25)$. Zum Vergleich werden Werte von nichttraumatisierten Gesunden (Maercker 2002, S. 205) und von Frauen mit sexuellen Gewalterfahrungen (Maercker 2002, S. 205 nach Wise 2000) aufgeführt.

\begin{tabular}{|l|c|c|c|c|c|c|}
\hline \multirow{2}{*}{ IES-R } & \multicolumn{3}{|l|}{ Follow-up-Kollektiv } & \multicolumn{2}{|c|}{$\begin{array}{c}\text { Vergleichswerte: } \\
\text { Nichttraumatisierte } \\
\text { Gesunde }\end{array}$} & \multicolumn{2}{|c|}{$\begin{array}{c}\text { Vergleichswerte: } \\
\text { Frauen mit sexuellen } \\
\text { Gewalterfahrungen }\end{array}$} \\
\cline { 2 - 8 } & Mittelwert & $S D$ & Mittelwert & $S D$ & Mittelwert & $S D$ \\
\hline Skala Intrusion & 23,4 & 9,5 & 6,1 & 5,3 & 28,4 & 5,8 \\
\hline Skala Vermeidung & 22,2 & 8,2 & 6,6 & 7,0 & 25,9 & 6,7 \\
\hline Skala Übererregung & 21,7 & 8,4 & 6,9 & 8,9 & 27,8 & 6,0 \\
\hline
\end{tabular}

Für die IES-R wurden die drei Subskalen Intrusion, Vermeidung und Übererregung berechnet, die Auswertung eines Gesamtscores hat sich nicht durchgesetzt. Die Maximalwerte für Intrusion und Übererregung sind 35 Punkte, für Vermeidung 40 Punkte. Für die IES-R gibt es keine Normwerte, es liegen jedoch Vergleichswerte von nichttraumatisierten Gesunden sowie von Frauen mit sexuellen Gewalterfahrungen vor (s. Tab. 8). Die Skalenwerte des Follow-up-Kollektivs sind im Durchschnitt ein Vielfaches höher als die der nichttraumatisierten 
Gesunden. Sie erreichen jedoch nicht die Vergleichswerte, die für Frauen mit sexuellen Gewalterfahrungen vorliegen. Insgesamt ist von einem pathologischen Ergebnis auszugehen. Mit der im Kapitel 3.2.1.3 aufgeführten Formel von Maercker und Schützwohl (1998) lässt sich - bei positivem Ergebnis - die Verdachtsdiagnose einer PTBS vermuten. Von den 25 Patientinnen, die die IES-R ausgefüllt hatten, war dies bei 13 Patientinnen der Fall.

Die Ergebnisse des FDS sind bei fehlender Normalverteilung in Form von Median und Quartilen in Tabelle 9 dargestellt. Rodewald et al. (2006) empfehlen in ihrer Arbeit einen Cut off-Wert von 13 für den FDS und von 15 Punkten für den DES. Im Schnitt liegen die Patientinnen weit über diesem Wert. 14 der 26 Patientinnen hatten einen FDS-Wert über 13 und einen DES-Wert über 15.

Tabelle 9: Mediane und Quartile zu den FDS- und DES-Gesamtskalenwerten sowie zu den FDSSubskalen des Kollektivs zum Zeitpunkt Follow-up $(N=26)$

\begin{tabular}{|l|c|c|c|}
\hline \multicolumn{1}{|c|}{ FDS } & Median & $\begin{array}{c}\text { Oberes } \\
\text { Quartil }\end{array}$ & $\begin{array}{c}\text { Unteres } \\
\text { Quartil }\end{array}$ \\
\hline Dissoziative Amnesie & 3,8 & 17,8 & 1,3 \\
\hline Tendenz zu imaginativen Erlebnisweisen & 18,9 & 41,1 & 10,0 \\
\hline Depersonalisation/Derealisation & 12,5 & 25,4 & 4,2 \\
\hline Skala für pseudoneurol. Konversionssymptome & 9,4 & 25,8 & 1,1 \\
\hline Skala für ICD-10-Symptome & 11,2 & 22,6 & 3,1 \\
\hline FDS-Gesamtskalenwert & 14,1 & 26,0 & 6,6 \\
\hline DES-Gesamtskalenwert & 18,2 & 29,0 & 7,0 \\
\hline
\end{tabular}

Zur besseren Einordnung sind in Tabelle 10 Vergleichsmittelwerte aus einer vorangegangenen Studie in Deutschland aufgeführt (Rodewald et al. 2006, S. 255). Mit einem FDSMedian von 14,1 und einem DES-Median von 18,2 liegt das Follow-up-Kollektiv hiernach weit über den Werten der Allgemeinbevölkerung, aber unter den Werten von Patientinnen mit spezifischen dissoziativen Störungen.

Tabelle 10: FDS- und DES-Vergleichsmittelwerte verschiedener Stichproben aus einer Studie in Deutschland (Rodewald et al. 2006, S. 255)

\begin{tabular}{|l|c|c|}
\hline \multicolumn{1}{|c|}{ Stichprobe } & $\begin{array}{c}\text { Mittlerer } \\
\text { FDS-Wert }\end{array}$ & $\begin{array}{c}\text { Mittlerer } \\
\text { DES-Wert }\end{array}$ \\
\hline Patientinnen mit dissoziativer Identitätsstörung $(N=44)$ & 40,1 & 45,4 \\
\hline Patientinnen mit DDNOS vom Typ 1 $(N=22)$ & 25,4 & 29,0 \\
\hline Patientinnen mit posttraumatischen Störungsbildern $(N=20)$ & 10,4 & 12,8 \\
\hline $\begin{array}{l}\text { Patientinnen mit traumaunspezifischen Störung wie Angst- } \\
\text { störungen und affektive Störungen }(N=34)\end{array}$ & 5,7 & 6,4 \\
\hline Allgemeinbevölkerung $(N=30)$ & 2,9 & 4,0 \\
\hline
\end{tabular}

Anhand des Manuals von Leichsenring wurden die Mittelwerte des BPI in T-Werte transformiert sowie die zugehörigen Prozentränge ermittelt (s. Tab. 11). Dazu wurde sowohl eine Stichprobe von 200 ,gesunden Probanden“ (Nicht-Patienten, überwiegend Studenten) als auch eine Stichprobe mit 67 Borderline-Patienten herangezogen. Leichsenring empfiehlt die 
Verwendung des Kriteriums Cut-20 $\geq 10$, wobei Werte von zehn oder mehr auf die Diagnose „Borderline-Störung“ hindeuten. Alle Werte unter zehn sprechen demnach für das Vorliegen einer anderen psychischen Störung oder auch keiner psychischen Störung (Leichsenring 1997). 15 der 26 Patientinnen erreichten einen Cut-20-Wert von zehn oder mehr als Hinweis auf eine „Borderline-Störung“. Im Durchschnitt erreichen die Patientinnen zusammen einen Cut-20-Wert von 10,4 und liegen somit knapp über dem Cut-off-Wert von zehn. Da bei zehn Patientinnen bereits eine Borderline-Persönlichkeitsstörung diagnostiziert worden war, überraschen diese Ergebnisse wenig.

Tabelle 11: Mittelwerte und Standardabweichungen zu den BPI-Skalen und dem BPI-Cut-20 des Kollektivs zum Zeitpunkt Follow-up $(N=26)$. Außerdem T-Werte und Prozentränge dieser Mittelwerte bezogen auf die Stichproben „Nicht-Patienten“ und „Borderline“ (Leichsenring 1997, S. 64-66)

\begin{tabular}{|l|c|c|c|c|c|c|}
\hline \multirow{2}{*}{ BPI } & \multirow{2}{*}{$\begin{array}{c}\text { Mittel- } \\
\text { wert }\end{array}$} & \multirow{2}{*}{$S D$} & \multicolumn{2}{|c|}{$\begin{array}{c}\text { Bezogen auf Stichprobe } \\
\text { „Nicht-Patienten“ }\end{array}$} & \multicolumn{2}{|c|}{$\begin{array}{c}\text { Bezogen auf Stich- } \\
\text { probe „Borderline“ }\end{array}$} \\
\cline { 4 - 8 } & & T-Werte & Prozentrang & T-Werte & Prozentrang \\
\hline Identitätsdiffusion & 5,8 & 3,5 & 63 & 89 & 47 & 36 \\
\hline Angst vor Nähe & 4,7 & 2,4 & 67 & 96 & 50 & 52 \\
\hline $\begin{array}{l}\text { Primitive Abwehr und } \\
\text { Objektbeziehungen }\end{array}$ & 3,5 & 2,4 & 67 & 96 & 43 & 24 \\
\hline $\begin{array}{l}\text { Mangelhafte } \\
\text { Realitätsprüfung }\end{array}$ & 0,8 & 1,2 & 66 & 95 & 54 & 64 \\
\hline Cut-20 & 10,4 & 4,8 & 65 & 94 & 43 & 22 \\
\hline
\end{tabular}

Im Vergleich zur Kontrollstichprobe aus gesunden Teilnehmern fallen die Skalenwerte der Patientinnen im Mittel sehr hoch aus. Sie erreichen Prozentränge von 89 bis 96 . Zieht man dagegen die Werte der Borderline-Stichprobe heran, nehmen die Patientinnen Prozentränge zwischen 24 und 64 für die Skalenmittelwerte ein und liegen somit im durchschnittlichen Bereich.

Die Ergebnisse der Borderline-Symptom-Liste sind in Tabelle 12 aufgelistet. Anhand von Prozenträngen können die Werte mit denen einer BPS-Vergleichsstichprobe in Bezug gesetzt werden, die von der Borderline Research Unit am ZI Mannheim zusammen mit weiteren Auswertungshinweisen für die BSL veröffentlicht wurde. Die Vergleichsstichprobe wurde 1997 erhoben und bestand aus 308 Frauen mit einem Durchschnittsalter von 30,3 (SD = 7,5) Jahren, die die DSM-IV-Kriterien einer Borderline-Störung erfüllten (Borderline Research Unit ZI-Mannheim 2009). Bei der Gesamtskala erreichten die Patientinnen im Durchschnitt einen Prozentrang von 27. Im Mittel hatten die Patientinnen somit eine höhere BorderlineSymptomlast als $26 \%$ der Normierungsstichprobe, aber auch eine geringere Belastung als die restlichen $72 \%$. Auffällig ist, dass das Follow-up-Kollektiv mit einem Prozentrang von 57 vor allem bei Intrusionen hohe Werte erreicht. 
Tabelle 12: Mittelwerte und Standardabweichungen zu den BSL-Subskalen und der BSL-Gesamtskala des Kollektivs zum Zeitpunkt Follow-up $(N=26)$. Außerdem die Prozentränge der Mittelwerte bezogen auf die BPS-Vergleichsstichprobe (Borderline Research Unit ZI-Mannheim 2004)

\begin{tabular}{|l|c|c|c|}
\hline \multicolumn{1}{|c|}{ BSL } & Mittelwert & SD & Prozentrang \\
\hline Selbstwahrnehmung & 23,8 & 17,5 & 38 \\
\hline Affektregulation & 22,7 & 13,3 & 28 \\
\hline Autoaggression & 16,8 & 12,3 & 31 \\
\hline Dysphorie & 31,7 & 6,7 & 45 \\
\hline Soziale Isolation & 14,1 & 9,7 & 33 \\
\hline Intrusionen & 11,7 & 9,1 & 57 \\
\hline Feindseligkeit & 6,6 & 4,7 & 38 \\
\hline BSL-Gesamtskala & 148,6 & 73,2 & 27 \\
\hline
\end{tabular}

\subsection{Prüfung von Voraussetzungen für die statistische Auswertung}

\subsubsection{Zeitspanne zwischen Baseline und Follow-up}

Die Baseline-Untersuchung fand in den Jahren 2009/2010 statt, 2013 wurde das Follow-up durchgeführt. Ziel war es, einen etwa dreijährigen Abstand zwischen Baseline- und Followup-Untersuchung einzuhalten. Im Mittel lagen 38,8 ( $S D=3,6)$ Monate zwischen den beiden Untersuchungen. Das Minimum an Abstand lag bei 34, das Maximum bei 46 Monaten. Da die Streuung nicht sehr groß ist, kann davon ausgegangen werden, dass in der Regel etwas mehr als drei Jahre zwischen den beiden Untersuchungen lagen.

\subsubsection{Vergleich der Gruppe „Follow-up“ mit der Gruppe „Nur Baseline“}

Da nur etwa die Hälfte der Baseline-Teilnehmerinnen auch an dem Follow-up teilnahm, stellt sich die Frage, ob ein systematischer Unterschied zwischen den Teilnehmerinnen an beiden Untersuchungen (Baseline und Follow-up) und den restlichen Patientinnen, die nur zu der Baseline-Untersuchung kamen, besteht (s. Abb. 6). Getestet wurde dies bei mindestens intervallskalierten Variablen mit einem unabhängigen t-Test, während für kategoriale Variablen ein Chi-Quadrat-Test Anwendung fand. Alle Ergebnisse der Testung sind tabellarisch im Anhang 7.1 aufgelistet.

Es wurden alle soziodemographischen und - soweit testbar - die klinischen Daten sowie das Stressempfinden in der Baseline-Messung und die Gesamtwerte der psychometrischen Fragebögen zum Zeitpunkt Baseline betrachtet. Zusätzlich wurden auch die fünf für die weiteren Analysen ausgewählten Parameter HR, sBP, TPRI, PEP und BRS (s. Kap. 3.2.2.3) jeweils in der ersten Phase der Baseline-Messung (Ruhe 1) in die Testung miteinbezogen. So sollte festgestellt werden, ob sich die beiden Gruppen in ihren hämodynamischen und autonomen Werten grundsätzlich unterscheiden. Alle metrischen Variablen wurden zunächst auf Varianzgleichheit getestet, die überwiegend von dem Levene-Test bestätigt wurde. 


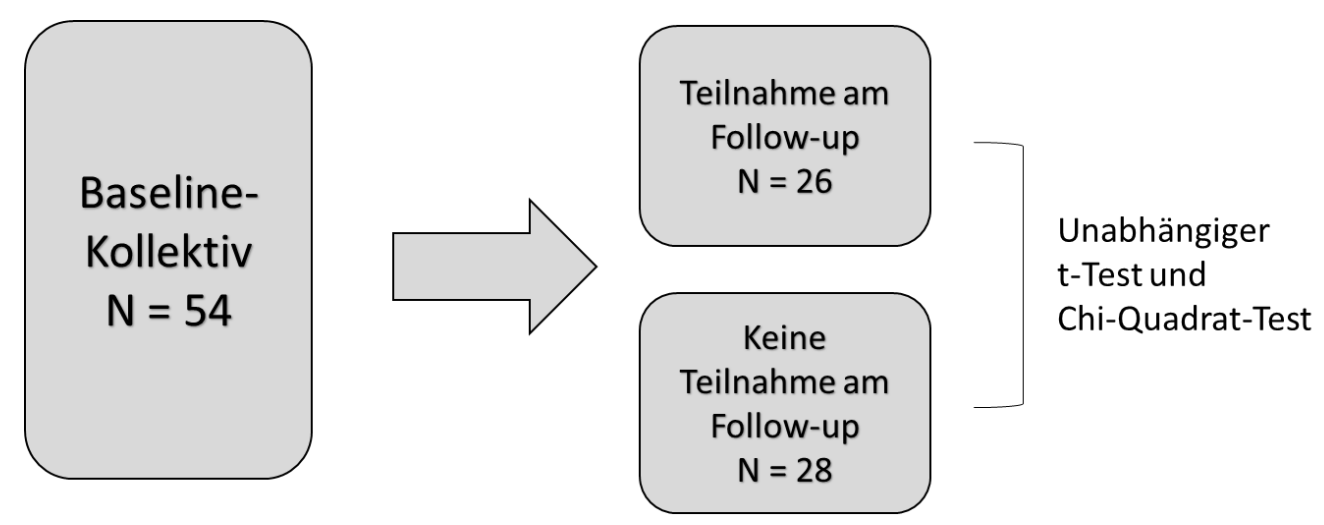

Abbildung 6: Vergleich der Gruppe „Follow-up“ mit der Gruppe „Nur Baseline“: 26 Patientinnen nahmen an beiden Untersuchungen teil, 28 Patientinnen nur an der Baseline-Untersuchung. Zwischen diesen beiden Gruppen erfolgt ein Vergleich mittels unabhängigen t-Tests und ChiQuadrat-Tests.

Ausnahme war einzig die Variable „Alkoholkonsum“, zu der daher im t-Test die Werte für eine angenommene Varianzinhomogenität herangezogen wurden. Daraufhin wurde der unabhängige t-Test für alle metrischen Variablen durchgeführt. Es fand sich bei keiner der Variablen ein signifikanter Unterschied zwischen den beiden Gruppen („Baseline und Follow-up“ und „Nur Baseline“").

Für den Chi-Quadrat-Test muss teilweise eine eingeschränkte Aussagekraft beachtet werden, denn es traf bei manchen kategorialen Variablen eine zu geringe Stichprobengröße auf eine zu große Anzahl an Kategorien. Der Chi-Quadrat-Test wird aber in der Regel nur als aussagekräftig angenommen, wenn eine erwartete Anzahl von über fünf pro Kategorie vorliegt. Dieses Problem ist nur mit einer größeren Stichprobe lösbar, welche jedoch aufgrund des Studiendesigns und der begrenzten Rekrutierungsmöglichkeiten nicht erreichbar war. Der Chi-Quadrat-Test wurde trotz dieser Einschränkungen bei allen kategorialen Variablen durchgeführt; mehr als eine grobe Betrachtung der Gruppenunterschiede wird jedoch nicht aus ihm abgeleitet. Soweit es sinnvoll möglich war, wurden bei Variablen Kategorien zusammengefasst, sodass bei diesen Variablen die erwartete Anzahl pro Kategorie über fünf lag oder zumindest erhöht wurde.

Bei keiner der im Chi-Quadrat-Test überprüften Variablen waren die Unterschiede zwischen den beiden Gruppen signifikant bzw. lagen nahe des Signifikanzniveaus. Einzige Ausnahme war die Variable „PTBS-Typ“ (Typ 1-Trauma, Typ 2-Trauma oder Typ 1- und Typ 2-Trau$\mathrm{ma}$ ), die mit $p=0,051$ nur knapp nicht signifikant war. Bei einem Ergebnis so nahe am Signifikanzkriterium lohnt sich ein genauerer Blick mithilfe des Exakten Fisher-Tests. Hiermit wird der Gruppenvergleich für diese Variable signifikant $(p<0,05)$. Die Gruppen unterscheiden sich in erster Linie darin, dass unter den Patientinnen, die nicht am Follow-up teilnahmen, keine Patientin mit einem Typ 1-Trauma ist, während es bei den Patientinnen mit 
Follow-up-Teilnahme drei sind. Dafür zählen zu der Gruppe „Nur Baseline“ neun Patientinnen, die sowohl ein Typ 1- als auch ein Typ 2-Trauma erlebten, während bei der Gruppe „Baseline und Follow-up“ nur drei Patientinnen beide Arten an Trauma angegeben hatten.

Insgesamt konnten keine signifikanten systematischen Unterschiede zwischen den Teilnehmern am Follow-up und den restlichen Patientinnen festgestellt werden. Einzige Ausnahme war die Variable „PTBS-Typ“, bei der im Exakten Fisher-Test ein signifikanter Unterschied resultierte. Da alle anderen Variablen jedoch unauffällig waren, wird im Folgenden davon ausgegangen, dass keine wesentlichen Unterschiede bestehen, die bei den Analysen und der Interpretation der Ergebnisse beachtet werden müssen. Von der Repräsentativität der Follow-up-Teilstichprobe für die Baseline-Ausgangsstichprobe ist auszugehen.

\subsubsection{Prüfung auf Normalverteilung}

Es wurden die Differenzen aus den Variablen der Baseline-Untersuchung und den jeweiligen zugehörigen Variablen der Follow-up-Untersuchung gebildet und auf Normalverteilung getestet. Hierbei wurden nur Variablen einbezogen, die in der weiteren Auswertung eine Rolle spielen. In der überwiegenden Mehrzahl wurde im Shapiro-Wilk-Test eine Normalverteilung bestätigt. Signifikant und damit nicht normalverteilt waren die Differenzen folgender Variablen:

- die Skala „Vermeidung“ der IES-R

- die Skalen „Primitive Abwehr und Objektbezogenheit“ und „Mangelhafte Realitätsprüfung" des BPI

- alle Skalen und Kennwerte des FDS

- der systolische Blutdruck und der TPRI in der Phase 4

- die Baroreflexsensitivität in der Phase 1

\subsubsection{Bezugsgrößen für die Stressreagibilität}

Für die Bildung der $\Delta \mathrm{R}$-Scores zur Veränderung der Reagibilität zwischen den beiden Messzeitpunkten ist es entscheidend, die Messphasen zu identifizieren, die die maximale Stressreagibilität der Patientinnen abbilden. Zum einen dienen dazu natürlich die beiden Stressphasen, sowohl das Rechnen als auch das Babyschreien sollen betrachtet werden. Zum anderen wird eine Phase mit einer möglichst geringen Stressaktivierung als Bezugsgröße benötigt - eine Messphase, die am ehesten Ruhebedingungen entspricht. Nur so kann die maximale Änderung in Bezug auf die Stressaktivierung und somit die Stressreagibilität bestimmt werden. Hierzu kommen zwei Phasen als mögliche Bezugsgröße in Frage: Ruhe 1 und Entspannungsmusik. Die Phasen Ruhe 2 und Ruhe 3 erscheinen dagegen als ungeeignet, da sie jeweils direkt hinter einer Stressphase liegen und somit noch deutlich durch die zuvor erfolgte Stressreaktion beeinflusst sind. Ruhe 1 dagegen liegt vor dem Stresstest und wird für die Bestimmung der Reactivity, der Aktivierung durch die Stressoren (s. Kap. 4.3.5), verwendet. Die Phase Entspannungsmusik befindet sich am Ende der Untersuchung und somit mit 
deutlichem zeitlichem Abstand zu den Stressphasen. Sie ist Grundlage für die Berechnung der Recovery, der Erholung nach der Stressreaktion (s. Kap. 4.3.6). Die Entscheidung, welche Phase im Sinne von Ruhebedingungen als Bezugsgröße für die Berechnung der Scores zur Reagibilität und damit zur Bestimmung der Regulationsbreite dienen soll, soll rein statistisch getroffen werden. Durch einen direkten Vergleich von Ruhe 1 und Entspannungsmusik soll die Phase ausgewählt werden, die im Mittel mit der geringsten kardiovaskuläre Aktivierung einhergeht.

Eine 2 x 2 ANOVA für Messwiederholungen mit den Faktoren Zeit (Baseline vs. Followup) und Ruhephasen (Ruhe 1 vs. Entspannungsmusik) wurde für die fünf Parameter (HR, sBP, TPRI, PEP und BRS) durchgeführt (s. Tab. 13). Es sei angemerkt, dass sich die Mittelwerte und Standardabweichungen der Phase Ruhe 1 von an anderer Stelle berichteten Zahlen (Kap. 4.3.3, 4.3.4 und 4.3.5) unterscheiden. Grund hierfür ist der Abbruch einer Patientin in der Baseline-Messung, die daher für alle Berechnungen mit der Phase Musik (Baseline) ausgeschlossen wurde (s. Kap. 4.1.1). Zudem sind Unterschiede zu den Kapiteln 4.3.5. und 4.3.6 aufgrund des Ausschlusses von Patientin E1 (s. Kap. 4.2.5) zu beachten.

Tabelle 13: „Ruhe“-ANOVA. Phasenmittelwerte und Standardabweichungen der Phasen Ruhe 1 und Entspannungsmusik (jeweils für Baseline und Follow-up) und eine 2 × 2 ANOVA für Messwiederholung für die Parameter HR in [bpm], sBP in $\left[\mathrm{mmHg}\right.$, TPRI in $\left[\mathrm{dyn}^{*} \mathrm{~s}^{*} \mathrm{~m}^{2} / \mathrm{cm}^{5}\right]$, PEP in [ms] und BRS in $[\mathrm{ms} / \mathrm{mmHg}]$.

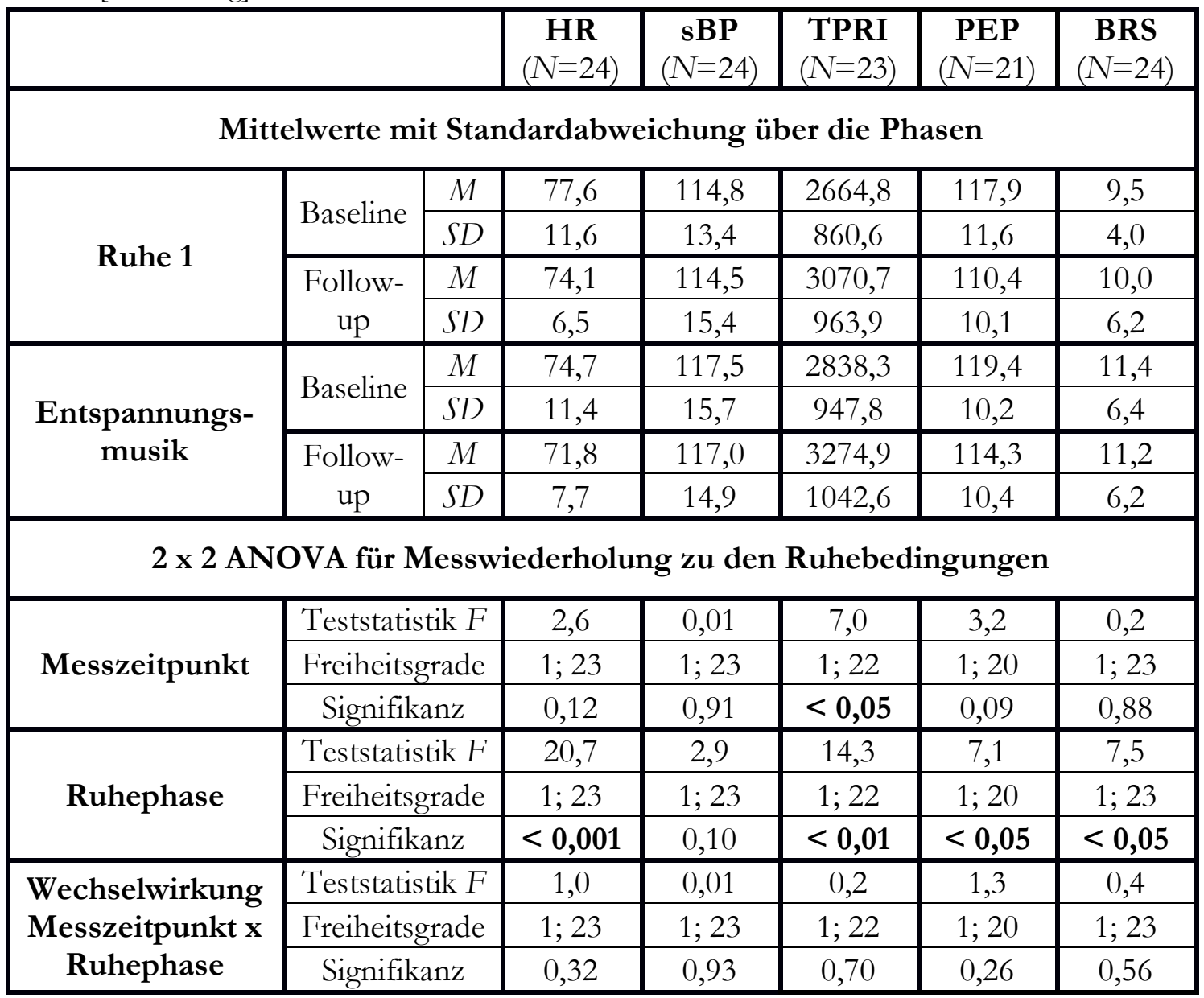


Die Phasen Ruhe 1 und Entspannungsmusik unterscheiden sich signifikant bis höchstsignifikant bei fast allen Parametern. Einzig bei dem systolischen Blutdruck besteht kein signifikanter Effekt. Die Herzrate war in der Phase Musik geringer, während die BRS und der PEP in dieser Phase höhere Werte als in Ruhe 1 zeigten. Dies alles spricht für eine stärkere Entspannung bei der Entspannungsmusik im Vergleich zur Phase Ruhe 1. Der TPRI dagegen lag in der Phase Musik höher, welches einer vermehrten alpha-adrenergen Sympathikusreaktion bei der Entspannungsmusik entsprechen könnte. Passend hierzu lag auch der sBP, wenn auch insignifikant, in der Phase Musik über der Phase Ruhe 1. Zudem ist der TPRI der einzige Parameter, bei dem sich ein signifikanter Zeiteffekt findet.

Insgesamt überwiegen die Anzeichen, dass die Phase Entspannungsmusik eher Ruhebedingungen und damit einer geringeren kardiovaskulären Aktivierung entspricht als die Phase Ruhe 1. Sie wird somit für die Berechnung der Scores zur Reagibilität verwendet. Da somit insbesondere die Recovery betrachtet wird, werden die Scores im Folgenden als Recoveryscores und $\Delta \mathrm{R}$-Scores zur Recovery bezeichnet. Die Reagibilität wird somit im Folgenden insbesondere über die Recovery bestimmt.

\subsubsection{Graphische Betrachtung der $\Delta \mathrm{R}$-Scores zur Detektion von Extremwerten}

Für das Konzept und die Berechnung der Scores zur Änderung der Recovery bzw. Reagibilität $\Delta \mathrm{R}_{\text {Rechnen }}$ und $\Delta \mathrm{R}_{\text {Babyschreien }}$ sei an dieser Stelle auf das Kapitel 3.5.1 verwiesen. Bevor in dem Kapitel 4.5 eine Auswertung der $\Delta \mathrm{R}$-Scores erfolgen kann, soll zur Detektion von Extremwerten eine graphische Betrachtung der $\Delta \mathrm{R}$-Scores für die verschiedenen Parameter mithilfe von Boxplots vorgenommen werden. Das Statistikprogramm SPSS stellt Werte als Ausreißer im Boxplot dar, wenn ihr Abstand von dem 25. Perzentil nach unten bzw. von dem 75. Perzentil nach oben zwischen dem 1,5-fachen und 3-fachen Interquartilsabstand liegt. Extremwerte dagegen sind über dem 3-fachen Interquartilsabstand von dem 25. oder dem 75. Perzentil entfernt (Brosius 2011).

Es finden sich mehrere Extrem- und Ausreißerwerte, wobei nur der Ausschluss von Extremwerten diskutiert werden soll. In Abbildung 7 und 8 sind die Extremwerte einer Patientin (im Folgenden als Patientin E1 bezeichnet) mit E1 gekennzeichnet. Die Patientin wich somit bei den Scores $\Delta R_{S B P}$ Babyschreien und $\Delta R_{T P R I}$ Babyschreien weit von dem restlichen Kollektiv ab. Ein solcher Extremwert kann gerade bei einem kleinen Kollektiv die Ergebnisse beträchtlich beeinflussen, sodass eine genauere Betrachtung der Patientin E1 erforderlich ist. 


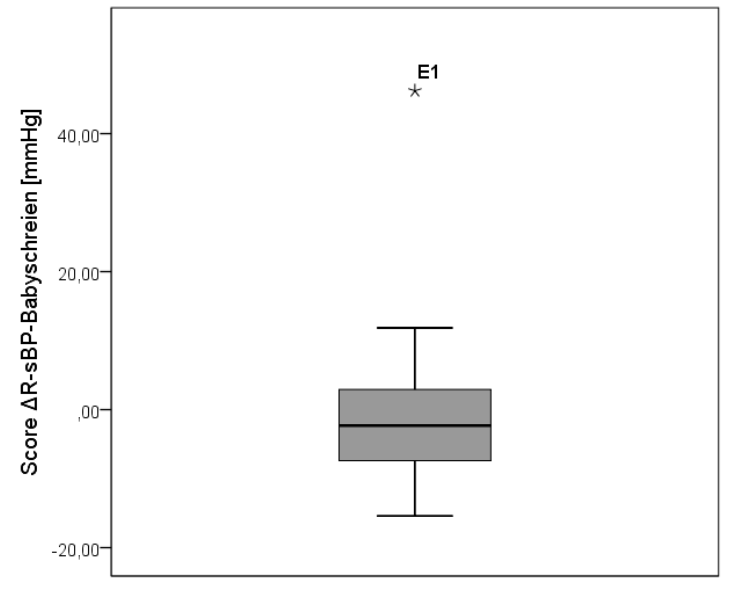

Abbildung 7: Score $\Delta R_{S B P}$ Babyschreien in $[\mathrm{mmHg}]$ als Boxplot dargestellt. E1 bezeichnet einen Extremwert der Patientin E1

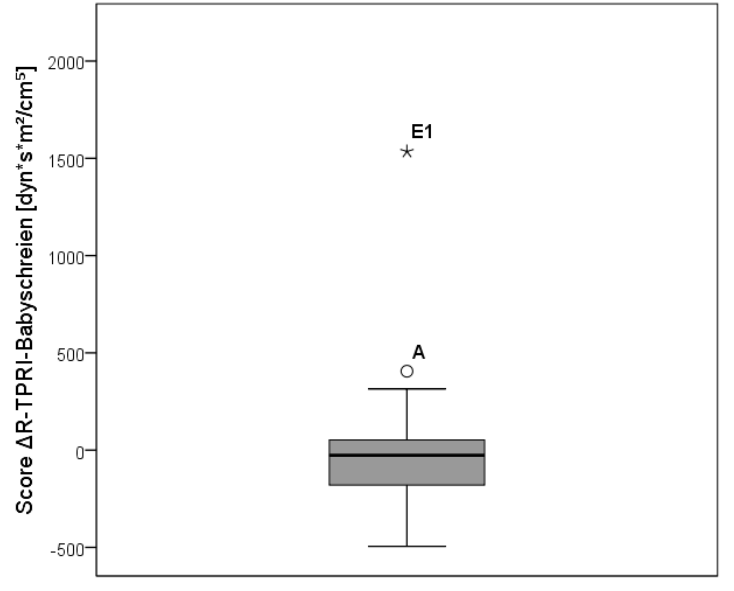

Abbildung 8: Score $\Delta R_{T P R I \text { Babyschreien }}$ in [dyn*s* $\mathrm{m}^{2} / \mathrm{cm}^{5}$ ] als Boxplot dargestellt. Mit E1 ist ein Extremwert der Patientin E1 benannt, A bezeichnet einen Ausreißer.

Die Patientin E1 hatte in der Baseline-Untersuchung in der Phase Babyschreien einen sehr starken Blutdruck- und TPRI-Anstieg gezeigt, ganz im Gegensatz zur Follow-up-Untersuchung, wo sie über die ganze Messung hinweg nur relativ niedrige systolische Blutdruckwerte erreichte. Bei der Patientin war bereits bei der Baseline-Untersuchung ein arterieller Hypertonus diagnostiziert und sie nahm zu diesem Zeitpunkt einen Calciumantagonisten, einen ACE-Hemmer, ein Neuroleptikum sowie ein Antidepressivum ein. Im Follow-up dagegen umfassten ihre Medikamente einen Betablocker, ein Neuroleptikum sowie zwei Antidepressiva (ein trizyklisches Antidepressivum und ein SSRI). Entscheidend ist hier das Hinzukommen des Betablockers, der die Stressreagibilität durchaus beeinflusst haben könnte. Dies sowie der sehr große Abstand zum restlichen Kollektiv und die Tatsache, dass sich gleich bei zwei Parametern Extremwerte finden, führen zu der Entscheidung die Patientin E1 aus allen deskriptiven Betrachtungen der $\Delta \mathrm{R}$-Scores zur Änderung der Reagibilität sowie aus allen Korrelationsberechnungen mit diesen auszuschließen. Im Anhang sind die Ergebnisse mit Einschluss von Patientin E1 für einen Vergleich aufgelistet. Sie zeigen, dass der Ausschluss zu einer konservativeren Betrachtung führt. Da mit den ANOVAs in den Kapiteln 4.3.5 und 4.3.6 die Aktivierung durch die Stressoren bzw. die Recovery-Reaktion zu den beiden Messzeitpunkten und damit ebenfalls die Stressreagibilität betrachtet wird, wird die Patientin E1 folglich auch aus diesen Berechnungen ausgeschlossen. Auch hier kann der Ausschluss durch einen Vergleich mit den Tabellen im Anhang evaluiert werden, hier finden sich beide ANOVAs unter Einschluss der Patientin E1. Für einen generellen Ausschluss der Patientin E1 fehlte jedoch die Grundlage, da sie in den Ruhephasen keine Extremwerte verzeichnete. Es sei jedoch auf eine Sensitivitätsanalyse im Anhang verwiesen, die sich auf einen möglichen Einfluss auf die Ergebnisse durch Änderungen in der Betablockermedikation konzentriert. 
Ein weiterer Extremwert (s. Abb. 9, Punkt E2) ist der Score $\Delta R_{B R S}$ Rechnen der Patientin „E2“. Im Gegensatz zu der Patientin E1 sind bei ihr keine kardiovaskulären Vorerkrankungen oder Medikamenteneinnahmen bekannt. Lediglich ein Antidepressivum wurde beim Follow-up von ihr eingenommen. Auch finden sich bei keinem weiteren $\Delta \mathrm{R}$-Score bei der Patientin E2 Abweichungen. Warum sie so weit von dem Kollektiv abweicht, ist somit nicht ersichtlich, es wurde sich daher gegen einen Ausschluss entschieden. Um jedoch den Einfluss, den die Patientin E2 auf die Ergebnisse hat, einschätzen zu können, wurden alle Korrelationen zusätzlich auch ohne sie gerechnet (s. Anhang, Kap. 8.5).

Zuletzt finden sich auch bei dem $\Delta R_{P E P}$ Babyschreien mehrere Extremwerte (Abb. 10, E3 E5) sowie Ausreißer. Dies scheint jedoch eher dem geringen Interquartilsabstand geschuldet, als dass hier wirkliche Abweichler vorlägen. Ein Blick auf $\triangle R_{P E P}$ Rechnen bestätigt, dass Werte, die bei $\Delta R_{P E P \text { Babyschreien }}$ als Extremwerte gelten, auch dort erreicht werden und dabei im dreifachen Interquartilsabstand liegen. Von einem Ausschluss der Patientinnen „E3 - E5“ wird daher abgesehen.

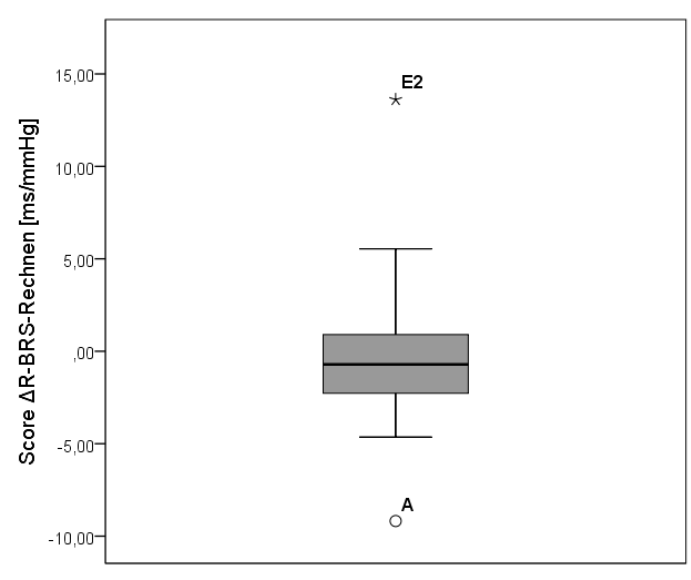

Abbildung 9: Score $\Delta R_{B R S \text { Rechnen }}$ in $[\mathrm{ms} / \mathrm{mmHg}]$ als Boxplot dargestellt. Mit E2 ist ein Extremwert der Patientin E2 gekennzeichnet, A ist ein Ausreißer.

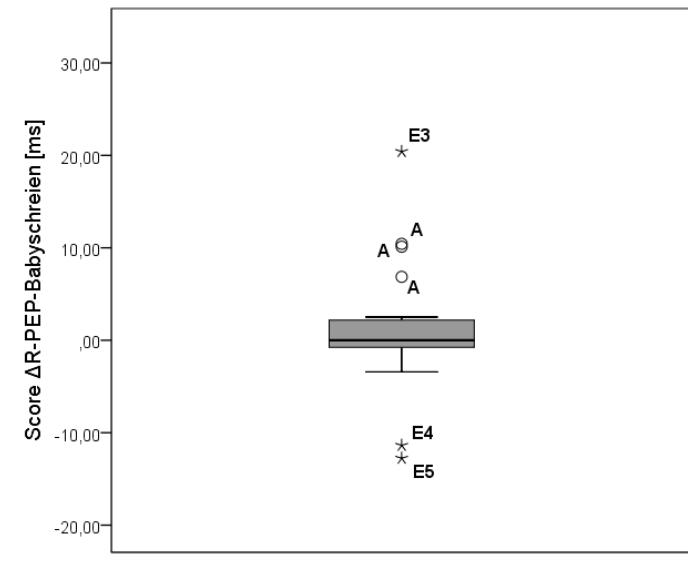

Abbildung 10: Score $\Delta R_{P E P \text { Babyschreien }}$ in [ms] als Boxplot dargestellt. Mit E3 bis E5 sind Extremwerte verschiedener Patientinnen gekennzeichnet. A steht jeweils für einen Ausreißerwert. 


\subsection{Baseline und Follow-up im Vergleich}

\subsubsection{Veränderungen im Stressempfinden (Hypothese 1)}

Die Patientinnen gaben jeweils in der Phase Ruhe 1 sowie kurz nach den Phasen Rechnen, Babyschreien und Entspannungsmusik ihr Stressempfinden auf einer Skala von Null bis Zehn $(0=$,gar kein Stress“, $10=$,größter vorstellbarer Stress“) an. In Hypothese 1 wird erwartet, dass sich bei dem Follow-up im Mittel niedrigere Werte finden als in der BaselineUntersuchung und die Patientinnen somit ein insgesamt geringeres Stressempfinden beschreiben. Die Abbildung 11 bietet einen ersten graphischen Überblick über das Stressempfinden in den verschiedenen Messphasen zu den beiden Messzeitpunkten. Es wird deutlich, dass die Patientinnen über die ganze Messung hinweg während des Follow-ups im Median weniger Stress empfanden als während der Baseline-Untersuchung. Die beiden Stressoren Rechnen und Babyschreien lösten einen ähnlich hoch wahrgenommenen Stress bei den Patientinnen aus. Das Stressempfinden streute jedoch beim Babyschreien, besonders im Follow-up, deutlich mehr. Bei der Phase Entspannungsmusik war das Stressniveau der Patientinnen nur geringfügig höher als in Ruhe 1.

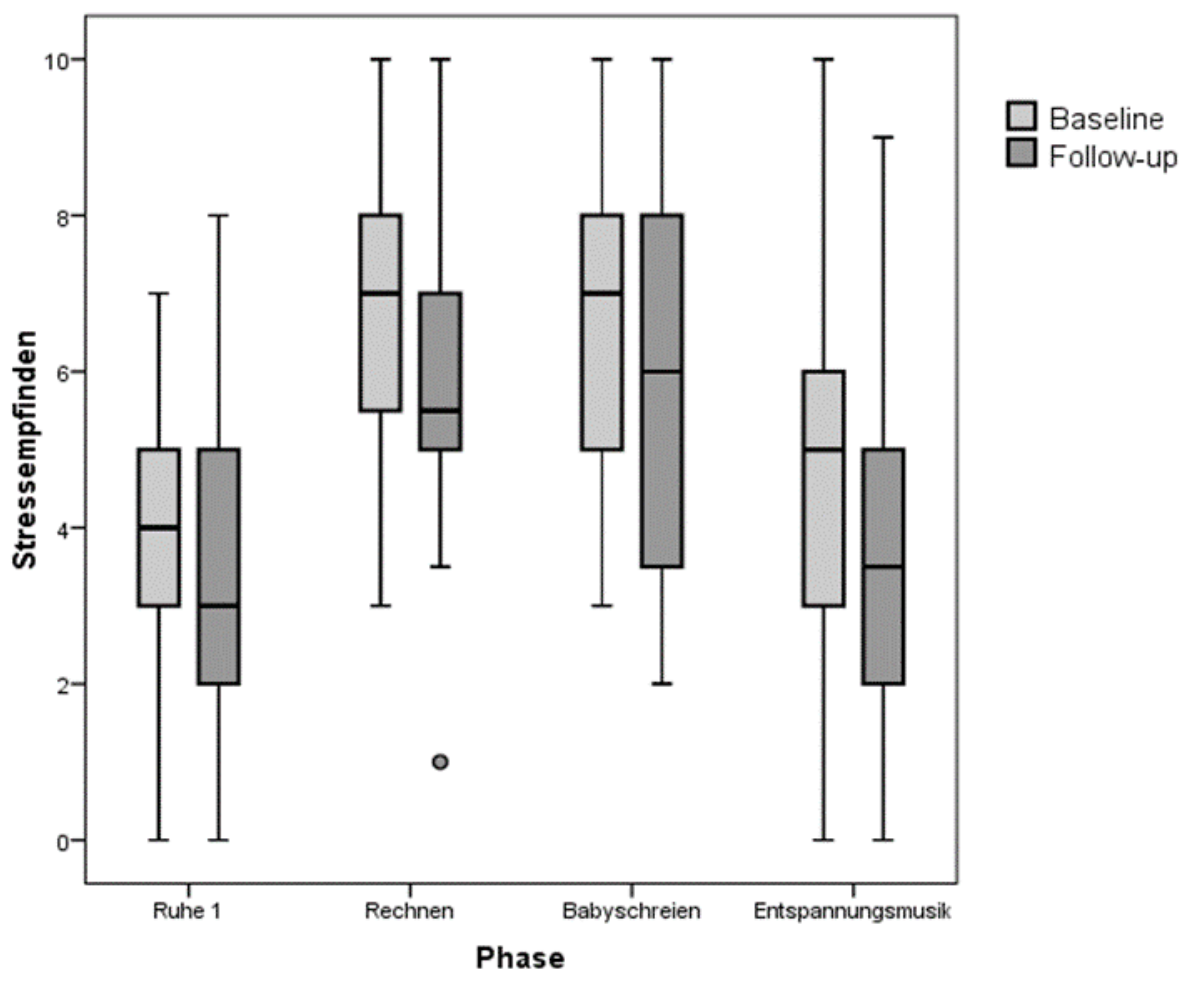

Abbildung 11: Stressempfinden des Follow-up-Kollektivs in den verschiedenen Messphasen; verglichen wird die Baseline- mit der Follow-up-Messung. Null steht für „keinen Stress“, Zehn für den ,größten vorstellbaren Stress“ 
Mit einem abhängigen t-Test wurde nun auf Unterschiede in der Stressempfindung zwischen den beiden Messzeitpunkten getestet. Die im Folgenden bezüglich des abhängigen t-Test angegebenen Signifikanzen sind aufgrund der gerichteten Hypothese einseitige Signifikanzen. Das Stressempfinden lag bei der Messung Baseline in Ruhe 1 im Mittel bei 3,9 (SD $=1,7)$, während sich beim Follow-up ein niedrigerer Mittelwert von 3,6 $(S D=2,1)$ findet. Der Unterschied zwischen den beiden Messzeitpunkten ist jedoch im abhängigen t-Test nicht signifikant $(t(24)=0,5 ; p=0,31)$. Dagegen gaben die Patientinnen während der Phase Rechnen im Follow-up im Durchschnitt ein signifikant niedrigeres Stressempfinden $(M=$ 5,9; $S D=1,8)$ als bei der Baseline-Untersuchung $(M=6,8 ; S D=1,6)$ an, $t(24)=2,0 ; p<$ 0,05. Auch bei den Phasen Babyschreien und Entspannungsmusik zeigten sich signifikante Unterschiede im Stressempfinden zwischen den beiden Messzeitpunkten. Während bei der Baseline-Messung die Patientinnen im Schnitt das Babyschreien mit einem Stress von 6,7 $(S D=2,1)$ beschrieben, verursachte dieses im Follow-up nur noch ein Stresslevel von 5,8 $(S D=2,4), t(24)=1,8 ; p<0,05$. Die Entspannungsmusik wurde im Follow-up mit mittleren 3,8 $(S D=2,5)$ als weniger stressig von den Patientinnen wahrgenommen als zum Messzeitpunkt Baseline $(M=4,7 ; S D=2,5), t(24)=2,0 ; p<0,05$.

Zur weiteren Analyse wurde eine 2 x 4 ANOVA für Messwiederholungen durchgeführt. Hierbei wurde das Stressempfinden der Patientinnen hinsichtlich der Faktoren „Messzeitpunkt“" (Baseline vs. Follow-up) und „Phase“ (Ruhe 1, Rechnen, Babyschreien und Musik) betrachtet. Die Ergebnisse zeigen, dass das Stressempfinden signifikant vom Messzeitpunkt beeinflusst wird $(F(1,24)=4,5 ; p<0,05)$. Nach Mauchlys Test war für die Testung des Faktors „Phase“ das Kriterium der Sphärizität verletzt $\left(\mathrm{X}^{2}(2)=12,0 ; p<0,05\right)$. Daher wurden die Freiheitsgrade mittels der Greenhouse-Geisser-Korrektur angepasst $(\mathcal{E}=0,8)$. Hiernach unterschied sich das Stressempfinden je nach Messphase höchst signifikant (F (2,34; $56,12)=24,2 ; p<0,001)$. Bei genauerer Betrachtung mittels eines Bonferroni adjustierten Post-hoc-Tests zeigte sich kein signifikanter Unterschied zwischen Ruhe 1 und Entspannungsmusik, dafür jedoch ein höchst signifikanter zwischen Ruhe 1 und Rechnen sowie zwischen Ruhe 1 und Babyschreien. Rechnen und Babyschreien unterschieden sich nicht signifikant. Diese Ergebnisse bestätigen den bereits gewonnenen graphischen Eindruck (Abb. 11). Hypothese 1 kann somit angenommen werden. Der Einfluss des Messzeitpunkts (Baseline oder Follow-up) interagierte nicht signifikant mit den unterschiedlichen Einflüssen der Messphasen auf das Stressempfinden $(F(3 ; 72)=0,9 ; p=0,44)$. 


\subsubsection{Veränderungen im psychometrischen Befund (Hypothese 2)}

Die Patientinnen füllten sowohl bei der Baseline- als auch bei der Follow-up-Untersuchung die psychometrischen Fragebögen aus, sodass ein Vergleich der psychischen Verfassung zu den beiden Zeitpunkten möglich ist. Hypothese 2 besagt, dass der psychometrische Befund des Follow-ups weniger pathologisch ausfällt als der der Baseline-Untersuchung. Der Vergleich erfolgt jeweils für die einzelnen Skalen und Kennwerte der Fragebögen mittels eines abhängigen t-Tests bzw. bei nicht normalverteilten Daten mittels eines WilcoxonVorzeichen-Rang-Tests. Tabelle 14 listet alle Ergebnisse des abhängigen t-Tests bzw. des Wilcoxon-Vorzeichen-Rang-Tests auf.

Tabelle 14: Vergleich von Baseline und Follow-up bzgl. der Skalenwerte und Gesamtskalenwerte aus den psychometrischen Fragebögen des Kollektivs mithilfe des abhängigen t-Tests bzw. bei fehlender Normalverteilung (jeweils mit* gekennzeichnet) mithilfe des Wilcoxon-Vorzeichen-Rang-Tests. Mit n.s. („,nicht signifikant") sind Ergebnisse gekennzeichnet, die Veränderungen entgegen der gerichteten Hypothese anzeigen (negatives $t \mathrm{bzw}$. auf negativen Rängen basierend) und damit bei der einseitigen Signifikanzangabe wegfallen. Explorativ sei jedoch angemerkt, dass keine dieser sich gegensätzlich verhaltenden Skalen einen bei zweiseitiger Testung signifikanten negativen t-Wert bzw. Z-Wert in der Testung erreichte.

\begin{tabular}{|c|c|c|c|c|c|c|c|c|}
\hline \multirow{2}{*}{\multicolumn{2}{|c|}{$\begin{array}{c}\text { Abhängiger t-Test oder } \\
\text { *Wilcoxon-Vorzeichen- } \\
\text { Rang-Test }\end{array}$}} & \multicolumn{2}{|c|}{ Baseline } & \multicolumn{2}{|c|}{ Follow-up } & \multirow{3}{*}{$\begin{array}{c}\begin{array}{c}t \\
\text { oder } \\
* Z\end{array} \\
0,3\end{array}$} & \multirow{3}{*}{$\begin{array}{c}\begin{array}{c}d f \\
\text { oder } \\
* N\end{array} \\
23\end{array}$} & \multirow{3}{*}{$\begin{array}{l}p \text { (ein- } \\
\text { seitig) } \\
0,38\end{array}$} \\
\hline & & \multirow{2}{*}{$\begin{array}{l}\text { Moder } \\
{ }^{*} M e- \\
\text { dian } \\
18,6 \\
\end{array}$} & \multirow{2}{*}{$\begin{array}{c}S D \\
\text { oder } \\
* I Q R \\
10,4\end{array}$} & \multirow{2}{*}{$\begin{array}{l}\text { Moder } \\
{ }^{*} M e^{-} \\
\text {dian } \\
18,0\end{array}$} & \multirow{2}{*}{$\begin{array}{l}S D \\
\text { oder } \\
* I Q R \\
12,0\end{array}$} & & & \\
\hline \multirow{10}{*}{$\begin{array}{l}\text { SCL } \\
-90 \\
-R\end{array}$} & Somatisierung & & & & & & & \\
\hline & Zwanghaftigkeit & 18,7 & 8,4 & 17,1 & 8,6 & 0,9 & 23 & 0,18 \\
\hline & $\begin{array}{c}\text { Unsicherheit im } \\
\text { Sozialkontakt }\end{array}$ & 17,3 & 9,4 & 14,1 & 9,6 & 1,8 & 23 & $<0,05$ \\
\hline & Depressivität & 29,3 & 14,9 & 24,8 & 12,0 & 1,6 & 23 & 0,06 \\
\hline & Ängstlichkeit & 19,1 & 9,6 & 15,3 & 10,3 & 1,9 & 23 & $<0,05$ \\
\hline & $\begin{array}{l}\text { Aggressivität/ } \\
\text { Feindseligkeit } \\
\end{array}$ & 5,2 & 4,1 & 6,4 & 5,3 & $-1,2$ & 23 & n.s. \\
\hline & Phobische Angst & 10,8 & 7,2 & 9,4 & 7,95 & 1,0 & 23 & 0,17 \\
\hline & Paranoides Denken & 7,4 & 4,8 & 7,9 & 6,0 & $-0,5$ & 23 & n.s. \\
\hline & Psychotizismus & 11,2 & 7,1 & 10,7 & 9,0 & 0,4 & 23 & 0,36 \\
\hline & GSI & 1,7 & 0,7 & 1,5 & 0,8 & 1,1 & 23 & 0,15 \\
\hline BDI & Summenwert & 29,0 & 9,1 & 24,1 & 11,0 & 2,4 & 23 & $<0,05$ \\
\hline \multirow{3}{*}{$\begin{array}{l}\text { IES- } \\
\text { R }\end{array}$} & Intrusion & 24,9 & 7,4 & 24,0 & 9,3 & 0,7 & 21 & 0,24 \\
\hline & Vermeidung* & 26,0 & 10,8 & 21,0 & 10,8 & $-1,9$ & 22 & $<0,05$ \\
\hline & Übererregung & 23,6 & 6,8 & 22,5 & 7,3 & 0,6 & 21 & 0,27 \\
\hline \multirow{5}{*}{ BPI } & Identitätsdiffusion & 5,8 & 3,5 & 5,9 & 3,6 & $-0,2$ & 23 & n.s. \\
\hline & Angst vor Nähe & 4,4 & 1,8 & 4,6 & 2,5 & $-0,5$ & 23 & n.s. \\
\hline & $\begin{array}{l}\text { Primitive Abwehr und } \\
\text { Objektbezogenheit* }\end{array}$ & 4,5 & 3,8 & 3,0 & 3,8 & $-1,7$ & 24 & $<0,05$ \\
\hline & $\begin{array}{c}\text { Mangelhafte } \\
\text { Realitätsprüfung* }\end{array}$ & 0,0 & 1,0 & 0,0 & 1,8 & $-0,1$ & 24 & n.s. \\
\hline & Cut-20 & 11,3 & 4,4 & 10,4 & 4,9 & 1,0 & 23 & 0,18 \\
\hline
\end{tabular}


Fortsetzung Tabelle 14

\begin{tabular}{|c|c|c|c|c|c|c|c|c|}
\hline \multirow{2}{*}{\multicolumn{2}{|c|}{$\begin{array}{l}\text { Abhängiger t-Test oder } \\
\text { *Wilcoxon-Vorzeichen- } \\
\text { Rang-Test }\end{array}$}} & \multicolumn{2}{|c|}{ Baseline } & \multicolumn{2}{|c|}{ Follow-up } & \multirow[b]{2}{*}{$\begin{array}{c}t \\
\text { oder } \\
* Z\end{array}$} & \multirow[b]{2}{*}{$\begin{array}{c}d f \\
o d e r \\
* N\end{array}$} & \multirow[b]{2}{*}{$\begin{array}{l}p(\text { ein } \\
\text { seitig) }\end{array}$} \\
\hline & & $\begin{array}{c}M \text { oder } \\
{ }^{*} M e- \\
\text { dian }\end{array}$ & $\begin{array}{c}S D \\
\text { oder } \\
* \text { IQR }\end{array}$ & $\begin{array}{c}\text { Moder } \\
{ }^{*} M e- \\
\text { dian }\end{array}$ & $\begin{array}{c}S D \\
\text { oder } \\
* \text { IQR }\end{array}$ & & & \\
\hline \multirow{8}{*}{ BSL } & Selbstwahrnehmung & 23,5 & 14,6 & 24,5 & 17,8 & $-0,4$ & 23 & n.s. \\
\hline & Affektregulation & 24,5 & 11,8 & 22,5 & 13,3 & 1,0 & 23 & 0,16 \\
\hline & Autoaggression & 16,8 & 12,2 & 17,2 & 12,7 & $-0,2$ & 23 & n.s. \\
\hline & Dysphorie & 32,1 & 5,4 & 31,7 & 6,9 & 0,3 & 23 & 0,38 \\
\hline & Soziale Isolation & 13,9 & 9,9 & 14,2 & 9,7 & $-0,1$ & 23 & n.s. \\
\hline & Intrusionen & 10,6 & 7,5 & 12,0 & 9,3 & $-1,0$ & 23 & n.s. \\
\hline & Feindseligkeit & 4,9 & 4,1 & 6,8 & 4,8 & $-1,6$ & 23 & n.s. \\
\hline & Gesamtskala & 148,1 & 62,3 & 150,5 & 74,5 & $-0,2$ & 23 & n.s. \\
\hline \multirow{7}{*}{ FDS } & FDS-Gesamtwert* & 17,8 & 19,1 & 14,1 & 21,5 & $-0,3$ & 24 & 0,39 \\
\hline & DES-Gesamtwert* & 21,2 & 20,6 & 18,2 & 28,1 & $-0,3$ & 24 & 0,40 \\
\hline & Dissoziative Amnesie* & 5,6 & 19,4 & 5,0 & 17,2 & $-0,3$ & 24 & n.s. \\
\hline & $\begin{array}{c}\text { Tendenz zu } \\
\text { imaginativen } \\
\text { Erlebniswelten* } \\
\end{array}$ & 27,8 & 30,6 & 17,2 & 33,0 & $-0,8$ & 24 & 0,22 \\
\hline & $\begin{array}{c}\text { Depersonalisation/ } \\
\text { Derealisation* }\end{array}$ & 20,0 & 29,2 & 14,2 & 21,3 & $-0,4$ & 24 & 0,36 \\
\hline & $\begin{array}{c}\text { Skala für pseudo- } \\
\text { neurol. Konversions- } \\
\text { symptome* }\end{array}$ & 11,1 & 22,8 & 9,4 & 21,9 & $-0,1$ & 24 & 0,47 \\
\hline & $\begin{array}{l}\text { Skala für ICD-10- } \\
\text { Symptome* }\end{array}$ & 12,6 & 20,8 & 11,2 & 19,2 & $-0,2$ & 24 & 0,43 \\
\hline
\end{tabular}

Der durchschnittliche GSI der SCL-90-R war im Vergleich zum Zeitpunkt Baseline im Follow-up gesunken und damit weniger pathologisch - diese Veränderung ist jedoch nicht signifikant. Sieben der neun Subskalen bestätigen diesen Trend und verzeichneten ebenfalls niedrigere Werte im Follow-up. Signifikant verbesserten sich hierbei einzig die beiden Subskalen Ängstlichkeit und Unsicherheit im Sozialkontakt. Allerdings erreichten auch zwei Subskalen, Aggressivität/Feindseligkeit sowie paranoides Denken, im Follow-up deskriptiv im Mittel höhere und damit pathologischere Werte als in der Baseline-Untersuchung. Insgesamt erscheint die psychische Belastung des Kollektivs im Follow-up geringer als zum Zeitpunkt Baseline, der Unterschied ist jedoch nicht deutlich genug, um bei einer so kleinen Stichprobe signifikant zu werden.

Bei dem BDI erfolgt die Betrachtung des durchschnittlichen Summenwerts der Patientinnen. Dieser lag im Follow-up $(M=24,1)$ signifikant niedriger als bei der Baseline-Messung $(M=$ 29,0). Es kann somit davon ausgegangen werden, dass die Patientinnen im Follow-up im Durchschnitt weniger depressiv waren als zum Zeitpunkt Baseline.

Auch die drei Skalen der IES-R wiesen im Follow-up deskriptiv niedrigere Werte als bei der Baseline-Messung auf. Diese Verbesserung ist bei den Skalen Intrusion und Übererregung 
sehr geringfügig, die Skala Vermeidung besserte sich dagegen signifikant im Wilcoxon-Vorzeichen-Rang-Test. Der FDS verzeichnete ebenfalls in allen Skalen niedrigere Werte im Follow-up. Diese Unterschiede sind jedoch alle sehr geringfügig und nicht signifikant. Es ist somit von einem im Mittel unveränderten Befund auszugehen.

Die Ergebnisse des BPI ergeben hingegen kein einheitliches Bild: Zum einen zeigt die Skala Primitive Abwehr und Objektbezogenheit im Wilcoxon-Vorzeichen-Rang-Test einen signifikanten Unterschied zwischen den beiden Messpunkten. Im Follow-up fand sich der niedrigere Median, sodass bei dieser Skala von einer Besserung auszugehen ist. Auch der BPI-Cut20, ein globaler Kennwert, war gegenüber der Baseline-Messung im Follow-up gesunken, jedoch nicht in signifikantem Ausmaß. Dem gegenüber stehen drei Skalen, die im Follow-up deskriptiv höhere Werte erreichten und einen eindeutig positiven Trend des BPI-Verlaufs unwahrscheinlich machen.

Bei der BSL überwiegen deutlich die Skalen, die im Follow-up numerisch höhere Werte als bei der Baseline-Messung aufwiesen. Die Symptomlast ist somit nicht gesunken, sondern eher angestiegen. Da die Unterschiede geringfügig und statistisch nicht signifikant sind, kann insgesamt von einer unveränderten Symptomlast gesprochen werden.

Hypothese 2 kann somit nur zum kleineren Teil angenommen werden.

\subsubsection{Deskriptiver Vergleich der TFM-Daten über die Phasen}

Die Abbildungen 12 bis 16 geben einen graphischen Überblick über die fünf Parameter, die für die Analysen ausgewählt wurden (s. Kap. 3.2.2.3), in den verschiedenen Messphasen der Baseline- und der Follow-up-Untersuchung. Zusätzlich ist zum Vergleich mit einer gesunden Stichprobe jeweils der Graph der Kontrollgruppe aus der Baseline-Untersuchung ergänzt. Die TFM-Daten der Kontrollgruppe wurden bereits von Bornschein (2014) berichtet und diskutiert. In die Baseline- und Follow-up-Graphen sind alle Patientinnen aus dem Followup-Kollektiv miteinbezogen - auch wenn einzelne Phasenmittelwerte von ihnen fehlen (z. B. durch Abbruch der Untersuchung). Soweit kein Einfluss auf die restlichen Phasen bestand, war ein Grund für einen Ausschluss aus der deskriptiven Auswertung der übrigen Messung nicht gegeben. Auch Patientin E1 blieb aus diesem Grund bezüglich der Graphen eingeschlossen. Hierdurch kann es jedoch zu leichten Unterschieden in den Mittelwerten und Standardabweichungen in Bezug auf die Kapitel 4.2.4, 4.3.4, 4.3.5 und 4.3.6 mit den ANOVA-Berechnungen und abhängigen t-Tests kommen. 


\subsubsection{Die Herzrate}

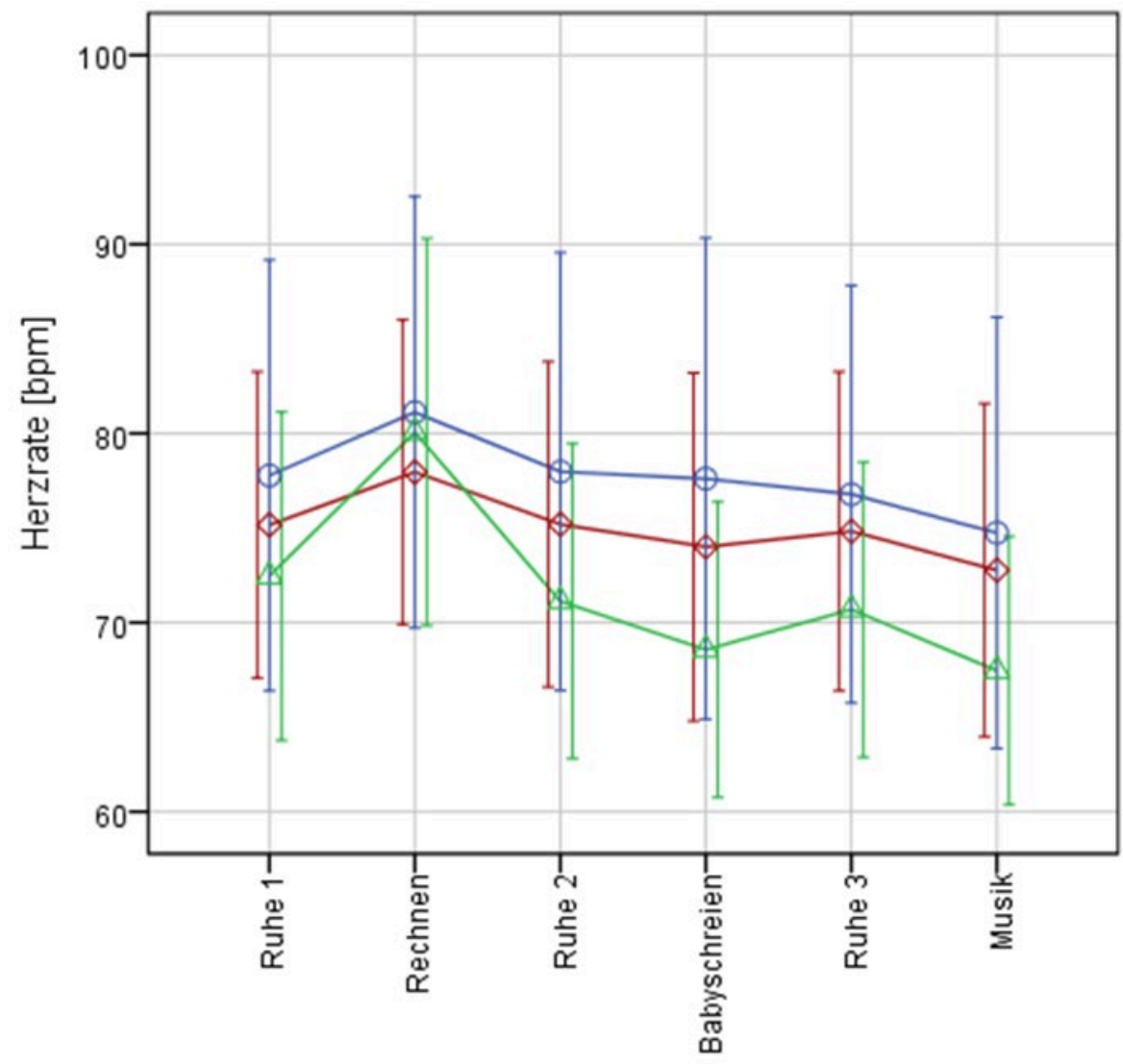

Baseline

$\diamond$ Follow-up

$\triangle$ Kontrollgruppe

Messphasen

Abbildung 12: HR in bpm über die verschiedenen Messphasen, dargestellt sind die Phasenmittelwerte mit Fehlerbalken (+/- 1 Standardabweichung)

Im Follow-up wurde zu Beginn der Messung (Ruhe 1) eine durchschnittliche Herzrate von $75,2$ ( $S D=8,1) \mathrm{bpm}$ aufgezeichnet. Die höchste mittlere Herzrate wurde in der Stressphase Rechnen mit 78,0 (SD = 8,1) bpm erreicht. Während in Ruhe 2 und Ruhe 3 Herzraten etwa in der Höhe von Ruhe 1 zu verzeichnen waren, war die Herzrate in den Phasen Babyschreien und Musik gegenüber der Ruhe 1-Phase abgesunken. Bei der Entspannungsmusik wurde mit $72,8(S D=8,8)$ bpm der niedrigste Wert erreicht. Der Herzraten-Graph der Baseline-Untersuchung verläuft ähnlich wie der Graph der Follow-up-Untersuchung, jedoch jeweils mit einer in jeder Phase durchschnittlich um 2,7 bpm höheren Herzrate. In der BaselineUntersuchung zeigte sich in der Phase Babyschreien im Gegensatz zum Follow-up kein Absinken der Herzrate gegenüber den Ruhephasen. Die Kontrollgruppe dagegen hatte eine deutlich höhere Schwankungsbreite der Herzrate im Verlauf der Untersuchung. In der Phase Rechnen fand sich ein erheblich höherer Anstieg der Herzrate gegenüber Ruhe 1, als es bei der Baseline- und Follow-up-Untersuchung der Fall war. Auch das Absinken der Herzrate während der Phase Babyschreien und Entspannungsmusik war deutlicher als bei den Patientinnen in der Follow-up-Untersuchung. Im Vergleich zur Ruhe 1-Phase lag die durchschnittliche Herzrate der Kontrollgruppe in Ruhe 2 und 3 etwas niedriger. 


\subsubsection{Der systolische Blutdruck}

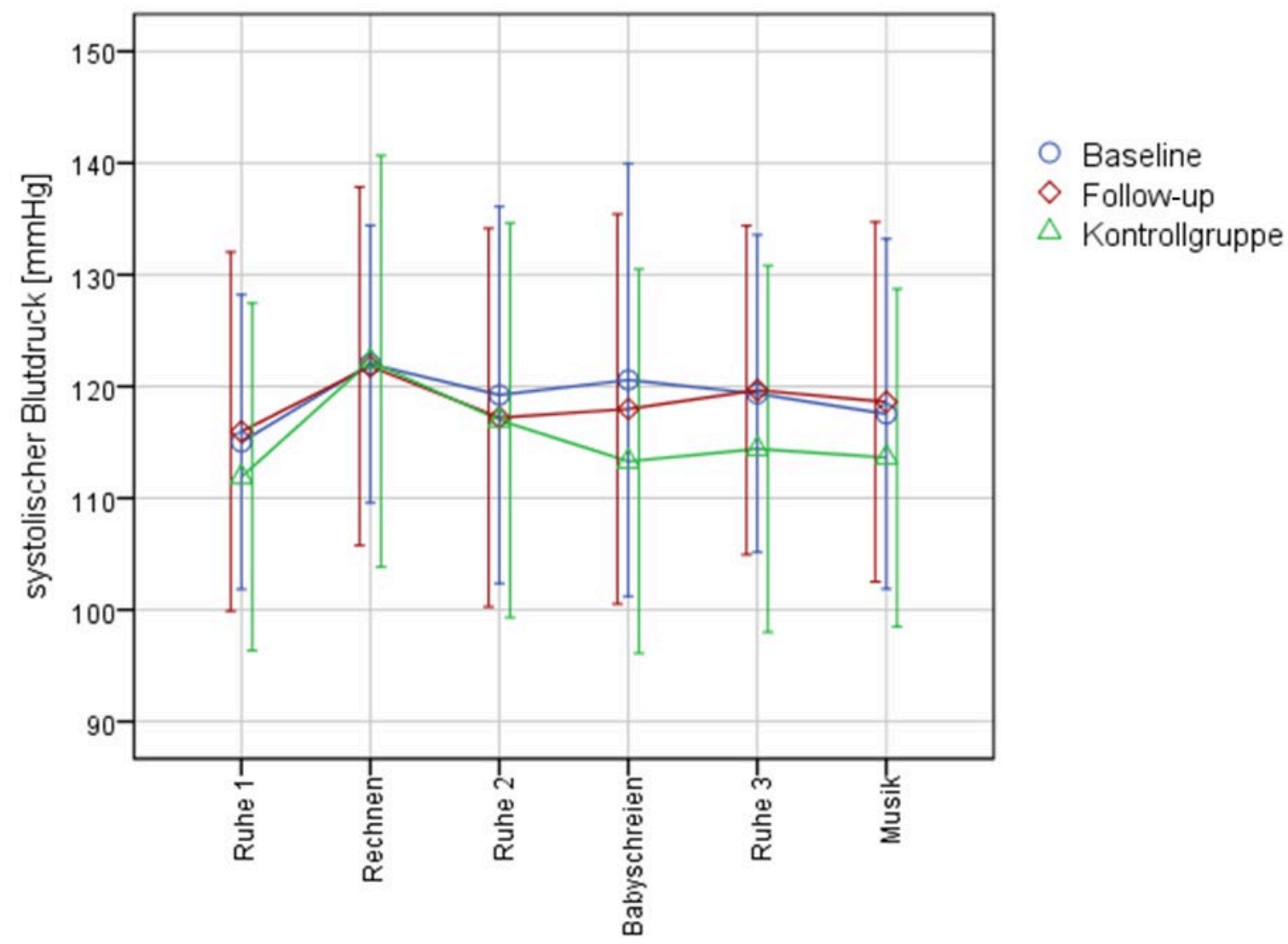

Messphasen

Abbildung 13: sBP in $\mathrm{mmHg}$ über die verschiedenen Messphasen, dargestellt sind die Phasenmittelwerte mit Fehlerbalken (+/- 1 Standardabweichung)

Der systolische Blutdruck der Follow-up-Kohorte war in Ruhe 1 mit 116,0 (SD = 16,1) mmHg am niedrigsten und stieg in der nachfolgenden Phase Rechnen auf seinen höchsten Wert mit 121,8 (SD = 16,0) $\mathrm{mmHg}$ an. In Ruhe 2 war er wiederum etwas abgesunken und zeigte sich nun über die restlichen Phasen relativ konstant und mit einem leichten Anstieg zur Phase Ruhe 3 hin. Während der systolische Blutdruck bei der Baseline-Untersuchung in den ersten beiden sowie in den letzten beiden Phasen der Untersuchung weitgehend identisch mit dem der Follow-up-Untersuchung war, lag er in Ruhe 2 und Babyschreien etwas über dem Blutdruck im Follow-up. Die Kontrollgruppe zeigte wie schon bei der Herzrate eine höhere Schwankungsbreite, die jedoch vor allem in der Phase Babyschreien hervorstach. Hier erfolgte ein Absinken des Blutdrucks fast bis auf das Niveau der Ruhe 1-Phase. Auch in den beiden nachfolgenden Phasen (Ruhe 3 und Entspannungsmusik) blieb der Blutdruck weitgehend so niedrig. 
4.3.3.3 Der totale periphere Widerstandsindex

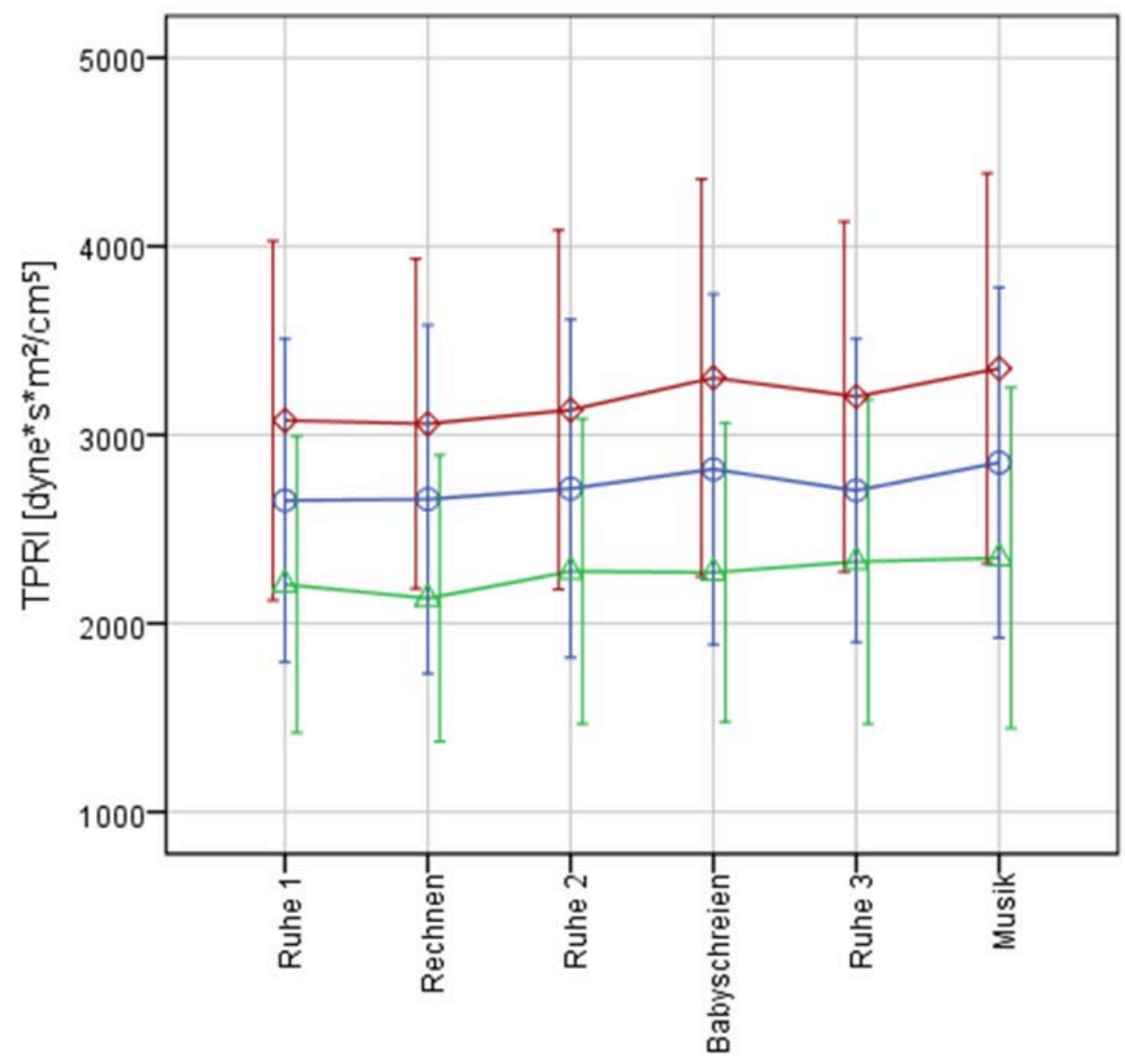

Baseline

$\diamond$ Follow-up

$\triangle$ Kontrollgruppe

Messphasen

Abbildung 14: TPRI in dyn* $\mathrm{s}^{*} \mathrm{~m}^{2} / \mathrm{cm}^{5}$ über die verschiedenen Messphasen, dargestellt sind die Phasenmittelwerte mit Fehlerbalken (+/- 1 Standardabweichung)

Insgesamt zeigte sich der TPRI im Verlauf der Untersuchung als relativ konstanter Parameter. Im Follow-up waren die höchsten Werten mit 3302,6 (SD = 1053,7) und 3352,2 $(S D=1036,0)$ dyn* $\mathrm{s}^{*} \mathrm{~m}^{2} / \mathrm{cm}^{5}$ in den Phasen Babyschreien und Entspannungsmusik zu finden. Der niedrigste TPRI-Wert dagegen wurde in der Phase Rechnen verzeichnet, er ist mit $3058,8(S D=874,3) \mathrm{dyn}^{*} \mathrm{~s}^{*} \mathrm{~m}^{2} / \mathrm{cm}^{5}$ aber nur geringfügig niedriger als in Ruhe 1 und 2 . Der TPRI im Follow-up erreichte deutlich höhere Werte als bei der Baseline-Messung oder in der Kontrollgruppe. Im Schnitt lagen die Werte des Follow-ups 454 dyn*s* ${ }^{2} / \mathrm{cm}^{5}$ über der Baseline-Messung und $928 \mathrm{dyn}^{*} \mathrm{~s}^{*} \mathrm{~m}^{2} / \mathrm{cm}^{5}$ über der Kontrollgruppe. Die Kontrollgruppe hatte somit auch deutlich niedrigere TPRI-Werte als die Patientinnen in der Baseline-Messung. Der Verlauf ist bei allen drei Gruppen ähnlich, der Graph der Kontrollgruppe unterscheidet sich jedoch etwas von den Graphen der Baseline- und der Follow-up-Untersuchung. Bei der Kontrollgruppe sank in der Phase Rechnen der TPRI etwas deutlicher ab, dafür waren die TPRI-Werte von Ruhe 2 bis zum Ende der Untersuchung weitgehend konstant. Dies bedeutet, dass im Gegensatz zu den anderen beiden Graphen kein Anstieg des TPRI während des Babyschreiens und der Entspannungsmusik zu erkennen ist. 
4.3.3.4 Die Präejektionsperiode

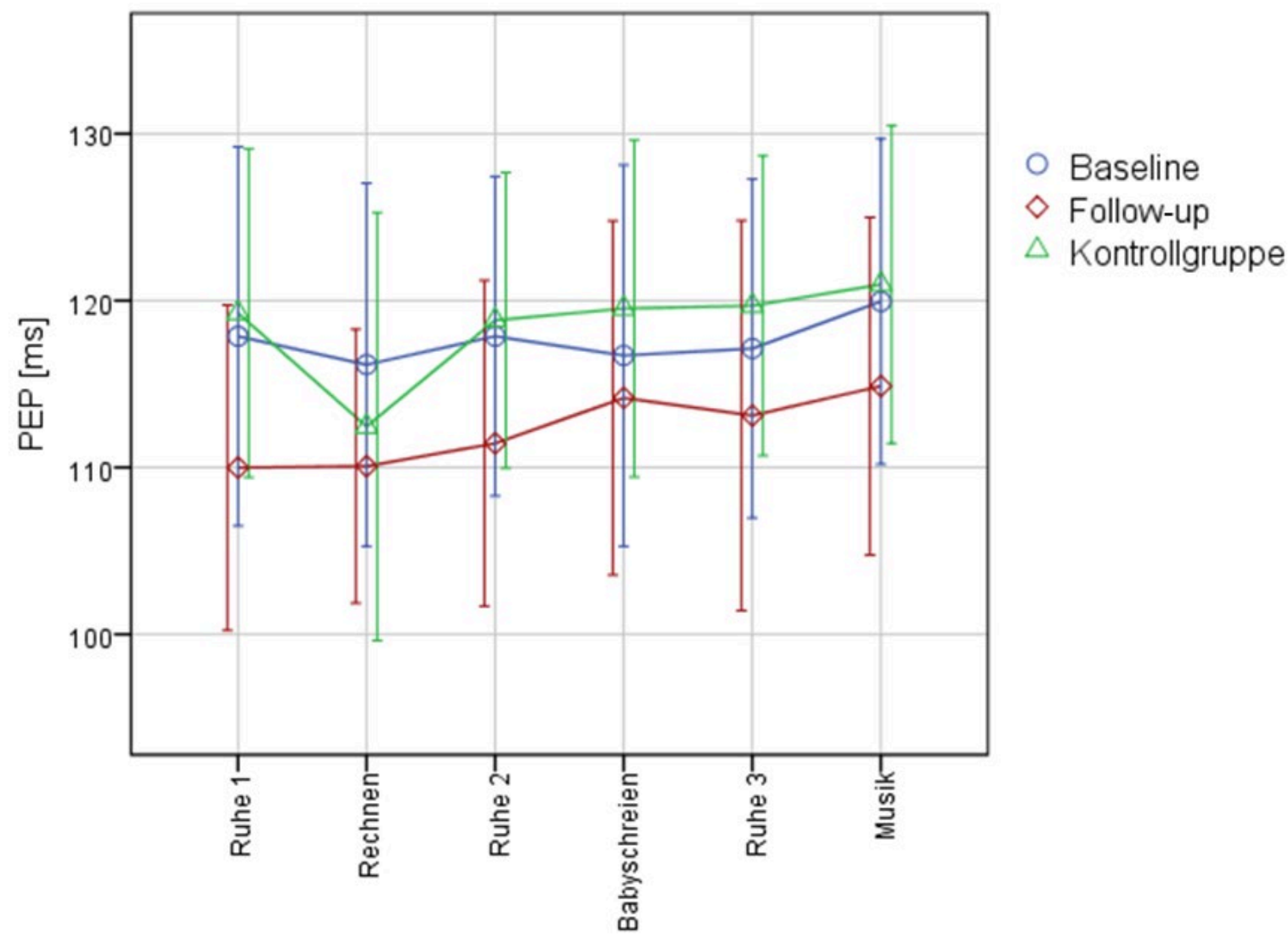

Messphasen

Abbildung 15: PEP in ms über die verschiedenen Messphasen, dargestellt sind die Phasenmittelwerte mit Fehlerbalken (+/- 1 Standardabweichung)

Die niedrigsten Werte der PEP wurden in den ersten beiden Phasen der Untersuchung aufgezeichnet, in Ruhe 2 erfolgte ein leichter Anstieg. Während des Babyschreiens und der Entspannungsmusik waren die höchsten Werte der PEP aufgetreten, mit einem zwischenzeitlichen Absinken während der Ruhe 3. Sowohl der Baseline-Graph als auch der Graph der Kontrollgruppe zeigen einen deutlich anderen Verlauf und erreichen in allen Phasen höhere PEP-Werte als im Follow-up. Der Baseline-Graph hat insgesamt wenige Ausschläge. Sein höchster Wert findet sich bei der Entspannungsmusik, wo ein deutlicher Anstieg der PEP gegenüber den anderen Phasen zu erkennen ist. In der Rechen- und in der Babyschreiphase sank die PEP dagegen geringfügig ab. In dem Graph der Kontrollgruppe fällt vor allem der starke Abfall der PEP während der Rechenphase ins Auge. Alle anderen Phasen waren auf einem weitgehend konstanten PEP-Niveau, auch hier sind die durchschnittlich höchsten PEP-Werte während der Entspannungsmusik aufgezeichnet worden. 


\subsubsection{Die Baroreflexsensitivität}

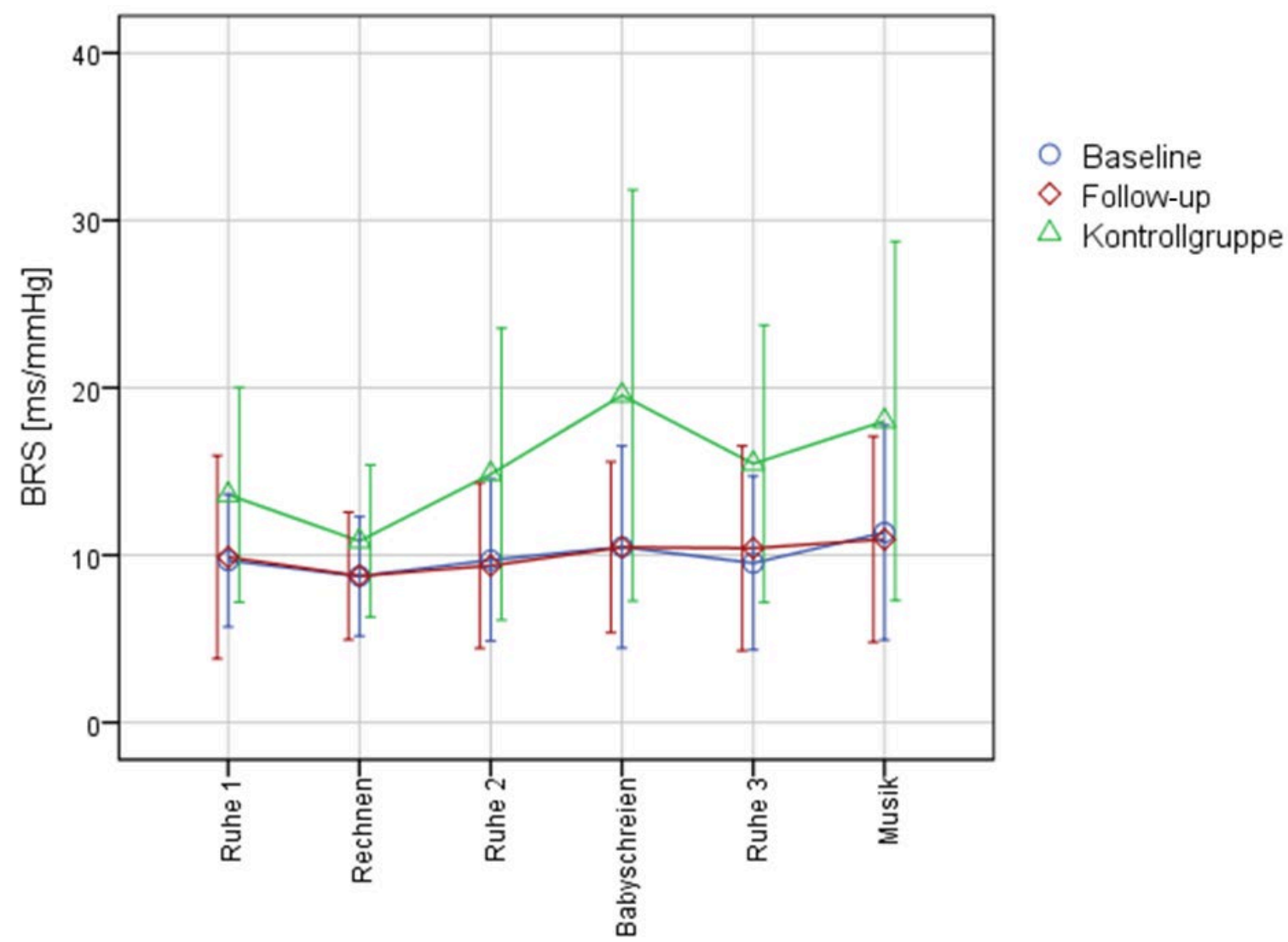

Messphasen

Abbildung 16: BRS in ms/mmHg über die verschiedenen Messphasen, dargestellt sind die Phasenmittelwerte mit Fehlerbalken (+/-1Standardabweichung)

Im Follow-up war die BRS über die Phasen weitgehend ohne größere Veränderungen. Sie war gegen Ende der Messung leicht höher als zu Beginn. In der Rechenphase sank die BRS geringfügig ab, stieg in Ruhe 2 jedoch wieder auf das Niveau von Ruhe 1 an $(M=9,9, S D=$ 6,1 ms/mmHg). Der Baseline-Graph ist fast identisch mit dem Graphen des Follow-ups. Einzig in der Phase Ruhe 3 zeigt sich eine Abweichung: Die BRS verringerte sich hier bei der Baseline-Messung leicht, während sie bei dem Follow-up gegenüber der vorigen Phase konstant war. Der Graph der Kontrollgruppe ist durch eine deutlich höhere Schwankungsbreite der BRS gekennzeichnet, ähnlich wie es auch schon bei der Herzrate festgestellt wurde. Zudem liegen die Werte in allen Phasen teilweise deutlich über den Messwerten der Patientinnen. In der Phase Rechnen ist ein starker Abfall und in der Phase Babyschreien ein noch stärkerer Anstieg der BRS zu beschreiben. Jeweils in den Ruhephasen nach den Stressphasen näherte sich die BRS wieder ihrem Ruhe-Niveau (wie in Ruhe 1) an. Auch bei der Kontrollgruppe waren die BRS-Werte am Ende der Untersuchung höher als zu Beginn, die höchsten Werte der BRS wurden jedoch hier während des Babyschreiens erreicht, ein Anstieg, der bei dem Baseline- und Follow-up-Graphen der Patientinnen völlig ausblieb. 


\subsubsection{Kardiovaskuläre Ruheaktivierung zu den Messzeitpunkten (Hypothese 3a)}

Für einen isolierten Vergleich von Baseline und Follow-up bzgl. des kardiovaskulären Systems in Ruhebedingungen wurden die in Kapitel 3.2.2.3 festgelegten Parameter mit einem abhängigen t-Test auf Unterschiede zwischen Ruhe 1 Baseline und Ruhe 1 Follow-up getestet. Einzig für die in Ruhe 1 nicht normalverteilte BRS wurde ein Wilcoxon-VorzeichenRang-Test verwendet. Es wurde die Phase Ruhe 1 ausgewählt, da eine Betrachtung der kardiovaskulären Ruheaktivierung zu Beginn der Messung und insbesondere ohne jeglichen Einfluss durch die Stressphasen oder die Recovery-Reaktion erfolgen soll.

Im Follow-up war die HR entsprechend der Hypothese 3a während der Phase Ruhe 1 im Mittel um 3,6 bpm niedriger als in der Baseline-Untersuchung. Dieser Unterschied der beiden Messzeitpunkte wurde im abhängigen t-Test signifikant $(t(24)=1,8 ; p$ (einseitig) $<0,05$ ). Gegenüber der Baseline-Untersuchung war im Follow-up der systolische Blutdruck während der Phase Ruhe 1 im Mittel geringfügig um 0,8 mmHg gestiegen. Damit veränderte sich der systolische Blutdruck entgegen der Hypothese 3a. Auch bei zweiseitiger explorativer Testung erreichte diese Zunahme jedoch keine Signifikanz im abhängigen t-Test $(t(24)=-0,2$; $p$ (zweiseitig $)=0,83)$. Der TPRI, der von Ruhe 1 Baseline zu Ruhe 1 Follow-up im Mittel einen Anstieg von 2652 auf 3038 dyn*s* ${ }^{2} / \mathrm{cm}^{5}$ verzeichnete, verhielt sich ebenfalls gegensinnig zu der Hypothese 3a, in der ein Absinken des systolischen Blutdrucks und des TPRI im Follow-up erwartet worden war. Dieser Anstieg des TPRI zeigte sich jedoch bei zweiseitiger explorativer Testung signifikant $(t(24)=-2,3$; $p$ (zweiseitig) $<0,05)$. Der Phasenmittelwert der PEP in Ruhe 1 war mit $110 \mathrm{~ms}$ bei dem Follow-up niedriger als in der Baseline-Messung (117 ms), welches erneut einer zur Hypothese 3a gegenläufigen, in diesem Fall jedoch nicht einer zweiseitig signifikanten Reaktion entspricht $(t(21)=1,9 ; p$ (zweiseitig) $=0,08)$. Zuletzt erfolgte der Wilcoxon-Vorzeichen-Rang-Test bei der BRS. Während sich in der Baseline-Messung ein Median von 9,2 ms/mmHg ergab, war dieser im Follow-up mit $8,3 \mathrm{~ms} / \mathrm{mmHg}$ niedriger. Dies ist, wie sich gleichzeitig an den Rängen abzeichnete, eine Veränderung entgegen der Hypothese 3a, in der für PEP und BRS ein Anstieg unter Ruhebedingungen zum Follow-up vermutet worden war. In der explorativen zweiseitigen Testung wurde keine Signifikanz erreicht $(z=-0,5, p$ (zweiseitig) $=0,65)$.

\subsubsection{Reactivity: Aktivierung durch die Stressoren zu den Messzeitpunkten}

Mit zwei 2 x 2 ANOVAs für Messwiederholungen soll die Wirkung der Stressoren (Rechnen bzw. Babyschreien gegenüber Ruhe 1) sowie der Zeit (Baseline vs. Follow-up) auf die fünf in Kapitel 3.2.2.3 festgelegten Parameter untersucht werden (s. Tab. 15 und 16). Es wird somit die Aktivierung durch die Stressoren, die „Reactivity“, betrachtet. 
Tabelle 15: Reactivity Rechnen. Phasenmittelwerte und Standardabweichungen der Phasen Ruhe 1 und Rechnen (jeweils für Baseline und Follow-up) und eine 2 × 2 ANOVA für Messwiederholungen für die Parameter HR in [bpm], sBP in [mmHg], TPRI in [dyn* $\left.{ }^{*} \mathrm{~m}^{2} / \mathrm{cm}^{5}\right]$, PEP in [ms] und BRS in [ms/mmHg]. Die Berechnungen erfolgten unter Ausschluss der Patientin E1 (s. Kap. 4.2.5).

\begin{tabular}{|c|c|c|c|c|c|c|c|}
\hline & & & $\begin{array}{c}\text { HR } \\
(N=24)\end{array}$ & $\begin{array}{c}\mathbf{s B P} \\
(N=24)\end{array}$ & $\begin{array}{c}\text { TPRI } \\
(N=24)\end{array}$ & $\begin{array}{c}\text { PEP } \\
(N=22)\end{array}$ & $\begin{array}{c}\text { BRS } \\
(N=24)\end{array}$ \\
\hline \multicolumn{8}{|c|}{ Mittelwerte mit Standardabweichung über die Phasen } \\
\hline \multirow{4}{*}{ Ruhe 1} & \multirow{2}{*}{ Baseline } & $M$ & 76,9 & 115,5 & 2676,8 & 117,2 & 9,9 \\
\hline & & $S D$ & 10,7 & 13,2 & 866,7 & 11,9 & 3,9 \\
\hline & \multirow{2}{*}{$\begin{array}{l}\text { Follow- } \\
\text { up }\end{array}$} & $M$ & 74,3 & 116,7 & 3101,0 & 110,3 & 10,3 \\
\hline & & $S D$ & 6,5 & 16,1 & 919,0 & 9,9 & 6,1 \\
\hline \multirow{4}{*}{ Rechnen } & \multirow{2}{*}{ Baseline } & $M$ & 80,3 & 121,7 & 2660,4 & 115,5 & 8,8 \\
\hline & & $S D$ & 10,9 & 12,6 & 943,3 & 11,5 & 3,6 \\
\hline & \multirow{2}{*}{$\begin{array}{c}\text { Follow- } \\
\text { up }\end{array}$} & $M$ & 77,0 & 122,7 & 3080,3 & 110,3 & 9,1 \\
\hline & & $S D$ & 7,1 & 16,0 & 823,9 & 8,3 & 3,7 \\
\hline \multicolumn{8}{|c|}{2 x 2 ANOVA für Messwiederholung zur Reactivity Rechnen } \\
\hline \multirow{3}{*}{ Messzeitpunkt } & \multicolumn{2}{|c|}{ Teststatistik $F$} & 2,7 & 0,1 & 6,4 & 3,1 & 0,1 \\
\hline & \multicolumn{2}{|c|}{ Freiheitsgrade } & $1 ; 23$ & $1 ; 23$ & $1 ; 23$ & $1 ; 21$ & $1 ; 23$ \\
\hline & \multicolumn{2}{|c|}{ Signifikanz } & 0,12 & 0,74 & $<0,05$ & 0,09 & 0,74 \\
\hline \multirow{3}{*}{$\begin{array}{l}\text { Stressor } \\
\text { Rechnen }\end{array}$} & \multicolumn{2}{|c|}{ Teststatistik $F$} & 48,6 & 31,9 & 0,2 & 0,9 & 7,1 \\
\hline & \multicolumn{2}{|c|}{ Freiheitsgrade } & $1 ; 23$ & $1 ; 23$ & $1 ; 23$ & $1 ; 21$ & $\begin{array}{l}1 ; \\
23\end{array}$ \\
\hline & \multicolumn{2}{|c|}{ Signifikanz } & $<0,001$ & $<0,001$ & 0,65 & 0,36 & $<0,05$ \\
\hline \multirow{3}{*}{$\begin{array}{c}\text { Wechsel- } \\
\text { wirkung } \\
\text { Messzeitpunkt } \\
\text { x Stressor }\end{array}$} & \multicolumn{2}{|c|}{ Teststatistik $F$} & 1,4 & 0,01 & 0,003 & 1,1 & 0,01 \\
\hline & \multicolumn{2}{|c|}{ Freiheitsgrade } & $1 ; 23$ & $1 ; 23$ & $1 ; 23$ & $1 ; 21$ & $1 ; 23$ \\
\hline & \multicolumn{2}{|c|}{ Signifikanz } & 0,26 & 0,92 & 0,95 & 0,30 & 0,92 \\
\hline
\end{tabular}

Die Berechnungen erfolgten unter Ausschluss der Patientin E1 (s. Kap. 4.2.5). Daher weichen die hier berichteten Mittelwerte und Standardabweichungen der Phase Ruhe 1 von den in den Kapiteln 4.2.4 und 4.3.4 aufgeführten Ergebnissen leicht ab. Für einen Vergleich befinden sich im Anhang die Ergebnisse der gleichen Berechnungen unter Einschluss der Patientin E1. Da die TPRI-Werte einer Patientin in der Phase Babyschreien fehlen (s. Kap. 4.1.1), unterscheiden sich die Tabellen 17 und 18 bzgl. der Mittelwerte und Standardabweichungen des TPRI. 
Die Phase Rechnen führte bei den Parametern HR, sBP und BRS im Vergleich zur Phase Ruhe 1 zu signifikanten bis höchstsignifikanten Veränderungen, gleichzeitig war jedoch einzig für den Parameter TPRI ein signifikanter Zeiteffekt zu verzeichnen. Dieser Zeiteffekt findet sich auch in der ANOVA zur Reactivity des Babyschreiens und ergibt sich aus den durchschnittlich höheren Werten des TPRI im Follow-up. Das Babyschreien hatte verglichen mit der Phase Ruhe 1 lediglich einen signifikanten Einfluss auf den TRPI. Bei der PEP sind jedoch signifikante Wechselwirkungen zwischen Zeit und Phase zu beschreiben.

Tabelle 16: Reactivity Babyschreien. Phasenmittelwerte und Standardabweichungen der Phasen Ruhe 1 und Babyschreien (jeweils für Baseline und Follow-up) und eine 2 × 2 ANOVA für Messwiederholungen für die Parameter HR in $[\mathrm{bpm}], \mathrm{sBP}$ in $[\mathrm{mmHg}]$, TPRI in $\left[\mathrm{dyn}^{*} \mathrm{~s}^{*} \mathrm{~m}^{2} / \mathrm{cm}^{5}\right]$, PEP in $[\mathrm{ms}]$ und BRS in [ms $/ \mathrm{mmHg}$. Die Berechnungen erfolgten unter Ausschluss der Patientin E1 (s. Kap. 4.2.5).

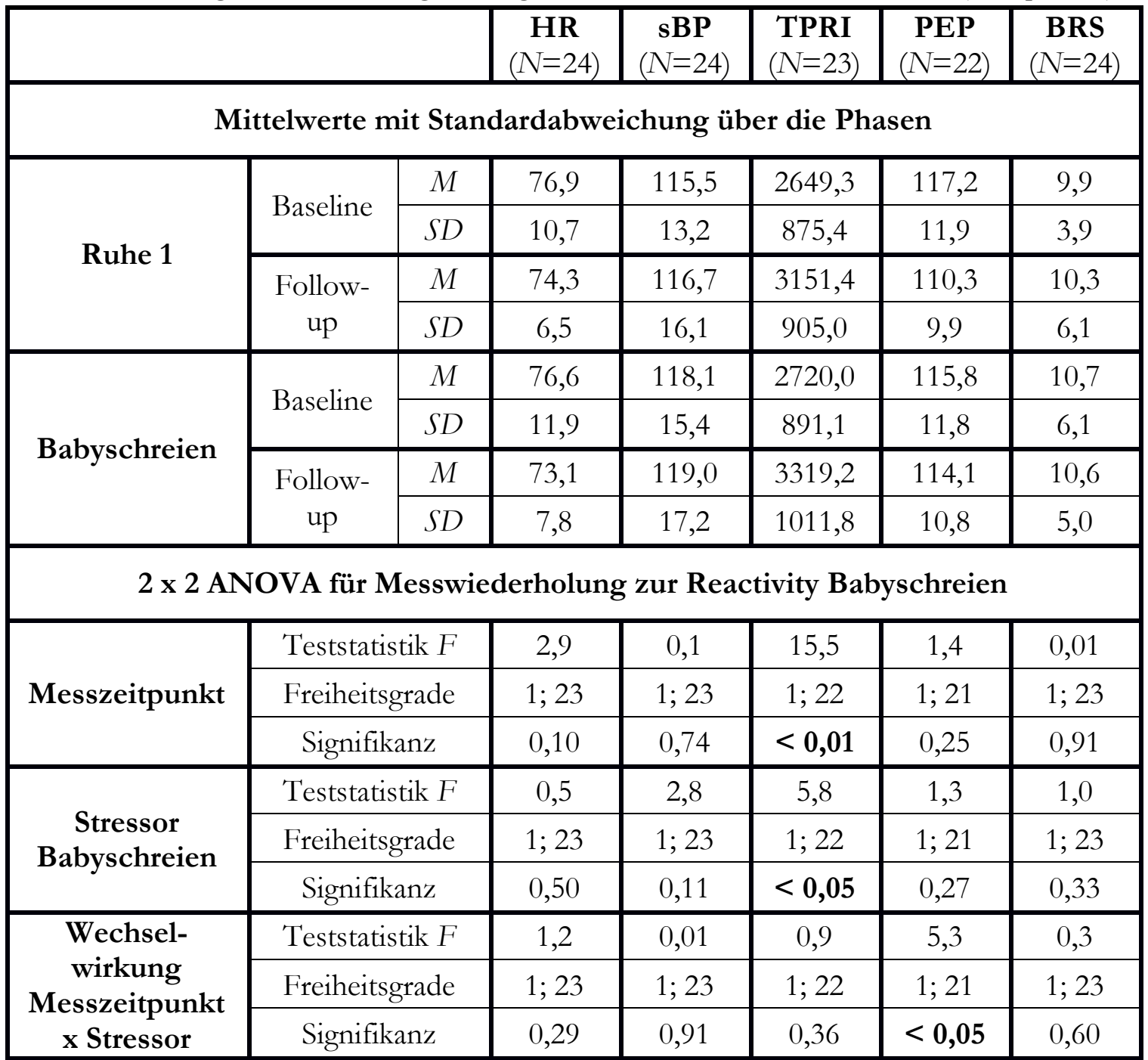




\subsubsection{Recovery: Erholung nach der Stressreaktion zu den Messzeitpunkten}

Nach der Reactivity soll nun das Augenmerk auf der Recovery liegen, die sich an die Stressreaktion anschließt. Hierzu soll mithilfe von zwei 2 × 2 ANOVAs für Messwiederholungen der Einfluss der Recovery (Rechnen bzw. Babyschreien gegenüber Entspannungsmusik) sowie der Zeit (Baseline vs. Follow-up) auf die fünf in Kapitel 3.2.2.3 festgelegten Parameter betrachtet werden (s. Tab. 17 und 18).

Tabelle 17: Recovery Rechnen. Phasenmittelwerte und Standardabweichungen der Phasen Rechnen und Musik (jeweils für Baseline und Follow-up) und eine $2 \times 2$ ANOVA für Messwiederholungen für die Parameter HR in [bqm], sBP in [mmHg], TPRI in [dyn*s* $\mathrm{m}^{2} / \mathrm{cm}^{5}$ ], PEP in [ms] und BRS in $[\mathrm{ms} / \mathrm{mmHg}$. Die Berechnungen erfolgten unter Ausschluss der Patientin E1 (s. Kap. 4.2.5).

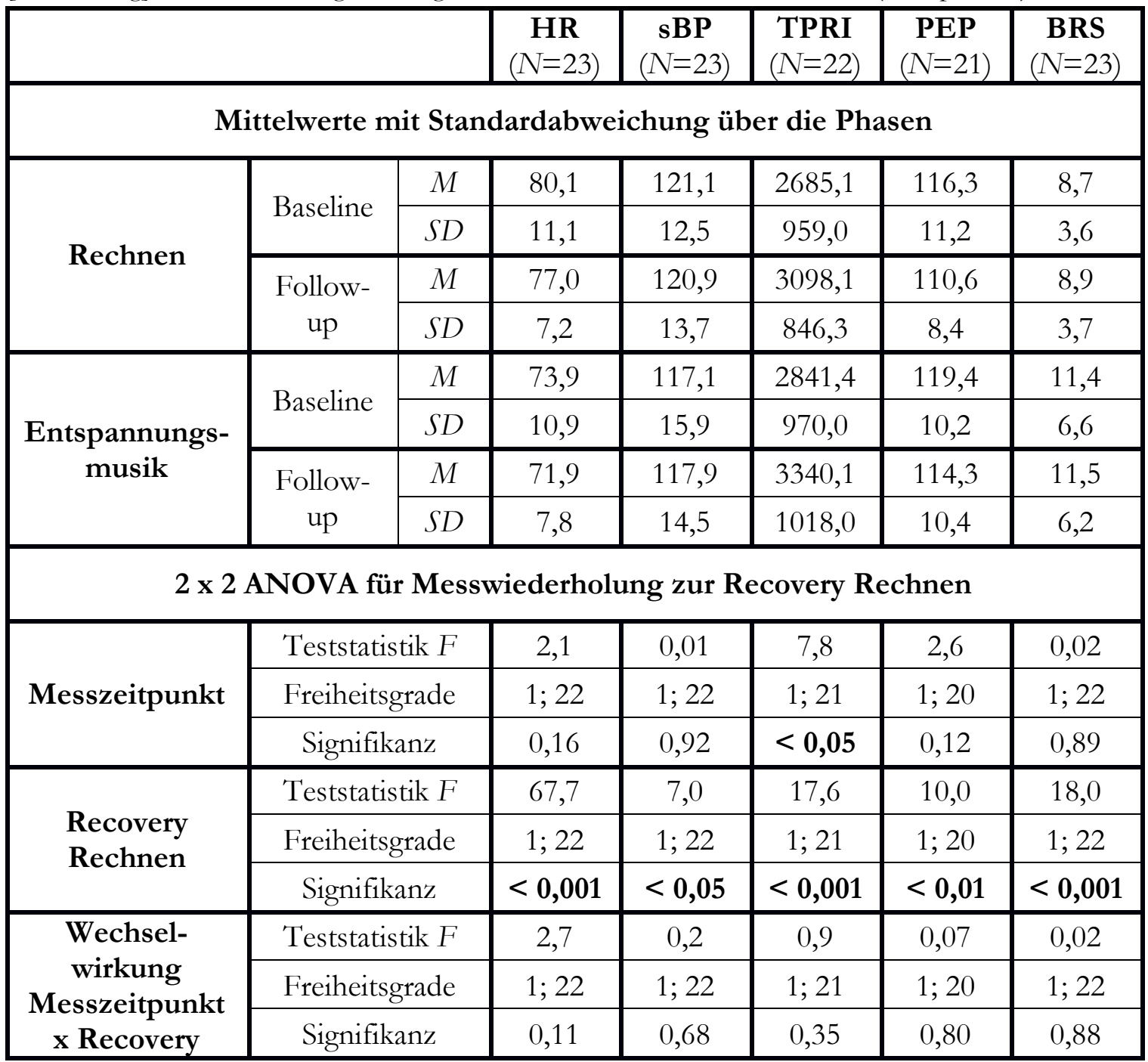

Auch für diese Berechnungen wurde die Patientin E1 ausgeschlossen (s. Kap. 4.2.5). Daher kann es auch hier zu Abweichungen bezüglich der Mittelwerte und Standardabweichungen der Phase Musik im Vergleich zu den im Kapitel 4.2.4 aufgeführten Ergebnissen kommen. Eine Vergleichstabelle mit allen Rechnungen unter Einbeziehung der Patientin E1 im Anhang dient wiederum einer Evaluation des Ausschlusses der Patientin E1. Da zudem eine Patientin die Messung vor der Phase Musik in der Baseline-Messung abbrach und bei einer 
weiteren Patient die TPRI-Werte in den Phasen Babyschreien und Musik fehlten (s. Kap. 4.1.1), ist die Anzahl der in die beiden Recovery-ANOVAs eingehenden Fälle im Vergleich zu Kapitel 4.3.5 reduziert. Somit ergeben sich auch gegenüber Kapitel 4.3.5 abweichende Mittelwerte und Standardabweichungen der Phasen Rechnen und Babyschreien.

Bei der Betrachtung der beiden Stressoren fand sich nur bei dem TPRI ein signifikanter bis hochsignifikanter Zeiteffekt, der wie schon bei der Reactivity durch durchschnittlich höhere TPRI-Werte im Follow-up gekennzeichnet war. Bemerkenswert ist, dass sich die Phasen Rechnen und Musik in allen fünf betrachteten Parametern signifikant bis höchstsignifikant unterschieden, während die Phase Babyschreien in keinem einzigen Parameter signifikant von der Phase Musik abwich. Signifikante Wechselwirkungen zwischen Messzeitpunkt und Recoverywirkung fanden sich bei beiden Stressoren nicht.

Tabelle 18: Recovery Babyschreien. Phasenmittelwerte und Standardabweichungen der Phasen Babyschreien und Musik (jeweils für Baseline und Follow-up) und eine 2 × 2 ANOVA für Messwiederholungen für die Parameter HR in [bqm], $\mathrm{sBP}$ in $\left[\mathrm{mmHg}\right.$, TPRI in $\left[\mathrm{dyn}^{*} \mathrm{~s}^{*} \mathrm{~m}^{2} / \mathrm{cm}^{5}\right.$ ], PEP in [ms] und BRS in [ms/mmHg]. Die Berechnungen erfolgten unter Ausschluss der Patientin E1 (s. Kap. 4.2.5).

\begin{tabular}{|c|c|c|c|c|c|c|c|}
\hline & & & $\begin{array}{c}\text { HR } \\
(N=23)\end{array}$ & $\begin{array}{c}\mathbf{s B P} \\
(N=23)\end{array}$ & $\begin{array}{c}\text { TPRI } \\
(N=22) \\
\end{array}$ & $\begin{array}{c}\text { PEP } \\
(N=21)\end{array}$ & $\begin{array}{c}\text { BRS } \\
(N=23)\end{array}$ \\
\hline \multicolumn{8}{|c|}{ Mittelwerte mit Standardabweichung über die Phasen } \\
\hline \multirow{4}{*}{ Babyschreien } & \multirow{2}{*}{ Baseline } & M & 75,7 & 117,1 & 2763,8 & 116,6 & 10,6 \\
\hline & & $S D$ & 11,4 & 15,0 & 886,5 & 11,5 & 6,2 \\
\hline & \multirow{2}{*}{$\begin{array}{l}\text { Follow- } \\
\text { up }\end{array}$} & $M$ & 72,5 & 117,4 & 3328,2 & 113,9 & 10,7 \\
\hline & & $S D$ & 7,4 & 15,8 & 1034,6 & 11,1 & 5,1 \\
\hline \multirow{4}{*}{$\begin{array}{l}\text { Entspannungs- } \\
\text { musik }\end{array}$} & \multirow{2}{*}{ Baseline } & $M$ & 73,9 & 117,1 & 2841,4 & 119,4 & 11,4 \\
\hline & & $S D$ & 10,9 & 15,9 & 970,0 & 10,2 & 6,6 \\
\hline & \multirow{2}{*}{$\begin{array}{l}\text { Follow- } \\
\text { up }\end{array}$} & $M$ & 71,9 & 117,9 & 3340,1 & 114,3 & 11,5 \\
\hline & & $S D$ & 7,8 & 14,5 & 1018,0 & 10,4 & 6,2 \\
\hline \multicolumn{8}{|c|}{$2 \times 2$ ANOVA für Messwiederholung zur Recovery Babyschreien } \\
\hline \multirow{3}{*}{ Messzeitpunkt } & \multicolumn{2}{|c|}{ Teststatistik F } & 2,2 & 0,03 & 12,6 & 1,1 & 0,002 \\
\hline & \multicolumn{2}{|c|}{ Freiheitsgrade } & $1 ; 22$ & $1 ; 22$ & $1 ; 21$ & $1 ; 20$ & $1 ; 22$ \\
\hline & \multicolumn{2}{|c|}{ Signifikanz } & 0,16 & 0,87 & $<0,01$ & 0,30 & 0,96 \\
\hline \multirow{3}{*}{$\begin{array}{c}\text { Recovery } \\
\text { Babyschreien }\end{array}$} & \multicolumn{2}{|c|}{ Teststatistik F } & 3,1 & 0,03 & 1,3 & 2,8 & 3,8 \\
\hline & \multicolumn{2}{|c|}{ Freiheitsgrade } & $1 ; 22$ & $1 ; 22$ & $1 ; 21$ & $1 ; 20$ & $1 ; 22$ \\
\hline & \multicolumn{2}{|c|}{ Signifikanz } & 0,09 & 0,87 & 0,26 & 0,11 & 0,06 \\
\hline \multirow{3}{*}{$\begin{array}{c}\text { Wechsel- } \\
\text { wirkung } \\
\text { Messzeitpunkt } \\
\text { x Recovery }\end{array}$} & \multicolumn{2}{|c|}{ Teststatistik $F$} & 3,3 & 0,1 & 0,6 & 2,3 & 0,003 \\
\hline & \multicolumn{2}{|c|}{ Freiheitsgrade } & $1 ; 22$ & $1 ; 22$ & $1 ; 21$ & $1 ; 20$ & $1 ; 22$ \\
\hline & \multicolumn{2}{|c|}{ Signifikanz } & 0,08 & 0,81 & 0,44 & 0,15 & 0,96 \\
\hline
\end{tabular}




\subsection{Der Einfluss der Therapiedauer (Hypothese 6)}

Als Maß für die Therapie dienen zwei Variablen: die psychotherapeutische stationäre Behandlung auf der Station 9 des Asklepios Fachklinikums Göttingen in Tagen, im Folgenden „Therapiedauer Station 9“ genannt, sowie die gesamte psychiatrische bzw. psychotherapeutische stationäre und teilstationäre Behandlung in Tagen (inklusive Station 9 und Tagesklinik), im Folgenden „Therapiedauer gesamt“" genannt. Für diese beiden Variablen wurden zunächst Korrelationen mit den Differenzen des Stressempfindens (Baseline - Follow-up) für die vier erhobenen Zeitpunkte Ruhe 1, Rechnen, Babyschreien und Musik berechnet. Die Korrelationen wurden entsprechend der 6. Hypothese einseitig getestet. Demnach wurde betrachtet, ob die Patientinnen mit einer hohen Therapiedauer auch ein besonders starkes Absinken bei dem Stressempfinden von Baseline zu Follow-up, also eine besonders starke Verbesserung des Stressempfindens, verzeichneten. Dies würde sich in einer positiven Korrelation widerspiegeln. Hierbei fanden sich jedoch keine signifikanten einseitigen Korrelationen. Da die Stichprobe klein ist, sollen alle Ergebnisse mit $p<0,10$ aufgeführt werden, damit auch kleinere Effekte rein explorativ erfasst werden. Hier ist jedoch einzig die Korrelation zwischen der „Therapiedauer Station 9“ und der Differenz Ruhe $1(r=0,33 ; p=0,053)$ zu nennen.

Die beiden Variablen „Therapiedauer Station 9“ und „Therapiedauer gesamt“ wurden zudem auf Korrelationen mit den Differenzen der psychometrischen Fragebögen (Baseline Follow-up) getestet. Die Differenzen der psychometrischen Fragebögen wurden aus den jeweiligen Gesamtscores (GSI der SCL-90-R, BDI-Summenwert, IES-R Übererregung, IESR Vermeidung, IES-R Intrusion, BPI-Cut-20, BSL-Gesamtskala sowie FDS-Gesamtskala) bestimmt. Auch hier erfolgte die Signifikanzbestimmung im Sinne der 6. Hypothese einseitig, d. h. es wurde untersucht, ob Patientinnen mit hohen Therapiedauern eine besonders starke Reduktion der Belastung in den psychometrischen Fragebögen zwischen Baseline und Follow-up und damit eine Verbesserung des psychischen Befindens aufweisen. Dies würde wiederum einer positiven Korrelation entsprechen. Es korreliert jedoch einzig die "Therapiedauer gesamt“ signifikant mit der Differenz der IES-R-Skala Vermeidung $(r=0,41$; $p<0,05)$. All diese Ergebnisse sprechen gegen einen Zusammenhang zwischen einer hohen Therapiedauer und positiven Veränderungen bzgl. des Stressempfindens und der psychometrischen Fragebögen von Baseline zu Follow-up. Es muss jedoch vor einer Interpretation dieser Ergebnisse geklärt werden, ob die Patientinnen mit längerer stationärer Therapiedauer bestimmte Merkmale aufweisen, die die Ergebnisse aus den obigen Betrachtungen beeinflussen könnten. Daher wurden für die beiden Variablen zur Therapiedauer zusätzlich mit dem Stressempfinden bei der Baseline-Untersuchung (Ruhe 1, Rechnen, Babyschreien und Musik) sowie mit dem psychometrischen Befund der Baseline-Untersuchung (anhand von GSI, BDI-Summenwert, IES-R Intrusion, IES-R Vermeidung, IES-R Übererregung, BPICut-20, BSL-Gesamtskala sowie FDS-Gesamtskala) Korrelationen berechnet. Alle Ergebnisse aus dieser Berechnung mit $p<0,10$ werden in Tabelle 19 aufgeführt. Angegeben wird hier die zweiseitige Signifikanz, da es sich um eine explorative Analyse ohne gerichtete Hypothese handelt. 
Tabelle 19: Alle Korrelationen (mit $p<0,10$ ) der beiden Variablen „Therapiedauer Station 9“ (stationäre Therapie auf der Station 9 des Asklepios Fachklinikums Göttingen in Tagen) und „Therapiedauer gesamt" (gesamte stationäre und teilstationäre psychiatrische Therapie in Tagen, somit inklusive der Behandlungen auf der Station 9 und in der Tagesklinik) mit den Variablen zum Stressempfinden und zum psychometrischen Befund der Baseline-Untersuchung.

\begin{tabular}{|c|l|c|c|}
\hline \multicolumn{2}{|c|}{ Korrelationen zwischen den Variablen } & $\begin{array}{c}\text { Spearmans } \\
\text { rho }\end{array}$ & $\begin{array}{c}p \\
\text { (zweiseitig) }\end{array}$ \\
\hline \multirow{2}{*}{$\begin{array}{c}\text { "Therapiedauer } \\
\text { Station 9“ }\end{array}$} & Stress Ruhe 1 (Baseline) & 0,62 & $<0,01$ \\
\cline { 2 - 4 } & Stress Musik (Baseline) & 0,42 & $<0,05$ \\
\hline \multirow{4}{*}{$\begin{array}{c}\text { "Therapiedauer } \\
\text { gesamt“ }\end{array}$} & Stress Ruhe 1 (Baseline) & 0,47 & $<0,05$ \\
\cline { 2 - 4 } & Stress Musik (Baseline) & 0,37 & 0,07 \\
\cline { 2 - 4 } & GSI der SCL-90-R (Baseline) & 0,46 & $<0,05$ \\
\cline { 2 - 4 } & BDI Summenwert (Baseline) & 0,35 & 0,09 \\
\cline { 2 - 4 } & IES-R Skala Intrusion (Baseline) & 0,58 & $<0,01$ \\
\cline { 2 - 4 } & IES-R Skala Vermeidung (Baseline) & 0,54 & $<0,01$ \\
\cline { 2 - 4 } & BSL-Gesamtskala (Baseline) & 0,49 & $<0,05$ \\
\cline { 2 - 4 } & FDS-Gesamtskala (Baseline) & 0,42 & $<0,05$ \\
\hline
\end{tabular}

Am stärksten korreliert das Stressempfinden in Ruhe 1 (Baseline) und die „Therapiedauer Station 9“ $(r=0,62 ; p=0,001)$. Diese Korrelation wurde in der Abbildung 17 visualisiert. Es fällt auf, dass alle Korrelationen positiv sind: Patientinnen mit einem besonders hohen Stressempfinden bzw. mit besonders hohen und damit pathologischen Werten in den psychometrischen Fragebögen nahmen nach der Baseline-Untersuchung besonders lange eine stationäre bzw. teilstationäre Behandlung in Anspruch. Dieser Zusammenhang gilt für einen Großteil der psychometrischen Fragebögen sowie für das Stressempfinden in Ruhe 1 und bei Musik. Eine Korrelation zu dem Stressempfinden in den Stressphasen der Messung konnte nicht ermittelt werden. Betrachtet man nur spezifisch die Therapie auf der Station 9, nahmen an dieser vor allem Patientinnen länger teil, die ein hohes Stressempfinden in Ruhe 1 und Musik bei der Baseline-Messung zeigten, ein Zusammenhang zu einem besonders pathologischen psychometrischen Befund in der Baseline-Untersuchung konnte nicht gefunden werden.

Zur besseren Einordnung dieser Ergebnisse wurde mithilfe einer multivariaten Regression der Zusammenhang zwischen der Behandlungsdauer (auf Station 9 oder gesamt) und den Differenzwerten (Baseline - Follow-up) des Stressempfindens bzw. der psychometrischen Fragebögen unter Adjustierung der jeweiligen Baseline-Werte betrachtet. Hierfür wurden die Differenzwerte als abhängige Größe gesetzt, die Behandlungsdauer und die jeweils zugehörigen Baseline-Werte gingen als Prädiktoren in die Gleichung ein. Doch auch wenn der Effekt der Baseline-Werte auf diese Weise kontrolliert wurde, konnte bei keinem Fragebogen und keiner Stressphase ein positiver und signifikanter Zusammenhang zwischen der Behandlungsdauer und den Differenzwerten (des Stressempfindens oder der psychometrischen Fragebögen) ermittelt werden. Die Koeffizienten zu den Regressionsrechnungen sind im Anhang 7.3 aufgelistet. 
Ob die Therapiedauer Auswirkungen auf die Reagibilität des autonomen Stressregulationssystems hat, soll in Kapitel 4.5 genauer untersucht werden.

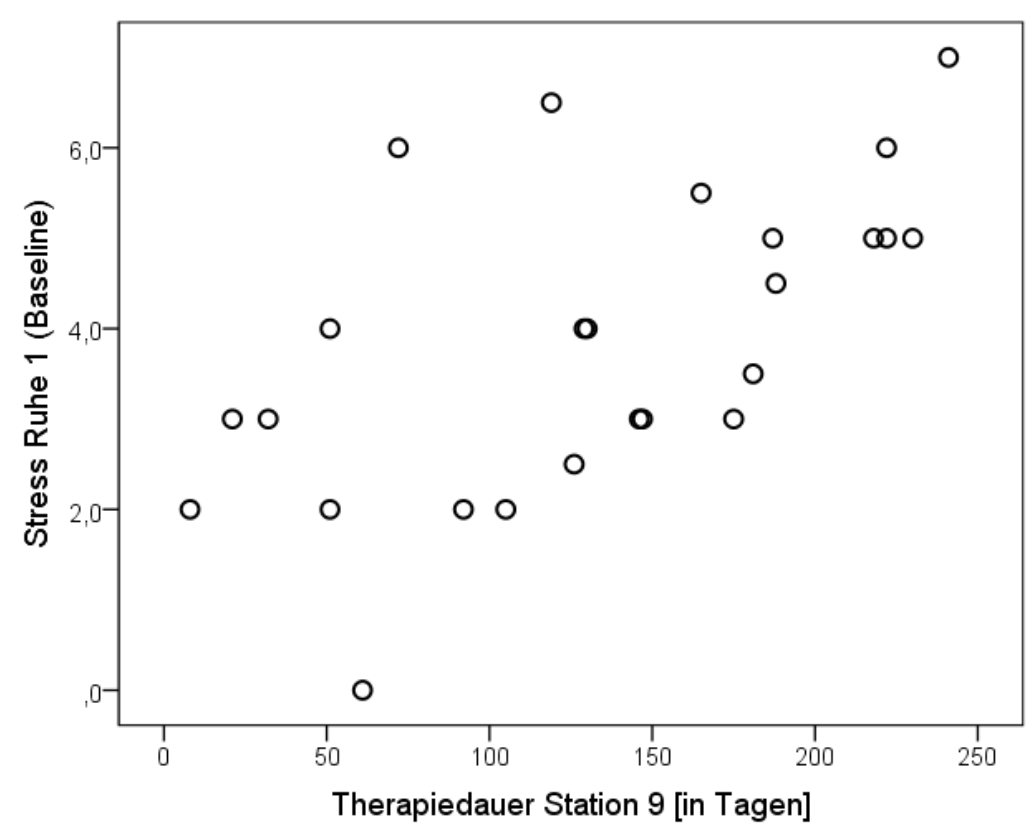

Abbildung 17: Streudiagramm zur graphischen Darstellung der Korrelation zwischen dem Stressempfinden in der Phase Ruhe 1 zum Messzeitpunkt Baseline und der Variable „Therapiedauer Station 9“ (stationäre Therapie auf der Station 9 des Asklepios Fachklinikums Göttingen in Tagen)

\subsection{Die Reagibilität und mögliche Einflussfaktoren}

\subsubsection{Deskriptive Betrachtung der Reagibilität}

Bei der Berechnung der $\Delta \mathrm{R}_{\text {Rechnen }}$ - und $\Delta \mathrm{R}_{\text {Babyschreien }}$-Scores zur Änderung der Recovery (s. Kap. 3.5.1) entstehen die Recoveryscores $\left(\mathrm{R}_{\text {Rechnen }}\right.$ bzw. $\left.\mathrm{R}_{\text {Babyschreien }}\right)$, die neben $\Delta \mathrm{R}$ Scores an dieser Stelle genauer betrachtet werden sollen. Sie bilden ein Maß für die Stressreagibilität der Patientinnen. Tabelle 20 fasst die Mittelwerte und Standardabweichungen der $\Delta \mathrm{R}$-Scores sowie der Recoveryscores für die verschiedenen Parameter zusammen. Für die

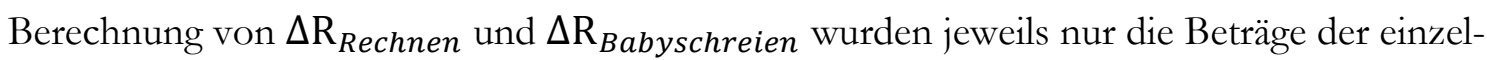
nen Recoveryscores von jeder Patientin verwendet, sodass die $\Delta \mathrm{R}$-Scores nicht aus den hier aufgeführten Mittelwerten der Differenzwerte hergeleitet werden können. 
Tabelle 20: Mittelwerte und Standardabweichungen der Recoveryscores und der $\Delta \mathrm{R}_{\text {Rechnen }}$ und $\Delta \mathrm{R}_{\text {Babyschreien }}$-Scores für HR in [bpm], sBP in [mmHg], TPRI in [dyn*s* $\mathrm{m}^{2} / \mathrm{cm}^{5}$ ], PEP in [ms] und BRS in [ms $/ \mathrm{mmHg}$ ] unter Ausschluss von Patientin E1 (s. Kap. 4.2.5)

\begin{tabular}{|c|c|c|c|c|c|}
\hline & HR & sBP & TPRI & PEP & BRS \\
\hline $\begin{array}{c}\boldsymbol{R}_{\text {Rechnen Baseline }} \\
\text { (Rechnen - Musik Baseline) }\end{array}$ & $\begin{array}{l}M=6,1 \\
S D=3,4 \\
N=23\end{array}$ & $\begin{array}{l}M=4,0 \\
S D=9,2 \\
N=23\end{array}$ & $\begin{array}{l}M=-153,7 \\
S D=246,8 \\
N=23\end{array}$ & $\begin{array}{l}M=-3,3 \\
S D=5,5 \\
N=23\end{array}$ & $\begin{array}{l}M=-2,7 \\
S D=4,0 \\
N=23\end{array}$ \\
\hline $\begin{array}{c}\boldsymbol{R}_{\text {Rechnen Follow up }} \\
\text { (Rechnen - Musik } \\
\text { Follow-up) }\end{array}$ & $\begin{array}{l}M=5,0 \\
S D=3,7 \\
N=25\end{array}$ & $\begin{array}{l}M=3,2 \\
S D=7,3 \\
N=25\end{array}$ & $\begin{array}{l}M=-263,8 \\
S D=347,7 \\
N=24\end{array}$ & $\begin{array}{l}M=-4,8 \\
S D=8,2 \\
N=23\end{array}$ & $\begin{array}{l}M=-2,2 \\
S D=3,8 \\
N=25\end{array}$ \\
\hline $\begin{array}{c}\boldsymbol{R}_{\text {Babyschreien Baseline }} \\
\text { (Babyschreien - Musik } \\
\text { Baseline) } \\
\end{array}$ & $\begin{array}{l}M=1,8 \\
S D=4,4 \\
N=23\end{array}$ & $\begin{array}{l}M=0,02 \\
S D=6,9 \\
N=23\end{array}$ & $\begin{array}{l}M=-63,9 \\
S D=232,9 \\
N=23\end{array}$ & $\begin{array}{l}M=-2,7 \\
S D=6,4 \\
N=23\end{array}$ & $\begin{array}{l}M=-0,8 \\
S D=2,7 \\
N=23\end{array}$ \\
\hline $\begin{array}{c}\boldsymbol{R}_{\text {Babyschreien Follow up }} \\
\text { (Babyschreien - Musik } \\
\text { Follow-up) }\end{array}$ & $\begin{array}{l}M=1,3 \\
S D=3,8 \\
N=25\end{array}$ & $\begin{array}{l}M=-0,4 \\
S D=9,7 \\
N=25\end{array}$ & $\begin{array}{l}M=-45,3 \\
S D=335,1 \\
N=24\end{array}$ & $\begin{array}{l}M=-0,7 \\
S D=4,7 \\
N=23\end{array}$ & $\begin{array}{l}M=-0,9 \\
S D=3,0 \\
N=25\end{array}$ \\
\hline $\begin{array}{c}\Delta \mathbf{R}_{\text {Rechnen }} \\
\left(R_{\text {Rechnen Baseline }}-\right. \\
\left.R_{\text {Rechnen Follow up }}\right)\end{array}$ & $\begin{array}{l}M=1,0 \\
S D=3,1 \\
N=23\end{array}$ & $\begin{array}{l}M=2,0 \\
S D=7,7 \\
N=23\end{array}$ & $\begin{array}{l}M=-80,1 \\
S D=356,6 \\
N=22\end{array}$ & $\begin{array}{l}M=-2,5 \\
S D=7,9 \\
N=21\end{array}$ & $\begin{array}{l}M=-0,4 \\
S D=4,4 \\
N=23\end{array}$ \\
\hline $\begin{array}{c}\Delta \mathbf{R}_{\text {Babyschreien }} \\
\left(R_{\text {Babyschreien Baseline }}-\right. \\
R_{\text {Babyschreien Follow up })} \\
\end{array}$ & $\begin{array}{l}M=0,9 \\
S D=2,8 \\
N=23\end{array}$ & $\begin{array}{l}M=-2,4 \\
S D=7,2 \\
N=23\end{array}$ & $\begin{array}{l}M=-55,7 \\
S D=211,8 \\
N=22\end{array}$ & $\begin{array}{l}M=1,0 \\
S D=7,0 \\
N=21\end{array}$ & $\begin{array}{l}M=-0,4 \\
S D=3,1 \\
N=23\end{array}$ \\
\hline
\end{tabular}

Die Hypothese 3b erwartet eine höhere Reagibilität im Follow-up. Ein Blick auf die Recovery- und $\Delta \mathrm{R}$-Scores zeigt, dass sich Baseline und Follow-up nur sehr geringfügig bezüglich der Recovery bzw. der Reagibilität unterscheiden. Einzig für den TPRI ergeben sich deutlichere Unterschiede zugunsten des Follow-ups, diese sind jedoch in der graphischen Auswertung nicht auszumachen (s. Kap. 4.3.3). Insgesamt ist somit kein klarer Trend in der deskriptiven Analyse erkennbar, sodass die Hypothese $3 \mathrm{~b}$ nicht angenommen werden kann.

\subsubsection{Mögliche Einflussfaktoren}

Für die beiden $\Delta \mathrm{R}$-Scores zur Änderung der Recovery der verschiedenen Parameter wurden jeweils Korrelationen mit folgenden Variablen gerechnet:

- mit den Differenzen (Baseline - Follow-up) des Stressempfindens in Ruhe 1 und Musik (Hypothese 4)

- mit den Differenzen (Baseline - Follow-up) der Gesamtwerte der psychometrischen Fragebögen (GSI der SCL-90-R, BDI-Summenwert, IES-R Übererregung, IES-R Intrusion, IES-R Vermeidung, BPI-Cut-20, BSL-Gesamtskala sowie FDS-Gesamtskala) (Hypothese 5) 
- mit „Therapiedauer gesamt“ (gesamte stationäre und teilstationäre psychiatrische Behandlung inklusive Station 9 und Tagesklinik zwischen Baseline und Follow-up, in Tagen) sowie mit „Therapiedauer Station 9“ (die ausschließlich auf der Station 9 des Asklepios Fachklinikums Göttingen stattgefundene Therapie zwischen Baseline und Follow-up, in Tagen) (Hypothese 6)

Zudem wurde die Korrelation für $\Delta \mathrm{R}_{\text {Rechnen }}$ und die Differenz (Baseline - Follow-up) des Stressempfindens in Rechnen und für $\Delta \mathrm{R}_{\text {Babyschreien }}$ und die Differenz des Stressempfindens in Babyschreien ermittelt.

Tabelle 21: Alle einseitig signifikanten Korrelationen der Scores $\Delta \mathrm{R}_{\text {Rechnen }}$ und $\Delta \mathrm{R}_{\text {Babyschreien }}$ der Parameter HR, sBP, TPRI, PEP und BRS mit den oben genannten Variablen. Die Patientin E1 wurde für diese Berechnungen ausgeschlossen (s. Kap. 4.2.5)

\begin{tabular}{|c|c|c|c|c|c|}
\hline & \multicolumn{5}{|c|}{$\Delta \mathbf{R}_{\text {Rechnen }}$ (Änderung Recovery) } \\
\hline & $\Delta \mathrm{R}_{H R R e}$ & $\triangle \mathrm{R}_{S B P R e}$ & $\Delta \mathrm{R}_{\text {TPRI Re }}$ & $\triangle \mathrm{R}_{P E P R e}$ & $\Delta \mathrm{R}_{B R S R e}$ \\
\hline $\begin{array}{c}\text { Scl-90-R GSI } \\
\text { (Differenz: Baseline - Follow-up) }\end{array}$ & & & $\begin{array}{l}r=-0,44 \\
p<0,05\end{array}$ & & \\
\hline $\begin{array}{c}\text { BDI } \\
\text { (Differenz: Baseline - Follow-up) }\end{array}$ & $\begin{array}{l}r=-0,54 \\
p<0,01\end{array}$ & & & & \\
\hline $\begin{array}{c}\text { BPI-Cut-20 } \\
\text { (Differenz: Baseline - Follow-up) }\end{array}$ & & & $\begin{array}{l}r=-0,61 \\
p<0,01\end{array}$ & & \\
\hline $\begin{array}{c}\text { BSL-Gesamtskala } \\
\text { (Differenz: Baseline - Follow-up) }\end{array}$ & & & $\begin{array}{l}r=-0,39 \\
p<0,05\end{array}$ & & \\
\hline \multirow[t]{3}{*}{ „Therapiedauer gesamt“ } & & & & $\begin{array}{l}r=-0,37 \\
p<0,05\end{array}$ & \\
\hline & \multicolumn{5}{|c|}{$\Delta \mathbf{R}_{\text {Babyschreien }}$ (Änderung Recovery) } \\
\hline & $\Delta \mathrm{R}_{H R B}$ & $\triangle \mathrm{R}_{S B P B}$ & $\Delta \mathrm{R}_{T P R I B}$ & $\triangle \mathrm{R}_{P E P B}$ & $\Delta \mathrm{R}_{B R S B}$ \\
\hline „Therapiedauer gesamt“ & & & & & $\begin{array}{l}r=-0,38 \\
p<0,05\end{array}$ \\
\hline
\end{tabular}

Tabelle 21 listet alle signifikanten Korrelationen aus diesen Berechnungen auf. Entsprechend der 4., 5. und 6. Hypothese wurde einseitig getestet. Demnach finden sich in Tabelle 21 nur negative Korrelationen. Je negativer der $\Delta \mathrm{R}$-Score desto höher ist die Reagibilität im Followup im Vergleich zum Zeitpunkt Baseline. Dagegen bedeuten besonders positive Werte bei den anderen Variablen eine besonders lange Therapiedauer, ein besonders niedriges Stressempfinden im Follow-up gegenüber der Baseline-Messung oder aber besonders niedrige (und damit weniger pathologische) Werte in den psychometrischen Fragenbögen im Followup gegenüber der Baseline-Messung. Hieraus ergibt sich eine den Hypothesen entsprechende Korrelation nur, wenn diese negativ ist. Eine Bonferroni-Korrektur des alpha-Niveaus wurde nicht vorgenommen. 
Insgesamt sind gemessen an der Zahl der berechneten Korrelationen nur wenige signifikante Ergebnisse zu beschreiben. Oft korreliert der TPRI, für den sich jedoch bei der deskriptiven Betrachtung der $\Delta \mathrm{R}$-Scores ein uneindeutiges Verhalten offenbarte, wenn man die graphische Analyse der TPRI-Mittelwerte miteinbezieht (s. Kap. 4.5.1). Eine hochsignifikante Korrelation ist zwischen der Differenz des BDI (Baseline - Follow-up) und dem $\Delta R_{H R}$ Rechnen zu beschreiben (s. Abb. 18). Zudem finden sich signifikante Korrelationen zwischen der „Therapie gesamt“" und $\Delta R_{P E P \text { Rechnen }}$ bzw. $\Delta R_{B R S \text { Babyschreien. }}$

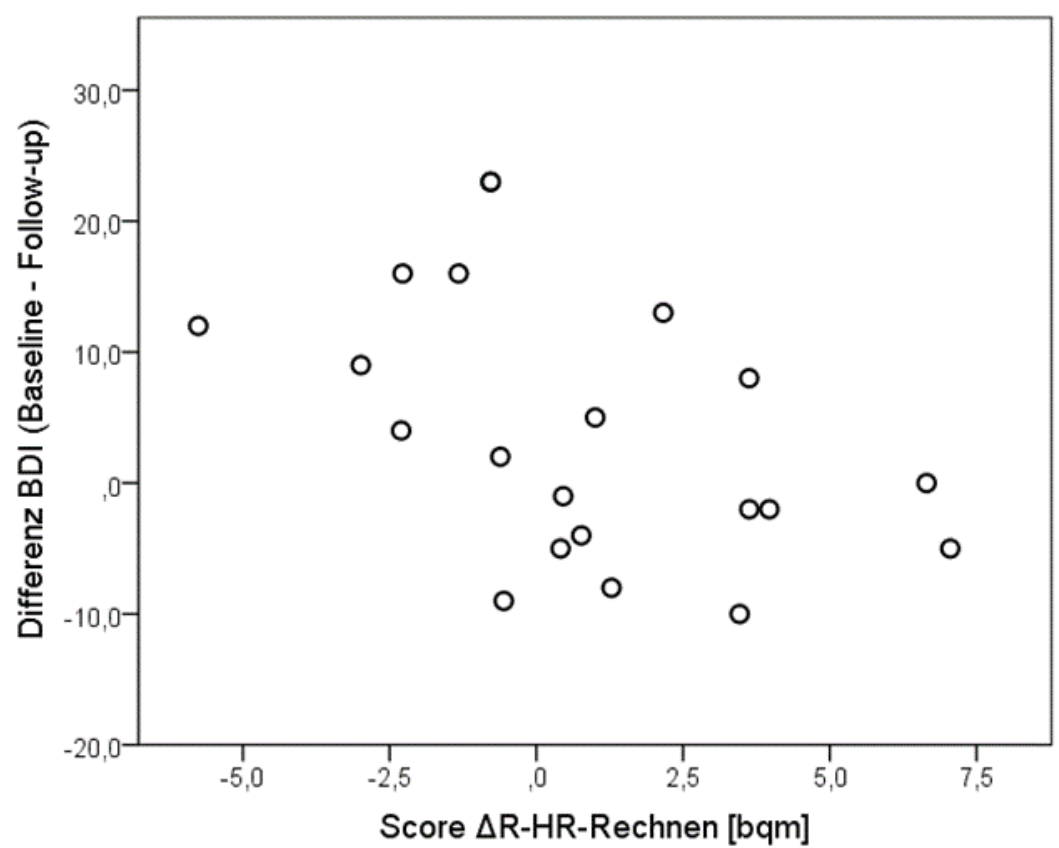

Abbildung 18: Streudiagramm zur graphischen Darstellung der Korrelation zwischen der Differenz des BDI (Baseline - Follow-up) und dem Score $\Delta R_{H R \text { Rechnen }}$ in [bpm]. Die Patientin E1 ist hierbei ausgeschlossen (s. Kap. 4.2.5) 


\section{Diskussion}

\subsection{Hypothese 1: Das Stressempfinden}

Das subjektive Stressempfinden ist ein wichtiger Erfolgsparameter, der auch für die Patientinnen greifbar ist. Gleichzeitig kann anhand des Stressempfindens die Wirksamkeit der Stressoren evaluiert werden: Beide in der vorliegenden Untersuchung eingesetzten Stressoren lösten eine deutliche und etwa gleich starke subjektive Stressreaktion aus. Dass sich kein signifikanter Unterschied zwischen Ruhe 1 und Entspannungsmusik zeigte, spricht wiederum für eine suffiziente subjektive Recovery.

In der Baseline-Messung konnte Bornschein durchgängig signifikant höhere Stresswerte für die PTBS-Patientinnen im Vergleich zur Kontrollgruppe nachweisen (Bornschein 2014). Die Hypothese 1 erwartet ein gegenüber der Baseline-Messung gesunkenes Stressempfinden im Follow-up. Dies konnte bestätigt werden: Über alle Phasen hinweg war das Stressempfinden im Follow-up geringer. Dieser Unterschied war - mit Ausnahme von Ruhe 1 - auch signifikant. Die Patientinnen empfanden den Stress durch den Stresstest im Follow-up somit als geringer. Dies kann als Therapieerfolg interpretiert werden. Durch die Therapie und antrainierte Stressbewältigungsstrategien wurde der Stresstest im Follow-up besser bewältigt. Gleichzeitig muss jedoch auch ein möglicher Wiederholungseffekt bei der Interpretation beachtet werden.

Für einen Vergleich mit ähnlich aufgebauten Studien erscheint diejenige von Lomb et al. besonders geeignet (Lomb et al. 2015, Lomb 2011). Ebenfalls unter Einsatz des Task Force ${ }^{\circledR}$ Monitors untersuchten sie bei 77 Patienten, ob sich eine vier- bis sechswöchige stationäre Psychotherapie im Rahmen einer akutpsychosomatischen Behandlung auf die kardiovaskuläre Regulation auswirkt. Hauptdiagnose war bei etwas mehr als einem Drittel der Patienten eine depressive Störung, bei einem Drittel eine somatoforme Störung und bei dem restlichen Drittel anderweitige Störungsbilder. Neben einer Vielzahl an kardiovaskulären Parametern wurde auch das subjektiven Stressempfinden bei Aufnahme und Entlassung jeweils während eines Stresstests erfasst. Wie in der vorliegenden Arbeit wurde eine von 0 bis 10 reichende numerische Analogskala verwendet. Der Stresstest beinhaltete neben Ruhephasen zwei Phasen der Taktatmung sowie einen Rechen- und einen anger-recall-Test. Auch Lomb berichtet ein im Vergleich zum ersten Messzeitpunkt durchschnittlich geringeres Stressempfinden der Patienten im Follow-up. Diese Beobachtung gilt für das Stressempfinden von allen vier betrachteten Phasen, wobei die anfängliche Ruhephase wiederum den geringsten Abstand zwischen den beiden Messzeitpunkten aufweist. Das Rechnen verzeichnete dagegen die größte Veränderung (Lomb 2011). Interessanterweise befindet sich das Ausmaß der Verringerung im Stressempfinden für beide Studien in einem ähnlichen Rahmen. Bei beiden Studien fand zwischen den beiden Messungen eine Therapie statt, die die Verbesserungen im Stressempfinden bewirkt haben könnte. Bei beiden kommt jedoch auch der Wiederholungseffekt als Erklärung in Frage. Hierbei hätte man aber deutlichere Ergebnisse bei Lomb 
erwartet - schließlich fanden bei ihr die beiden Stressuntersuchungen innerhalb von sechs Wochen statt, während in der vorliegenden Studie eine Zeitspanne von etwa drei Jahren zwischen den Messungen lag. Je dichter zwei Messungen aufeinander folgen, desto größer ist der Wiederholungseffekt einzuschätzen. Geht man davon aus, dass der Wiederholungseffekt bei den PTBS-Patientinnen durch die deutlich längere Zeitspanne zwischen den beiden Messungen niedriger als bei Lombs Kohorte ausfallen müsste, stellt sich die Frage, warum das Stressempfinden in beiden Studien ähnlich stark gesunken ist. Eine mögliche Erklärung ist eine stärkere Therapiewirkung bei den PTBS-Patientinnen.

Zusammenfassend kann festgehalten werden, dass der während der Untersuchung empfundene Stress im Follow-up geringer war. Ein Wiederholungseffekt ist bei einem Abstand von drei Jahren zwischen den Messungen als niedrig einzuschätzen, v. a. im Vergleich zu der Studie von Lomb (2011). Das verringerte Stressempfinden weist auf möglicherweise eingetretene Therapieeffekte hin.

\subsection{Hypothese 2: Die Psychometrie}

Die Hypothese 2 besagt, dass zum Zeitpunkt Follow-up das psychische Befinden der Patientinnen besser war als bei der Baseline-Untersuchung. Auch dies könnte ein Indiz für einen Therapieerfolg darstellen. Die SCL-90-R erlaubt eine erste Einschätzung über die allgemeine psychische Belastung der Kohorte. Hier ergaben sich im Mittel für alle Subskalen und Globalwerte pathologisch hohe Werte. Von Baseline zu Follow-up sank der globale Kennwert GSI, allerdings nicht signifikant. Die beiden Subskalen Aggressivität/Feindseligkeit sowie paranoides Denken im Follow-up waren sogar höher als bei der Baseline-Messung. Alle anderen Skalenwerte waren im Follow-up niedriger, die Subskalen Ängstlichkeit und Unsicherheit im Sozialkontakt auch signifikant. Das BDI zeigt die stärkste Veränderung bei den psychometrischen Fragebögen, im Follow-up erreichten die Teilnehmerinnen signifikant weniger pathologische Ergebnisse als noch in der Baseline-Messung. Damit hat sich die depressive Symptomlast als der dynamischste psychometrische Faktor erwiesen. Mit der IESR werden die PTBS-typischen Symptomkomplexe Intrusion, Übererregung und Vermeidung evaluiert, wobei sich insbesondere die Skala Vermeidung zum Follow-up hin (signifikant) besserte. Die dissoziative Symptomatik, gemessen mit dem FDS, veränderte sich dagegen kaum zwischen Follow-up und Baseline. Das BPI bezieht sich auf Merkmale der BPS und zeigte ein durchmischtes Bild mit einem gegensätzlichen Verhalten der verschiedenen Skalen. Auch wenn der BPI-Cut-20 als globaler Kennwert gegenüber der Baseline-Untersuchung leicht gesunken ist, muss insgesamt das BPI als unverändert bilanziert werden. Die BSL bildet die aktuelle Symptomlast bei Borderline-Patienten ab. Tendenziell scheint die Symptomlast hier sogar etwas angestiegen zu sein.

Für eine Einordnung dieser Ergebnisse lohnt ein Blick auf Studien, die eine Bewertung der angewandten Therapieverfahren anhand der gleichen psychometrischen Fragebögen vornahmen. Wichtige Bestandteile der von Sachsse entwickelten Therapie auf der Station 9 des 
Asklepios Fachklinikums Göttingen (Herbold und Sachsse 2008) sind die Psychodynamisch Imaginative Traumatherapie (PITT; Reddemann 2011), das Eye Movement Desensitization and Reprocessing (EMDR; Shapiro 1999) sowie die Dialektisch-Behaviorale Therapie (DBT; Linehan 1993). Sachsse et al. (2006) evaluierten dieses spezifische Behandlungskonzept für komplexe Traumafolgestörungen (s. Kap. 1.5.1) und berichteten übereinstimmend mit der vorliegenden Arbeit von signifikanten Besserungen der Skalen Primitive Abwehr und Objektbezogenheit (BPI) und Vermeidung (IES). Ein Widerspruch ist jedoch, dass eine signifikante Besserung dissoziativer Symptome, aber keine signifikante Verringerung des BDI nachgewiesen wurde. Die Ergebnisse waren auch nach einem Jahr stabil (Sachsse et al. 2006).

Eine Therapieevaluation einer sechswöchigen stationären PIT'T zeigte, dass Patientinnen mit einer chronischen Traumatisierung in der Kindheit direkt im Anschluss der Therapie u. a. signifikante Verbesserungen bei den Fragebögen SCL-90-R, BDI und IES verzeichneten. Die mithilfe des FDS gemessene dissoziative Symptomatik verringerte sich dagegen nicht. In einem Follow-up nach sechs Monaten konnten die positiven Effekte bei der SCL-90-R und dem BDI weiterhin beobachtet werden. Bei der IES zeigten sich zwar auch hier niedrigere Werte im Vergleich zu dem Erhebungszeitpunkt vor der Therapie, diese waren nach sechs Monaten jedoch nicht mehr signifikant (Lampe et al. 2008). In einer ähnlichen Studie konnte auch nach zwei Jahren eine signifikante Verbesserung der depressiven Symptomatik anhand des BDI nachgewiesen werden. Für die dissoziative Symptomatik fand sich wiederum keine signifikante Veränderung durch die Therapie (Lampe et al. 2014). Damit entsprechen die Resultate der beiden Studien bezüglich BDI, IES und FDS der hier vorliegenden Arbeit und unterstützen im Gegensatz zu Sachsse et al. (2006) die Beobachtung, dass die dissoziative Symptomatik wenig durch die Therapie beeinflussbar zu sein scheint, während die depressive Symptomatik auch nach längerer Zeit besonders von der Therapie profitiert. Dass die Verringerung der SCL-90-R im Gegensatz zu Lampe et al (2008) in dieser Studie keine Signifikanz erreichte, könnte der kleineren Stichprobe und dem größeren zeitlichen Abstand geschuldet sein. Eine ähnliche Tendenz ist zwar erkennbar, bleibt aber aufgrund der fehlenden Signifikanz spekulativ.

Auch dem EMDR-Therapieverfahren wurde vielfach die Therapiewirkung bescheinigt (Shapiro 2014, Chen et al. 2015). In einer Untersuchung von größtenteils weiblichen Flüchtlingen mit pathologischen IES-R-Werten konnten sowohl für die IES-R als auch für das BDI II signifikante Verringerungen nach einer EMDR-Therapie nachgewiesen werden (Acarturk et al. 2015). Ein Beispiel dafür, dass die EMDR-Therapie sowohl auf die klassische PTBSSymptomatik als auch auf die mit der PTBS einhergehenden depressiven Symptome Wirkung zeigt. Die EMDR-Therapie könnte somit auch Anteil bei der signifikanten Verbesserung des BDI in dieser Studie haben. Gleichzeitig ist jedoch auch von der Studie von Macklin et al. (2000) zu berichten, die ein 5-Jahres-Follow-up nach einer EMDR-Therapie bei 13 Kriegsveteranen mit PTBS durchführten. Die Effekte, die sich direkt im Anschluss an die Therapie präsentierten, waren nach fünf Jahren nicht mehr festzustellen. Tatsächlich zeigte sich sogar parallel zu einer Kontrollgruppe ohne EMDR-Therapie eine leichte Verschlechterung, die 
sich beispielsweise am signifikanten Ansteigen der SCL-90-R festmachen ließ (Macklin et al. 2000). Zu der Therapiewirkung bei der PTBS gibt es nur wenige Studien mit einer so langen Spanne zwischen Therapie und Follow-up wie es in der vorliegenden Studie oder bei Macklin et al. der Fall ist. Die Ergebnisse von Macklin et al. (2000) legen jedoch nahe, dass viele Effekte, die in anderen Studien direkt nach der Therapie oder bei einem früheren Follow-up beschrieben werden, bei einem 3-Jahres-Follow-up wie in der vorliegenden Studie eventuell nicht mehr gemessen werden können.

Die Einbeziehung der DBT in das Behandlungskonzept ergibt sich aus der Abgrenzung der komplexen Traumafolgestörungen mit der häufig komorbiden BPS von der „klassischen“ PTBS. Während Sachsse PITT, EMDR und DBT zu einer Therapie verbindet, erschienen in den letzten Jahren auch Studien, die eine Kombination aus traumafokussierter kognitiver Verhaltenstherapie und DBT erprobten. Bei Teilnehmerinnen mit einer PTBS nach sexuellem Missbrauch in der Kindheit konnte eine Abnahme der PTBS-Symptomatik sowie eine signifikante Reduktion von BDI und SCL-90-R sechs Wochen nach der Therapie festgestellt werden (Steil et al. 2011). Bei einer ganz ähnlichen Kohorte erfolgte ein Vergleich der Kombination „traumafokussierte kognitive Verhaltenstherapie und DBT“ mit den bislang üblichen Therapiekonzepten, wobei sich eine signifikant stärkere Reduktion der PTBSSymptomatik bei dem Therapieregime mit DBT ergab. Dabei war die Therapiewirkung durch die DBT nicht an das Vorhandensein einer komorbiden BPS gebunden (Bohus et al. 2013). Die DBT ist somit auch unabhängig von der Kombination mit EMDR und PITT eine erfolgreiche Behandlungsmethode der komplexen Traumafolgestörungen.

Alle drei Hauptkomponenten der Therapie auf der Station 9 des Asklepios Fachklinikums Göttingen können somit zu einer Besserung des psychometrischen Befunds und damit zu dem Wohlbefinden der Patientinnen beigetragen haben. Die Resultate der vorliegenden Studie stimmen zudem mit den Beobachtungen vorausgegangener Studien weitgehend überein. Skalenwerte, die sich bereits in der Vergangenheit als zuverlässige Kennzeichen einer erfolgreichen Therapie erwiesen haben, verzeichneten auch in dieser Studie Verbesserungen. Gleichzeitig präsentierten sich jene Symptomkomplexe, die sich in dieser Studie kaum von der Therapie beeinflusst zeigten, auch in vorausgegangenen Studien mit einer ähnlich geringfügigen Veränderung.

Bei der Interpretation der Ergebnisse sollte außerdem eine Differenzierung in Symptomskalen und Persönlichkeitsskalen Beachtung finden. Das BPI bildet eine typische Persönlichkeitsskala, indem es die Borderline-Persönlichkeitsstörung evaluiert. Persönlichkeitsmerkmale und -störungen sind ihrer Definition entsprechend intraindividuell sehr konstante Faktoren. So überrascht es wenig, dass das BPI mit Ausnahme der Skala Primitive Abwehr und Objektbezogenheit keine signifikante Verbesserung zwischen Baseline und Follow-up verzeichnete. Die BSL dagegen bildet die Symptome der BPS ab und sollte damit änderungssensitiver sein. Aber auch hier weist die Follow-up-Messung keine Besserung auf. Zwar ist die DBT eine Komponente bei der Therapie auf der Station 9 des Asklepios Fachklinikums 
Göttingen. Im Fokus steht jedoch die traumafokussierte Behandlung, während die DBT insbesondere zur Stabilisierung dient (Müller und Sachsse 2010). Die BPS gilt selbst bei umfangreicher DBT-Behandlung als schwierig therapierbar, sodass eine primäre PTBSBehandlung vielleicht nicht ausreicht, um gleichzeitig die BPS-Symptomlast zu verringern. Zudem ist es denkbar, dass die BSL direkt im Anschluss an die Behandlung eine Besserung anzeigte, die jedoch nach drei Jahren nicht mehr abzubilden ist. Für den FDS war ebenfalls eine stärkere Veränderung erwartet worden. Auch bei Lampe et al. (2008, 2014) blieb der FDS trotz Therapie und der Besserung einiger anderer Parameter weitgehend unverändert, sodass es sich hier um eine Therapierefraktärität der dissoziativen Symptomatik handeln könnte. Weitere Forschung ist an dieser Stelle notwendig, um diese Beobachtung zu bestätigen und um ggf. spezielle Therapiekonzepte für die dissoziative Symptomatik bei den komplexen Traumafolgestörungen zu entwickeln und zu integrieren. SCL-90-R, BDI und IES-R sind klassische Symptomskalen und damit vergleichsweise beeinflussbar. Dies spiegelt sich auch in den Ergebnissen wider, zeigen sich doch Verbesserungen bezüglich der depressiven Komorbidität, aber auch positive (allerdings insignifikante) Tendenzen bezüglich der PTBS und der allgemeinen psychischen Belastung. Dies gilt insbesondere unter der Annahme, dass der große zeitliche Abstand zwischen Therapie und Follow-up viele Effekte deutlich abgeschwächt haben könnte. Dennoch ist gerade der Befund eines späten Follow-ups von Interesse, ist es doch das Ziel jeder Therapie möglichst langfristige Effekte zu bewirken.

\subsection{Hypothese 3: Die Stressphysiologie}

\subsubsection{Ruhebedingungen}

Durch die Hypothese 3a rücken bei der Auswertung die Ruhebedingungen (Ruhe 1) in den Fokus. Für die Baseline-Messung ist graphisch eine von der Kontrollgruppe abweichende Ruheaktivierung der PTBS-Patientinnen erkennbar. Bei HR, sBP und TPRI findet sich eine Tendenz zu höheren Werten, während die BRS eher niedrigere Werte aufweist und die PEP sich nicht von der Kontrollgruppe zu unterscheiden scheint. Für einen ausführlichen Vergleich von Kontrollgruppe und PTBS-Patientinnen in Ruhe inklusive Signifikanztestung sei auf die Dissertation von Bornschein verwiesen. Für Bornschein sind die kardiovaskulären Abweichungen der PTBS-Patientinnen am ehesten mit einer vagalen Hypoaktivität in Ruhe erklärbar. Hinweise auf ein generelles sympathisches Hyperarousal fand sie im Gegensatz zu einigen vorausgegangenen Studien nicht (Bornschein 2014). Die Hypothese 3a erwartet eine Annäherung der Follow-up-Gruppe an die Werte der Kontrollgruppe in Ruhe. Einzig die HR verhielt sich erwartungsgemäß und war im Follow-up signifikant niedriger als in der Baseline-Messung. Gemäß der Sensitivitätsanalyse (s. Anhang, Kap. 8.6) war dieser Effekt jedoch größtenteils durch Änderungen in der Betablockereinnahme konfundiert. Die anderen Parameter veränderten sich dagegen nicht gemäß der Hypothese. Die Werte des TPRI waren gegenüber der Baseline-Messung in der zweiseitigen explorativen Testung sogar 
signifikant erhöht. Der sBP und die BRS wiesen einen weitgehend zur Baseline-Untersuchung unveränderten Befund auf. Eine der Hypothese gegenläufige deskriptive Verkürzung der PEP erreichte explorativ keine Signifikanz.

Auch wenn die Datenlage teilweise inkonsistent ist, zählt die erhöhte Herzrate in Ruhe zu den am häufigsten berichteten Befunden zur Psychophysiologie der PTBS (Pole 2007). Eine Verringerung der HR wäre von besonderem Interesse, könnte sie doch als eines der vermuteten kardiovaskulären Hauptsymptome der PTBS eine grundsätzliche Besserung der Symptomatik kennzeichnen. Die Herzrate wird sowohl sympathisch als auch vagal reguliert. Lange wurde die erhöhte Ruhe-HR einzig einer Hyperaktivität des sympathischen Systems zugeschrieben. In den letzten Jahren mehrten sich jedoch die Hinweise, dass eine Hypoaktivität des Parasympathikus eine entscheidende Rolle spielen könnte (Sack et al. 2004, Hopper et al. 2006, Bornschein 2014). Hiermit stimmt die Erkenntnis überein, dass der vagale Einfluss auf die HR in Ruhe deutlich überwiegt (Hopper et al. 2006). Folglich könnte die im Vergleich zur Baseline-Messung im Follow-up niedrigere HR einem angestiegenden vagalen Tonus entsprechen, aber auch eine verringerte $\beta$-adrenerge Aktivierung bleibt eine mögliche Erklärung. Beides könnte als eine Verbesserung im Sinne einer Annäherung an die Kontrollgruppe interpretiert werden. Gleichzeitig ist jedoch ein medikamentöser Konfundierungseffekt nicht außer Acht zu lassen. Wie in Kapitel 4.1.5 beschrieben, nahmen die Patientinnen eine Vielzahl an Medikamenten ein. Insbesondere die Betablockermedikation hat dabei eine Wirkung auf die Herzrate. Um einem möglichen Einfluss durch Betablocker auf den Grund zu gehen, wurden die durchgeführten Berechnungen durch eine Sensitivitätsanalyse ergänzt (s. Anhang, Kap. 8.6). Bei zwei Patientinnen waren zwischen Baseline und Follow-up Betablocker neu verordnet worden. Eine dieser beiden Patientinnen (E1) war bei den $\Delta$ R-Scores als deutlicher Extremwert aufgefallen (s. Kap. 4.2.5), woraufhin sie für die $\Delta$ R-Scores sowie alle Rechnungen mit den Stressphasen ausgeschlossen wurde. Einer dritten Patientin wurde die Betablockermedikation zwischen den beiden Messzeitpunkten abgesetzt. In der Sensitivitätsanalyse erfolgten nun die Rechnungen aus den Kapiteln 4.3.4 bis 4.3.6 unter Ausschluss dieser drei Patientinnen, wobei zu beachten ist, dass in den Kapiteln 4.3.5 und 4.3.6 die Patientin E1 bereits regulär ausgeschlossen ist. Die Analyse konzentriert sich dabei auf die Effektstärke. Hiernach ist ein Betablockereffekt insbesondere bei der Ruhe-HR zu verorten. Während der Abfall von im Mittel 77,8 bpm in Baseline auf 74,2 bpm im Follow-up noch eine signifikante Effektstärke von $d=0,37$ aufweist, erreicht die Effektstärke nach Ausschluss der drei Patientinnen nur noch etwa die Hälfte des Wertes $(d=0,19)$. Zwar sinkt auch nach Ausschluss der drei Patientinnen die HR von 76,4 bpm in Baseline auf 74,6 bpm im Follow-up, dieser nun deutlich kleinere Effekt ist jedoch nicht mehr signifikant. Angesichts der verringerten Effektstärke kann somit davon ausgegangen werden, dass ein wesentlicher Anteil des HR-Abfalls zwischen Baseline und Follow-up in Ruhe durch Veränderungen in der Betablockermedikation bedingt sein könnte. Darüber hinaus könnte es aufgrund der Abnahme der Depressivität (signifikant gesunkener BDI) zu einer Reduktion der anticholinergen Medikation wie Trizyklika oder Promethazin zwischen den beiden 
Messzeitpunkt gekommen sein. Auch dieser Mechanismus könnte somit bei der Verringerung der Ruhe-HR im Follow-up einen Anteil gehabt haben. Ein Therapieeffekt im Sinne einer vagalen Steigerung oder einer $\beta$-adrenergen Aktivitätsreduktion kann jedoch nicht bilanziert werden.

Ähnlich starke Verschiebungen durch die Betablockermedikation konnten für die anderen untersuchten Parameter nicht ausgemacht werden. Der sBP zeigte bei beiden Analysen eine sehr geringe Effektstärke, die Effektstärke des TPRI sank um $8 \%$ durch den Ausschluss der drei Patientinnen, während die Effektstärke der PEP um $11 \%$ stieg. Allgemein ist die Effektstärke bei der PEP als mittelgroß einzuschätzen. Für die BRS ergab sich dagegen nur eine kleine Effektstärke, die zwar durch den Ausschluss um $50 \%$ anstieg, aber weiterhin als gering einzuordnen ist. Insgesamt nahmen sieben der Patientinnen zum Zeitpunkt Follow-up Betablocker ein. Es kann nicht ausgeschlossen werden, dass es bei den restlichen fünf Patientinnen, die an beiden Messzeitpunkten Betablocker in der Medikation aufwiesen, zusätzlich zu Dosisänderungen kam. Auf einen generellen Ausschluss aller Patientinnen mit Betablockermedikation wurde jedoch zugunsten der Repräsentativität verzichtet. Betablocker sind eine häufige Medikation bei der PTBS, nicht zuletzt aufgrund der Hyperarousal-Symptomatik und der autonomen Dysfunktion prädisponiert dieses Krankheitsbild für eine Betablockereinnahme. Bornschein (2014) hatte bereits den globalen Einfluss von herzwirksamen Medikamenten auf die Baseline-Messung analysiert, wobei eine isolierte Betrachtung der Betablockermedikation aufgrund zu kleiner Subgruppen nicht möglich war. Die herzwirksame Medikation hatte dabei zu keiner grundlegenden Veränderung der Reaktionsmuster geführt, sondern ging lediglich mit einem über die ganze Messung konstanten Shift einiger Messwerte einher. Gerade die Herzrate zeigte sich jedoch kaum beeinflusst und tendierte sogar zu höheren Werten unter der herzwirksamen Medikation. Eine Ursache hierfür könnte in der antagonistischen Wirkung von Betablocker- und anticholinerger Medikation liegen (beide Medikamentengruppen zählten zur herzwirksamen Medikation), sodass ein Effekt der Betablockermedikation durch die anticholinerge Medikation im Mittel möglicherweise aufgehoben wurde (Bornschein 2014). Nach den gewonnenen Erkenntnissen aus der Sensitivitätsanalyse dieser Studie scheint der Einfluss der Betablockermedikation auf die HR in Ruhe jedoch erheblich, sodass dies in zukünftiger Forschung Berücksichtigung finden sollte. Insbesondere da es sich bei der HR um den am meisten untersuchten Parameter bei der PTBS handelt und bisweilen inkonsistente Ergebnisse berichtet wurden.

Die BRS als vagales Maß zeigte im Follow-up keine signifikante Veränderung und wirkt auch graphisch weitgehend unverändert. Damit deutet der Verlauf der BRS nicht auf eine vagale Aktivitätssteigerung im Sinne eines Therapieeffekts hin. Die von Bornschein (2014) beschriebene vagale Hypoaktivität in Ruhe blieb folglich bestehen. Sie stimmt mit mehreren Studien überein, die ebenfalls einen verringerten Parasympathikus-Tonus in Ruhe bei der PTBS festgestellt hatten (Cohen et al. 1998, Cohen et al. 2000, Hughes et al. 2006, Blechert et al. 2007, Hughes et al. 2007, Hauschildt et al. 2011, Chang et al. 2013). Die PEP gilt als rein $\beta$ adrenerg modulierter Parameter und wird im Gegensatz zu der HR nicht vagal beeinflusst 
(Newlin und Levenson 1979). Die PEP war im Follow-up insignifikant verkürzt. Eine verkürzte PEP gilt allgemein als Zeichen für eine gesteigerte $\beta$-adrenerge Aktivierung und würde somit auch bei Signifikanz nicht den Erwartungen an einen günstigen Therapieeffekt entsprechen. Auch der Ruhe-Blutdruck blieb von Baseline zu Follow-up konstant. Der TPRI war dagegen im Follow-up signifikant höher als in der Baseline-Untersuchung. Das unterschiedliche Verhalten des TPRI im Vergleich zum systolischen Blutdruck mag verwirren, tatsächlich korrelieren (systolischer) Blutdruck und TPRI aber in der Regel nicht miteinander. Dies ist mit dem Einfluss des HZV zu erklären (Joyner et al. 2016). Der TPRI des Followups ist nicht nur in der Ruhe 1-Phase signifikant höher als zum Zeitpunkt der BaselineMessung, sondern während der gesamten Messung. Daher soll eine genauere Diskussion dieses Effekts in Kapitel 5.3.4 erfolgen. Insgesamt ist für das Follow-up eine im Wesentlichen unveränderte kardiovaskuläre Aktivierung in Ruhe zu bilanzieren.

\subsubsection{Reactivity}

Mit der Reactivity soll die autonome Aktivierung durch die Stressoren ausgewertet werden. Dies gibt nicht nur Rückschlüsse auf die Stressreaktion und -reagibilität der Patientinnen zum Messzeitpunkt und im Verlauf, sondern ermöglicht auch eine Evaluation der Stressoren.

\subsubsection{Rechnen}

Für den kognitiven Stressor Rechnen gibt einen breiten Erfahrungshintergrund. Doch nur wenige Studien untersuchten anhand von Rechenstress die Stressregulation bei der PTBS. Auch ist uns bislang keine weitere Studie bekannt, in der die durch das Rechnen ausgelöste Reactivity zur Evaluation eines Therapieverlaufs bei komplexen Traumafolgestörungen genutzt wurde.

Als autonome Antwort auf den Rechenstress finden sich üblicherweise eine Steigerung des Sympathikus- sowie eine Abnahme des Parasympathikustonus. Dabei steht im sympathischen System die $\beta$-adrenergen Aktivierung im Vordergrund (Ottaviani et al. 2006), aber auch eine $\alpha$-adrenerge Aktivierung ist für das Rechnen beschrieben (Winzer et al. 1999, Ring et al. 2000). Im Follow-up stiegen HR und sBP als Reaktion auf das Rechnen deutlich an, die BRS sank - diese Parameter verhielten sich somit erwartungsgemäß. Entgegen den Erwartungen präsentierte sich dagegen die Reactivity von TPRI und PEP: Sie blieben von Ruhe 1 zum Rechnen weitgehend unverändert. In der ANOVA „Reactivity Rechnen“ (Kap. 4.3.5) wurden die durch den Rechenstress ausgelösten Veränderungen der fünf Parameter unter Einbeziehung beider Messzeitpunkte statistisch betrachtet. Der deskriptiv deutliche Anstieg von HR und sBP entspricht hier einer höchstsignifikanten Veränderung, der Abfall der BRS war mit $\mathrm{p}<0,05$ signifikant. Für PEP und TPRI ergaben sich dagegen keine Signifikanzen. Diese Ergebnisse entsprechen weitgehend den von Albrecht (2013) berichteten Zahlen zur Baseline-Kohorte. Auch sie fand höchstsignifikante Effekte bei HR und sBP, der Abfall der BRS erreichte jedoch kein Signifikanzniveau. Im Folgenden sollen die Parameter einzeln 
bezüglich ihres Verhaltens in der Rechenphase und einer möglichen Veränderung von Baseline zu Follow-up diskutiert werden.

Der höchstsignifikante Anstieg der HR unter Rechenstress kann einer $\beta$-adrenergen Aktivierung oder einem vagalen Abfall entsprechen. Es ist wahrscheinlich, dass beide Mechanismen synergistisch wirken, denn eine Reduktion des Parasympathikustonus führt auch zu einer Enthemmung des Sympathikus. Graphisch ist erkennbar, dass zu den beiden Messzeitpunkten der Anstieg der HR parallel verläuft (s. hierzu Abb. 12, S. 64). Die Unterschiede in den Absolutwerten resultieren aus einer während der ganzen Messung im Mittel niedrigeren HR (s. Kap. 5.3.4). Der HR kann somit keine Veränderung in der Rechnen-Reactivity bescheinigt werden. Auch rechnerisch ergaben sich hierfür in der ANOVA keine Signifikanzen. Damit zeigen die PTBS-Patientinnen weiterhin eine geringere Reactivity als die Kontrollgruppe (Bornschein 2014). Der ebenfalls höchstsignifikante Anstieg des systolischen Blutdrucks unter Rechenstress kann durch eine Steigerung des HZV oder durch einen Anstieg des TPRI im Sinne einer Vasokonstriktion vermittelt sein. Da der TPRI jedoch keine signifikante Veränderung zeigt und sich auch graphisch weitgehend unverändert präsentiert, ist von ersterer der beiden Möglichkeiten auszugehen. Eine Steigerung des HZV kann einer $\beta 1$-adrenerge Aktivierung und/oder einer vagalen Aktivitätsminderung entsprechen und ist übereinstimmend mit der Reaktion der Herzrate. Die Reaktion des sBP auf das Rechnen erscheint bei Baseline und Follow-up graphisch ähnlich. Auch in der Reactivity-ANOVA konnte kein signifikanter Zeiteffekt ermittelt werden. Eine Annäherung des Follow-ups an die Kontrollgruppe ist somit auch für den sBP nicht festzustellen. Hierzu ist jedoch anzumerken, dass Bornschein (2014) keinen signifikanten Unterschied zwischen der sBP-Reactivity der PTBS-Patientinnen und der Kontrollgruppe für das Rechnen nachweisen konnte, auch wenn graphisch ein etwas stärkerer Anstieg bei der Kontrollgruppe erkennbar ist.

Die zwischen Baseline und Follow-up unveränderte Stressreaktion von HR und sBP impliziert aber auch, dass für diese beiden Parameter der Befund einer verminderten Reaktivität von Albrecht und Bornschein reproduziert werden konnte (Albrecht 2013, Bornschein 2014, Meyer et al. 2016). Dabei ist die Datenlage zur Reaktivität bei der PTBS keinesfalls konsistent. Häufig wird eine erhöhte Reactivity als charakteristisch für die PTBS angesehen. Untermauert wird diese Aussage durch die Metaanalyse von Pole, die nach der Zusammenschau einer Vielzahl an Studien verstärkte HR-Reaktionen auf schreck- und traumaassoziierte Reize für PTBS-Probanden beschrieb (Pole 2007). Auch aktuelle Arbeiten stimmen mit dem Befund einer erhöhten Stressreaktivität überein (Pineles et al. 2013, Van ’t Wout et al. 2017). Zudem konnte für Frauen, die aufgrund sexuellen Missbrauchs in der Kindheit an einer PTBS litten, eine erhöhte Reaktivität auf traumaspezifische Skripte im Vergleich zu einer traumaexponierten Kontrollgruppe ohne PTBS nachgewiesen werden (Orr et al. 1998). Dies stellt einen Hinweis darauf dar, dass die berichteten Befunde auch für Frauen mit komplexen Traumafolgestörungen gelten könnten. Die Beobachtung einer verminderten Reactivity auf traumaassoziierten Stress bildet dagegen eher eine Ausnahme (Cohen et al. 1998, Cohen et al. 2000). Wie bereits anklang nutzte ein Großteil dieser Studien traumaassoziierte Stressoren, indem 
beispielsweise anhand eines Skripts ein Wiedererinnern des traumatischen Erlebnisses hervorgerufen wurde. Es ist jedoch naheliegend, dass der traumaassoziierte Stress eine Sonderrolle in der Stressregulation der PTBS einnimmt. Diese These konnten Pineles et al. (2013) mit der Tatsache stützen, dass bei 150 traumaexponierten Probanden aus fünf verschiedenen Studien die erhöhte psychophysiologische Antwort auf traumaassoziierte Stressoren einen signifikanten Prädiktor für eine PTBS darstellte, während die Reaktion auf vergleichbare traumaunabhängige Stressoren kein Prädiktor für das Vorliegen einer PTBS war.

Es erscheint daher für eine Einordnung der vorliegenden Arbeit sinnvoller, nur Studien heranzuziehen, die ebenfalls den kognitiven Stressor Rechnen oder andere traumaunabhängige Stressoren bei der PTBS einsetzten. Nur bei wenigen Studien war dies jedoch der Fall. Blanchard et al. (1996) zeichneten bei 105 Überlebenden eines Unfalls mit einem Kraftfahrzeug sowie bei 54 Probanden ohne Verkehrsunfall in der Vorgeschichte u. a. die kardiovaskuläre Antwort auf einen Rechentest in Form von HR und sBP auf. Sie konnten keine Unterschiede in der HR-Antwort zwischen den Gruppen (PTBS, subsyndromale PTBS, keine PTBS, Kontrollgruppe ohne Verkehrsunfall) finden, für den sBP ergab sich aber ein globaler signifikanter Gruppenunterschied. Dabei war die Reactivity des sBP bei den PTBSProbanden am niedrigsten und in der Kontrollgruppe am höchsten. Ein paarweiser Vergleich der Gruppen ergab jedoch keine signifikanten Effekte (Blanchard et al. 1996). Keane et al. (1998) führten ebenfalls einen Rechenstresstest durch, und auch sie berichteten von einer verringerten Stressreaktion von Kriegsveteranen mit aktueller PTBS-Symptomatik im Vergleich zu einer Kontrollgruppe ohne oder mit chronischer PTBS. Auch wenn die Reactivity des sBP ebenfalls niedriger erschien, konnten nur für die HR und den dBP signifikante Unterschiede nachgewiesen werden (Keane et al. 1998). Dafür zeichneten sich Kriegsveteranen mit einem chronischen Erschöpfungssyndrom und einer PTBS durch einen im Vergleich $\mathrm{zu}$ gesunden Kriegsveteranen verminderten sBP-Anstieg als Antwort auf einen Rechentest aus (Peckerman et al. 2003). In einer Dissertation wurden zudem eine PTBS-Gruppe, eine Depressionsgruppe sowie eine gesunde Kontrollgruppe u. a. bezüglich ihrer Reaktivität auf Rechenstress verglichen. Hierbei ergaben sich keine signifikanten Gruppenunterschiede für die Reactivityscores. Rein deskriptiv war jedoch im Mittel die sBP- und HR-Reaktivität der Gruppen PTBS und Depression geringer als die der Kontrollgruppe (Malcolm 2015). Dank der Studie von McDonagh-Coyle et al. (2001) ist ein direkter Vergleich eines traumabezogenen Stressors und eines arithmetischen Tests möglich. Bei 37 Frauen, die sexuellen Missbrauch in der Kindheit erlebt hatten, korrelierte die Reaktivität auf den traumabezogenen Stressor positiv mit der PTBS-Symptomschwere. Die Reaktivität auf den Rechentest korrelierte dagegen negativ mit der Schwere der PTBS-Symptomatik (McDonagh-Coyle et al. 2001).

Die Datenlage für den kognitiven Stressor Rechnen erscheint somit durchaus konsistent und die Hyporeagibilität der PTBS-Patientinnen der vorliegenden Studie steht im Einklang mit dieser. Vieles deutet darauf hin, dass die Reaktivität der PTBS stressorabhängig sein könnte. Es ist denkbar, dass in der Stressphysiologie der PTBS grundsätzlich eine Hyporeaktivität besteht, sodass es bei einem kognitiven Stressor wie dem Rechnen zu einer verminderten 
Reagibilität kommt. Traumanahe Stressoren könnten jedoch eine Ausnahme darstellen, indem sie eine spezifische, traumaassoziierte Hyperreaktivität bei der PTBS auslösen. Möglicherweise ist die Aktivierung durch die traumaassoziierten Stimuli so mächtig, dass der Organismus als Selbstschutz die gesamte Reagibilität herunterfährt. Folglich wären alle Reaktionen auf Stressoren gegenüber den Ausgangsbedingungen reduziert. Gleichzeitig könnte diese Verringerung der Stressreagibilität für den traumaassozierten Stress jedoch nicht ausreichen, um das gesteigerte Arousal auf einen Normalwert zu senken, sodass hier die Hyperreaktivität verbleibt, während ansonsten eine Hyporeaktivität besteht.

Der TPRI von gesunden Probanden wurde bereits mehrmals während einer Rechenphase aufgezeichnet. Oft wurde ein Anstieg des TPRI durch den Rechenstress berichtet, der als $\alpha$ adrenerge Aktivierung interpretiert werden kann (Winzer et al. 1999, Ring et al. 2000, Ring et al. 2002, Liu et al. 2007). Eine ähnliche Stressantwort des TPRI war folglich auch in dieser Studie erwartet worden. Im Follow-up blieb der TPRI jedoch weitgehend unverändert. Bereits in der Baseline-Messung war der TPRI im Mittel konstant geblieben, sodass sich in der ANOVA und graphisch bezüglich der Reactivity keine Veränderung zwischen den beiden Messzeitpunkten ergab. Interessanterweise verzeichnete auch die Kontrollgruppe nicht etwa einen Anstieg, sondern deskriptiv einen leichten Abfall des TPRI (Bornschein 2014). Neben den Arbeiten, die einen Anstieg des TPRI unter Rechenstress beschreiben, finden sich jedoch auch einige Studien, die wie in dieser Arbeit ein abweichendes Verhalten des TPRI feststellten. So fanden Bacon et al. (2010) und Paine et al. (2013) bei gesunden Probanden einen unveränderten bzw. geringfügig gesunkenen TPRI während eines Rechenstressors. Tsai et al. (2003) kamen zu einem ähnlichen Ergebnis, als sie einen stroop-color-and-word-Test als kognitiven Stress anwandten. In einer Studie mit 172 gesunden Probanden wurde der Messwert jeder Minute eines Rechentests einzeln ausgewertet. Es zeigte sich innerhalb der fünf Minuten zunächst ein Abfall des TPRI, gefolgt von einem Anstieg knapp über den Ausgangswert (Kelsey et al. 1999). Da in der vorliegenden Studie lediglich der Phasenmittelwert herangezogen wurde, könnten solche Effekte verborgen geblieben sein.

Es wird deutlich, dass der fehlende Anstieg des TPRI in dieser Studie kein Einzelfall ist. Neben der $\alpha$-adrenergen Steuerung wird auch der $\beta 2$-adrenerge Einfluss auf den TPRI als eine mögliche Erklärung für diese fehlende Reactivity des TPRI beschrieben (Kelsey et al. 1999, Sherwood et al. 1999, Ring et al. 2002, Paine et al. 2013). Hinweise für diese Hypothese lieferte eine Studie, in der Probanden einen Rechentest absolvierten, nachdem sie entweder einen unselektiven Betablocker (Propranolol) oder ein Placebo eingenommen hatten. Der TPRI stieg mit und ohne den Betablocker durch den Rechenstress an, mit dem Betablocker war der Anstieg jedoch leicht höher (Winzer et al. 1999). In einer ähnlich aufgebauten Studie wurde der Einfluss eines $\alpha_{1}$-Adrenozeptor-Antagonisten (Doxazosin) auf den TPRI getestet. Dabei war der Anstieg des TPRI als Reaktion auf den Rechenstress durch die Gabe des Doxazosin im Vergleich zur Placebo-Gruppe nicht abgeschwächt (Ring et al. 2000). Folglich können die durch Rechenstress induzierten TPRI-Veränderungen nicht rein $\alpha$-adrenerger 
Natur sein (Ring et al. 2002). Der fehlende Anstieg des TPRI könnte jedoch durch die Zunahme der $\beta 2$-adrenergen Aktivität und einer damit einhergehenden antagonistischen Wirkung auf eine mögliche $\alpha$-adrenerge Aktivierung erklärt werden. Der $\beta 2$-adrenerge Einfluss führt peripher, v. a. in den Muskeln, zu einer Vasodilatation (Joyner und Dietz 2003), sodass der TPRI sinkt, während der sBP durch ein erhöhtes HZV steigt. Dies ist durch die gesteigerte Durchblutung der Muskeln ein wichtiger Mechanismus der fight or flight-Reaktion.

Die gleichzeitige $\alpha$ - und $\beta$-adrenerge Aktivierung mit einem antagonisierenden $\beta$-adrenergen Anteil erscheint als Antwort auf den Rechenstress schlüssiger als ein unveränderter $\alpha$-adrenergen Tonus. Insbesondere auch da es gleichzeitig zu einem klaren Anstieg von HR und sBP kommt. Die inkonsistente Datenlage zu der Reaktivität des TPRI könnte dabei auch durch Geschlechter- und Altersunterschiede zustande kommen. Während bei Männern und älteren Frauen (postmenopausal) die $\alpha$-adrenerge Steuerung im Vordergrund steht, weisen aktuelle Forschungsergebnisse der $\beta 2$-adrenergen Modulation eine entscheidende Rolle in der Blutdruckregulation von jüngeren Frauen zu. Folglich korreliert der TPRI zwar mit der sympathischen Nervenaktivität von jungen Männern und postmenopausalen Frauen, bei jungen Frauen findet sich jedoch vermutlich aufgrund des entgegenwirkenden $\beta 2$-adrenergen Einflusses keine Korrelation (Briant et al. 2016, Joyner et al. 2016). Das Durchschnittsalter lag im Follow-up bei 45,7 Jahren, die älteste Patientin war 59 und die jüngste 28 Jahre alt. Somit ist es möglich, dass unter den Patientinnen unterschiedliche Regulationsmechanismen vorherrschten, je nachdem ob sie prä- oder postmenopausal waren. Mögliche Geschlechterund Altersunterschiede in der Stressadaptation des TPRI sollten daher Gegenstand zukünftiger Forschung sein, um ein besseres Verständnis der zugrundeliegenden Mechanismen zu ermöglichen. Zusammenfassend ist die aktuelle Studienlage zu der Reaktivität des TPRI auf das Rechnen als inhomogen einzustufen. Der TPRI verhält sich in ähnlichen Stresssituation auf sehr konträre Weise. Eine unterschiedlich ausgeprägte $\beta 2$-adrenerge Stressregulation ist eine mögliche Erklärung für die Inkonsistenzen. In jedem Fall zeigt sich jedoch keine Besserung der Rechnen-Reactivity des TPRI von Baseline zu Follow-up.

Die erwartete Verkürzung der PEP im Sinne einer $\beta$-adrenergen Aktivierung blieb im Follow-up aus. Stattdessen zeigte sich die PEP in der Rechenphase weitgehend unverändert zur Phase Ruhe 1. In der Baseline-Messung kam es zwar graphisch zu einer geringfügigen Verkürzung der PEP während des Rechens, in den ANOVA-Rechnungen findet sich jedoch kein signifikanter Zeiteffekt. Somit ist von einem unveränderten Befund der PEP im Followup auszugehen. Die Kontrollgruppe war dagegen durch eine deutlichere Abnahme der PEP gekennzeichnet. Eine Vielzahl an Studien zeichnete bereits die PEP von gesunden Probanden unter Rechenstress auf. Die Datenlage erscheint hier eindeutig und konsistent zur Kontrollgruppe: In allen uns bekannten Studien kommt es zu einer Abnahme der PEP durch den Rechenstress, in vielen Arbeiten zeigt sich dieser Effekt auch signifikant (Allen und Crowell 1989, Burns et al. 1992, Berntson et al. 1994, Montoya et al. 1997, Kelsey et al. 1999, Winzer et al. 1999, Ring et al. 2000, Mezzacappa et al. 2001, Ring et al. 2002, McGrath et al. 2005, Heponiemi et al. 2007, Kelsey et al. 2007, Ottaviani et al. 2008, Bacon et al. 2010, 
Chumaeva et al. 2010, Matsumura et al. 2012). Dagegen wurde das Verhalten der PEP unter Rechenstress unseres Wissens noch nicht bei Probanden mit PTBS untersucht, sodass hier Vergleichswerte fehlen. Lomb (2011) fand für ihre psychosomatische Kohorte ebenfalls eine Verkürzung der PEP unter Rechenstress. Dabei hatten die psychosomatischen Patienten graphisch jedoch eine geringere Reaktivität als die Kontrollgruppe - ein ganz ähnliches Ergebnis wie bei Bornschein (2014), die den PTBS-Patientinnen eine gegenüber der Kontrollgruppe signifikant niedrigere Reaktivität bescheinigte. Die fehlende Verkürzung der PEP könnte somit Ausdruck der allgemeinen Hyporeagibilität bei der PTBS sein. Da die PEP bereits in Ruhe 1 sehr niedrig war, wäre es auch denkbar, dass bereits vor der Rechenphase eine deutliche $\beta$-adrenerge Aktivierung vorlag. Die (vermutlich eingeschränkte) Stressregulationsfähigkeit der PTBS-Probanden könnte dabei nicht ausgereicht haben, um eine weitere Verkürzung der PEP beim Rechnen zu bewirken. Eine verfrühte Stressaktivierung erscheint aber aufgrund des Verhaltens der anderen Parameter eher unwahrscheinlich. Auch andere Erklärungsversuche wie dissoziative Zustände oder Ablenkungseffekte wirken in Zusammenschau mit den anderen Parametern wenig überzeugend. Die Sensitivitätsanalyse im Anhang deutet zudem nicht auf einen starken Einfluss der Betablockermedikation auf die PEP hin, auch wenn eine Konfundierung durch andere Medikamente natürlich nicht auszuschlieBen ist. Eine Hyporeagibilität der PEP bleibt daher ein möglicher Erklärungsansatz.

Durch die fehlende Verkürzung bildet die PEP im Follow-up nicht die $\beta$-adrenerge Aktivierung $\mathrm{ab}$, wie sie angesichts des Verlaufs der Herzrate und des systolischen Blutdrucks zu vermuten war. Dies deutet darauf hin, dass der Anstieg von HR und sBP vorwiegend durch ein Absinken des Parasympathikus zu erklären ist. Damit im Einklang steht das signifikante Sinken der BRS beim Rechenstress. Übereinstimmend hiermit konnten auch andere Studien eine vagale Aktivitätsminderung in Form einer gesunkenen BRS als Antwort auf Rechnen feststellen (Allen und Crowell 1989, Steptoe und Sawada 1989, Mezzacappa et al. 2001, Matsumura et al. 2012, Xie et al. 2017). Sahar et al. (2001) fanden weitere Hinweise, dass die kardiovaskuläre Antwort auf Rechenstress vorwiegend vagal moduliert sein könnte. Dabei ist die Abnahme der BRS in Baseline und Follow-up graphisch nahezu identisch, sodass sich die vagale Reaktivität zwischen den beiden Messzeitpunkten nicht gesteigert zu haben scheint. Eine Annäherung an die Kontrollgruppe kann somit nicht bilanziert werden, und es ist weiterhin von einer vagalen Hyporeaktivität auszugehen.

Diese Ergebnisse reihen sich ein in die aktuelle Literatur, die zunehmend dem Parasympathikus eine wichtige Rolle in der autonomen Dysfunktion der PTBS zuweist. Erste Arbeiten zur vagalen Reaktivität deuten übereinstimmend mit der vorliegenden Studie auf eine vagale Hyporeaktivität hin. So fanden Hughes et al. (2007) bei Frauen mit einer PTBS und/oder einer Depression ein signifikant geringeres Absinken der BRS während eines Ärgertest als bei einer Kontrollgruppe. Auch während verschiedener Affekte (positiv, negativ, neutral, traumaassoziiert) wurde für eine PTBS-Kohorte ein durchgängig niedrigeres vagales Aktivitätsniveau sowie eine starrere Stressregulation im Vergleich zu einer Kontrollgruppe beschrieben (Hauschildt et al. 2011). Das Berichten über ein traumatische Erlebnis konnte 
bei 9 PTBS-Probanden keine signifikanten Veränderungen in der HF-HRV auslösen, während bei einer gesunden Kontrollgruppe das Erzählen eines stressvollen Erlebnisses zu einem Absinken der HF-HRV führte (Cohen et al. 1998). Diese Ergebnisse konnten für weitere 14 PTBS-Probanden diesmal auch im Vergleich zu Probanden mit einer Panikstörung reproduziert werden (Cohen et al. 2000). Für Traumaexponierte war zudem eine hohe PTBS-Symptomatik mit einer geringen vagalen Reactivity während des Erinnerns eines traumatischen sowie eines angenehmen Erlebnisses assoziiert (Arditi-Babchuk et al. 2009). Eine Ausnahme bildeten jedoch Keary et al. (2009), die im Vergleich zu einer Kontrollgruppe eine erhöhte vagale Reaktivität bei PTBS-Probandinnen beschrieben und damit im Widerspruch zu den anderen Studien stehen. Dabei war es zu einem stärkeren Absinken der HF-HRV während eines Rechentests und während traumabezogenen Stresses gekommen. Der abweichende Befund von Keary et al. könnte eventuell seine Ursache in der Kontrollgruppe haben (Bornschein 2014). Denn 70 \% der Kontrollgruppe von Keary et al. (2009) hatten ebenfalls eine Traumatisierung erlebt. Bei Arditi-Babschuk et al. (2009) und Hauschildt et al. (2011) finden sich Hinweise, dass auch traumatisierte Probanden ohne diagnostizierte PTBS Veränderungen in der vagalen Regulation haben könnten. Die Mehrheit der uns bekannten Studien stimmt somit mit der vorliegenden Studie überein, indem sie auf eine Hyporeaktivität des Parasympathikus hinweist. Diese fand sich bei ganz unterschiedlichen Stressphasen - sowohl traumaassoziierte als auch traumaunabhängige Stressoren waren darunter. Im Gegensatz dazu steht die oben beschriebene stressorabhängige HR- und sBP-Reaktivität, denn bei traumaspezifischen Stressoren tritt in der Regel eine Hyperreaktivität und bei traumaunabhängigen Stressoren häufig eine Hyporeaktivität der beiden Parameter auf.

In beiden Fällen könnte jedoch eine verringerte vagale Regulation und Kontrolle einen verbindenden Mechanismus darstellen. Zum einen könnte eine mangelnde vagale Kontrolle kombiniert mit einer überschießenden Sympathikusreaktion bei traumaassoziierten Reizen eine Hyperreaktivität bewirken. Hierauf weist die Studie von Sack et al. (2004) hin, die bei PTBS-Probanden mit einem geringeren basalen Parasympathikustonus eine stärkere und länger andauernde HR-Antwort auf einen traumaassoziierten Stressor im Vergleich zu PTBSProbanden mit einem höheren basalen Parasympathikustonus nachweisen konnten. Zum anderen könnte eine verringerte vagale Stressregulation zusammen mit einer gleichzeitigen sympathischen Hyporeaktivität in einer allgemeinen Hyporeagibilität resultieren. Übereinstimmend hiermit beschränkte sich die in dieser Studie beobachtete Hyporeagibilität nicht nur auf das vagale System, sondern erstreckte sich gleichermaßen auch auf das sympathische System. Ob es sich hierbei um eine primäre sympathische Hyporeagibilität handelt oder aber diese allein durch eine reduzierte vagale Disinhibition verursacht wird, ist dabei unklar. Aber auch bei einer primären sympathischen Hyporeagibilität wäre die reduzierte vagale Disinhibition als verstärkender Faktor vermutlich maßgeblich beteiligt. In jedem Fall erscheinen die Vorgänge jedoch komplex und weitere Forschung ist für ein besseres Verständnis bezüglich des sympathisch-parasympathischen Zusammenspiels in der PTBS notwendig. 
Insgesamt ist ein Absinken des vagalen Tonus als entscheidender Mechanismus in der Reaktion auf den Rechenstress die überzeugendste Erklärung für die hier beschriebenen Befunde. Sowohl der Anstieg von HR und sBP als auch das Absinken der BRS stehen damit im Einklang. Eine $\beta$-adrenerge Aktivitätssteigerung kann sich zwar ebenfalls in einem Anstieg von HR und sBP äußern, scheint aber aufgrund der fehlenden Verkürzung der PEP im Follow-up nicht maßgebend in der beobachteten Stressregulation zu sein. In jeden Fall ist jedoch trotz der vermutlich vordergründig vagalen Modulation von einer geringen $\beta$ adrenergen Aktivierung durch den Wegfall des hemmenden Vagotonus auszugehen. Der ebenfalls weitgehend konstant bleibende TPRI festigt das Bild, hätte doch ein Absinken des TPRI als $\beta$-adrenerge Steigerung und ein Anstieg des TPRI als $\alpha$-adrenerge Steigerung gewertet werden können. Ein konstanter TPRI kann aber als kongruent mit einem vagalen Fokus in der Stressregulation angesehen werden. Es konnte zudem für die Reaktion auf den Rechenstress keine Veränderung zwischen Baseline und Follow-up festgestellt werden. Bei der verminderten Stressantwort auf das Rechnen scheint es sich um ein auch über Jahre robustes, schwierig veränderliches Kennzeichen der PTBS zu handeln.

\subsubsection{Babyschreien}

Der Stressor Babyschreien wurde unseres Wissens in der Baseline-Untersuchung dieser Studie erstmals bei einer PTBS-Kohorte verwendet. Er wurde jedoch bereits bei gesunden Probanden sowie bei Studien zu Kindesmissbrauch eingesetzt, hier erfolgte allerdings bislang nur selten eine multidimensionale Betrachtung der Reactivity auf das Babyschreien. In der Baseline-Messung überraschte das Babyschreien, indem es bei der gesunden Kontrollgruppe zu einem grundsätzlich anderen Reaktionsmuster als der Rechenstress führte. Es kam zu einer vorwiegend vagalen Aktivitätssteigerung, die sich u. a. in einem deutlichen Anstieg der BRS zeigte. HR und sBP sanken etwas, während TPRI und PEP sich weitgehend unverändert präsentierten. Hieraus folgerte Bornschein, dass das sympathische System kaum auf das Babyschreien reagiert hatte. Sie sieht als eine mögliche Interpretation für das beobachtete Reaktionsmuster einen vagalen Anstieg im Zuge einer Aktivierung des Bindungssystems (Bornschein 2014). Das Bindungssystem (s. Kap. 1.2.1) ist eng mit dem Parasympathikus verbunden und wird durch distress vocalizations wie dem Babyschreien aktiviert. Es handelt sich um einen uralten Mechanismus, durch den die Mutter angehalten werden soll, ihrem hilflosen Kind beizustehen. Aber auch bei der Herdenbildung und in diesem Zuge auch bei sozialen Interaktionen im Erwachsenenalter spielt das Bindungssystem eine Rolle, sodass das Babyschreien als sozialer Stressor angesehen werden kann (Panksepp 1998, Sachsse 2003).

Wie schon bei der Antwort auf den Rechenstress offenbarte sich auch bei der Reaktion auf das Babyschreien eine Hyporeagibilität bei den PTBS-Probandinnen der Baseline-Messung. Während jedoch beim Rechenstress vagaler Abfall und sympathischer Anstieg verringert waren, ist beim Stressor Babyschreien der bei Gesunden beobachtete Anstieg des Parasympathikus reduziert. Das parasympathische System scheint somit bei verschiedenen Stressreaktionen und sowohl bei Anstieg als auch bei Abfall hyporeagibel zu sein. So konnte gezeigt 
werden, dass die dysfunktionale vagale Stressregulation nicht nur bei traumassoziierten Reizen (Cohen et al. 1998, Cohen et al. 2000, Arditi-Babchuk et al. 2009) und Ärgerreaktionen (Hughes et al. 2007), sondern ebenso bei kognitivem und sozialem Stress bei der PTBS eine entscheidende Rolle spielt (Meyer et al. 2016). Die Hyporeagibilität äußerte sich durch größtenteils unveränderte kardiovaskuläre Parameter - HR, sBP, PEP und TPRI blieben weitgehend unbeeinflusst von der Stressphase Babyschreien. Die BRS stieg zwar leicht an, dieser Anstieg war jedoch signifikant geringer als bei der Kontrollgruppe (Bornschein 2014).

Diese Hyporeagibilität bestand auch im Follow-up, wobei die Reaktion der PTBS-Patientinnen auf das Babyschreien größtenteils deckungsgleich mit dem Baseline-Befund war und keine Annäherung an die Kontrollgruppe stattfand. Im Einzelnen zeigte sich in der graphischen Analyse ein nahezu identisches Verhalten für HR, sBP, TPRI und BRS an den beiden Messzeitpunkten. Auch in der ANOVA konnten keine signifikanten Veränderungen nachgewiesen werden. Für den TPRI ist zwar ein signifikanter Zeiteffekt zu beschreiben, der aber auf unterschiedlichen absoluten Werten während der gesamten Messung und nicht auf einer veränderten Reactivity zu beruhen scheint (s. Kap. 5.3.4). Die unveränderte BRS-Antwort erscheint besonders aussagekräftig, ist doch der Anstieg der BRS im Zuge einer Parasympathikusaktivierung die Schlüsselreaktion der Kontrollgruppe. Ebenso zeigte sich an dieser Stelle die Hyporeagibilität der PTBS durch einen signifikant niedrigeren Anstieg der BRS besonders deutlich. Die PEP verhielt sich als einziger Parameter im Follow-up graphisch gegensätzlich zur Baseline-Messung. Sie verlängerte sich durch das Babyschreien, während sie sich beim Zeitpunkt Baseline noch verkürzte und in der Kontrollgruppe weitgehend konstant blieb bzw. sich geringfügig verlängerte. Zu beachten ist hierbei, dass die verschiedenen phasenabhängigen Reaktionen der PEP allesamt nicht signifikant waren, auch ein Zeiteffekt fand sich nicht. Es besteht aber eine signifikante Wechselwirkung zwischen Stressorwirkung und Messzeitpunkt. Grundsätzlich könnte die angestiegene PEP als Zeichen einer verringerten $\beta$-adrenergen Aktivierung eine stärkere Entspannung während des Babyschreiens anzeigen. Aufgrund der schwierig einzugrenzenden Wechselwirkungen und dem nicht übereinstimmenden Verhalten der anderen Parameter, erscheint diese Interpretation aber fraglich.

Denkbar ist, dass die Patientinnen durch die häufig bereits in der Kindheit erfolgten Traumatisierungen eine Störung des Bindungssystems aufweisen. Die verminderte Reaktion auf das Babyschreien könnte dann einer Dysfunktion der physiologischen Regulation des Bindungssystems entsprechen, z. B. im Sinne einer geringeren Sensitivität auf die distress vocalizations und damit auch auf die Hilfsbedürftigkeit des Kindes. Während sich viele Studien der autonomen Reaktion auf Babyschreien bei misshandelnden Müttern widmeten, ist wenig dazu bekannt, wie sich Babyschreien bei in der Kindheit misshandelten Erwachsenen auswirkt. Gerade diese Personengruppe könnte aber Hinweise auf die Auswirkungen eines dysfunktionalen Bindungssystems im Erwachsenenalter liefern. Casanova et al. (1994) spielten 13 Müttern mit physischen Misshandlungen in der Kindheit Videoaufnahmen von einem weinenden und lächelnden Säugling vor. Während bei den Aufnahmen von dem weinenden Kind die Hautleitfähigkeit unverändert blieb, stieg sie bei Betrachtung des lächelnden Kindes 
an. Im Gegensatz dazu verzeichnete eine Kontrollgruppe (Mütter ohne physische Misshandlungen in der Vorgeschichte) einen Anstieg der Hautleitfähigkeit bei dem weinenden Kind, aber nicht bei dem lächelnden Kind. Bezüglich der Herzrate und der von den Probandinnen beschriebenen Emotionen unterschieden sich die beiden Gruppen dagegen nicht (Casanova et al. 1994). Auch wenn die sympathische Aktivierung der Kontrollgruppe der vorliegenden Studie widerspricht, stimmt die verminderte Reaktion der traumatisierten Mütter bei dem Babyschreien mit dieser überein. Dass sich stattdessen eine Reaktion bei dem lächelnden Baby zeigte und folglich keine allgemeine Hyporeagibilität vorherrschte, stellt einen zusätzlichen Hinweis auf ein gestörtes Bindungssystem als mögliche Genese dar. Da jedoch nur wenige Parameter erfasst wurden und nicht bekannt ist, ob einige der Probandinnen auch an einer PTBS litten, ist eine genaue Interpretation schwierig. Rejmann et al. (2014) erhoben zwar mehr Parameter (HR, PEP, Hautleitfähigkeit und RMSSD), untersuchten aber primär die Psychophysiologie von misshandelnden Müttern. Gleichzeitig wurde jedoch auch erfasst, ob die Probandinnen Misshandlungen in der Kindheit erlebt hatten. Diese Kindheitstraumatisierungen hatten jedoch keinen oder höchstens geringfügigen Einfluss auf die physiologischen Reaktionen der misshandelnden Mütter oder der Kontrollgruppe (nicht misshandelnde Mütter) während des Babyschreiens. Eine Erfassung möglicher PTBS-Erkrankungen erfolgte nicht (Reijman et al. 2014). Weitere Studien sind somit notwendig, um mögliche stressphysiologische Auswirkungen eines gestörten Bindungssystems zu identifizieren. Es sollte in jedem Fall auch eine Unterscheidung in kindheitstraumatisierte Probanden mit und ohne PTBS erfolgen, um zu klären, ob spezifische physiologische Symptome eines gestörten Bindungssystems existieren oder die bei Casanova et al. (1994) beobachteten Auffälligkeiten durch eine mögliche PTBS-Erkrankung mancher Probandinnen zustande kamen.

Denn die reduzierte Reaktivität der PTBS-Patientinnen könnte andererseits auch im Zuge der allgemeinen Hyporeagibilität der PTBS auftreten. Sie wäre dann nicht spezifisch mit dem Stressor Babyschreien und damit vermutlich mit dem Bindungssystem verbunden, sondern wäre auch bei allen anderen Stressoren zu beobachten. Da die Hyporeagibilität nach dem aktuellen Kenntnisstand zu einem bedeutenden Anteil vagal vermittelt wird, würde es nicht wundern, dass sie bei einem vorwiegend vagal wirksamen Stimulus wie dem Babyschreien besonders augenscheinlich wird. Für diese Theorie sprechen beispielsweise die in dieser Studie während der Phase Rechnen und sowie die von Hughes et al. (2007) während eines Ärgertests beobachtete vagale Hyporeagibilität bei der PTBS.

Albrecht hatte zudem die Frage aufgeworfen, ob nicht auch die häufige depressive Komorbidität bei den PTBS-Patientinnen die verminderte Reaktivität auf das Babyschreien mitbedingt haben könnte (Albrecht 2013). Hinweise hierfür hatte sie bei Riem et al. gefunden, die abhängig vom Oxytocin-Genotyp eine geringere HR-Reaktivität auf Babyschreien bei Probandinnen mit Depressivität beschrieben hatten (Riem et al. 2011). Die Ergebnisse unseres Followups sprechen jedoch eher gegen diese Hypothese. Obwohl die Depressivität im BDI signifikant sank, blieb die Hyporeagibilität der Patientinnen weitgehend unverändert. Auch konnte keine Korrelation zwischen den $\Delta \mathrm{R}$-Scores für das Babyschreien und der Differenz des BDI 
gefunden werden. Dafür könnte die depressive Symptomatik eventuell einen Einfluss auf die Rechen-Reactivity haben, wie in Kapitel 5.4 diskutiert wird.

Auch die dissoziative Symptomatik sieht Albrecht (2013) als möglichen Faktor in der Genese der Hyporeagibilität. Da der FDS im Mittel konstant blieb und zu beiden Messzeitpunkten einen ähnlich pathologischen Wert verzeichnete, kann ein eventueller Einfluss durch die Dissoziation auch mit den Erkenntnissen des Follow-ups nicht genauer differenziert werden. Zwar zeigte sich in Kapitel 5.4 keine Korrelation zwischen den Differenzen des FDS und den $\Delta$ R-Scores für das Babyschreien. Es ist jedoch weiterhin vorstellbar, dass das Babyschreien dissoziative Zustände bei einigen Patientinnen triggerte und sich so eine im Mittel verminderte Reaktivität zeigte. Hiermit übereinstimmend konnten Sack et al. (2012) eine verringerte HR-Reaktivität sowie eine verminderte vagale Reagibilität bei traumatisierten Probanden mit dissoziativen Zuständen nachweisen, als sie diese traumaassoziiertem Stress aussetzten. Auch wenn die Datenlage teilweise inkonsistent ist, gibt es mehrere Studien, die ebenfalls eine verminderte Reaktivität bei Probanden mit Dissoziation beschrieben (McDonagh-Coyle et al. 2001, Lanius et al. 2002, Koopman et al. 2004, Ebner-Priemer et al. 2005, Lanius et al. 2006, Sack et al. 2012). Die komplexen Traumafolgestörungen gehen häufig mit der Dissoziation einher und es wird diskutiert, ob es bei der PTBS einen Subtyp mit vorwiegend dissoziativem Verhalten zu unterscheiden gilt (Lanius et al. 2006, Sack et al. 2012). Für die Klärung der Frage, inwieweit die Hyporeagibilität bei dem Babyschreien auf die Dissoziation zurückzuführen ist, sind daher größere Stichproben mit einer Subgruppenbildung notwendig.

Die zu beiden Messzeitpunkten beobachtete Hyporeagibilität wird dadurch unterstrichen, dass der Stressor Babyschreien nur auf den TPRI einen signifikanten Phaseneffekt ausübte, auf alle anderen Parameter ergab sich kein Einfluss. Dies steht im Gegensatz zu dem Rechnen, das sich z. T. hochsignifikant auf die Parameter HR, sBP und BRS ausgewirkt hatte. Interessant ist diese ungleiche Reaktion auf die beiden Stressphasen v. a. auch, da das subjektive Stressempfinden in den beiden Phasen im Mittel etwa gleich hoch war. Im Post-hocTest der ANOVA zum Stressempfinden fand sich dementsprechend auch kein signifikanter Unterschied zwischen den beiden Stressoren. Obwohl die Patientinnen auf den Rechenstress physiologisch deutlich stärker reagierten, empfanden sie somit den Stress in den beiden Phasen als gleich hoch. Dies zeigt, dass die mentale und die physiologische Stressreaktion unabhängig voneinander ablaufen und diese beiden Achsen nicht immer im Einklang stehen. Eine ähnliche Diskrepanz entdeckten auch Pineles et al. (2013). Während sowohl das Stressempfinden bei traumaassoziiertem Stress als auch bei traumaunabhängigem Stress einen signifikanten Prädiktor für die Diagnose PTBS darstellte, war nur die physiologische Reaktion auf den traumaassoziierten, aber nicht auf den traumaunabhängigen Stressor ein signifikanter Prädiktor. Zudem zeigte das Stressempfinden für beide Stressoren eine sehr ähnliche Sensitivität für die PTBS, während der traumaunabhängige Stressor eine deutliche geringere Sensitivität bezüglich der physiologischen Reaktivität aufwies als der traumaassoziierte Stressor. Pineles et al. vermuten, dass das subjektiv erhöhte Stressempfinden eng mit negativen 
Affekten assoziiert ist. Negative Affekte finden sich nicht nur bei der PTBS, sondern treten bei allen Angsterkrankungen und affektiven Störungen auf. Dies wäre im Einklang mit der Beobachtung, dass sich die Reactivity zwar im Vergleich zum Stressempfinden durch eine geringere Sensitivität auszeichnete, die Spezifität jedoch, insbesondere bezogen auf traumaassoziierte Stressoren, deutlich höher war (Pineles et al. 2013). Weitere Forschungsarbeit ist notwendig, um das Verhältnis zwischen subjektivem und physiologischem Stress genauer zu charakterisieren. Dies ist auch für die Diagnose PTBS entscheidend - schließlich wird das berichtete Stressempfinden traditionell für die Diagnosestellung herangezogen.

Zusammenfassend konnte die Reaktion der PTBS-Patientinnen in der Baseline-Messung durch das Follow-up reproduziert werden - eine Annäherung an die Kontrollgruppe entsprechend den Hypothesen fand nicht statt. Als wahrscheinlichste Erklärung für die abgeschwächte Reaktion der PTBS-Patientinnen auf das Babyschreien erscheint die allgemeine vagale Hyporeagibilität der PTBS, die sich unspezifisch bei verschiedenen Stressoren präsentiert. Aber auch eine Dysfunktion des Bindungssystems oder dissoziative Zustände der Patientinnen stellen mögliche Erklärungsmodelle dar.

\subsubsection{Recovery}

Eine verspätete bzw. verminderte vagale Recovery-Regulation steht in Verdacht, mit einem erhöhten kardiovaskulären Risiko einherzugehen (Cole et al. 1999, Mezzacappa et al. 2001). Gleichzeitig fand Pole Anzeichen, dass der Recovery-Reaktion in der Stressphysiologie der PTBS eine besondere Bedeutung zukommen könnte, und erhoffte sich durch die Erforschung der Recovery ein besseres Verständnis von psychophysiologischen Vorgängen bei der PTBS (Pole 2007). An dieser Stelle knüpft das folgende Kapitel an.

Während sich eine Vielzahl von Studien auf die Reactivity bei der PTBS konzentrierte, war die Recovery bislang selten Gegenstand gezielter Forschung. Im Jahr 2002 verglichen Beckham et al. in einer der ersten Studien zu dieser Thematik männliche Kriegsveteranen mit und ohne PTBS bezüglich einer 90 Sekunden-Recovery-Phase nach einem Ärgertest. Dabei blieb der diastolische Blutdruck der PTBS-Gruppe während der Recovery erhöht, während er bei der Gruppe ohne PTBS zurück auf das Niveau eine Minute vor dem Ärgertest absank. Gleichzeitig waren die Kriegsveteranen mit PTBS durch eine stärkere Reaktivität des dBP bei dem Ärgertest aufgefallen (Beckham et al. 2002). Sack et al. entdeckten 2004, dass PTBSProbanden mit einem niedrigen basalen RSA-Wert im Vergleich zu PTBS-Probanden mit einem höheren basalen RSA-Wert mit einer verlängerten HR-Antwort auf einen traumassoziierten Stressor und einer signifikant längeren half-recovery-Zeit der HR reagierten. Die half-recovery-Zeit der HR war zudem negativ korreliert mit dem basalen RSA-Wert der Probandinnen und damit mit dem Parasympathikustonus (Sack et al. 2004). Für die Dauer der HR-Recovery nach akustischen Schreckreizen konnte zudem eine positive Korrelation mit der Schwere der PTBS-Symptomatik bei 29 Kriegsveteranen nachgewiesen werden. Dagegen korrelierte die PTBS-Schwere weder mit der Reaktivität noch mit der Habituation 
noch mit der Recovery von EMG und Hautleitfähigkeit (Kibler und Lyons 2004). Damit übereinstimmend wurde eine negative Korrelation zwischen der PTBS-Symptomlast bei traumaexponierten Probanden und dem vagalen Tonus während der Recovery nach einem traumaassoziierten Stressor beschrieben (Arditi-Babchuk et al. 2009). Lindauer et al. konnten dagegen keine signifikanten Unterschiede zwischen PTBS-Probanden und einer traumaexponierten Kontrollgruppe ohne PTBS bezüglich mehrerer vierminütiger Recovery-Phasen nach neutralen, stressreichen und traumaassoziierten Skripten feststellen (Lindauer et al. 2006).

Diese Studien sind, mit Ausnahme von Lindauer et al. (2006), erste Hinweise auf eine pathologische Recovery bei der PTBS. Bislang ist die Recovery bei der PTBS jedoch wenig verstanden. Dies liegt zum einen an der geringen Zahl an Studien mit zudem teilweise inkonsistenten Ergebnissen. Zum anderen zeigt sich an den oben genannten Beispielen, dass die Recovery auf ganz unterschiedliche Art evaluiert werden kann, sodass ein Vergleich verschiedener Studien oft schwierig ist. Unterschiede bei Probanden, Stressoren oder Parametern können einen erheblichen Einfluss auf die Recovery haben. Zudem kommen ganz verschiedene Zeitpunkte und Zeiträume für die Erfassung der Recovery in Frage. Und schließlich kann die Recovery sowohl anhand ihres Ausmaßes als auch anhand ihrer „Schnelle“ auf ganz unterschiedliche Weisen bestimmt werden. Dieser Problematik kann nur mit weiterer Forschungsarbeit begegnet werden; die vorliegende Studie ist ein Beitrag dazu.

Für die Evaluation der Recovery wurde die Phase Entspannungsmusik ausgewählt. Zwischen ihr und dem Babyschreien liegt die fünfminütige Ruhe 3-Phase und zu der Phase Rechnen beträgt der Abstand etwa eine Viertelstunde inklusive des Stressors Babyschreien. Mit der Wahl der Phase Musik verbunden ist die Entscheidung für die „mittelfristige“ Recovery. Bei der alternativen Möglichkeit der Evaluation der „kurzfristigen“ Recovery (die ersten Minuten nach der jeweiligen Stressphase) steht in der Regel die Frage nach einer verzögerten Recovery im Vordergrund. Diese Vorgehensweise findet sich bei Albrecht und Bornschein, somit sei für eine Analyse der Phasen Ruhe 2 und Ruhe 3 auf diese beiden Dissertationen verwiesen (Albrecht 2013, Bornschein 2014). Bei der „mittelfristigen“ Recovery steht eher das Ausmaß der Recovery im Fokus. Erreichen die PTBS-Patientinnen das Ruheniveau vor dem Stresstest und finden ähnliche Regulationsprozesse wie bei der Kontrollgruppe statt? Oder verbleiben sie auch einige Minuten nach der Stressphase auf einem erhöhten Stressniveau? Ein Vorteil, aber auch gleichzeitig ein Nachteil der Phase Musik als Recovery ist, dass mit ihr die over-allRecovery des gesamten Stresstests abgebildet wird. Sie kann nicht einem bestimmten Stressor zugeordnet werden. Dafür sind die Patientinnen jedoch nicht mehr in der Erwartungshaltung einer erneuten Stressphase und eine tatsächliche Entspannung kann theoretisch eintreten - falls dies nicht durch das Krankheitsbild der PTBS verhindert wird.

Es ist uns bislang nur eine Studie bekannt, die die Recovery der PTBS länger als fünf Minuten betrachtete. Keary et al. (2009) führten eine 10-minütige Recovery nach einem Rechentest bei einer PTBS- und einer Kontrollkohorte durch. Für die PTBS-Kohorte ergaben sich deskriptiv allenfalls geringfügige Unterschiede zu der Recovery der Kontrollgruppe, wobei 
eine detaillierte Analyse aufgrund eines anderen Fokus in der Studie fehlt (Keary et al. 2009). Zwei weitere Studien maßen zwar die Recovery-Reaktion anhand der Zeit, die benötigt wurde, um die Ruhewerte vor der Stressmessung zu erreichen. Es ist jedoch keinesfalls gesagt, dass damit die Recovery-Reaktion beendet war, denn auch eine Überkompensation ist denkbar. Regulationsvorgänge könnten so bislang verborgen geblieben sein. Daher soll in diesem Kapitel eine Recovery-Betrachtung über fünf Minuten hinaus vorgenommen werden.

In der Phase Entspannungsmusik wurde den Patientinnen mit der Mondscheinsonate von Beethoven eine als entspannend einzuordnende klassische Musik über Kopfhörer vorgespielt. Dem Einfluss von Musik auf Psyche und Physiologie widmet sich eine erhebliche Anzahl an Arbeiten. Albrecht und Bornschein geben beide bereits einen Überblick über den Forschungsstand bezüglich der Wirkung von Musik auf das kardiovaskuläre System inklusive einer Einordnung der Phase Musik in diesen Kontext (Albrecht 2013, Bornschein 2014). Da der Fokus dieses Kapitels auf der Recovery liegt, wird an dieser Stelle auf eine Wiederholung einer weitreichenden Analyse verzichtet. Es sei jedoch kurz angemerkt, dass die vielen verschiedenen Studien ein sehr inhomogenes Bild zum physiologischen Einfluss von Musik zeichnen. So konnte für Musik sowohl eine entspannende als auch eine aktivierende Wirkung nachgewiesen werden. Die Art der Musik und ihre emotionale Bewertung scheinen dabei entscheidende Faktoren darzustellen. Dies erklärt auch die Inkonsistenz der bisherigen Studien - die aktuelle Studienlage geht mit einer großen Bandbreite an unterschiedlicher Methodik, Untersuchungssituationen und verwendeter Musik einher (Koelsch und Jäncke 2015).

Für die Betrachtung der „mittelfristigen“ Recovery wurde ein Vergleich der Phasen Ruhe 1 und Entspannungsmusik in einer ANOVA für Messwiederholung gewählt (s. Kap. 4.2.4). Dies bietet den Vorteil, dass nicht die von dem Stressor Babyschreien beeinflusste Phase Ruhe 3, sondern die kardiovaskuläre Ruheaktivierung zu Anfang der Untersuchung und vor der Stresstestung als Äquivalenzmaß dient. Zudem charakterisierten Mezzacappa et al. (2001) eine suffiziente Recovery durch eine vagale Überkompensation. Diese Ansicht resultierte ebenfalls aus einer Gegenüberstellung der Werte der Recovery-Phase mit denen einer Ruhephase zu Beginn der Messung (Mezzacappa et al. 2001).

Eine überschießende Regulation der Recovery kann auch bei der PTBS-Kohorte festgestellt werden. So war die HR höchstsignifikant niedriger während der Musik als noch in der Phase Ruhe 1. Diese Veränderung zeigte sich in der Sensitivitätsanalyse (s. Anhang, Kap. 8.6) weiterhin hochsignifikant und auch die Effektstärke war unabhängig von dem Ausschluss der drei Patientinnen mit Wechsel in der Betablockermedikation hoch $\left(\eta^{2}=0,47\right.$ gegenüber $\eta^{2}$ $=0,43$ nach Ausschluss der drei Patientinnen). Somit kann der Einfluss der Betablockermedikation an dieser Stelle vernachlässigt werden. Der Umstand, dass die HR über die Ruhewerte vor der Stresstestung hinaus absinkt, wurde auch für die Recovery von Gesunden berichtet (Mezzacappa et al. 2001, Hamer und Steptoe 2007). Bei Ring et al. (2002) ist zumindest anhand der aufgeführten Graphiken ein ähnlicher Effekt anzunehmen. Die BRS war in der Phase Musik signifikant höher als in Ruhe 1 - ein Befund der die Hypothese einer 
vagalen Überkompensation in der Recovery von Mezzacappa et al (2001) stützt. Diese hatten im Vergleich zu der Ruhephase vor der Stresstestung ebenfalls eine höhere BRS sowie einen höheren RMSSD in der Recovery-Phase entdeckt. Ein höherer RMSSD-Wert in der Recovery-Phase kann bei Ring et al. (2002) wiederum anhand der Graphik vermutet werden und Hamer und Steptoe (2007) berichteten ebenfalls von einer im Vergleich zu der anfänglichen Ruhephase angestiegenen HR-Variabilität während der Recovery. Erwähnenswert ist auch, dass die BRS deskriptiv in der Phase Musik den höchsten Wert der ganzen Messung erreicht.

Bei Mezzacappa et al. (2001) zeigte die PEP entgegen der HR und den vagalen Parametern eine geringere Entspannungsreaktion in der Recovery an, indem sie sich nach dem Stress kürzer als davor präsentierte. Diese Beobachtung führte u. a. zu der Aussage, dass die Recovery vorwiegend vagal bei gleichzeitig anhaltender sympathischer Aktivierung moduliert sein müsste. Die vorliegende Studie spricht dagegen der $\beta$-adrenergen Aktivitätsabnahme ebenfalls einen Anteil an der Regulierung der Recovery zu. So ist die PEP genau wie die BRS in der Phase Musik signifikant höher als in Ruhe 1, der TPRI hatte sogar hochsignifikant höhere Werte während der Musik. Wie in Kapitel 5.3.2.1 diskutiert, ist der Anstieg des TPRI mit einer Abnahme der $\beta$-adrenergen Aktivierung vereinbar. So könnte durch die $\beta$-adrenerge Aktivitätsabnahme der $\alpha$-adrenerge Einfluss auf den TPRI überwiegen und zum Anstieg führen. Ähnliche Resultate fanden auch Ring et al. (2002) für PEP und TPRI sowie Hamer und Steptoe (2007) für den TPRI. Ein Grund für die unterschiedlichen Ergebnisse in Vergleich zu Mezzacappa et al. (2001) könnte die Länge des betrachteten RecoveryZeitraums sein.

Einzig der sBP unterschied sich in der Phase Musik nicht signifikant von der Phase Ruhe 1. Er war zu beiden Messzeitpunkten sogar höher in der Phase Musik und erscheint damit einer Entspannungsreaktion entgegengesetzt. Bei der Sensitivitätsanalyse (s. Anhang, Kap.8.6) wird dieser Trend auch signifikant, sodass der Effekt eventuell durch die Einnahme der Betablocker verringert wurde. Aber auch nach Ausschluss der drei Patientinnen mit veränderter Betablockermedikation ist die Effektstärke mit $\eta^{2}=0,18$ die geringste unter den bislang beschriebenen Parameterunterschieden zwischen Ruhe 1 und Musik. Tatsächlich stimmt jedoch der Befund eines im Vergleich zur anfänglichen Ruhephase höheren sBP in der Recovery mit verschiedenen Studien bei Gesunden überein (Gillin et al. 1996, Mezzacappa et al. 2001, Ring et al. 2002, Tsai et al. 2003, Hamer und Steptoe 2007, Matsumura et al. 2012). Betrachtet man den unveränderten sBP in Zusammenschau mit dem Anstieg des TPRI und dem Sinken von PEP und HR, muss es das $\alpha$-adrenerge System sein, das einer Abnahme des sBP entgegenwirkt bzw. sogar den Trend zum Anstieg bewirkt. Dies führt zu der Vermutung, dass das $\beta$-adrenerge System eine bessere bzw. schnellere Recovery als das $\alpha$-adrenerge System zeigt. Dabei könnte die eigentliche Stressreaktion des $\alpha$-adrenergen Systems durch die gleichzeitige $\beta$-adrenerge Aktivierung bei dem Parameter TPRI zunächst verborgen geblieben sein und sich nun im Vergleich zu der $\beta$-adrenergen Stressreaktion deutlich langsamer normalisieren. Andererseits könnte es sich auch um eine überschießende $\alpha$-adrenerge Gegenregulation handeln, die eine Reaktion auf die $\beta$-adrenerge Abnahme darstellt und nicht im 
direkten Zusammenhang mit den Stressoren und einer $\alpha$-adrenergen Recovery steht. Diese Gegenregulation könnte der Verhinderung von zu starken Blutdruckschwankungen dienen.

Bei dem Vergleich der Phasen Musik und Ruhe 1 gilt zu beachten, dass sowohl Baseline als auch Follow-up in die Rechnung miteingingen. Ein Zeiteffekt ergab sich jedoch wie schon bei den Reactivity-ANOVAs nur für den TRPI - vermutlich aufgrund der während der ganzen Messung erhöhten TPRI-Werte im Follow-up. Neben einer Überkompensation im Sinne einer regelrecht überschießenden Entspannungsreaktion kommt auch ein erhöhtes Ruheaktivierungsniveau in der Phase Ruhe 1, beispielsweise im Zuge der Anspannung aufgrund der beginnenden Untersuchung, als Erklärung in Frage. In beiden Fällen spricht die deutliche Entspannungsreaktion in der letzten Phase der Messung jedoch für eine erfolgreiche Erholung von dem Stress. Insgesamt erscheint die mittelfristige Recovery-Reaktion der PTBSPatientinnen im Vergleich zu Gesunden typisch zu verlaufen, pathologische Regulationsvorgänge konnten nicht identifiziert werden. Es kommt zu einer vagalen Tonussteigerung sowie zu einer $\beta$-adrenergen Aktivitätsminderung. Die $\alpha$-adrenerge Aktivierung scheint dagegen nicht zu sinken, sondern eher zu steigen. Zudem ist auch mehrere Minuten nach den Stresstests eine Überkompensation zu beobachten, die jedoch auch auf einem erhöhten Stressniveau zu Beginn der Untersuchung beruhen könnte.

Wie im Kapitel 5.3.2.2 beschrieben führt das Babyschreien zu keiner klassischen fight or flightReaktion. Doch wie unterscheiden sich Rechnen und Babyschreien, wenn man sie in Bezug zu der Phase Musik setzt, die wie soeben gezeigt mit einer deutlichen Entspannung einhergeht? Hierfür werden die beiden Recovery-ANOVAs (s. Kap. 4.3.6) herangezogen, die sowohl Baseline als auch Follow-up einschließen. Das Rechnen unterschied sich von der Musik deutlich, es ließen sich für alle Parameter signifikante (sBP), hoch signifikante (PEP) bzw. sogar höchst signifikant (HR, TPRI, BRS) Recovery-Reaktionen berechnen. Dies steht im klaren Gegensatz zu der Tatsache, dass die Phasen Musik und Babyschreien keinen einzigen signifikanten Unterschied aufwiesen. Fast wirkt es als ob das Babyschreien zu einer Entspannung geführt hätte, wäre da nicht das von den Patientinnen berichtete hohe subjektive Stressempfinden. Das Stressempfinden während Rechnen und Babyschreien erreichte ähnlich hohe Werte, während das Stressempfinden der Phase Musik deutlich geringer angegeben wurde und zumindest im Follow-up mit der Ruhe 1 vergleichbar war. Der Kontrast zwischen Psyche und Physiologie wurde bereits in Kapitel 5.3.2.2 diskutiert. An dieser Stelle soll die Diskussion um die Erkenntnis ergänzt werden, dass sich das Babyschreien entgegen dem Empfinden der Patientinnen wenig von der physiologischen Entspannungsreaktion in der Phase Musik unterscheidet - ein aus unserer Sicht bemerkenswerter Befund. Dies führt jedoch auch zu der Frage, inwieweit die betrachtete over-all-Recovery schon in der Phase Babyschreien begann bzw. inwieweit sich die Regulationsprozesse dieser beiden Phasen gegenseitig beeinflussten. Schließlich lag das Babyschreien zwischen dem Rechnen und der Musik und ist rechnerisch kaum von der eigentlichen Recovery unterscheidbar. Für eine differenzierte Betrachtung eines möglichen Effekts sollte in zukünftigen Studien die Reihenfolge der Stressphasen Rechnen und Babyschreien rotiert werden. Zudem könnte es interes- 
sant sein, längere Phasen für das Babyschreien und die Recovery auszuwählen. Hierdurch könnte ein eingehenderes Verständnis dafür erlangt werden bezüglich welcher Regulationsprozesse sich Babyschreien und Musik unterscheiden bzw. überschneiden.

Wie schon in der ANOVA mit der Gegenüberstellung von Ruhe 1 und Musik fanden sich in den Recovery-ANOVAs lediglich signifikante Zeiteffekte für den TPRI, die wiederum auf den während der gesamten Follow-up-Messung erhöhten TPRI-Werten und nicht auf einer veränderten Recovery zu beruhen scheinen. Es kann somit keine Veränderung der Recovery im Vergleich Baseline und Follow-up bilanziert werden.

\subsubsection{Allgemeine Stressreagibilität sowie Charakteristika der einzelnen Parameter}

In diesem Kapitel sollen die allgemeine Reagibilität und der Verlauf bzw. die Charakteristika der einzelnen Parameter betrachtet werden. Es werden deskriptive Ergebnisse analysiert, die sich aus den Graphen in Kapitel 4.3.3 sowie aus den Recovery- und $\Delta$ R-Scores ergeben.

Die HR-Graphen von Follow-up und Baseline verlaufen annähernd gleich. Der Graph der Kontrollgruppe zeigt dagegen deutlich größere Ausschläge, über die ganze Untersuchung hinweg erscheint die Reagibilität hier höher. Die $\Delta \mathrm{R}$-Scores bestätigen den graphischen Eindruck und weisen entgegen Hypothese 3b keine erhöhte Recovery und damit Reagibilität im Follow-up nach. Dabei bescheinigt die Literatur der HR eine besondere Eignung darin, Entwicklungen im ANS der PTBS abzubilden (Griffin et al. 1997, Rabe et al. 2006, Pole 2007, Wangelin und Tuerk 2015). Die Absolutwerte der HR nähern sich zwar deskriptiv über die ganze Messung hinweg der Kontrollgruppe an, eine signifikante Veränderung lässt sich jedoch statistisch nicht bestätigen. Zudem deutet die Sensitivitätsanalyse insbesondere in Ruhe auf eine deutliche Störanfälligkeit der HR durch Betablocker hin, sodass die Unterschiede auch durch eine Konfundierung infolge der Medikation zustande kommen könnten. Der Graph des sBP verläuft bzgl. Baseline und Follow-up ähnlich, wobei zu beiden Messzeitpunkten die Reagibilität deutlich geringer als bei der Kontrollgruppe wirkt. Ähnliches gibt es zur BRS zu berichten, hier ist der Verlauf von Baseline und Follow-up sogar beinahe identisch. Für beide Parameter bestätigen die Recovery- und $\Delta$ R-Scores, dass eine Veränderung der Recovery zum Follow-up hin ausblieb und damit auch die Reagibilität als unverändert zu werten ist. Inwieweit die beiden Parameter eine geringe Änderungssensitivität besitzen oder schlicht kein Therapieeffekt vorlag, kann nicht bewertet werden, auch da es für BRS und sBP nur sehr wenige Langzeitstudien bei der PTBS gibt.

Baseline- und Follow-up-Graph des TPRI zeigen einen weitgehend parallelen Verlauf. Die $\Delta \mathrm{R}$-Scores weisen jedoch deskriptiv auf eine höhere Reagibilität im Follow-up hin. Wie es zu dieser Divergenz kommt ist unklar und kann anhand der Graphen nicht geklärt werden. Aufgrund des fehlenden graphischen Nachweises erscheinen die Ergebnisse aber zu lückenhaft, um dem TPRI eine höhere Reagibilität im Follow-up zuzusprechen. Im Gegensatz zu HR, sBP und BRS ist in der graphischen Analyse auch keine höhere Reagibilität der Kontrollgruppe beim TPRI erkennbar. Auffällig sind jedoch die deutlichen Unterschiede in den 
Absolutwerten aller drei Graphen des TPRI. Das Follow-up wies durchgehend die höchsten TPRI-Werte auf, während die Baseline-Messung höhere Werte als die Untersuchung der Kontrollgruppe ergab. Bestätigt wird diese Beobachtung durch signifikante Zeiteffekte des TPRI in allen durchgeführten ANOVAs. Es ist denkbar, dass die höheren TPRI-Werte im Follow-up auf einer Verschlechterung des Krankheitszustands der Patientinnen beruhen. So könnten die hohen TPRI-Werte aus einem allgemein erhöhten $\alpha$-adrenergen Aktivierungsniveau hervorgehen. Als Folge könnte eine Reflexbradykardie resultieren, die zu der im Vergleich zur Baseline-Messung deskriptiv verminderten Herzrate passen würde. Genauso ist der umgekehrte Mechanismus möglich: die niedrige HR, eventuell durch vermehrte Betablockereinnahme mitbedingt, könnte eine $\alpha$-adrenerge Gegenregulation zur Konstanthaltung des Blutdrucks hervorrufen. Der TPRI könnte jedoch auch durch andere Faktoren beeinflusst worden sein. So sind circadiane Schwankungen des TPRI bekannt (Diamant et al. 2002). Ein einheitlicher Messzeitpunkt konnte jedoch aus Organisationsgründen nicht für Baseline und Follow-up eingehalten werden. Andererseits gibt es Hinweise, dass hormonelle Veränderungen wie die Menopause die TPRI-Regulation maßgeblich beeinflussen. Während vor der Menopause eine $\beta$-adrenerge Regulation einen entscheidenden Faktor darzustellen scheint, steht nach der Menopause die $\alpha$-adrenerge Steuerung bei Blutdruck und TPRI im Vordergrund (Briant et al. 2016, Joyner et al. 2016). Da es durchaus auch ältere Teilnehmerinnen gab und zwischen Baseline und Follow-up drei Jahren vergingen, in denen z. B. Teilnehmerinnen von prä- zu postmenopausal gewechselt haben könnten, sind trotz stabilen Blutdrucks solche Prozesse nicht auszuschließen. Neben den Betablockern könnten zudem auch andere Medikamente auf den TPRI einwirken. Für den TPRI sind insbesondere vaskulär wirkende Medikamente wie Calciumantagonisten von Interesse. Der Anstieg des TPRI würde dabei eher für eine geringere Einnahme dieser vasodilatatorischen Medikamentengruppe sprechen. Dem widersprechend stieg die Anzahl an Patientinnen, die Calciumantagonisten einnahmen, von Baseline zu Follow-up von zwei auf drei an. Dabei wurde bei einer Patientin das Medikament nach der Baseline-Messung abgesetzt, während es bei einer anderen Patientin neu angeordnet wurde. Eine dritte Patientin, die zum Zeitpunkt Baseline Calciumantagonisten in ihrer Medikation hatte, war aus der Baseline-Messung ausgeschlossen worden und wurde daher für diesen Messzeitpunkt nicht gezählt. In jedem Fall sprechen diese Zahlen eher gegen Calciumantagonisten als Erklärung für die erhöhten TPRI-Werte.

Die Arbeitsgruppe von Vrana et al. (2009) entdeckte eine Assoziation von (versteckter) Feindseligkeit und dem Blutdruck bei PTBS-Probandinnen. In einer Studie mit 70 Probandinnen mit und 50 größtenteils traumaexponierten Probandinnen ohne PTBS wurde ein Ärgertest absolviert. Die PTBS-Probandinnen mit hohen Werten für Feindseligkeit zeigten dabei eine höhere HR während des Ärgertests und der Recovery sowie einen höheren diastolischen Blutdruck in Ruhe. Gleichzeitig konnte aber kein signifikanter Unterschied bei der Höhe des Blutdrucks in Ruhe zwischen Probanden mit und ohne PTBS gefunden werden. Der sBP zeigte zwar keine signifikante Assoziation zu dem Vorhandensein von Feindseligkeit bei der PTBS, die Daten deuten hier aber in eine ähnliche Richtung wie bei dem dBP 
(Vrana et al. 2009). Der TPRI wurde in der Studie nicht erhoben. Ein hoher TPRI führt jedoch i. d. R. auch zu einem höheren dBP. Es ist somit denkbar, dass sich die Eigenschaft „versteckte Feindseligkeit“ nicht nur in einem höheren dBP in Ruhe, sondern auch in einem höheren TPRI auswirkt. Die Teilnehmerinnen der vorliegenden Studie zeichneten sich durch einen Anstieg der Skala „Aggressivität/Feindseligkeit“ der SCL-90-R von im Mittel 5,2 zum Zeitpunkt Baseline auf 6,4 im Follow-up aus. Auch die Skala „Feindseligkeit“ des BSL verzeichnete eine Zunahme von im Mittel 4,9 in der Baseline-Messung auf 6,8 im Follow-up. Der während der ganzen Messung höhere TPRI im Follow-up könnte somit mit der Zunahme der Feindseligkeit bei den PTBS-Probandinnen im Zusammenhang stehen.

Der PEP-Graph des Follow-ups zeigt rein deskriptiv einen deutlich anderen Verlauf als der Baseline- oder der Kontrollgraph, sodass ein Vergleich der Reagibilität schwierig ist. Es überwiegt jedoch der Eindruck, dass sich der Verlauf der PEP eher von dem Kontrollgraph entfernt, sodass nicht von einer physiologischen Besserung auszugehen ist. Die Recoveryund $\Delta \mathrm{R}$-Scores deuten zudem nicht auf eine deutliche Änderung der Reagibilität hin. Aufgrund des doch sehr abweichenden Verlaufs der PEP im Follow-up, stellt sich die Frage nach der Verlässlichkeit dieses Parameters. Mehrere Studien haben die Retest-Reliabilität der PEP bezüglich arithmetischer Tests und in Ruhe bei gesunden Probanden evaluiert. Die PEP erreichte dabei zufriedenstellende Ergebnisse nach einer Woche (Burns et al. 1992), nach vier Wochen (Kasprowicz et al. 1990) und nach einem Jahr (Burleson et al. 2003). Kasprowicz et al. (1990) weisen insbesondere auf die im Vergleich zu den anderen Parametern hohe RetestReliabilität der PEP für die Rechen-Reactivity hin $(\mathrm{r}=0,78)$. Auch in einem ambulanten Setting präsentierte die PEP eine moderate Reproduzierbarkeit einer 24h-Messung nach zwei Monaten (Barnes et al. 2004). Lomb ermittelte eine Retest-Reliabilität für den Quotienten $\mathrm{PEP} /$ diastolischer Blutdruck von $\mathrm{r}=0,64$ bis $\mathrm{r}=0,75$ je nach Phase (Lomb 2011, S. 100). Insgesamt lassen sich die bisherigen Forschungsergebnisse somit mit einer mindestens modraten Retest-Reliabilität der PEP zusammenfassen. Die Korrelationen für Ruhe und Rechentest bewegten sich dabei bei den o.g. Studien in einem Bereich von $r=0,57$ bis $r=0,84$. Die PEP ist damit insgesamt nicht mehr, aber auch nicht weniger verlässlich als andere Parameter in der Psychophysiologie.

Zusammenfassend konnte keine verstärkte Reagibilität im Follow-up beobachtet werden. Da es Hinweise darauf gibt, dass eine höhere körperliche Fitness mit einer abgeschwächten Reaktivität auf mentale Stressoren einhergeht, lohnt zusätzlich ein Blick auf die sportliche Aktivität der Patientinnen (Forcier et al. 2006). Hier zeigte sich jedoch, dass es im Mittel keine wesentlichen Unterschiede in der wöchentlichen körperlichen Betätigung der Patientinnen zwischen Baseline und Follow-up gab. Somit ist ein Einfluss der körperlichen Fitness auf den Verlauf der kardiovaskulären Reagibilität der Patientinnen unwahrscheinlich. 


\subsection{Hypothese 4 bis 6: Wechselwirkungen zwischen Psyche, Physiologie und Therapie}

Die Hypothesen 4 bis 6 verbindet die Frage nach den Zusammenhängen zwischen Psyche, Physiologie und Therapie, sodass eine Testung des Datensatzes auf eventuelle Korrelationen zwischen diesen Dimensionen erfolgte. Dabei steht im Vordergrund der Versuch, mögliche Wechselwirkungen aufzudecken, ein Nachweis kann durch die kleine Kohorte und die multiple Testung nicht erbracht werden. Die Hypothese 4 konzentriert sich auf die Relation zwischen Verbesserungen im Stressempfinden und einer höheren stressphysiologischen Reagibilität im Follow-up. Sie konnte nicht bestätigt werden, denn es finden sich keine Korrelationen zwischen den Differenzen im Stressempfinden und den $\Delta \mathrm{R}$-Scores. Dies ist überraschend, würde man doch intuitiv erwarten, dass eine höhere Funktionalität des ANS das Stressempfinden senkt und umgekehrt. Das subjektive Stressempfinden scheint somit eine von der körperlichen Stressregulation unabhängige Größe darzustellen.

Für den in Hypothese 5 vermuteten Zusammenhang zwischen Verbesserungen in der Psychometrie und einem breiteren Reaktionsvermögen im Follow-up fanden sich angesichts der multiplen Testung wenige Korrelationen. Der $\Delta R_{T P R I}$ Rechnen korrelierte signifikant mit den Differenzen der SCL-90-R (GSI) und der BSL-Gesamtskala sowie hochsignifikant mit den Differenzen des BPI-Cut-20. Da der TPRI jedoch zur Phase Musik hin ansteigt, er-

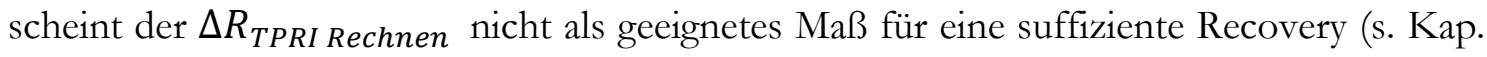
5.3.3). Die Aussagekraft dieser Korrelationen erscheint somit fraglich. Eine weitere hochsignifikante Korrelation ergab sich zwischen den Veränderungen des BDI und dem $\Delta R_{H R \text { Rechnen. }}$ Diese Korrelation ist interessanter, ist doch das BDI der einzige psychometrische Fragebogen, der sich zwischen Baseline und Follow-up signifikant verbessert hat. Für den $\Delta \mathrm{R}_{\text {HR Rechnen }}$ findet sich im Mittel keine Verbesserung im Sinne einer größeren Reagibilität im Follow-up. Dennoch zeigt diese (negative) Korrelation an, dass eine Verbesserung im BDI mit einer höheren Reagibilität im Follow-up einhergeht. Aus dem Boxplot zu dieser Korrelation (s. Abb. 18, S. 79) wird zudem ersichtlich, dass alle Patientinnen mit einem verschlechterten oder unveränderten BDI auch eine unveränderte oder gesunkene Reagibilität im Follow-up hatten. Es könnte somit sein, dass eine Besserung der depressiven Symptomatik mit einer verbesserten Reagibilität bezüglich des kognitiven Stressors Rechnen assoziiert ist. Für den Stressor Babyschreien findet sich dagegen keine Korrelation. Folglich könnte zumindest ein Teil der verminderten Reagibilität der PTBS durch die häufige depressive Komorbidität verursacht sein. Da sich die Korrelation jedoch in einer multiplen explorativen Testung ohne Bonferroni-Adjustierung fand, ist dieser Befund mit Vorsicht zu interpretieren. Er stellt nur einen ersten Hinweis dar, auf den weitere Forschung mit größeren Studienkohorten aufbauen kann. Grundsätzlich lässt sich aber resümieren, dass mit Ausnahme der soeben beschriebenen Korrelation (BDI und $\Delta R_{H R}$ Rechnen) kein Hinweis auf einen Zusammenhang zwischen Verbesserungen in der Psychometrie und einer Steigerung der Reagibilität gefunden werden konnte. Die Ergebnisse stehen im Widerspruch zu Boudewyns 
und Hyer (1990) sowie Rabe et al. (2006), die jeweils bei einer Therapieevaluation Korrelationen zwischen stärkeren Veränderungen in der Reaktivität auf traumaassoziierte Stressoren und psychologischen bzw. klinischen Verbesserungen beschrieben.

Die Hypothese 6 rückt die Therapie in den Mittelpunkt. Zeigen die Patientinnen, die sich länger in psychotherapeutischer/psychiatrischer stationärer Therapie befanden, auch deutlichere Verbesserungen im Stressempfinden, in der Psychometrie oder in der stressphysiologischen Reagibilität? Die Resultate dieser Studie sprechen nicht dafür. Die einzigen signifikanten Korrelationen bestehen zwischen der „Therapiedauer gesamt“ und der Differenz der IES-R-Skala Vermeidung, dem $\Delta R_{B R S \text { Babyschreien }}$ und dem $\Delta \mathrm{R}_{P E P \text { Rechnen. Gemessen }}$ an der Vielzahl der Tests sind die gefundenen Korrelationen so wenige und auf einem so niedrigen Signifikanzniveau ( $p<0,05$ ohne Bonferroni-Adjustierung), dass eher von Zufallssignifikanzen auszugehen ist. Es konnte folglich keine Assoziation zwischen der Therapiedauer und dem Stressempfinden, den psychometrischen Skalenwerte oder den $\Delta \mathrm{R}$-Scores festgestellt werden. Dieses Ergebnis gilt sowohl für die Therapiedauer aller stationären psychotherapeutischen oder psychiatrischen Behandlungen als auch für die Therapiedauer speziell auf der Station 9 des Asklepios Fachklinikums Göttingen. Stattdessen konnten jedoch mehrere positive Korrelationen zwischen dem Stressempfinden oder dem psychometrischen Befund zum Messzeitpunkt Baseline und der Therapiedauer gefunden werden - ein Zeichen, dass hier eine Assoziation bestehen könnte. Besonders schwer belastete Patientinnen nahmen besonders lange die Therapie in Anspruch. Dieser Zusammenhang erscheint einleuchtend. Die stärksten Korrelationen zeigten sich für das Stressempfinden in Ruhe 1 (Baseline) und die Therapiedauer auf Station 9 sowie für die IES-R-Skalen Intrusion und Vermeidung (Baseline) und die Therapiedauer aller psychotherapeutischen oder psychiatrischen Behandlungen. Ein hohes Stressempfinden in Ruhe 1 steht für ein im Alltag und somit auch allgemein erhöhtes Stressniveau - ein Kardinalsymptom der PTBS. Die IES-R ist ein Maß für die klassische PTBS-Symptomatik. Die PTBS-Symptomatik und der wahrgenommene Stress könnten folglich besonders belastend sein, wenn man davon ausgeht, dass sich ihr Ausmaß in der Therapiedauer widerspiegelt.

Eine mögliche Assoziation zwischen Therapiedauer und Stressempfinden bzw. dem psychischen Befinden während der Baseline-Messung stellt jedoch zumindest statistisch keine Erklärung dafür da, dass sich zwischen der Therapiedauer und den Veränderungen im Stressempfinden, in der Psychometrie und in der Stressphysiologie von Baseline zum Follow-up kaum signifikante Korrelationen finden. Dies ergibt sich aus der Tatsache, dass auch bei Kontrolle der Baseline-Werte in einer multivariaten Regression kein signifikanter Zusammenhang gefunden werden konnte. Die Hypothese 6 ließ sich somit nicht bestätigen. Ein Therapieeffekt ist jedoch weiterhin möglich, denn er muss nicht zwingend von der Therapiedauer abhängig sein. Im Gegenteil könnte eine lange Therapiedauer auch Zeichen einer schwierigeren Therapierbarkeit sein. Der Befund, dass schwerere Belastungen der Patientinnen mit einer längeren Therapiedauer korrelieren, untermauert diese These zusätzlich. 


\subsection{Psychophysiologische Evaluation der Therapiewirkung}

Die zentrale Frage dieser Follow-up-Studie ist, ob sich die Therapie in Veränderungen im Stressempfinden, in der Psychometrie oder in der Stressphysiologie widerspiegelt. Eine mögliche Therapiewirkung auf das kardiovaskuläre System steht hierbei besonders im Fokus - gibt es doch bislang nur wenige Studien, die sich dieser Fragestellung widmeten. Dabei ist eine Antwort von besonderer klinischer Bedeutung, schließlich geht die PTBS mit einem deutlich erhöhten kardiovaskulären Risiko einher (Edmondson et al. 2013).

Anhand der vorangegangenen Kapitel konnte keine stressphysiologische Veränderung durch die Therapie festgestellt werden. Die verminderte autonome Reagibilität der PTBS-Patientinnen präsentierte sich über die verschiedenen Messphasen und bei den meisten Parametern im Follow-up weitgehend unverändert zur Baseline-Messung. Dieser Befund soll nun anhand der uns bekannten Studien, die ebenfalls die kardiovaskuläre Stressreagibilität von PTBS-Patienten vor und nach einer Therapie erfassten, diskutiert werden (Boudewyns und Hyer 1990, Shalev et al. 1992, Blanchard et al. 2002, Karl et al. 2004, Lindauer et al. 2006, Rabe et al. 2006, Griffin et al. 2012, Wangelin und Tuerk 2015). All diese Studien legen einen Therapieeffekt in Form einer verringerten Reaktivität auf traumaassoziierte Stimuli nahe. Die in der Regel untersuchte kognitive Verhaltenstherapie scheint demnach einen Einfluss auf das ANS auszuüben. Die aktuelle Datenlage wirkt somit auf den ersten Blick eindeutig. Die vorliegende Studie widerspricht diesem Befund nicht - ein traumaassoziierter Stimulus wurde nicht betrachtet. Sie setzt jedoch den Fokus auf einen anderen Bereich der autonomen Dysregulation bei der PTBS. Wie in Kapitel 5.3.2.1 diskutiert, ist es denkbar, dass PTBSPatienten zwar auf traumaassoziierte Reize mit einer kardiovaskulären Hyperreaktivität (insbesondere der HR) reagieren, grundsätzlich aber, beispielsweise bei kognitiven und sozialen traumaunabhängigen Stressoren, die allgemeine Hyporeagibilität im Vordergrund steht. Es könnte sein, dass eine Hypoaktivität und Hyporeagibilität des Parasympathikus für beide Reaktionsmuster einen verbindenden Mechanismus darstellen. Die mangelnde vagale Regulation könnte sich z. B. in einer fehlenden vagalen Kontrolle und in einer fehlenden vagalen Disinhibition äußern. Warum es bei traumaassoziierten Stressoren trotz fehlender vagaler Disinhibition zu einer Hyperreaktivität von z. B. der HR kommt, ist unklar. Eventuell ist die Reaktivität des Sympathikus bei der PTBS stressor- bzw. traumaspezifisch und reagiert bei traumassoziierten Reizen über. Gleichzeitig scheint aber auch die mangelnde vagale Kontrolle eine Rolle zu spielen (Sack et al. 2004). In jedem Fall scheint es sich aber um eine traumaspezifische Stressreaktion zu handeln, die sich in ihrer autonomen Regulation von den Reaktionen auf andere Stressoren deutlich unterscheidet. Bei den traumaunabhängigen Stressoren, wie sie in der vorliegenden Studie eingesetzt wurden, scheint eher die Hyporeaktivität im Vordergrund zu stehen. Diese wird vermutlich durch die vagale Hyporeagibilität und die damit verbundene fehlende vagale Disinhibition bedingt. Durch das fehlende Absinken des Parasympathikus und seine weiterhin hemmende Wirkung auf den Sympathikus reagiert auch das sympathische System auf den Stress vermindert. Aber auch eine primäre sympathische 
Hyporeaktivität bei traumaunabhängigen Stressoren kann gegenwärtig nicht ausgeschlossen werden. Da die aktuelle Datenlage zu dem Zusammenspiel von Sympathikus und Parasympathikus bei unterschiedlichen Stresssituationen für die PTBS noch sehr lückenhaft ist, sind all diese Annahmen nicht ausreichend fundiert. Zudem sind die bisherigen Studien in vielen Punkten inkonsistent, es finden sich immer wieder widersprüchliche Befunde. So ist z. B. anzumerken, dass unter den traumaunabhängigen Stressoren die Schreckreaktionen wiederum oft zu einer Hyperreaktivität bei der PTBS führten. Außerdem deutet zwar die Mehrheit der uns bekannten Arbeiten zur vagalen Reaktion auf traumaassoziierte Reize auf eine vagale Hyporeaktivität der PTBS (zum Teil allerdings nur indirekt) hin (Cohen et al. 1998, Cohen et al. 2000, Sack et al. 2004, Hauschildt et al. 2011, Arditi-Babchuk et al. 2009), aber auch Gegensätzliches zu der vagalen Regulation während solcher traumabezogenen Stresssituationen wurde berichtet (Keary et al. 2009). Eine widerspruchslose Interpretation der aktuellen Datenlage ist daher nicht möglich und es ist an zukünftigen Studien die Inkonsistenzen aufzuklären. In jedem Fall muss bei einer Evaluation der Therapiewirkung jedoch beachtet werden, dass hinter traumaassoziierten und traumaunabhängigen Stressreaktionen unterschiedliche autonome Regulationsvorgänge stehen könnten. Ausgehend von der beobachteten allgemeinen Hyporeagibilität der PTBS-Probandinnen in der vorliegenden Studie ist eine Reaktivitätssteigerung als ein Therapieerfolg im Sinne einer Annäherung an gesunde Probanden zu verstehen. In den oben genannten Studien wurde dagegen eine Reaktivitätsminderung als Therapieerfolg gewertet, da sie sich in der Regel auf die traumaassoziierte Hyperreaktivität beriefen. Somit betrachtet die vorliegende Arbeit allein durch die ausgewählten Stressoren einen anderen Teil der autonomen Pathologien der PTBS.

Folglich werden für die Einordnung der Ergebnisse Studien benötigt, die ebenfalls einen traumaunabhängigen Stressor zur Therapieevaluation bei der PTBS nutzten. Bei der Auswahl der eingesetzten Stressoren zeigten sich die vorangegangenen Studien jedoch erstaunlich homogen. Ein Großteil verwendete skriptgesteuertes Erinnern an traumatische Erlebnisse als Stimulus. Selten erfolgte wie bei Griffin et al. (2012) und Karl et al. (2004) auch eine Aktivierung durch aufschreckende auditive Reize. Für den sonst in der Forschung beliebten Rechentest als kognitiven Stressor ist uns dagegen bislang nur eine ältere Studie von Blanchard et al. (1996) bekannt, die bei PTBS-Probanden die Reactivity auf Rechenstress auch in einem Follow-up evaluierte. Bei den 125 Probanden mit und ohne PTBS, die allesamt einen Verkehrsunfall in der Vorgeschichte erlebt hatten, konnte dabei eine globale Reduktion der Reaktivität (gemessen durch HR, sBP, dBP und EMG) nach einem Jahr festgestellt werden. Aber nur bei den 45 Teilnehmern mit PTBS erreichte die Abnahme der Reaktivität auch das Signifikanzniveau - für den Rechenstressor sowie für einen der traumaassoziierten Stressoren. Gleichzeitig verzeichneten etwa zwei Drittel dieser PTBS-Probanden auch eine klinische Besserung. In der Baseline-Messung hatten sie sich rein deskriptiv durch eine höhere HR-Reaktivität auf den traumaassoziierten Stressor ausgezeichnet, während sie sich beim Rechnen bzgl. der HR-Reaktion nur wenig von den anderen Teilnehmern unterschieden, in jedem Fall aber keine gesteigerte Reaktivität präsentierten. Da eine Therapie- 
evaluation nicht Ziel von Blanchard et al. war, ist nicht bekannt ob und wie die Probanden zwischen den beiden Messzeitpunkten therapiert wurden (Blanchard et al. 1996). Während die signifikante Reduktion der Reaktivität auf den traumaassoziierten Stress übereinstimmend mit den vorigen Studien einer klinischen Besserung entsprechen könnte, scheint die Reactivityabnahme beim Rechnen in Zusammenschau mit unseren Beobachtungen und der nicht gesteigerten Reactivity beim ersten Messzeitpunkt keine klinische Verbesserung, vielleicht sogar eine Verschlechterung, abzubilden. Auffällig ist zudem die allgemeine Reduktion der Reaktivität in dieser Studie, die auch die Probanden ohne PTBS aufwiesen. Der bei den Reaktionen auf traumabezogene Stressoren beobachtete Therapieeffekt in den oben genannten Studien und auch bei Blanchard et al. (1996) selbst, ist für den Rechenstress hier, wie auch in der vorliegenden Studie, nicht auszumachen.

Für die Diskrepanz zwischen den eher ernüchternden Ergebnissen der vorliegenden Studie und den bisher überwiegend vielversprechenden Therapieevaluationen in der Vergangenheit ist eine Vielzahl an möglichen Erklärungen denkbar. Zunächst ist hier natürlich ein eventuell fehlender Therapieerfolg zu nennen. Eine Therapiewirkung ist Voraussetzung, um überhaupt therapiebezogene Veränderungen in der Physiologie feststellen zu können. Das subjektive Stressempfinden spricht hierbei für eine erfolgreiche Therapie. Die Veränderungen im psychometrischen Befund sind mitunter inkonsistent und zeigen nicht gänzlich eine Besserung der Patientinnen an. Sie decken sich jedoch mit einer Vielzahl an Studien, die größtenteils für die gleichen Skalen und Symptomkomplexe eine Besserung bzw. keine Besserung verzeichneten und den eingesetzten Therapieformen insgesamt eine gute Wirkung bescheinigten (s. Kap. 5.2). Dabei gilt es zu beachten, dass in dieser Studie die chronifizierte komplexe PTBS nach Mehrfachtraumatisierungen in der Kindheit betrachtet wird, sodass in Anbetracht der schwierigen Therapierbarkeit bereits geringe Veränderungen einen Therapieerfolg darstellen (s. hierzu auch S. 113). Abzugrenzen davon ist die „klassische“ PTBS nach einem Typ 1-Trauma, die in der Regel mit einem deutlich besseren Therapieoutcome einhergeht. Die Verbesserungen der PTBS-Patientinnen in der vorliegenden Studie scheinen sich folglich im Rahmen der für dieses Krankheitsbild möglichen oder zumindest typischen Veränderungen zu befinden - insbesondere wenn man bedenkt, dass das Follow-up mit drei Jahren Abstand stattfand.

Die Therapie scheint sich somit - zumindest geringfügig - positiv auf Psyche und Stressempfinden auszuwirken. Für die Physiologie sind dagegen keinerlei Effekte auszumachen. Ein Grund hierfür könnte sein, dass die allgemeine autonome Hyporeagibilität der PTBS schwieriger als die traumaspezifische Hyperreaktivität durch Therapien zu beeinflussen ist. Es ist schlüssig, dass die Hyperreaktivität auf traumaassoziierte Reize ein greifbarer Ansatzpunkt für die Therapien ist. Die Traumaexposition ist Teil vieler etablierter PTBS-Therapieregime. Habituation ist bei der prolonged-exposure-Therapie z. B. ein Therapieziel (Gallagher und Resick 2012). Es ist nicht überraschend, dass eine so gezielte Therapie der traumaassoziierten Stressoren sich auch im Follow-up nach der Therapie in Form einer verringerten Reaktivität auf ebenjene Stimuli niederschlägt. Hiermit übereinstimmend verringerte sich die Reaktivität 
bei Shalev et al. (1992) nur bei traumatischen Erinnerungen, die gezielt mit systematischer Desensibilisierung therapiert wurden. Die Antwort auf andere, nicht therapierte traumatische Stimuli blieb trotz klinisch erfolgreicher Behandlung pathologisch (Shalev et al. 1992).

Es fällt zudem auf, dass sich die bisherige Forschung in Bezug auf die analysierten Parametern sehr ähnelt: Alle oben aufgeführten Studien haben entweder HR, EMG und/oder Hautleitfähigkeit als Maß für die Psychophysiologie verwendet. Eine Ausnahme sind Lindauer et al. (2016), die neben der HR auch sBP und dBP erhoben. Dabei reduzierte eine Psychotherapie signifikant die Mittelwerte des dBP in Ruhe und während der Recovery sowie des sBP in Ruhe, wie eine Aufteilung der PTBS-Probanden in Therapie und Warteliste zumindest nahelegte (Lindauer et al. 2006). Die überwiegende Verwendung der gleichen drei Parameter impliziert jedoch auch, dass bislang kaum multidimensionale Studien zu der Thematik existieren. Dies verkompliziert die Einordnung der Ergebnisse der vorliegenden Studie. Unseres Wissens wurden nur bei einer Studie zur Evaluation klassischer PTBS-Therapieverfahren vagale Parameter erhoben. Nishith et al. (2003) fanden bei fünf PTBS-Probandinnen nach einer kognitiven Verhaltenstherapie eine Verringerung der LF/HF-Ratio während des REMSchlafes, die sich bei einer Therapieabbrecherin nicht zeigte. Dies könnte vorsichtig als Hinweis auf eine Wirkung der kognitiven Verhaltenstherapie auf das vagale System gewertet werden. Allerdings stellt die Messung während des REM-Schlafes eine von einem Stresstest doch sehr abweichende Situation dar, sodass kein direkter Rückschluss auf die vagale Hyporeagibilität möglich ist. Zudem ist die kleine Fallzahl bei der Interpretation zu beachten.

Inwieweit ein Einfluss der etablierten Therapien auf die vagale Hyporeagibilität besteht, bleibt somit weitgehend ungeklärt. Zwar ist die häufig erhobene HR ebenfalls maßgeblich parasympathisch beeinflusst. Dennoch gibt sie keine eindeutige Auskunft darüber, ob es auch direkt zu einer Veränderung der vagalen Regulation kommt. Es ist möglich, dass die in den oben genannten Studien beschriebene Therapiewirkung auf eine überschießende Sympathikusreaktion beschränkt war, die vagale Hyporeagibilität jedoch unberührt von der Therapie blieb. Dies ist schlüssig, wenn die Therapiewirkung tatsächlich durch eine Habituation im Zuge der Traumaexposition zustande kam. Eine Habituation erscheint als eine geeignete Therapieform für eine Hyperreaktivität, eine Hyporeagibilität erreicht sie jedoch vermutlich nicht. Die Therapie der vorliegenden Studie könnte somit die gleiche Wirkung auf das ANS der PTBS gehabt haben wie die Therapien in den oben beschriebenen Studien. Da jedoch kein traumaassoziierter Stressor und damit auch nicht die kardiovaskuläre Hyperreaktivität erhoben wurden, könnte dieser Therapieeffekt verborgen geblieben sein. Gleichzeitig ist die vorliegende Studie unseres Wissens die erste, die gezielt die Therapiewirkung auf die vagale Stressregulation prüft. Da hier keine Veränderung der vagalen und allgemeinen Hyporeagibilität festgestellt werden konnte, ist eine allgemeine Therapieresistenz des vagalen Systems bezüglich der etablierten Therapieformen nicht auszuschließen. Würde jedoch mit den aktuellen Therapieformen nur die traumaassoziierte Hyperreaktivität und nicht die vagale Hyporeagibilität erreicht, bliebe ein Teil der autonomen Dysregulation der PTBS von der 
Therapie unberührt. Es ist nicht unwahrscheinlich, dass gerade die Hyporeagibilität, die sich in Ruhe und bei kognitivem und emotionalem Stress zeigt und damit im Alltag vermutlich im Vordergrund steht, erheblich zum kardiovaskulären Risiko der PTBS beitragen könnte (s. Kap. 1.4.2). Auch ein deutlicher Einfluss auf die Lebensqualität ist naheliegend. Betrachtet man somit nur die Reaktion auf traumaassoziierte Stressoren könnte ein erheblicher Teil der autonomen Pathologien verborgen bleiben.

Da ansonsten die Datenlage zu der PTBS bislang wenig Vergleiche ermöglicht, lohnt sich ein Blick auf ähnlich aufgebaute Studien - auch wenn diese sich nicht der PTBS widmeten. Während der Task Force ${ }^{\circledR}$ Monitor schon häufig in der psychophysiologischen Stresstestung zum Einsatz kam, wurde er nur selten zur Evaluierung einer Therapiewirkung auf das ANS eingesetzt. Lomb et al. (2015) bilden eine Ausnahme und verwendeten ebenjenes Monitoringsystem, als sie eine psychosomatische Patientenkohorte vor und nach einer vier- bis sechswöchigen akutpsychosomatischen stationären Therapie mithilfe von Taktatmung, Rechentest und Ärgertest untersuchten (s. Kap. 5.1). Es erfolgte eine Betrachtung der Stressphysiologie unter Berücksichtigung der verschiedenen Achsen des ANS inklusive des Vagus. Während die Kohorte der vorliegenden Studie jedoch einheitlich aus Patientinnen mit komplexen Traumafolgestörungen bestand, rekrutierten Lomb et al. eine gemischte psychosomatische Patientengruppe mit unterschiedlichen Diagnosen. Außerdem fand das Follow-up bei Lomb et al. (2015) direkt im Anschluss an die Therapie nach vier bis sechs Wochen statt, während in der vorliegenden Studie drei Jahre zwischen den Messungen lagen. Lomb et al. konnten in ihrer Studie für keinen physiologischen Parameter eine signifikante Veränderung zeigen - weder in Ruhe noch unter Stress. Gleichzeitig verbesserten sich das Stressempfinden sowie der psychometrische Befund jedoch relevant. Damit kommen Lomb et al. zu sehr ähnlichen Ergebnissen wie die vorliegende Studie. Dies ist v. a. interessant, da Lomb et al. ebenfalls eine Hyporeagibilität der Patienten gegenüber der Kontrollgruppe beschreiben. Trotz deutlicher Verbesserungen in der Psychometrie blieb diese verringerte Stressreagibilität jedoch von der Therapie weitgehend unberührt (Lomb et al. 2015, Lomb 2011). Dies kann als weiterer Hinweis für einen fehlenden Therapieeffekt auf die Hyporeagibilität gewertet werden, auch wenn er sich bei der Betrachtung einer anderen Patientenkohorte ergab.

Darüber hinaus sind weitere Gründe denkbar, warum die vorliegende Arbeit im Gegensatz $\mathrm{zu}$ anderen Studien keine Therapiewirkung auf das ANS zeigt. So ist uns bislang keine weitere Studie bekannt, die eine physiologische Therapieevaluation bei den komplexen Traumafolgestörungen durchführte. Die meisten Studien konzentrierten sich auf Kohorten mit Kriegsveteranen oder Verkehrsunfallopfer, während die an sich so häufigen Traumatisierungen von Frauen durch sexuelle und körperliche Gewalt bislang selten Gegenstand der Forschung zur Therapiewirkung bei der PTBS waren. Tatsächlich sind uns nur zwei Studien zu dieser Thematik bekannt. Griffin et al. (2012) zeichneten die Reaktivität auf Schreckreize von 74 weiblichen Opfern von körperlicher oder sexueller Gewalt in Form von HR, Hautleitfähigkeit und EMG vor und nach einer sechswöchigen kognitiven Verhaltenstherapie auf. Responder, die nach der Therapie nicht mehr die PTBS-Diagnose erfüllten, zeichneten sich durch eine 
signifikant geringere Reaktivität bei allen drei Parametern im Vergleich zu ihren Werten vor der Therapie und bei HR und EMG im Vergleich zu Nonrespondern aus (Griffin et al. 2012). In einer kleinen, oben bereits erwähnten Studie entdeckten Nishith et al. (2003) anhand von HRV-Indices eine Abnahme der sympathischen Dominanz während des REM-Schlafes bei fünf Vergewaltigungsopfern, die erfolgreich zuvor eine kognitive Verhaltenstherapie absolviert hatten. Eine sechste Patientin, die die Therapie abbrach, verbuchte dagegen bei gleichzeitig weiterhin bestehender Symptomatik einen Anstieg der Sympathikusaktivität während des REM-Schlafes (Nishith et al. 2003). Da das Krankheitsbild der komplexen Traumafolgestörungen häufig bei Opfern von sexueller und physischer Gewalt ist, ist es nicht unwahrscheinlich, dass unter den von Griffin et al. (2012) und Nishith et al. (2003) untersuchten Probandinnen auch Frauen mit einer komplexen Traumafolgestörung waren. Aber ohne eingehendere Diagnostik zu diesem Krankheitsbild ist im Nachhinein nicht nachvollziehbar, inwiefern die beiden Studien diese Patientengruppe abbilden. Wie bereits beschrieben untersuchten Nishith et al. (2003) zudem mit der autonomen Regulation bei REM-Schlaf eine doch sehr andere Fragestellung als die vorliegende Studie. Griffin et al. (2012) dagegen bieten eher eine Vergleichsmöglichkeit. Beide Studien legen im Gegensatz zu der vorliegenden Studie einen messbaren Therapieeffekt nahe. Dennoch ist es einleuchtend, dass eine Therapiewirkung auf das ANS bei einer reinen Kohorte mit komplexen Traumafolgestörungen nur sehr schwierig erreicht werden kann. Diese Patientengruppe gilt im Vergleich zur „klassischen" PTBS als schwierig therapierbar und ist oft durch eine besondere Krankheitsschwere gekennzeichnet. Durch die frühe Traumatisierung kommt es zu persistierenden epigenetischen Abweichungen. Die Stresssysteme sind grundlegend verändert und zum Teil finden sich sogar hirnmorphologische Auswirkungen (s. Kap. 1.3). Mutmaßlich ist es sehr schwierig, diese tiefgreifenden biologischen Langzeitfolgen mit einer Therapie zu beeinflussen. Wahrscheinlich muss sich das klinische Bild erheblich bessern, um auch messbare Verbesserungen in der Physiologie zu erreichen. Zudem gehen die komplexen Traumafolgestörungen mit deutlich mehr Komorbiditäten einher als die „klassische“ PTBS, die sich wiederum zusätzlich auf die Stressregulation auswirken können. Der Nachweis einer Therapiewirkung auf die autonome Dysfunktion der „klassischen“ PTBS, wie sie häufig bei Opfern von Verkehrsunfällen oder Kriegsveteranen zu finden ist, kann folglich nicht zwangsläufig auf eine Kohorte mit komplexen Traumafolgestörungen übertragen werden. Die Diskrepanz zwischen den berichteten und der vorliegenden Studie könnte somit auf den zum Teil sehr unterschiedlichen Krankheitsbildern der untersuchten Probanden beruhen.

Gerade Komorbiditäten wie Depressionen könnten die Therapieevaluation stören. Interessanterweise scheint eine Besserung der depressiven Symptomatik mit einer höheren Reagibilität für das Rechnen im Follow-up einherzugehen, wobei der Befund aufgrund der multiplen Testung mit Vorsicht interpretiert werden muss (s. Kap. 5.4). Trotz signifikant niedrigerem BDI im Follow-up ist jedoch die Reagibilität während des Rechnens im Mittel unverändert. Das Ausmaß der durch die verbesserte depressive Symptomatik angestiegenen Reagibilität kann daher nicht sehr groß sein. Die depressive Symptomatik scheint folglich auch keine 
PTBS-bezogene Besserung der Reagibilität verdeckt zu haben. Vielmehr ist es möglich, dass eine PTBS-bezogene Verschlechterung der Rechenreagibilität mancher Patientinnen verborgen blieb, da die Reduktion der depressiven Symptomatik gleichzeitig zu einer höheren Reagibilität führte. Es stellt sich zudem die Frage, welchen Anteil die depressive Symptomatik allgemein an der Genese der Hyporeagibilität bei der PTBS haben könnte. Bemerkenswert ist, dass auch für die Depression die Datenlage inkonsistent ist und ebenfalls sowohl eine Hyperreaktivität (Light et al. 1998, Kibler und Ma 2004) als auch eine Hyporeaktivität (York et al. 2007, Salomon et al. 2009, Phillips et al. 2011) auf Stressoren berichtet wird. Hu et al. (2016) sahen wiederum auch für die Depression und Angsterkrankungen eine stressorabhängige Reaktivität, nachdem sie eine Hyporeaktivität bei einem kognitiven Stressor und eine Hyperreaktivität bei einem psychiatrischen Interview (mit emotionalen Stress bezogen auf die Symptomatik der Depression/Angsterkrankungen) beobachtet hatten. Sie vermuten auch hier eine veränderte vagale Regulation als entscheidenden Pathomechanismus, wobei jedoch keine allgemeine Hyporeaktivität des Parasympathikus bestand, sondern auch eine vagale Hyperreaktivität bei dem psychiatrischen Interview festgestellt werden konnte (Hu et al. 2016). Damit übereinstimmend war eine verminderte RSA-Reaktivität bei einer aktuellen Depression, nicht aber bei einer remittierten Depression zu finden (Bylsma et al. 2014). Es ist somit durchaus denkbar, dass zumindest ein Teil der in dieser Studie beobachteten Hyporeagibilität durch die depressive Symptomatik mitbedingt sein könnte. Hughes et al. (2007) verglichen die vagale Regulation von Probanden mit einer PTBS (ohne Depression), mit einer PTBS und einer komorbiden Depression oder mit einer Depression (ohne PTBS) sowie von einer gesunden Kontrollgruppe miteinander. Zwar zeigten lediglich die Gruppen „nur PTBS“ und „PTBS mit Depression“ eine signifikant niedrigere BRS in Ruhe. Aber gemessen an der Effektgröße und in Anbetracht der kleineren Stichprobe und einer größeren Variabilität der Werte ist für die Gruppe „nur Depression“ trotz fehlender Signifikanz ebenfalls eine niedrigere BRS in Ruhe anzunehmen. Während eines Ärgertests war der Abfall der BRS bei den Patientengruppen (PTBS, PTBS und Depression, Depression in einer Gruppe zusammengefasst) signifikant geringer als bei der Kontrollgruppe (Hughes et al. 2007). Das vagale System scheint hiernach sowohl bei der PTBS als auch bei der Depression verändert zu sein. Es könnte sein, dass es sich bei der vagalen Hypoaktivität und -reagibilität nicht um ein spezifisches Symptom der PTBS handelt. Andererseits sind die Abweichungen in der vagalen Regulation auch bei einer PTBS ohne eine komorbide Depression nachzuweisen. Weil gerade für die Gruppe „,nur Depression“ aufgrund mangelnder Signifikanz nicht eindeutig geklärt ist, ob diese tatsächlich mit einer verminderten vagalen Ruheaktivierung einhergeht, ist eine eindeutige Interpretation nicht möglich. Weitere Forschung ist zur Rolle der Depression bei der autonomen Dysfunktion der PTBS notwendig.

Dafür könnte die dissoziative Symptomatik, die eventuell ebenfalls mit einer Hyporeagibilität einhergeht (s. Kap. 5.3.2.2), eine Therapiewirkung auf die PTBS-Symptomatik verschleiert haben. Schließlich hat sich die dissoziative Symptomatik zwischen den beiden Messzeitpunkten nicht gebessert. Auch hier wäre aber Voraussetzung für einen solchen Effekt, dass die 
Dissoziation einen erheblichen Teil der Hyporeagibilität mitbedingt, gerade da sie nicht angestiegen ist. Zudem ist die Dissoziation Teil des Symptomkomplexes der komplexen Traumafolgestörung und eine Verringerung der dissoziativen Symptomatik kann zu den Therapiezielen gerechnet werden. Konkrete Hinweise auf einen entscheidenden Einfluss der Dissoziation auf die Stressreagibilität, sind in dieser Studie allerdings nicht zu finden, v. a. da die $\Delta$ R-Scores auch keine Korrelation zu den Veränderungen im FDS ergaben. Auch hier ist es an zukünftigen Studien den Anteil der Dissoziation an der Hyporeagibilität zu differenzieren.

Eine Verzerrung der Ergebnisse durch die Medikation ist ebenfalls nicht auszuschließen. Für die Betablockermedikation zeigte sich zwar anhand der Sensitivitätsanalyse nur in Ruhe ein klarer Einfluss. Dennoch wurde von den Patientinnen eine Vielzahl an Medikamenten eingenommen, die nicht allesamt evaluiert werden können. Andere Autoren, die den Einschluss von Patienten mit Medikation betrachteten, konnten keine Hinweise auf gravierende Auswirkungen feststellen (Pole 2007, Hughes et al. 2007, Bornschein 2014). Die Frage nach dem Einfluss der Medikation kann jedoch dennoch nicht abschließend geklärt werden.

Zuletzt spielt vermutlich der zeitliche Abstand des Follow-ups eine bedeutende Rolle. Die oben beschriebenen Studien erhoben in der Regel ihr Follow-up im direkten Anschluss an die Therapie. Ebenso wie psychometrische könnten auch physiologische Effekte mit der Dauer des Abstands von der Therapie verblassen. Es könnte sein, dass die in den anderen Studien beschriebenen Therapieerfolge auf das ANS mit einem Abstand von drei Jahren ebenfalls nicht mehr zu erheben gewesen wären. Ziel ist es jedoch langfristige Verbesserungen in der autonomen Regulation der PTBS-Patientinnen zu bewirken. Findet sich nach drei Jahren kein bleibender Effekt, muss eine Optimierung der Therapie versucht werden.

Insgesamt ist nicht eindeutig zu klären, warum sich in dieser Studie im Gegensatz zu vorausgegangener Forschung keine Therapiewirkung auf das ANS der PTBS abzeichnet. Die Erklärungsansätze reichen von einer Therapierefraktärität der (vagalen) Hyporeagibilität über eine unzureichende Ausrichtung des Therapiekonzeptes auf die autonome Dysregulation bis zu einer Konfundierung durch Komorbiditäten oder Medikation. Auch könnte eine Therapiewirkung bei Patientinnen mit komplexen Traumafolgestörungen besonders schwierig zu erreichen sein. Die verschiedenen Erklärungsansätze widersprechen sich dabei nicht zwingend. Eine überzeugende Erklärung für die Inkonsistenzen ist jedoch, dass bisherige Therapieevaluationen lediglich die traumaassoziierte Hyperreaktivität betrachteten und die allgemeine Hyporeaktivität außer Acht ließen. Gleichzeitig wurde das vagale System unzureichend berücksichtigt. Die vorliegende Studie deutet in jedem Fall darauf hin, dass die etablierten Therapien keine ausreichende Wirkung auf die vagale und allgemeine Hyporeagibilität besitzen. In diesem Fall gilt es, zusätzliche Therapiekonzepte zu entwickeln, die speziell das ANS erreichen. So könnte Biofeedback eine effektive Methode sein, die autonome Dysfunktion bei der PTBS gezielt zu therapieren. Bei Tan et al. (2011) zeigten Kriegsveteranen, die zusätzlich zu einer nicht genauer eingegrenzten Routinetherapie (treatment as usual) eine spezifische HRV-Biofeedback-Therapie erhielten, nach 8 Wochen weniger PTBS-assoziierte Symptome 
sowie einen Anstieg der HRV in Ruhe. Bei Probanden, die lediglich die Routinetherapie absolvierten, konnten für HRV und PTBS-Symptomatik keine signifikanten Veränderungen nachgewiesen werden. Vor der Therapie hatten sich die insgesamt 20 Kriegsveteranen gegenüber einer Kontrollgruppe durch eine signifikant niedrigere HRV in Ruhe ausgezeichnet (Tan et al. 2011). Bemerkenswert ist, dass in dieser Studie nicht nur effektiv die verringerte HRV therapiert werden konnte, sondern die Hinzunahme der Biofeedback-Therapie gleichzeitig zu einer stärkeren Besserung der PTBS-Symptomatik führte. Dies ist ein Hinweis, dass die PTBS-typische Symptomatik durch die autonome Dysregulation mitbedingt oder zumindest verstärkt sein könnte und eine Therapie des ANS somit zu einer Verbesserung des Gesamtzustands führen könnte. Auch Zucker et al. (2009) schrieben der Biofeedback-Therapie im Vergleich zu der progressiven Muskelrelaxation eine Senkung der depressiven Symptomlast sowie eine Steigerung der HRV bei Patienten mit PTBS-Symptomatik zu, wobei die Probanden auch durch Substanzmissbrauch in der Vorgeschichte gekennzeichnet waren. Zu beachten ist, dass laut Zucker et al. (2009) eine Reduktion der Ruheatmung bei der Biofeedback-Gruppe einen Teil des HRV-Anstiegs vermittelt haben könnte. Die PTBS-Symptomatik besserte sich bei beiden Therapieformen, wobei der HRV-Anstieg mit der Besserung der PTBS-Symptomatik korrelierte (Zucker et al. 2009). Auch wenn sowohl die progressive Muskelrelaxation als auch das Biofeedback auf die Physiologie der Stressregulation abzielen, zeigen die beiden Therapien doch unterschiedliche Effekte. Zukünftige Therapiekonzepte müssen daher spezifisch auf die PTBS abgestimmt und umfassend evaluiert werden. Zudem weist die Studie von Zucker et al. (2009) auch auf die weiterhin ungeklärte Rolle der komorbiden Depressivität bei der Stressregulation der PTBS hin, da sie einen großen Effekt auf die autonome Reagibilität zu haben scheint. Auch autogenes Training könnte einen Einfluss auf das ANS der PTBS haben. Mitani et al. (2006) fanden bei Feuerwehrmännern, die nach der IES$\mathrm{R}$ in eine PTBS-Gruppe und in eine Kontrollgruppe eingeordnet wurden, anhand von HRIndizes einen Anstieg der Parasympathikus- und einen Abfall der Sympathikusaktivität nach einem autogenen Training. Vor dem Training hatte sich die PTBS-Gruppe gegenüber der Kontrollgruppe durch eine pathologische sympathovagale Balance mit einem Übergewicht des Sympathikus ausgezeichnet. Da das autonome Training auf beide Gruppen die gleiche Wirkung zeigte, blieb auch nach der Therapie ein Unterschied bestehen, auch wenn der Therapieeffekt bei der PTBS-Gruppe deskriptiv größer war. Interessant ist zudem, dass bei einer alleinigen Therapie mit autogenem Training auch die IES-R bei der PTBS-Gruppe signifikant sank (Mitani et al. 2006). Des Weiteren gibt es erste Hinweise, dass auch Yoga einen Einfluss auf Symptomatik und HRV bei der PTBS besitzen könnte (Van der Kolk 2006). Entspannungsverfahren wie autogenes Training oder Yoga könnten somit eine Reduktion der Hyperarousal-Symptomatik bewirken und so einen positiven Einfluss auf das ANS haben. Es gibt folglich mehrere vielversprechende Methoden, die als Ergänzung zu den aktuellen Therapieregimen eingesetzt werden könnten. Zukünftig gilt es die verschiedenen Therapieansätze zu erforschen und mit gezielter Therapie die Wirkung auf das ANS zu verbessern. 


\section{Neue Forschungsansätze und Limitationen}

Durch neue Ansätze betritt die vorliegende Studie in mehreren Gebieten Neuland. Gleichzeitig sind einer insofern eher explorativen Studie von Natur aus Grenzen gesetzt, die nicht zuletzt die Umsetzbarkeit betreffen. Auf beide Aspekte soll im Folgenden eingegangen werden. Zunächst ist diese Studie unseres Wissens die erste, die eine psychophysiologische Therapieevaluation explizit bei Frauen mit komplexen Traumafolgestörungen vornimmt. Allgemein ist die Forschung zur Stressphysiologie von Frauen mit PTBS bislang unzureichend. Eine isolierte Betrachtung der komplexen Traumafolgestörungen fehlte völlig und ist aufgrund der besonderen Krankheitsschwere und der Häufigkeit dieses Krankheitsbilds umso wichtiger. Die differenzierte Betrachtung des ANS ist eine weitere Stärke der Studie: Sowohl sympathische als auch parasympathische Parameter wurden berücksichtigt. Lange wurde der Vagus in der stressphysiologischen Forschung zur PTBS, besonders in Bezug auf Therapieevaluationen, vernachlässigt. Die zunehmende Erkenntnis, dass der vagalen Regulation eine wichtige Rolle in der autonomen Dysregulation der PTBS zukommt, macht eine Berücksichtigung des Vagus aber unabdingbar. Die vorliegende Studie unterscheidet sich darüber hinaus von anderen Studien, indem sie mit den Stressoren Rechnen und Babyschreien alltägliche, traumaunabhängige Stresssituationen zur Therapieevaluation nutzte und so der in solchen Stressphasen beobachteten Hyporeagibilität der PTBS Beachtung schenkt. Durch die Kombination von differenzierter Erfassung der Stressreagibilität und Berücksichtigung traumaunabhängiger Stressoren konnte erstmals die Therapiewirkung sowohl auf die vagale als auch auf die allgemeine autonome Hyporeagibilität untersucht werden.

Eine weitere Neuerung ist die Betrachtung des Faktors Reagibilität. Bislang wurde in der Regel nur die Reaktivität auf einzelne Stressoren in Studien zur PTBS berücksichtigt. Da die PTBS-Probandinnen in der Baseline-Messung durch eine globale Hyporeagibilität auffielen, wurde mit den Recovery- und $\Delta \mathrm{R}$-Scores ein Maß der maximalen Reagibilität konzipiert. So sollte die Fähigkeit zur Stressregulation auch bezüglich der Recovery abgebildet und Zusammenhänge zwischen Reagibilität und Stressempfinden bzw. psychischer Belastung aufgedeckt werden. Da sich die Reagibilität von Baseline zu Follow-up weitgehend unverändert präsentierte, war eine Evaluation dieses Konzepts schwierig. Somit ist es an zukünftigen Studien, die Aussagekraft dieses Ansatzes zu bewerten. Die gleichzeitige Erfassung von Stressempfinden, Psychometrie und kardiovaskulärer Stressphysiologie offenbarte die Unabhängigkeit dieser drei Dimensionen. So erlaubte z. B. ein verbessertes Stressempfinden keine Rückschlüsse auf die Funktionalität der physiologischen Stressregulation. Außerdem zeigte sich u. a. beim Babyschreien eine Diskrepanz zwischen Stresserleben und Stressreaktion des Körpers: Das hohe subjektive Stressempfinden unterschied sich deutlich von der geringen physiologischen Reaktivität der Probandinnen. Hieraus ergibt sich für zukünftige Studien die Notwendigkeit zur Aufzeichnung aller drei Dimensionen. 
Mit dem Follow-up konnte durch Reproduktion der Baseline-Ergebnisse der dort erzielte Erkenntnisgewinn gefestigt und der Stressor Babyschreien reevaluiert werden. Da das Babyschreien grundsätzlich andere Stressregulationsprozesse als die etablierten Stressoren auslöste, konnte die Hypothese einer Generalisierbarkeit der vagalen Hyporeagibilität der PTBS auf ganz unterschiedliche Stresssituationen weiter gestützt werden. Auch konnte durch das Follow-up die zeitliche Stabilität der autonomen Dysbalance bei der PTBS aufgezeigt werden. Neben den Stressphasen wurde auch der bislang wenig erforschten Recovery-Phase Beachtung geschenkt. Hierbei wurde mit der Analyse einer eher „mittelfristigen“ Recovery mit etwas zeitlichen Abstand zu den Stressphasen eine neue Betrachtungsweise gewählt.

Neben diesen Stärken hat die Studie aber auch einige Limitationen. Auch wenn versucht wurde, beide Messungen möglichst identisch zu gestalten, waren einige Unterschiede unvermeidbar. Der Anreiseweg war im Follow-up bedeutend weiter als bei der Baseline-Messung und damit möglicherweise mit einem höheren Stress verbunden. Gleichzeitig befand sich ein Großteil der Patientinnen bei der Baseline-Untersuchung in der Diagnostikphase der Station 9 des Asklepios Fachklinikums Göttingen, welche eine besondere Situation für die Patientinnen darstellte. Im Follow-up reiste der überwiegende Anteil dagegen von zu Hause an. Die Baseline- und die Follow-up-Messung wurden jeweils von einer anderen Doktorandin vorgenommen. Zudem gab es zwischen den beiden Messungen einen Wechsel des Untersuchungsraums. Auch wenn die Untersuchungen genau gleich abliefen und die beiden Räume im Grunde sehr ähnlich gestaltet waren, ist ein Einfluss nicht auszuschließen. Zuletzt ist auch eine geringe Zeitdifferenz in der Durchführung zu nennen: Die Phase Ruhe 3 dauerte in der Follow-up-Messung fünf Minuten, bei der Baseline-Messung sechs Minuten.

Von den 50 angeschriebenen Baseline-Probandinnen nahmen nur 26 an dem Follow-up teil. In Kapitel 4.2.2 konnte kein Unterschied zwischen den „Nur Baseline“- und den Follow-upTeilnehmerinnen gefunden werden. Eine Teilnehmerrate von knapp über $50 \%$ mag niedrig erscheinen. In Anbetracht einer Patientenkohorte mit komplexen Traumafolgestörungen sind $50 \%$ jedoch durchaus gut - sind solche Patientinnen doch durch spezifische Charakteristika wie das häufige Vorliegen einer BPS und einer damit verbundenen Unbeständigkeit gekennzeichnet. Zudem war es durchaus bemerkenswert, dass einige der Patientinnen eine sehr weite und für sie belastende Anreise auf sich nahmen, um am Follow-up teilzunehmen. Dennoch handelt es sich um eine deutlich kleinere Stichprobe als die Ursprungsstichprobe. Dies wirkt sich auf die statistische Power und die Möglichkeit einer Subgruppenbildung aus. Aufgrund des Studiendesigns einer Follow-up-Studie war eine Rekrutierung zusätzlicher Probandinnen nicht möglich. Im Vergleich zu ähnlichen Studien in diesem Forschungsgebiet sowie in Anbetracht des dreijährigen Abstands des Follow-ups ist eine Anzahl von 26 Probandinnen jedoch durchaus als eine eher große Kohorte zu werten. Aufgrund der geringeren statistischen Power wurden einige Analysen lediglich explorativ durchgeführt. Ein statistischer Nachweis wäre wünschenswert gewesen, ist aber bei einer so kleinen Kohorte in Anbetracht der multiplen Testung unwahrscheinlich. Durch die Ermittlung geringerer, mit einer Bonferroni-Adjustierung nicht signifikanter Assoziationen konnten Fragestellungen für 
zukünftige Studien ausgelotet werden. Ein Risiko stellen hierbei falsch-positive Befunde dar. Tatsächlich konnten bei der multiplen Testung mit wenigen Ausnahmen keine Korrelationen zwischen Reagibilität, Psyche und Stressempfinden gefunden werden - ein klares Ergebnis trotz des explorativen Vorgehens. Hinsichtlich der Interpretation der graphischen Betrachtungen dieser Arbeit ist zu beachten, dass diese lediglich der Plausibilitätsprüfung und der Hypothesengenerierung dienen können. Eine weitere Limitation stellten die doch sehr unterschiedlichen Therapiedauern auf der Station 9 des Asklepios Fachklinikums Göttingen sowie in anderen psychiatrischen Kliniken dar. Ursprünglich war eine einheitlichere Therapiedauer und eine überwiegend auf der Station 9 stattfindende Therapie erwartet worden. Hier zeigt sich einmal mehr die schwierige Planbarkeit von Follow-up-Studien im psychiatrischen Bereich. Eine einheitlichere Therapiedauer wäre erreicht worden, wenn die Patientinnen erst nach der Diagnostikphase rekrutiert worden wären. So wäre jedoch eine Messung ohne Therapieeffekt nicht möglich gewesen. Eine zweite Möglichkeit wäre ein Ausschluss der Patientinnen mit einer geringeren Therapiedauer gewesen, wodurch die Generalisierbarkeit jedoch wiederum vermindert gewesen wäre. Eine überzeugende Lösung wäre eine Subgruppenbildung gewesen, die jedoch aufgrund der geringen Teilnehmerzahl nicht realisierbar war.

Die Patientinnen waren durch eine Vielzahl an psychiatrischen Komorbiditäten und die häufige Einnahme von Medikamenten gekennzeichnet. Ein Ausschluss aufgrund von psychiatrischen Komorbiditäten und Medikation erfolgte nicht. Auch für somatische Erkrankungen waren die Ausschlusskriterien eng gefasst. Hier findet sich ein grundsätzliches Spannungsfeld der Forschung. Ein Einfluss all dieser Faktoren auf die Ergebnisse ist nicht auszuschließen und ein großzügiger Ausschluss hätte spezifischere Rückschlüsse auf die PTBS-typischen Pathologien ermöglicht. Gleichzeitig gehören jedoch gerade die Komorbiditäten und die Medikation untrennbar zum Krankheitsbild der komplexen Traumafolgestörungen. Ein Ausschluss aufgrund dieser Faktoren geht daher immer mit einer deutlich eingeschränkten Generalisierbarkeit einher. Ebenso können kardiovaskuläre Erkrankungen die stressphysiologische Messung beeinträchtigen. Aufgrund des erhöhten kardiovaskulären Risikos der PTBS ist aber der hohe Anteil an kardiovaskulären Erkrankungen auch ein Charakteristikum dieser Patientengruppe. Ein Ausschuss wäre wiederum einer Selektion gleichgekommen. Eine Subgruppenbildung hätte eine Lösung darstellen können, war aber aufgrund der Größe der Kohorte nicht möglich. Um dennoch der Problematik gerecht zu werden, wurde eine Sensitivitätsanalyse zur Betablockermedikation durchgeführt, da hier der Verdacht auf eine Konfundierung bestand, der teils bestätigt werden konnte. Des Weiteren wäre eine Kontrollgruppe im Follow-up wünschenswert gewesen - auch hier zeigten sich jedoch Grenzen in der Umsetzbarkeit. Insbesondere eine PTBS-Kontrollgruppe ohne Therapie hätte Rückschlüsse auf zeitliche Effekte ermöglicht und den Einfluss der Messwiederholung offengelegt. Eine solche Kontrollgruppe ist jedoch aufgrund organisatorischer (Rekrutierung) und ethischer Gesichtspunkte schwierig umsetzbar. Multizenterstudien könnten die Rekrutierungsproblematik lösen, sodass sie die Erforschung der in dieser Studie aufgeworfenen Fragestellungen durch größere Kohorten, Subgruppen und Kontrollgruppen ermöglichen könnten. 


\section{$7 \quad$ Zusammenfassung}

Die autonome Dysbalance steht in Verdacht, an der Genese des erhöhten kardiovaskulären Risikos der PTBS beteiligt zu sein. Daher ist es von großem klinischem Interesse, ob das ANS durch die etablierten Therapieregime erreicht wird. Die Datenlage zu der autonomen Dysbalance ist bisweilen inkonsistent: Während bei traumaassoziierten Stressoren meist eine Hyperreaktivität beschrieben wurde, konnte vor allem bei traumaunabhängigen Stressoren eine allgemeine Hyporeaktivität bilanziert werden. Aktuelle Studien vermuten zunehmend eine vagale Hypoaktivität und Hyporeagibilität als zugrundeliegenden Pathomechanismus für diese stressphysiologischen Auffälligkeiten der PTBS. Bei den seltenen psychophysiologischen Therapieevaluationen wurde bislang der Parasympathikus vernachlässigt, sodass unklar ist, ob eine PTBS-Therapie auch einen Einfluss auf die vagale Hyporeagibilität nimmt. Mit der Fokussierung auf eine weibliche Kohorte mit komplexen Traumafolgestörungen wurde ein weiterer Schwerpunkt dieser Arbeit gesetzt.

In der vorliegenden Studie wurde der Verlauf des subjektiven Stressempfindens, des psychometrischen Befunds sowie der kardiovaskulären Stressreagibilität bei 26 Patientinnen mit komplexen Traumafolgestörungen evaluiert. Mithilfe des Task Force ${ }^{\circledR}$ Monitors, eines kardiovaskulären Funktionsmonitors, wurden die Parameter HR, sBP, TPRI, PEP und BRS während eines mehrphasigen Stresstests vor und nach einer Therapie aufgezeichnet. Der Stresstest bestand neben Ruhephasen und einer Erholungsphase mit Musik aus den beiden fünfminutigen Stressphasen Rechnen und Babyschreien. Die Rekrutierung der Probandinnen erfolgte auf der Station 9 des Asklepios Fachklinikums Göttingen, wo auch eine umfangreiche Diagnostik zu PTBS, BPS und weiteren Komorbiditäten zum Zeitpunkt Baseline stattfand. In den im Mittel drei Jahren zwischen der Baseline- und Follow-up-Messung fand eine speziell auf diese Patientengruppe ausgerichtete PTBS-Therapie auf ebenjener Station statt, wobei die individuelle Therapiedauer stark schwankte und auch andere psychiatrische Therapien von den Patientinnen in Anspruch genommen wurden.

Für das subjektive Stressempfinden konnte eine Befundbesserung im Follow-up bilanziert werden. Dagegen besserte sich unter den psychometrischen Fragebögen einzig die depressive Symptomatik (BDI) signifikant. SCL-90-R und IES-R zeigten ebenfalls einen positiven Trend mit überwiegend niedrigeren Werten im Follow-up und einzelnen signifikant gebesserten Skalen. Die dissoziative (FDS) sowie die BPS-Symptomatik (BPI, BSL) waren weitgehend unverändert bzw. zeigten zum Teil sogar eine Verschlechterung an. Im ANS spiegelte sich dagegen kein Therapieeffekt wider. Die Reaktivität auf Rechnen und Babyschreien sowie die Recovery und die allgemeine Reagibilität waren für die verschiedenen Parameter weitgehend unverändert. Zudem konnten nur wenige Assoziationen zwischen den Veränderungen der stressphysiologischen Reagibilität, des Stressempfindens oder der Psychometrie sowie der Therapiedauer gefunden werden. Der weitgehend unveränderte stressphysiologische Befund impliziert eine Reproduktion der Ergebnisse der beiden Dissertationen zu der Baseline-Messung (Albrecht 2013, Bornschein 2014). Durch die Replikation 
der vagalen und allgemeinen Hyporeaktivität auf die Stressoren Rechnen und Babyschreien konnte die Generalisierbarkeit der Hyporeagibilität der PTBS bei verschiedenen Stressoren weiter untermauert werden. Auch die zeitliche Stabilität dieser Reaktionsmuster konnte gezeigt werden.

Die vorliegende Studie deutet somit darauf hin, dass die allgemeine sowie die vagale Hyporeagibilität bei den komplexen Traumafolgestörungen unberührt von etablierten Therapieregimen bleiben. Neben einer Therapieresistenz des vagalen Systems oder der autonomen Hyporeagibilität im Allgemeinen könnten auch Verzerrungen durch Medikation, Komorbiditäten und den zeitlichen Abstand des Follow-ups den unveränderten stressphysiologischen Befund mitbedingen. Es besteht eine Diskrepanz zu den wenigen vorausgegangenen Studien zu der psychophysiologischen Therapieevaluation bei der PTBS. Hier wurden jedoch in der Regel andere, traumaassoziierte Stressoren und damit nicht die Hyporeagibilität betrachtet, auch der Parasympathikus wurde unzureichend beachtet. Zudem konzentriert sich die vorliegende Studie auf die komplexen Traumafolgestörungen, die sich vielfältig von der in der Regel betrachteten „klassischen“ PTBS unterscheiden und als schwerer therapierbar gelten. Bezüglich der Komorbiditäten bleibt insbesondere unklar, welchen Anteil die Depression an den autonomen Auffälligkeiten der PTBS haben könnte. Bemerkenswert waren zudem die fehlenden Assoziationen zwischen Stressempfinden, Psyche und Stressphysiologie. So ging der Stressor Babyschreien mit einem starken subjektiven Stresserleben einher, obwohl die physiologische Stressreaktion gering ausfiel.

Zusammenfassend konnten keine Hinweise für eine Therapiewirkung auf die autonome Dysbalance gefunden werden. Dieses Ergebnis wirft verschiedene Fragen auf - nicht zuletzt, ob eine mögliche Therapieresistenz der stressphysiologischen Hyporeagibilität durch spezifische, auf das ANS abzielende Therapiekonzepte verhindert werden könnte. In jedem Fall konnte gezeigt werden, wie entscheidend eine umfassende Therapieevaluation ist. Verschiedene Stressoren müssen eingesetzt werden, und neben der sympathischen Regulation verdient auch das vagale System Beachtung. Zudem gilt es Stressempfinden, Psychometrie und Stressphysiologie in gleichem Maße zu berücksichtigen. Nur so kann eine Therapieevaluation der Komplexität einer Erkrankung wie der PTBS gerecht werden. 


\section{Anhang}

\subsection{Tabellen zur Testung auf systematische Unterschiede zwischen den Gruppen „Follow-up“ und „Nur Baseline“}

Tabelle A1: Unabhängiger t-Test zu der Frage nach systematischen Unterschieden zwischen der Gruppe „Nur Teilnahme an der Baseline-Messung“ (= „Nur Baseline“, $\mathrm{N}=28$ ) und der Gruppe „Teilnahme an Baseline und Follow-up“ (= „Follow-up“, $N=26$ ), s. hierzu auch Kap. 4.2.2. Angegeben sind die Mittelwerte und Standardabweichungen der beiden Gruppen sowie die Teststatistik t, die Freiheitsgrade df und die Signifikanz $p$ (zweiseitig). Getestet wurden verschiedene, mindestens intervallskalierte Variablen des allgemeinen Fragebogens, des Stressempfindens, der psychometrischen Fragebögen sowie kardiovaskuläre Parameter der Phase Ruhe 1. Die Variable Alkoholkonsum zeigte sich im Levene-Test signifikant, sodass im t-Test die Werte für eine angenommene Varianzinhomogenität herangezogen wurden (mit* gekennzeichnet).

\begin{tabular}{|c|c|c|c|c|c|c|c|}
\hline \multirow{2}{*}{$\begin{array}{c}\text { Unabhängiger } \\
\text { t-Test }\end{array}$} & \multicolumn{2}{|c|}{ „Nur Baseline“ } & \multicolumn{2}{|c|}{ „Follow-up“ } & \multirow{2}{*}{$\mathbf{t}$} & \multirow{2}{*}{ df } & \multirow{2}{*}{$\begin{array}{l}\text { Signifikanz } \\
\text { (zweiseitig) }\end{array}$} \\
\hline & $M$ & $S D$ & $M$ & $S D$ & & & \\
\hline Alter & 40,4 & 8,5 & 42,5 & 8,9 & $-0,9$ & 52 & 0,38 \\
\hline Größe $[\mathrm{cm}]$ & 166,1 & 5,7 & 165,1 & 8,0 & 0,5 & 52 & 0,60 \\
\hline Gewicht [kg] & 75,4 & 19,3 & 75,8 & 16,8 & $-0,1$ & 51 & 0,94 \\
\hline $\mathrm{BMI}\left[\mathrm{kg} / \mathrm{m}^{2}\right]$ & 27,5 & 7,1 & 27,8 & 5,9 & $-0,2$ & 51 & 0,86 \\
\hline Anzahl Kinder & 1,5 & 1,3 & 1,2 & 1,3 & 1,1 & 52 & 0,28 \\
\hline $\begin{array}{l}\text { Alkohol [drinks } \\
\text { per week] }\end{array}$ & 1,3 & 2,6 & 4,0 & 8,6 & $-1,5^{*}$ & $29,4^{*}$ & $0,13^{*}$ \\
\hline $\begin{array}{c}\text { Rauchen } \\
\text { [packyears] }\end{array}$ & 14,2 & 15,3 & 11,8 & 18,3 & 0,5 & 52 & 0,59 \\
\hline $\begin{array}{c}\text { Stressempfinden } \\
\text { Ruhe } 1\end{array}$ & 2,9 & 1,7 & 3,7 & 1,8 & $-1,7$ & 52 & 0,10 \\
\hline $\begin{array}{l}\text { Stressempfinden } \\
\text { Rechnen }\end{array}$ & 6,1 & 1,9 & 6,5 & 2,0 & $-0,8$ & 52 & 0,43 \\
\hline $\begin{array}{c}\text { Stressempfinden } \\
\text { Babyschreien }\end{array}$ & 6,1 & 2,7 & 6,5 & 2,5 & $-0,6$ & 52 & 0,58 \\
\hline $\begin{array}{c}\text { Stressempfinden } \\
\text { Musik }\end{array}$ & 4,0 & 3,3 & 4,5 & 2,6 & $-0,6$ & 52 & 0,53 \\
\hline $\begin{array}{c}\text { GSI des } \\
\text { SCL-90-R }\end{array}$ & 1,6 & 0,8 & 1,7 & 0,7 & $-0,5$ & 46 & 0,59 \\
\hline BDI & 28,6 & 12,5 & 29,0 & 9,1 & $-0,2$ & 45 & 0,88 \\
\hline IES-R Intrusion & 24,4 & 7,9 & 24,9 & 7,4 & 0,2 & 42 & 0,82 \\
\hline IES-R Vermeidung & 27,0 & 6,7 & 25,5 & 10,4 & $-0,5$ & 42 & 0,59 \\
\hline $\begin{array}{c}\text { IES-R } \\
\text { Übererregung }\end{array}$ & 23,7 & 8,5 & 23,6 & 6,8 & $-0,04$ & 42 & 0,97 \\
\hline BPI Cut-20 & 11,1 & 4,4 & 11,3 & 4,4 & $-0,2$ & 46 & 0,87 \\
\hline BSL Gesamtwert & 156,7 & 67,7 & 148,1 & 62,3 & 0,46 & 46 & 0,65 \\
\hline
\end{tabular}


Fortsetzung Tabelle A1

\begin{tabular}{|c|c|c|c|c|c|c|c|}
\hline & \multicolumn{2}{|c|}{ „Nur Baseline“ } & \multicolumn{2}{|c|}{ „Follow-up“ } & \multirow{2}{*}{$\mathbf{t}$} & \multirow{2}{*}{ df } & \multirow{2}{*}{$\begin{array}{l}\text { Signifikanz } \\
\text { (zweiseitig) }\end{array}$} \\
\hline & $M$ & $S D$ & $M$ & $S D$ & & & \\
\hline FDS Gesamtwert & 19,2 & 14,8 & 20,1 & 14,1 & $-0,2$ & 46 & 0,83 \\
\hline DES Gesamtwert & 22,3 & 17,4 & 22,8 & 15,8 & $-0,1$ & 46 & 0,92 \\
\hline $\begin{array}{l}\text { HF Ruhe } 1 \\
\text { [bpm] }\end{array}$ & 75,1 & 8,8 & 77,8 & 11,4 & 1,0 & 50 & 0,34 \\
\hline $\begin{array}{l}\text { sBP Ruhe } 1 \\
{[\mathrm{mmHg}]}\end{array}$ & 113,9 & 11,6 & 115,0 & 13,2 & $-0,3$ & 50 & 0,74 \\
\hline $\begin{array}{l}\text { TPRI Ruhe } 1 \text { [ } \\
\text { dyn*s*m² } / \mathrm{cm}^{5} \text { ] }\end{array}$ & 2355,6 & 648,8 & 2651,5 & 857,8 & $-1,4$ & 50 & 0,16 \\
\hline PEP Ruhe 1 [ms] & 115,8 & 11,6 & 117,9 & 11,4 & $-0,6$ & 50 & 0,53 \\
\hline $\begin{array}{l}\text { BRS Ruhe } 1 \\
{[\mathrm{~ms} / \mathrm{mmHg}]}\end{array}$ & 12,4 & 9,3 & 9,7 & 4,0 & 1,4 & 50 & 0,18 \\
\hline
\end{tabular}

Tabelle A2: Chi-Quadrat-Test zu der Frage nach systematischen Unterschieden zwischen der Gruppe „Nur Teilnahme an der Baseline-Messung“ (= „Nur Baseline“, N = 28) und der Gruppe „Teilnahme an Baseline und Follow-up“ (= „Follow-up“, $\mathrm{N}=26$ ), s. hierzu auch Kap. 4.2.2. Getestet wurden verschiedene, kategoriale Variablen der soziodemographischen und klinischen Daten. Angegeben sind entsprechend dem Chi-Quadrat nach Pearson $\chi^{2}$, die Freiheitsgrade df und die zweiseitige Signifikanz $p$. Es muss teilweise eine eingeschränkte Aussagekraft beachtet werden, da bei einigen Variablen die erwartete Anzahl von über fünf pro Kategorie nicht erreicht wurde.

\begin{tabular}{|l|c|c|c|}
\hline \multicolumn{1}{|c|}{ Chi-Quadrat nach Pearson } & $\chi^{2}$ & df & $\begin{array}{c}\text { Signifikanz } \\
\text { (zweiseitig) }\end{array}$ \\
\hline Familienstand & 1,6 & 3 & 0,67 \\
\hline Schulabschluss & 8,1 & 5 & 0,15 \\
\hline Ausbildungsabschluss & 4,9 & 6 & 0,56 \\
\hline Abgeschlossene Ausbildung & 0,4 & 1 & 0,51 \\
\hline Erwerbstätigkeit (Stunden pro Woche) & 2,3 & 3 & 0,52 \\
\hline Einteilung einfache oder komplexe PTBS & 1,3 & 1 & 0,25 \\
\hline Einteilung PTBS: Typ 1, Typ 2 oder Typ 1 + 2 & 6,0 & 2 & 0,051 \\
\hline Vorliegen einer BPS oder sonstiger & 0,3 & 2 & 0,84 \\
\hline Persönlichkeitsstörung & 0,01 & 1 & 0,93 \\
\hline Vorliegen einer dissoziativen Störung & 1,9 & 1 & 0,16 \\
\hline Vorliegen einer depressiven Störung & 0,6 & 1 & 0,44 \\
\hline Vorliegen einer somatoformen Störung & 0,2 & 1 & 0,63 \\
\hline Vorliegen einer Angst- bzw. Panikstörung & 2,1 & 1 & 0,15 \\
\hline Vorliegen einer Essstörung & 0,002 & 1 & 0,97 \\
\hline Selbstverletzendes Verhalten & 2,9 & 3 & 0,40 \\
\hline Vorliegen/Anzahl von Abhängigkeitserkrankungen & 2,6 & 1 & 0,11 \\
\hline Rauchen & 0,2 & 1 & 0,63 \\
\hline Alkoholabhängigkeit & 0,3 & 1 & 0,57 \\
\hline Medikamentenabusus & & & \\
\hline
\end{tabular}


Tabelle A3: Chi-Quadrat-Test zu der Frage nach systematischen Unterschieden zwischen der Gruppe „Nur Teilnahme an der Baseline-Messung“ (= „Nur Baseline“, N = 28) und der Gruppe „Teilnahme an Baseline und Follow-up“ (= „Follow-up“, $N=26$ ), s. hierzu auch Kap. 4.2.2. Da im ursprünglichen Chi-Quadrat-Test einige Variablen nicht die erwartete Anzahl von über fünf pro Kategorie erreichten, wurden hier Kategorien, soweit dies sinnvoll möglich war, vor der Testung zusammengefasst. So wurde die Anzahl der Kategorien vermindert und damit die Anzahl der Fälle pro Kategorie erhöht. Getestet wurden verschiedene kategoriale Variablen der soziodemographischen und klinischen Daten. Angegeben sind entsprechend dem Chi-Quadrat nach Pearson $\chi^{2}$, die Freiheitsgrade df und die zweiseitige Signifikanz $p$.

\begin{tabular}{|l|c|c|c|}
\hline \multicolumn{1}{|c|}{ Chi-Quadrat nach Pearson } & $\boldsymbol{\chi}^{\mathbf{2}}$ & $\mathbf{d f}$ & $\begin{array}{c}\text { Signifikanz } \\
\text { (zweiseitig) }\end{array}$ \\
\hline Vorliegen einer Erwerbstätigkeit & 1,5 & 1 & 0,22 \\
\hline Vorliegen einer Partnerschaft & 2,8 & 1 & 0,60 \\
\hline $\begin{array}{l}\text { Sport (mindestens eine halbe Stunde Sport pro } \\
\text { Woche) }\end{array}$ & 1,2 & 1 & 0,28 \\
\hline Einnahme Antihypertensiva (außer Betablockern) & 2,3 & 1 & 0,12 \\
\hline Einnahme Betablocker & 1,5 & 1 & 0,22 \\
\hline Einnahme anticholinerge Medikation & 0,3 & 1 & 0,57 \\
\hline Vorliegen einer Suchterkrankung & 2,7 & 1 & 0,10 \\
\hline
\end{tabular}




\subsection{Vergleichstabellen: Reactivity und Recovery ohne Ausschluss der Patientin E1}

Tabelle A4: Zum Vergleich mit Tabelle 15 („Reactivity Rechnen“), S. 70. Phasenmittelwerte und Standardabweichungen der Phasen Ruhe 1 und Rechnen (jeweils für Baseline und Follow-up) und eine 2 x 2 ANOVA für Messwiederholungen für die Parameter HR in [bpm], sBP in [mmHg], TPRI in [dyn*s* ${ }^{2} / \mathrm{cm}^{5}$ ], PEP in [ms] und BRS in [ms/mmHg] - alles ohne Ausschluss von Patientin E1 (s. Kap. 4.2.5).

\begin{tabular}{|c|c|c|c|c|c|c|c|}
\hline & & & $\begin{array}{c}\mathbf{H R} \\
(N=25) \\
\end{array}$ & $\begin{array}{c}\mathbf{s B P} \\
(N=25) \\
\end{array}$ & $\begin{array}{c}\text { TPRI } \\
(N=25) \\
\end{array}$ & $\begin{array}{c}\text { PEP } \\
(N=22) \\
\end{array}$ & $\begin{array}{c}\text { BRS } \\
(N=25) \\
\end{array}$ \\
\hline \multicolumn{8}{|c|}{ Mittelwerte mit Standardabweichung über die Phasen } \\
\hline \multirow{4}{*}{ Ruhe 1} & \multirow{2}{*}{ Baseline } & $M$ & 77,8 & 115,0 & 2651,5 & 117,2 & 9,7 \\
\hline & & $S D$ & 11,4 & 13,2 & 857,8 & 11,9 & 4,0 \\
\hline & \multirow{2}{*}{$\begin{array}{l}\text { Follow- } \\
\text { up }\end{array}$} & M & 74,2 & 115,8 & 3038,2 & 110,3 & 10,1 \\
\hline & & $S D$ & 6,4 & 16,4 & 952,8 & 9,9 & 6,1 \\
\hline \multirow{4}{*}{ Rechnen } & \multirow{2}{*}{ Baseline } & $M$ & 81,1 & 122,0 & 2658,8 & 115,5 & 8,7 \\
\hline & & $S D$ & 11,4 & 12,4 & 923,4 & 11,5 & 3,6 \\
\hline & \multirow{2}{*}{$\begin{array}{c}\text { Follow- } \\
\text { up }\end{array}$} & $M$ & 77,1 & 121,7 & 3017,3 & 110,3 & 8,9 \\
\hline & & $S D$ & 6,9 & 16,4 & 865,9 & 8,3 & 3,8 \\
\hline \multicolumn{8}{|c|}{$2 \times 2$ ANOVA für Messwiederholung zur Reactivity Rechnen } \\
\hline \multirow{3}{*}{ Messzeitpunkt } & \multicolumn{2}{|c|}{ Teststatistik F } & 3,9 & 0,005 & 4,9 & 3,1 & 0,1 \\
\hline & \multicolumn{2}{|c|}{ Freiheitsgrade } & $1 ; 24$ & $1 ; 24$ & $1 ; 24$ & $1 ; 21$ & $1 ; 24$ \\
\hline & \multicolumn{2}{|c|}{ Signifikanz } & 0,06 & 0,94 & $<0,05$ & 0,09 & 0,77 \\
\hline \multirow{3}{*}{$\begin{array}{l}\text { Stressor } \\
\text { Rechnen }\end{array}$} & \multicolumn{2}{|c|}{ Teststatistik F } & 53,6 & 34,5 & 0,3 & 0,9 & 7,1 \\
\hline & \multicolumn{2}{|c|}{ Freiheitsgrade } & $1 ; 24$ & $1 ; 24$ & $1 ; 24$ & $1 ; 21$ & $1 ; 24$ \\
\hline & \multicolumn{2}{|c|}{ Signifikanz } & $<0,001$ & $<0,001$ & 0,87 & 0,36 & $<0,05$ \\
\hline \multirow{3}{*}{$\begin{array}{l}\text { Wechsel- } \\
\text { wirkung } \\
\text { Messzeitpunkt } \\
\text { x Stressor }\end{array}$} & \multicolumn{2}{|c|}{ Teststatistik $F$} & 0,4 & 0,3 & 0,1 & 1,1 & 0,1 \\
\hline & \multicolumn{2}{|c|}{ Freiheitsgrade } & $1 ; 24$ & $1 ; 24$ & $1 ; 24$ & $1 ; 21$ & $1 ; 24$ \\
\hline & \multicolumn{2}{|c|}{ Signifikanz } & 0,54 & 0,60 & 0,71 & 0,30 & 0,79 \\
\hline
\end{tabular}


Tabelle A5: Zum Vergleich mit Tabelle 16 („Reactivity Babyschreien“), S. 71. Phasenmittelwerte und Standardabweichungen der Phasen Ruhe 1 und Babyschreien (jeweils für Baseline und Follow-up) und eine 2 x 2 ANOVA für Messwiederholungen für die Parameter HR in [bpm], sBP in [mmHg], TPRI in $\left[\mathrm{dyn}^{*} \mathrm{~s}^{*} \mathrm{~m}^{2} / \mathrm{cm}^{5}\right]$, PEP in [ms] und BRS in [ms $/ \mathrm{mmHg}$ - alles ohne Ausschluss von Patientin E1 (s. Kap. 4.2.5).

\begin{tabular}{|c|c|c|c|c|c|c|c|}
\hline & & & $\begin{array}{c}\text { HR } \\
(N=25) \\
\end{array}$ & $\begin{array}{c}\mathbf{s B P} \\
(N=25) \\
\end{array}$ & $\begin{array}{c}\text { TPRI } \\
(N=24) \\
\end{array}$ & $\begin{array}{c}\text { PEP } \\
(N=22) \\
\end{array}$ & $\begin{array}{c}\text { BRS } \\
(N=25) \\
\end{array}$ \\
\hline \multicolumn{8}{|c|}{ Mittelwerte mit Standardabweichung über die Phasen } \\
\hline \multirow{4}{*}{ Ruhe 1} & \multirow{2}{*}{ Baseline } & M & 77,8 & 115,0 & 2624,1 & 117,2 & 9,7 \\
\hline & & $S D$ & 11,4 & 13,2 & 865,0 & 11,9 & 4,0 \\
\hline & \multirow{2}{*}{$\begin{array}{c}\text { Follow- } \\
\text { up }\end{array}$} & $M$ & 74,2 & 115,8 & 3083,9 & 110,3 & 10,1 \\
\hline & & $S D$ & 6,4 & 16,4 & 944,9 & 9,9 & 6,1 \\
\hline \multirow{4}{*}{ Babyschreien } & \multirow{2}{*}{ Baseline } & $M$ & 77,6 & 120,6 & 2792,4 & 115,8 & 10,5 \\
\hline & & $S D$ & 12,7 & 19,4 & 940,8 & 11,8 & 6,0 \\
\hline & \multirow{2}{*}{$\begin{array}{c}\text { Follow- } \\
\text { up }\end{array}$} & $M$ & 73,0 & 117,8 & 3251,3 & 114,1 & 10,8 \\
\hline & & $S D$ & 7,7 & 17,8 & 1043,9 & 10,8 & 5,0 \\
\hline \multicolumn{8}{|c|}{$2 \times 2$ ANOVA für Messwiederholung zur Reactivity Babyschreien } \\
\hline \multirow{3}{*}{ Messzeitpunkt } & \multicolumn{2}{|c|}{ Teststatistik F } & 4,1 & 0,1 & 8,0 & 1,4 & 0,1 \\
\hline & \multicolumn{2}{|c|}{ Freiheitsgrade } & $1 ; 24$ & $1 ; 24$ & $1 ; 23$ & $1 ; 21$ & $1 ; 24$ \\
\hline & \multicolumn{2}{|c|}{ Signifikanz } & 0,05 & 0,79 & $<0,01$ & 0,25 & 0,77 \\
\hline \multirow{3}{*}{$\begin{array}{c}\text { Stressor } \\
\text { Babyschreien }\end{array}$} & \multicolumn{2}{|c|}{ Teststatistik F } & 0,5 & 3,8 & 6,1 & 1,3 & 1,7 \\
\hline & \multicolumn{2}{|c|}{ Freiheitsgrade } & $1 ; 24$ & $1 ; 24$ & $1 ; 23$ & $1 ; 21$ & $1 ; 24$ \\
\hline & \multicolumn{2}{|c|}{ Signifikanz } & 0,50 & 0,06 & $<0,05$ & 0,27 & 0,20 \\
\hline \multirow{3}{*}{$\begin{array}{c}\text { Wechsel- } \\
\text { wirkung } \\
\text { Messzeitpunkt } \\
\text { x Stressor }\end{array}$} & \multicolumn{2}{|c|}{ Teststatistik F } & 1,7 & 0,6 & 0,0 & 5,3 & 0,01 \\
\hline & \multicolumn{2}{|c|}{ Freiheitsgrade } & $1 ; 24$ & $1 ; 24$ & $1 ; 23$ & $1 ; 21$ & $1 ; 24$ \\
\hline & \multicolumn{2}{|c|}{ Signifikanz } & 0,21 & 0,44 & 0,995 & $<0,05$ & 0,91 \\
\hline
\end{tabular}


Tabelle A6: Zum Vergleich mit Tabelle 17 („Recovery Rechnen“), S. 72. Phasenmittelwerte und Standardabweichungen der Phasen Rechnen und Entspannungsmusik (jeweils für Baseline und Follow-up) und eine $2 \times 2$ ANOVA für Messwiederholungen für die Parameter HR in [bpm], sBP in $\left[\mathrm{mmHg}\right.$, TPRI in [dyn*s* $\left.\mathrm{m}^{2} / \mathrm{cm}^{5}\right]$, PEP in [ms] und BRS in $[\mathrm{ms} / \mathrm{mmHg}$ - alles ohne Ausschluss von Patientin E1 (s. Kap. 4.2.5).

\begin{tabular}{|c|c|c|c|c|c|c|c|}
\hline & & & $\begin{array}{c}\mathbf{H R} \\
(N=24) \\
\end{array}$ & $\begin{array}{c}\mathbf{s B P} \\
(N=24) \\
\end{array}$ & $\begin{array}{c}\text { TPRI } \\
(N=23) \\
\end{array}$ & $\begin{array}{c}\text { PEP } \\
(N=21) \\
\end{array}$ & $\begin{array}{c}\text { BRS } \\
(N=24) \\
\end{array}$ \\
\hline \multicolumn{8}{|c|}{ Mittelwerte mit Standardabweichung über die Phasen } \\
\hline \multirow{4}{*}{ Rechnen } & \multirow{2}{*}{ Baseline } & $M$ & 80,9 & 121,5 & 2682,2 & 116,3 & 8,6 \\
\hline & & $S D$ & 11,6 & 12,4 & 937,1 & 11,2 & 3,6 \\
\hline & \multirow{2}{*}{$\begin{array}{c}\text { Follow- } \\
\text { up }\end{array}$} & $M$ & 77,0 & 120,0 & 3028,9 & 110,6 & 8,7 \\
\hline & & $S D$ & 7,1 & 14,2 & 891,1 & 8,4 & 3,8 \\
\hline \multirow{4}{*}{$\begin{array}{l}\text { Entspannungs- } \\
\text { musik }\end{array}$} & \multirow{2}{*}{ Baseline } & $M$ & 74,7 & 117,5 & 2838,3 & 119,4 & 11,3 \\
\hline & & $S D$ & 11,4 & 15,7 & 947,8 & 10,2 & 6,4 \\
\hline & \multirow{2}{*}{$\begin{array}{c}\text { Follow- } \\
\text { up }\end{array}$} & $M$ & 71,8 & 117,0 & 3274,9 & 114,3 & 11,2 \\
\hline & & $S D$ & 7,7 & 14,9 & 1042,6 & 10,4 & 6,2 \\
\hline \multicolumn{8}{|c|}{2 × 2 ANOVA für Messwiederholung zur Recovery Rechnen } \\
\hline \multirow{3}{*}{ Messzeitpunkt } & \multicolumn{2}{|c|}{ Teststatistik $F$} & 3,2 & 0,1 & 5,4 & 2,6 & 0,0 \\
\hline & \multicolumn{2}{|c|}{ Freiheitsgrade } & $1 ; 23$ & $1 ; 23$ & $1 ; 22$ & $1 ; 20$ & $1 ; 23$ \\
\hline & \multicolumn{2}{|c|}{ Signifikanz } & 0,08 & 0,75 & $<0,05$ & 0,12 & 0,99 \\
\hline \multirow{3}{*}{$\begin{array}{l}\text { Recovery } \\
\text { Rechnen }\end{array}$} & \multicolumn{2}{|c|}{ Teststatistik $F$} & 74,6 & 7,4 & 19,6 & 10,0 & 19,5 \\
\hline & \multicolumn{2}{|c|}{ Freiheitsgrade } & $1 ; 23$ & $1 ; 23$ & $1 ; 22$ & $1 ; 20$ & $1 ; 23$ \\
\hline & \multicolumn{2}{|c|}{ Signifikanz } & $<0,001$ & $<0,05$ & $<0,001$ & $<0,01$ & $<0,001$ \\
\hline \multirow{3}{*}{$\begin{array}{c}\text { Wechsel- } \\
\text { wirkung } \\
\text { Messzeitpunkt } \\
\text { x Recovery }\end{array}$} & \multicolumn{2}{|c|}{ Teststatistik F } & 2,1 & 0,2 & 1,1 & 0,1 & 0,1 \\
\hline & \multicolumn{2}{|c|}{ Freiheitsgrade } & $1 ; 23$ & $1 ; 23$ & $1 ; 22$ & $1 ; 20$ & $1 ; 23$ \\
\hline & \multicolumn{2}{|c|}{ Signifikanz } & 0,16 & 0,66 & 0,31 & 0,80 & 0,82 \\
\hline
\end{tabular}


Tabelle A7: Zum Vergleich mit Tabelle 18 („Recovery Babyschreien“), S. 73. Phasenmittelwerte und Standardabweichungen der Phasen Babyschreien und Entspannungsmusik (jeweils für Baseline und Follow-up) und eine 2 x 2 ANOVA für Messwiederholungen für die Parameter HR in [bpm], sBP in $[\mathrm{mmHg}]$, TPRI in $\left[\mathrm{dyn}^{*} \mathrm{~s}^{*} \mathrm{~m}^{2} / \mathrm{cm}^{5}\right]$, PEP in [ms] und BRS in [ms $/ \mathrm{mmHg}$ - alles ohne Ausschluss von Patientin E1 (s. Kap. 4.2.5).

\begin{tabular}{|c|c|c|c|c|c|c|c|}
\hline & & & $\begin{array}{c}\mathbf{H R} \\
(N=24) \\
\end{array}$ & $\begin{array}{c}\mathbf{s B P} \\
(N=24) \\
\end{array}$ & $\begin{array}{c}\text { TPRI } \\
(N=23) \\
\end{array}$ & $\begin{array}{c}\text { PEP } \\
(N=21) \\
\end{array}$ & $\begin{array}{c}\text { BRS } \\
(N=24) \\
\end{array}$ \\
\hline \multicolumn{8}{|c|}{ Mittelwerte mit Standardabweichung über die Phasen } \\
\hline \multirow{4}{*}{ Babyschreien } & \multirow{2}{*}{ Baseline } & $M$ & 76,8 & 119,7 & 2837,4 & 116,6 & 10,4 \\
\hline & & $S D$ & 12,3 & 19,3 & 935,2 & 11,5 & 6,2 \\
\hline & \multirow{2}{*}{$\begin{array}{c}\text { Follow- } \\
\text { up }\end{array}$} & $M$ & 72,4 & 116,3 & 3256,9 & 113,9 & 10,9 \\
\hline & & $S D$ & 7,3 & 16,4 & 1067,0 & 11,1 & 5,0 \\
\hline \multirow{4}{*}{$\begin{array}{l}\text { Entspannungs- } \\
\text { musik }\end{array}$} & \multirow{2}{*}{ Baseline } & $M$ & 74,7 & 117,5 & 2838,3 & 119,4 & 11,3 \\
\hline & & $S D$ & 11,4 & 15,7 & 947,8 & 10,2 & 6,4 \\
\hline & \multirow{2}{*}{$\begin{array}{c}\text { Follow- } \\
\text { up }\end{array}$} & $M$ & 71,8 & 117,0 & 3274,9 & 114,3 & 11,2 \\
\hline & & $S D$ & 7,7 & 14,9 & 1042,6 & 10,4 & 6,2 \\
\hline \multicolumn{8}{|c|}{$2 \times 2$ ANOVA für Messwiederholung zur Recovery Babyschreien } \\
\hline \multirow{3}{*}{ Messzeitpunkt } & \multicolumn{2}{|c|}{ Teststatistik $F$} & 3,4 & 0,2 & 5,9 & 1,1 & 0,02 \\
\hline & \multicolumn{2}{|c|}{ Freiheitsgrade } & $1 ; 23$ & $1 ; 23$ & $1 ; 22$ & $1 ; 20$ & $1 ; 23$ \\
\hline & \multicolumn{2}{|c|}{ Signifikanz } & 0,08 & 0,62 & $<0,05$ & 0,30 & 0,89 \\
\hline \multirow{3}{*}{$\begin{array}{c}\text { Recovery } \\
\text { Babyschreien }\end{array}$} & \multicolumn{2}{|c|}{ Teststatistik $F$} & 3,9 & 0,2 & 0,03 & 2,8 & 2,3 \\
\hline & \multicolumn{2}{|c|}{ Freiheitsgrade } & $1 ; 23$ & $1 ; 23$ & $1 ; 22$ & $1 ; 20$ & $1 ; 23$ \\
\hline & \multicolumn{2}{|c|}{ Signifikanz } & 0,06 & 0,67 & 0,86 & 0,11 & 0,14 \\
\hline \multirow{3}{*}{$\begin{array}{c}\text { Wechsel- } \\
\text { wirkung } \\
\text { Messzeitpunkt } \\
\text { x Recovery }\end{array}$} & \multicolumn{2}{|c|}{ Teststatistik F } & 4,6 & 0,8 & 0,02 & 2,3 & 0,3 \\
\hline & \multicolumn{2}{|c|}{ Freiheitsgrade } & $1 ; 23$ & $1 ; 23$ & $1 ; 22$ & $1 ; 20$ & $1 ; 23$ \\
\hline & \multicolumn{2}{|c|}{ Signifikanz } & $<0,05$ & 0,37 & 0,88 & 0,15 & 0,59 \\
\hline
\end{tabular}




\subsection{Regressionsanalyse: Beta-Koeffizienten}

Tabelle A8: Regressionskoeffizienten der Regressionsanalyse „Therapiedauer gesamt“. Es wurde eine multivariate Regression mit den Differenzwerten (Baseline - Follow-up) des Stressempfindens bzw. der psychometrischen Fragebögen als abhängige Variable durchgeführt. Zur Adjustierung wurde der jeweilige Baseline-Wert des Stressempfindens bzw. des psychometrischen Fragebogens als ein Prädiktor gesetzt. Als zweiter Prädiktor ging die „Therapiedauer gesamt“ in die Gleichung ein. Angegeben wird der standardisierte Koeffizient Beta mit dem zugehörigen Signifikanzwert jeweils für die beiden Prädiktoren. Trotz Kontrolle der Baseline-Werte zeigt der Prädiktor „Therapiedauer gesamt“ durchgehend einen negativen Zusammenhang zu der abhängigen Variable. Dies widerspricht der Hypothese 6.

\begin{tabular}{|c|c|c|c|}
\hline Abhängige Variable & Prädiktoren & Beta & Signifikanz \\
\hline \multirow{2}{*}{$\begin{array}{c}\text { Differenz Stress } \\
\text { Ruhe } 1\end{array}$} & Stress Ruhe 1 (Baseline) & 0,52 & $<0,05$ \\
\hline & „Therapiedauer gesamt“ & $-0,22$ & 0,32 \\
\hline \multirow{2}{*}{ Differenz Stress Rechnen } & Stress Rechnen (Baseline) & 0,52 & $<0,01$ \\
\hline & „Therapiedauer gesamt“ & $-0,29$ & 0,09 \\
\hline \multirow{2}{*}{$\begin{array}{c}\text { Differenz Stress } \\
\text { Babyschreien }\end{array}$} & Stress Babyschreien (Baseline) & 0,52 & $<0,01$ \\
\hline & „Therapiedauer gesamt“ & $-0,16$ & 0,38 \\
\hline \multirow{2}{*}{ Differenz Stress Musik } & Stress Musik (Baseline) & 0,53 & $<0,01$ \\
\hline & „Therapiedauer gesamt“ & $-0,40$ & $<0,05$ \\
\hline \multirow{2}{*}{ Differenz GSI (SCL-90-R) } & GSI des SCL-90-R (Baseline) & 0,54 & $<0,01$ \\
\hline & „Therapiedauer gesamt" & $-0,69$ & $<0,01$ \\
\hline \multirow{2}{*}{ Differenz Summenwert BDI } & BDI (Baseline) & 0,55 & $<0,01$ \\
\hline & „Therapiedauer gesamt“ & $-0,67$ & $<0,001$ \\
\hline \multirow{2}{*}{ Differenz IES-R Intrusion } & IES-R Intrusion (Baseline) & 0,26 & 0,32 \\
\hline & „Therapiedauer gesamt" & $-0,48$ & 0,07 \\
\hline \multirow{2}{*}{$\begin{array}{l}\text { Differenz IES-R } \\
\text { Vermeidung }\end{array}$} & IES-R Vermeidung (Baseline) & 0,71 & $<0,01$ \\
\hline & „Therapiedauer gesamt“ & $-0,01$ & 0,97 \\
\hline \multirow{2}{*}{$\begin{array}{l}\text { Differenz IES-R } \\
\text { Übererregung }\end{array}$} & IES-R Übererregung (Baseline) & 0,69 & $<0,01$ \\
\hline & „Therapiedauer gesamt" & $-0,48$ & $<0,05$ \\
\hline \multirow{2}{*}{ Differenz BPI Cut-20 } & BPI Cut-20 (Baseline) & 0,56 & $<0,01$ \\
\hline & „Therapiedauer gesamt“ & $-0,52$ & $<0,01$ \\
\hline \multirow{2}{*}{ Differenz BSL Gesamtskala } & BSL Gesamtskala (Baseline) & 0,55 & $<0,01$ \\
\hline & „Therapiedauer gesamt“ & $-0,73$ & $<0,01$ \\
\hline \multirow{2}{*}{ Differenz FDS Gesamtskala } & FDS Gesamtskala (Baseline) & 0,46 & $<0,05$ \\
\hline & „Therapiedauer gesamt" & $-0,58$ & $<0,01$ \\
\hline \multirow{2}{*}{ Differenz DES Gesamtskala } & DES Gesamtskala (Baseline) & 0,46 & $<0,05$ \\
\hline & „Therapiedauer gesamt“ & $-0,56$ & $<0,01$ \\
\hline
\end{tabular}


Tabelle A9: Regressionskoeffizienten der Regressionsanalyse „Therapiedauer Station 9“. Es wurde eine multivariate Regression mit den Differenzwerten (Baseline - Follow-up) des Stressempfindens bzw. der psychometrischen Fragebögen als abhängige Variable durchgeführt. Zur Adjustierung wurde der jeweilige Baseline-Wert des Stressempfindens bzw. des psychometrischen Fragebogens als ein Prädiktor gesetzt. Als zweiter Prädiktor ging die „Therapiedauer Station 9“ in die Gleichung ein. Angegeben wird der standardisierte Koeffizient Beta mit dem zugehörigen Signifikanzwert jeweils für die beiden Prädiktoren. Trotz Kontrolle der Baseline-Werte zeigt der Prädiktor „Therapiedauer Station 9" fast durchgehend einen negativen Zusammenhang zu der abhängigen Variable. Dies widerspricht der Hypothese 6. Eine Ausnahme ist die Regression „Stress Ruhe 1“, hier erreicht der Prädiktor „Therapiedauer Station 9“ jedoch keine Signifikanz.

\begin{tabular}{|c|c|c|c|}
\hline Abhängige Variable & Prädiktoren & Beta & Signifikanz \\
\hline \multirow{2}{*}{$\begin{array}{l}\text { Differenz Stress } \\
\text { Ruhe } 1\end{array}$} & Stress Ruhe 1 (Baseline) & 0,36 & 0,15 \\
\hline & „Therapiedauer Station 9“ & 0,09 & 0,71 \\
\hline \multirow{2}{*}{ Differenz Stress Rechnen } & Stress Rechnen (Baseline) & 0,56 & $<0,01$ \\
\hline & „Therapiedauer Station 9“ & $-0,21$ & 0,23 \\
\hline \multirow{2}{*}{$\begin{array}{c}\text { Differenz Stress } \\
\text { Babyschreien }\end{array}$} & Stress Babyschreien (Baseline) & 0,53 & $<0,01$ \\
\hline & „Therapiedauer Station 9“ & $-0,04$ & 0,84 \\
\hline \multirow{2}{*}{ Differenz Stress Musik } & Stress Musik (Baseline) & 0,45 & $<0,05$ \\
\hline & „Therapiedauer Station 9“ & $-0,10$ & 0,64 \\
\hline \multirow{2}{*}{ Differenz GSI (SCL-90-R) } & GSI des SCL-90-R (Baseline) & 0,26 & 0,24 \\
\hline & „Therapiedauer Station 9“ & $-0,12$ & 0,59 \\
\hline \multirow{2}{*}{ Differenz Summenwert BDI } & BDI (Baseline) & 0,35 & 0,09 \\
\hline & „Therapiedauer Station 9“ & $-0,31$ & 0,13 \\
\hline \multirow{2}{*}{ Differenz IES-R Intrusion } & IES-R Intrusion (Baseline) & 0,10 & 0,64 \\
\hline & „Therapiedauer Station 9“ & $-0,45$ & $<0,05$ \\
\hline \multirow{2}{*}{$\begin{array}{c}\text { Differenz IES-R } \\
\text { Vermeidung }\end{array}$} & IES-R Vermeidung (Baseline) & 0,71 & $<0,001$ \\
\hline & „Therapiedauer Station 9“ & $-0,03$ & 0,84 \\
\hline \multirow{2}{*}{$\begin{array}{l}\text { Differenz IES-R } \\
\text { Übererregung }\end{array}$} & IES-R Übererregung (Baseline) & 0,52 & $<0,05$ \\
\hline & „Therapiedauer Station 9“ & $-0,27$ & 0,15 \\
\hline \multirow{2}{*}{ Differenz BPI Cut-20 } & BPI Cut-20 (Baseline) & 0,48 & $<0,05$ \\
\hline & „Therapiedauer Station 9“ & $-0,22$ & 0,30 \\
\hline \multirow{2}{*}{ Differenz BSL Gesamtskala } & BSL Gesamtskala (Baseline) & 0,25 & 0,24 \\
\hline & „Therapiedauer Station 9“ & $-0,32$ & 0,14 \\
\hline \multirow{2}{*}{ Differenz FDS Gesamtskala } & FDS Gesamtskala (Baseline) & 0,33 & 0,13 \\
\hline & „Therapiedauer Station 9“ & $-0,06$ & 0,76 \\
\hline \multirow{2}{*}{ Differenz DES Gesamtskala } & DES Gesamtskala (Baseline) & 0,32 & 0,14 \\
\hline & „Therapiedauer Station 9“ & $-0,12$ & 0,57 \\
\hline
\end{tabular}




\subsection{Vergleichstabelle: Tabelle 21 ohne Ausschluss der Patientin E1}

Tabelle A10: Als Vergleich zur Tabelle 21 (S. 78) sind hier alle einseitig signifikanten Korrelationen der Scores $\Delta R_{\text {Rechnen }}$ und $\Delta R_{\text {Babyschreien }}$ der Parameter HR, sBP, TPRI, PEP und BRS mit den in Kap. 4.5.2 genannten Variablen aufgeführt - die Berechnungen fanden ohne den Ausschluss der Patientin E1 statt (s. Kap. 4.2.5). So soll geklärt werden, inwiefern der Ausschluss der Patientin E1 Einfluss auf die Ergebnisse hatte.

\begin{tabular}{|c|c|c|c|c|c|}
\hline & \multicolumn{5}{|c|}{$\Delta \mathbf{R}_{\text {Rechnen }}$ (Änderung Recovery) } \\
\hline & $\Delta \mathrm{R}_{H R R e}$ & $\triangle \mathrm{R}_{S B P R e}$ & $\Delta \mathrm{R}_{\text {TPRI Re }}$ & $\Delta \mathrm{R}_{P E P R e}$ & $\Delta \mathrm{R}_{B R S R e}$ \\
\hline $\begin{array}{c}\text { Scl-90-R GSI } \\
\text { (Differenz: Baseline - Follow-up) }\end{array}$ & & & $\begin{array}{l}\mathrm{r}=-0,44 \\
p<0,05\end{array}$ & & \\
\hline $\begin{array}{c}\text { BDI } \\
\text { (Differenz: Baseline - Follow-up) }\end{array}$ & $\begin{array}{l}r=-0,56 \\
p<0,01\end{array}$ & & & & \\
\hline $\begin{array}{c}\text { BPI-Cut-20 } \\
\text { (Differenz: Baseline - Follow-up) }\end{array}$ & & & $\begin{array}{l}r=-0,60 \\
p<0,01\end{array}$ & & \\
\hline $\begin{array}{c}\text { BSL-Gesamtskala } \\
\text { (Differenz: Baseline - Follow-up) }\end{array}$ & $\begin{array}{l}r=-0,42 \\
p<0,05\end{array}$ & & $\begin{array}{l}r=-0,39 \\
p<0,05\end{array}$ & & \\
\hline \multirow[t]{3}{*}{ „Therapiedauer gesamt“ } & & & & $\begin{array}{l}r=-0,37 \\
p<0,05\end{array}$ & \\
\hline & \multicolumn{5}{|c|}{$\Delta \mathbf{R}_{\text {Babyschreien }}$ (Änderung Recovery) } \\
\hline & $\Delta \mathrm{R}_{H R B}$ & $\triangle \mathrm{R}_{S B P B}$ & $\Delta \mathrm{R}_{T P R I B}$ & $\triangle \mathrm{R}_{P E P B}$ & $\triangle \mathrm{R}_{B R S B}$ \\
\hline „Therapiedauer gesamt“ & & & & & $\begin{array}{l}r=-0,42 \\
p<0,05\end{array}$ \\
\hline „Therapiedauer Station 9“ & & & & & $\begin{array}{l}r=-0,40 \\
p<0,05\end{array}$ \\
\hline
\end{tabular}




\subsection{Vergleichstabelle: Tabelle 21 mit Ausschluss der Patientin E2}

Tabelle A11: Als Vergleich zur Tabelle 21 (S. 78) sind hier alle einseitig signifikanten Korrelationen der Scores $\Delta R_{\text {Rechnen }}$ und $\Delta R_{\text {Babyschreien }}$ der Parameter HR, sBP, TPRI, PEP und BRS mit den in Kap. 4.5.2 genannten Variablen aufgeführt - die Berechnungen fanden unter Ausschluss der Patientin E1 und der Patientin E2 statt (s. Kap. 4.2.5). So soll geklärt werden, inwiefern der Einschluss der Patientin E2 Einfluss auf die Ergebnisse hatte.

\begin{tabular}{|c|c|c|c|c|c|}
\hline & \multicolumn{5}{|c|}{$\Delta \mathbf{R}_{\text {Rechnen }}$ (Änderung Recovery) } \\
\hline & $\Delta \mathrm{R}_{H R R e}$ & $\Delta \mathrm{R}_{S B P R e}$ & $\Delta \mathrm{R}_{\text {TPRI Re }}$ & $\Delta \mathrm{R}_{P E P R e}$ & $\Delta \mathrm{R}_{B R S R e}$ \\
\hline $\begin{array}{c}\text { Scl-90-R GSI } \\
\text { (Differenz: Baseline - Follow-up) }\end{array}$ & & & $\begin{array}{l}r=-0,45 \\
p<0,05\end{array}$ & & $\begin{array}{l}r=-0,38 \\
p<0,05\end{array}$ \\
\hline $\begin{array}{c}\text { BDI } \\
\text { (Differenz: Baseline - Follow-up) }\end{array}$ & $\begin{array}{l}r=-0,55 \\
p<0,01\end{array}$ & & & & \\
\hline $\begin{array}{l}\text { IES-R Skala Übererregung } \\
\text { (Differenz Baseline - Follow-up) }\end{array}$ & & & & & $\begin{array}{l}r=-0,42 \\
p<0,05\end{array}$ \\
\hline $\begin{array}{c}\text { BPI-Cut-20 } \\
\text { (Differenz: Baseline - Follow-up) }\end{array}$ & & & $\begin{array}{l}r=-0,66 \\
p<0,01\end{array}$ & & \\
\hline $\begin{array}{c}\text { BSL-Gesamtskala } \\
\text { (Differenz: Baseline - Follow-up) }\end{array}$ & $\begin{array}{l}r=-0,43 \\
p<0,05\end{array}$ & & $\begin{array}{l}r=-0,37 \\
p=0,06\end{array}$ & & \\
\hline \multirow[t]{3}{*}{ „Therapiedauer gesamt“ } & & & & $\begin{array}{l}r=-0,35 \\
p=0,06\end{array}$ & \\
\hline & \multicolumn{5}{|c|}{$\Delta \mathbf{R}_{\text {Babyschreien }}$ (Änderung Recovery) } \\
\hline & $\Delta \mathrm{R}_{H R B}$ & $\triangle \mathrm{R}_{S B P B}$ & $\Delta \mathrm{R}_{T P R I B}$ & $\triangle \mathrm{R}_{P E P B}$ & $\Delta \mathrm{R}_{B R S B}$ \\
\hline „Therapiedauer gesamt“ & & & & & $\begin{aligned} r & =0,34 \\
p & =0,06\end{aligned}$ \\
\hline
\end{tabular}




\subsection{Sensitivitätsanalyse}

Mit dieser Sensitivitätsanalyse soll der Frage nachgegangen werden, welchen Einfluss Änderungen in der Betablockermedikation auf die Ergebnisse gehabt haben könnten. Wie in Kapitel 5.3.1 beschrieben, soll eine Betrachtung der drei Patientinnen erfolgen, bei denen Betablocker zwischen Baseline und Follow-up an- oder abgesetzt wurden.

In Kapitel 4.2.4 wurden Ruhe 1 und Entspannungsmusik gegenübergestellt. Tabelle A13 listet die Ergebnisse der Sensitivitätsanalyse bezüglich dieser „Ruhe“-ANOVA auf, wobei die gleichen Rechnungen unter Ausschluss der drei Patientinnen mit Wechsel in der Betablockermedikation durchgeführt und zusätzlich das partielle Eta-Quadrat bestimmt wurden. In Tabelle A12 findet sich lediglich die Ergänzung der Effektstärken der ursprünglichen ANOVA in Kapitel 4.2.4 ohne Ausschluss der drei Patientinnen. So kann ein direkter Vergleich der Effektstärken erfolgen. Für alle anderen Resultate der Berechnungen ohne Ausschluss sei auf Kapitel 4.2.4 verwiesen.

Tabelle A12: Für einen Vergleich im Zuge der Sensitivitätsanalyse wurde hier die „Ruhe“-ANOVA aus Kap. 4.2 .4 (Tab. 13, S. 55) um die Effektstärke in Form des partiellen Eta-Quadrat ( $\left.\eta^{2}\right)$ ergänzt. Diese aufgeführten Effektstärken beziehen sich somit auf Rechnungen, in denen keine der drei Patientinnen mit Wechsel in der Betablockermedikation ausgeschlossen wurde.

\begin{tabular}{|c|c|c|c|c|c|c|}
\hline \multicolumn{2}{|c|}{} & HR & sBP & TPRI & PEP & BRS \\
\hline Messzeitpunkt & $\begin{array}{c}\text { Partielles Eta- } \\
\text { Quadrat }\left(\eta^{2}\right)\end{array}$ & 0,10 & 0,001 & 0,24 & 0,14 & 0,001 \\
\hline Ruhephase & $\begin{array}{c}\text { Partielles Eta- } \\
\text { Quadrat }\left(\eta^{2}\right)\end{array}$ & 0,47 & 0,11 & 0,39 & 0,26 & 0,24 \\
\hline $\begin{array}{c}\text { Wechselwirkung } \\
\text { Messzeitpunkt } x \\
\text { Ruhephase }\end{array}$ & $\begin{array}{c}\text { Partielles Eta- } \\
\text { Quadrat }\left(\eta^{2}\right)\end{array}$ & 0,04 & 0,00 & 0,01 & 0,06 & 0,02 \\
\hline
\end{tabular}


Tabelle A13 („,Ruhe“-ANOVA, vergleiche Tab. 13, S. 55): Phasenmittelwerte und Standardabweichungen der Phasen Ruhe 1 und Entspannungsmusik (jeweils für Baseline und Follow-up) und eine $2 \times 2$ ANOVA für Messwiederholungen für die Parameter HR in [bpm], sBP in [mmHg], TPRI in $\left[\right.$ dyn $\left.* \mathrm{~s}^{*} \mathrm{~m}^{2} / \mathrm{cm}^{5}\right]$, PEP in [ms] und BRS in [ms $/ \mathrm{mmHg}$-alles im Zuge der Sensitivitätsanalyse (s. zum Vergleich Kap. 4.2.4) und damit unter Ausschluss der drei Patientinnen mit Wechsel in der Betablockermedikation. Angabe der Effektstärke durch das partielle Eta-Quadrat $\left(\eta^{2}\right)$.

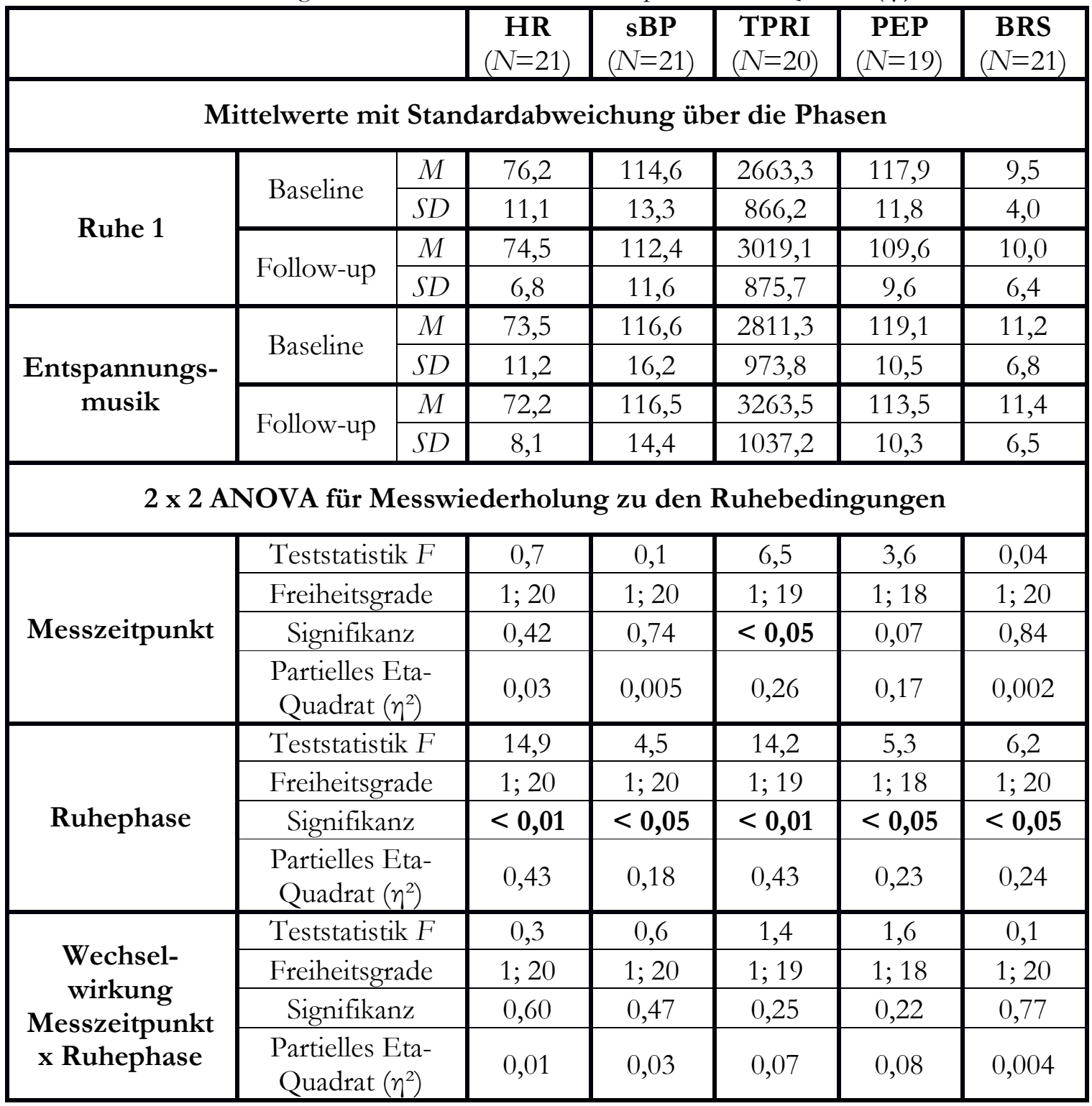


Im Folgenden sind die Ergebnisse aus dem Vergleich der Ruheaktivierung von Baseline und Follow-up aufgeführt. Tabelle A14 gibt nochmals die in Kap.4.3.4 berichteten Resultate der abhängigen t-Tests (Ruhe 1 Baseline vs. Ruhe 1 Follow-up) wieder, hier sind somit weiterhin die drei Patientinnen eingeschlossen. In Tabelle A15 finden sich die Zahlen zu einem Wilcoxon-Vorzeichen-Rang-Test, da die BRS in Ruhe 1 nicht normalverteilt war. Auch hier entsprechen die Zahlen denen in Kapitel 4.3.4, ein Ausschluss der drei Patientinnen mit Änderungen in der Betablockermedikation erfolgte hier nicht. Für die fünf Parameter wurde jeweils die Effektstärke (Cohen's d) ergänzt. In Tabelle A16 und A17 findet sich nun die eigentliche Sensitivitätsanalyse. Die soeben beschriebenen Rechnungen wurden unter Ausschluss der drei Patientinnen widerholt und es wurde wiederum die jeweilige Effektstärke berechnet.

Tabelle A14: Abhängige t-Tests für den Vergleich Ruhe 1 Baseline und Ruhe 1 Follow-up für die Parameter HR in [bpm], sBP in [mmHg], TPRI in $\left[\mathrm{dyn}^{*} \mathrm{~s}^{*} \mathrm{~m}^{2} / \mathrm{cm}^{5}\right]$ und PEP in [ms]. Die hier aufgeführten Ergebnisse entsprechen den Zahlen, die in Kapitel 4.3.4 berichtet werden. Die drei Patientinnen mit Wechsel in der Betablockermedikation sind somit eingeschlossen. Angabe der Effektstärke mittels Cohen's $d$.

\begin{tabular}{|c|c|c|c|c|c|}
\hline \multicolumn{2}{|c|}{ Ruhe 1 } & $\begin{array}{c}\text { HR } \\
\mathbf{N}=25)\end{array}$ & $\begin{array}{c}\text { sBP } \\
(\mathbf{N}=25)\end{array}$ & $\begin{array}{c}\text { TPRI } \\
(\mathbf{N}=25)\end{array}$ & $\begin{array}{c}\text { PEP } \\
(\mathbf{N}=22)\end{array}$ \\
\hline \multirow{2}{*}{ Baseline } & $M$ & 77,8 & 115,0 & 2651,5 & 117,2 \\
\cline { 2 - 6 } & $S D$ & 11,4 & 13,2 & 857,8 & 11,9 \\
\hline \multirow{2}{*}{ Follow-up } & $M$ & 74,2 & 115,8 & 3038,2 & 110,3 \\
\cline { 2 - 6 } & $S D$ & 6,4 & 16,4 & 952,8 & 9,9 \\
\hline \multicolumn{2}{|c|}{ Teststatistik t } & 1,8 & $-0,2$ & $-2,3$ & 1,9 \\
\hline \multicolumn{2}{|c|}{ Freiheitsgrade } & 24 & 24 & 24 & 21 \\
\hline \multicolumn{2}{|c|}{ Signifikanz } & $\begin{array}{c}<\mathbf{0 , 0 5} \\
(\text { einseitig) }\end{array}$ & $\begin{array}{c}0,83 \\
\text { (zweiseitig) }\end{array}$ & $\begin{array}{c}<\mathbf{0 , 0 5} \\
(\text { zweiseitig) }\end{array}$ & $\begin{array}{c}0,08 \\
\text { (zweiseitig) }\end{array}$ \\
\hline \multicolumn{2}{|c|}{ Cohen's $d$} & 0,37 & $-0,05$ & $-0,42$ & 0,63 \\
\hline
\end{tabular}

Tabelle A15: Wilcoxon-Vorzeichen-Rang-Test für den Vergleich Ruhe 1 Baseline und Ruhe 1 Follow-up für den Parameter BRS in [ms $/ \mathrm{mmHg}$. Die hier aufgeführten Ergebnisse entsprechen den Zahlen, die in Kapitel 4.3.4 berichtet werden. Die drei Patientinnen mit Wechsel in der Betablockermedikation sind somit eingeschlossen. Angabe der Effektstärke mittels $\eta^{2}$ und Cohen's $d$.

\begin{tabular}{|c|c|c|c|c|c|c|}
\hline Ruhe 1 & $\begin{array}{c}\text { Baseline } \\
\text { Median }\end{array}$ & $\begin{array}{c}\text { Follow-up } \\
\text { Median }\end{array}$ & $z$ & $\begin{array}{c}\text { Signifikanz } \\
\text { (zweiseitig) }\end{array}$ & $\begin{array}{c}\text { Partielles } \\
\text { Eta-Quadrat }\left(\eta^{2}\right)\end{array}$ & $\begin{array}{c}\text { Cohen's } \\
d\end{array}$ \\
\hline $\begin{array}{c}\text { BRS } \\
(\mathrm{N}=25)\end{array}$ & 9,2 & 8,3 & $-0,5$ & 0,65 & 0,01 & 0,19 \\
\hline
\end{tabular}


Tabelle A16: Abhängige t-Tests für den Vergleich Ruhe 1 Baseline und Ruhe 1 Follow-up für die Parameter HR in [bpm], sBP in [mmHg], TPRI in [dyn $\left.{ }^{*} \mathrm{~m}^{2} / \mathrm{cm}^{5}\right]$ und PEP in [ms], s. auch Kapitel 4.3.4. Die Rechnungen wurden im Zuge der Sensitivitätsanalyse durchgeführt und fanden somit unter Ausschluss der drei Patientinnen mit Wechsel in der Betablockermedikation statt. Angabe der Effektstärke mittels Cohen's $d$.

\begin{tabular}{|c|c|c|c|c|c|}
\hline $\begin{array}{c}\text { Ruhe 1 } \\
\text { (Sensitivitätsanalyse) }\end{array}$ & $\begin{array}{c}\text { HR } \\
(\mathrm{N}=22)\end{array}$ & $\begin{array}{c}\text { sBP } \\
(\mathrm{N}=22)\end{array}$ & $\begin{array}{c}\text { TPRI } \\
(\mathrm{N}=22)\end{array}$ & $\begin{array}{c}\text { PEP } \\
\mathbf{N}=20)\end{array}$ \\
\hline \multirow{2}{*}{ Baseline } & $M$ & 76,4 & 114,9 & 2648,3 & 117,1 \\
\cline { 2 - 6 } & $S D$ & 10,9 & 13,0 & 862,4 & 12,1 \\
\hline \multirow{2}{*}{ Follow-up } & $M$ & 74,6 & 114,0 & 2986,8 & 109,4 \\
\cline { 2 - 6 } & $S D$ & 6,6 & 13,6 & 868,6 & 9,4 \\
\hline Teststatistik t & 1,0 & 0,2 & $-2,0$ & 2,0 \\
\hline Freiheitsgrade & 21 & 21 & 21 & 19 \\
\hline Signifikanz & 0,17 & 0,41 & 0,06 & 0,06 \\
$($ (einseitig) & $($ einseitig) & (zweiseitig) & (zweiseitig) \\
\hline Cohen's $d$ & 0,19 & 0,07 & $-0,39$ & 0,70 \\
\hline
\end{tabular}

Tabelle A17: Wilcoxon-Vorzeichen-Rang-Test für den Vergleich Ruhe 1 Baseline und Ruhe 1 Follow-up für den Parameter BRS in [ms/mmHg], s. auch Kapitel 4.3.4. Die Rechnungen wurden im Zuge der Sensitivitätsanalyse durchgeführt und fanden somit unter Ausschluss der drei Patientinnen mit Wechsel in der Betablockermedikation statt. Angabe der Effektstärke mittels $\eta^{2}$ und Cohen's $d$.

\begin{tabular}{|c|c|c|c|c|c|c|}
\hline $\begin{array}{c}\text { Ruhe 1 } \\
\text { (Sensitivitäts } \\
\text {-analyse) }\end{array}$ & $\begin{array}{c}\text { Baseline } \\
\text { Median }\end{array}$ & $\begin{array}{c}\text { Follow- } \\
\text { up } \\
\text { Median }\end{array}$ & $z$ & $\begin{array}{c}\text { Signifikanz } \\
\text { (zweiseitig) }\end{array}$ & $\begin{array}{c}\text { Partielles } \\
\text { Eta-Quadrat } \\
\left(\eta^{2}\right)\end{array}$ & $\begin{array}{c}\text { Cohen's } \\
d\end{array}$ \\
\hline BRS $(\mathrm{N}=22)$ & 8,8 & 8,3 & $-0,7$ & 0,52 & 0,02 & 0,29 \\
\hline
\end{tabular}

Die Tabellen A18, A19, A22 und A23 präsentieren die Ergebnisse der Sensitivitätsanalyse bezüglich der Reactivity-ANOVAs und der Recovery-ANOVAs. Es wurden die gleichen Rechnungen wie in Kapitel 4.3.5 und 4.3.6 unter Ausschluss der zwei Patientinnen (Patientin E1 ist bereits unabhängig von der Sensitivitätsanalyse ausgeschlossen) vorgenommen und um das partielle Eta-Quadrat als Effektstärke ergänzt. Für die Resultate ohne Ausschluss der zwei Patientinnen sei auf die Kapitel 4.3.5 und Kap. 4.3.6 verwiesen, an dieser Stelle werden lediglich die Effektstärken für die Rechnungen ohne Ausschluss aufgeführt (Tabellen A20, A21, A24 und A25). 
Tabelle A18 (Reactivity Rechnen, s. Tab. 15, S. 70): Phasenmittelwerte und Standardabweichungen der Phasen Ruhe 1 und Rechnen (jeweils für Baseline und Follow-up) und eine 2 x 2 ANOVA für Messwiederholungen für die Parameter HR in [bpm], sBP in [mmHg], TPRI in [dyn $\mathrm{s}^{*} \mathrm{~m}^{2} / \mathrm{cm}^{5}$ ], PEP in [ms] und BRS in [ms $/ \mathrm{mmHg}$ - alles im Zuge der Sensitivitätsanalyse (s. zum Vergleich Kap. 4.3.5) und damit unter Ausschluss der drei Patientinnen mit Wechsel in der Betablockermedikation. Angabe der Effektstärke durch das partielle Eta-Quadrat $\left(\eta^{2}\right)$.

\begin{tabular}{|c|c|c|c|c|c|c|c|}
\hline & & & $\begin{array}{c}\mathbf{H R} \\
(N=22) \\
\end{array}$ & $\begin{array}{c}\mathbf{s B P} \\
(N=22) \\
\end{array}$ & $\begin{array}{c}\text { TPRI } \\
(N=22) \\
\end{array}$ & $\begin{array}{c}\text { PEP } \\
(N=20) \\
\end{array}$ & $\begin{array}{c}\text { BRS } \\
(N=22) \\
\end{array}$ \\
\hline \multicolumn{8}{|c|}{ Mittelwerte mit Standardabweichung über die Phasen } \\
\hline \multirow{4}{*}{ Ruhe 1} & \multirow{2}{*}{ Baseline } & $M$ & 76,4 & 114,9 & 2648,3 & 117,1 & 9,7 \\
\hline & & $S D$ & 10,9 & 13,0 & 862,4 & 12,1 & 4,0 \\
\hline & \multirow{2}{*}{ Follow-up } & $M$ & 74,6 & 114,0 & 2986,8 & 109,4 & 10,0 \\
\hline & & $S D$ & 6,6 & 13,6 & 868,6 & 9,4 & 6,3 \\
\hline \multirow{4}{*}{ Rechnen } & \multirow{2}{*}{ Baseline } & $M$ & 80,3 & 121,1 & 2631,0 & 115,0 & 8,5 \\
\hline & & $S D$ & 11,3 & 12,5 & 959,4 & 11,7 & 3,5 \\
\hline & \multirow{2}{*}{ Follow-up } & $M$ & 77,5 & 120,9 & 2989,5 & 109,6 & 8,8 \\
\hline & & $S D$ & 7,2 & 15,3 & 799,8 & 7,7 & 3,7 \\
\hline \multicolumn{8}{|c|}{$2 \times 2$ ANOVA für Messwiederholung zur Reactivity Rechnen } \\
\hline \multirow{4}{*}{ Messzeitpunkt } & \multicolumn{2}{|c|}{ Teststatistik F } & 1,6 & 0,03 & 4,2 & 3,4 & 0,1 \\
\hline & \multicolumn{2}{|c|}{ Freiheitsgrade } & $1 ; 21$ & $1 ; 21$ & $1 ; 21$ & $1 ; 19$ & $1 ; 21$ \\
\hline & \multicolumn{2}{|c|}{ Signifikanz } & 0,22 & 0,87 & 0,05 & 0,08 & 0,76 \\
\hline & \multicolumn{2}{|c|}{$\begin{array}{c}\text { Partielles Eta- } \\
\text { Quadrat }\left(\eta^{2}\right)\end{array}$} & 0,07 & 0,001 & 0,17 & 0,15 & 0,005 \\
\hline \multirow{4}{*}{$\begin{array}{l}\text { Stressor } \\
\text { Rechnen }\end{array}$} & \multicolumn{2}{|c|}{ Teststatistik F } & 58,5 & 35,9 & 0,03 & 1,1 & 7,7 \\
\hline & \multicolumn{2}{|c|}{ Freiheitsgrade } & 1; 21 & $1 ; 21$ & $1 ; 21$ & $1 ; 19$ & $1 ; 21$ \\
\hline & \multicolumn{2}{|c|}{ Signifikanz } & $<0,001$ & $<0,001$ & 0,86 & 0,30 & $<0,05$ \\
\hline & \multicolumn{2}{|c|}{$\begin{array}{c}\text { Partielles Eta- } \\
\text { Quadrat }\left(\eta^{2}\right)\end{array}$} & 0,74 & 0,63 & 0,002 & 0,06 & 0,27 \\
\hline \multirow{4}{*}{$\begin{array}{l}\text { Wechsel- } \\
\text { wirkung } \\
\text { Messzeitpunkt } \\
\text { x Stressor }\end{array}$} & \multicolumn{2}{|c|}{ Teststatistik F } & 2,7 & 0,1 & 0,1 & 1,7 & 0,001 \\
\hline & \multicolumn{2}{|c|}{ Freiheitsgrade } & $1 ; 21$ & $1 ; 21$ & $1 ; 21$ & $1 ; 19$ & $1 ; 21$ \\
\hline & \multicolumn{2}{|c|}{ Signifikanz } & 0,12 & 0,76 & 0,80 & 0,20 & 0,97 \\
\hline & \multicolumn{2}{|c|}{$\begin{array}{l}\text { Partielles Eta- } \\
\text { Quadrat }\left(\eta^{2}\right)\end{array}$} & 0,11 & 0,005 & 0,003 & 0,08 & 0,00 \\
\hline
\end{tabular}


Tabelle A19 (Reactivity Babyschreien, s. Tab. 16, S. 71): Phasenmittelwerte und Standardabweichungen der Phasen Ruhe 1 und Babyschreien (jeweils für Baseline und Follow-up) und eine 2 x 2 ANOVA für Messwiederholungen für die Parameter HR in [bpm], sBP in [mmHg], TPRI in [dyn* $\mathrm{s}^{*} \mathrm{~m}^{2} / \mathrm{cm}^{5}$ ], PEP in [ms] und BRS in [ms $/ \mathrm{mmHg}$ - alles im Zuge der Sensitivitätsanalyse (s. zum Vergleich Kap. 4.3.5) und damit unter Ausschluss der drei Patientinnen mit Wechsel in der Betablockermedikation. Angabe der Effektstärke durch das partielle Eta-Quadrat $\left(\eta^{2}\right)$.

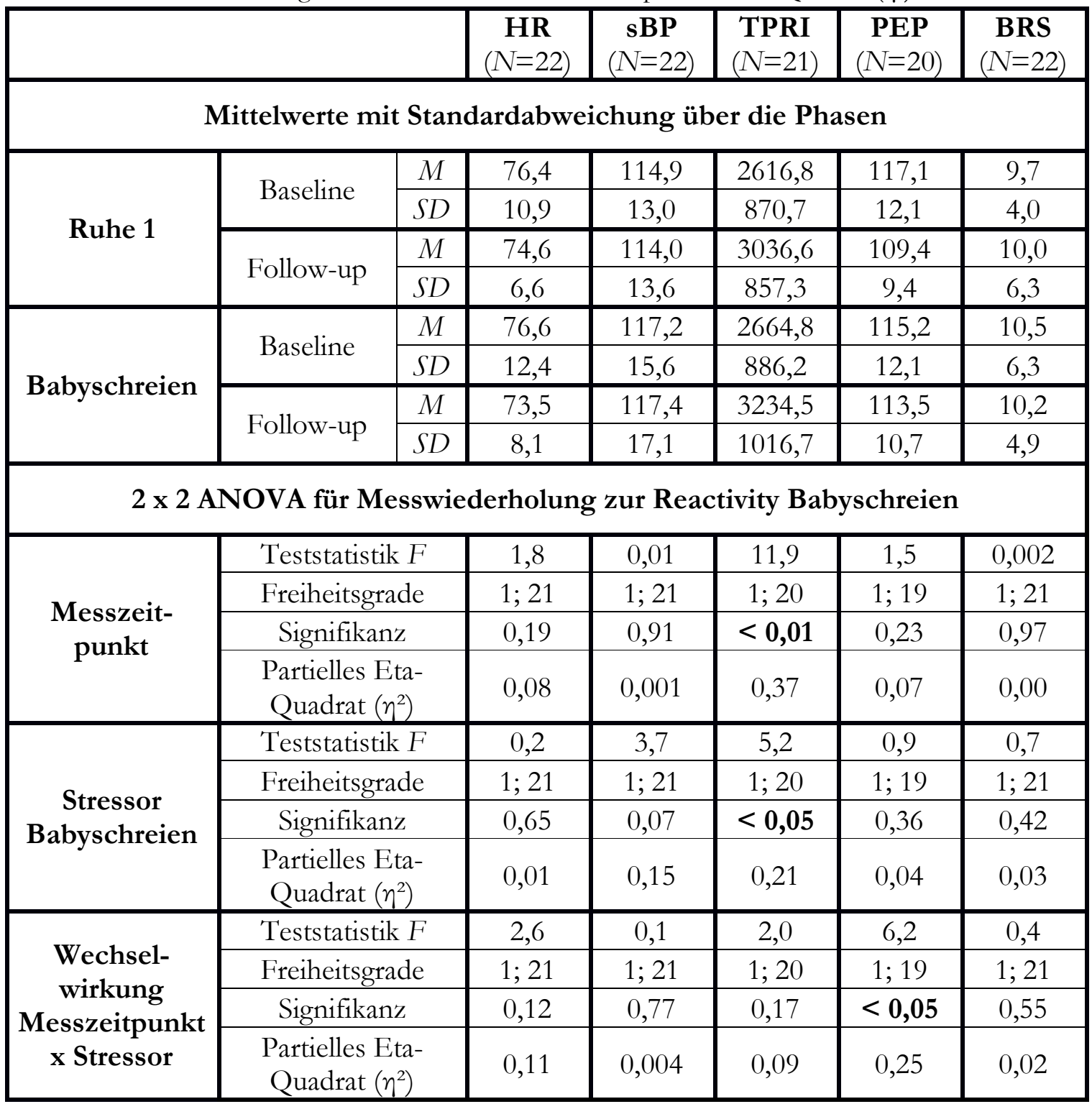


Tabelle A20: Für den Vergleich in der Sensitivitätsanalyse wurde hier die ANOVA „Reactivity Rechnen“ aus Kap. 4.3.5 (Tab. 15, S. 70) um die Effektstärken in Form des partiellen Eta-Quadrat $\left(\eta^{2}\right)$ ergänzt. Die aufgeführten Effektstärken beziehen sich somit auf Rechnungen, in denen lediglich die Patientin E1 und nicht die anderen beiden Patientinnen mit Wechsel in der Betablockermedikation ausgeschlossen wurden.

\begin{tabular}{|c|c|c|c|c|c|c|}
\hline \multicolumn{2}{|c|}{ Reactivity Rechnen } & HR & sBP & TPRI & PEP & BRS \\
\hline Messzeitpunkt & $\begin{array}{c}\text { Partielles Eta- } \\
\text { Quadrat }\left(\eta^{2}\right)\end{array}$ & 0,10 & 0,005 & 0,22 & 0,13 & 0,005 \\
\hline Stressor Rechnen & $\begin{array}{c}\text { Partielles Eta- } \\
\text { Quadrat }\left(\eta^{2}\right)\end{array}$ & 0,68 & 0,58 & 0,01 & 0,04 & 0,24 \\
\hline $\begin{array}{c}\text { Wechselwirkung } \\
\text { Messzeitpunkt x Stressor }\end{array}$ & $\begin{array}{c}\text { Partielles Eta- } \\
\text { Quadrat }\left(\eta^{2}\right)\end{array}$ & 0,06 & 0,00 & 0,00 & 0,05 & 0,00 \\
\hline
\end{tabular}

Tabelle A21: Für den Vergleich in der Sensitivitätsanalyse wurde hier die ANOVA „Reactivity Babyschreien“ aus Kap. 4.3.5 (Tab. 16, S. 71) um die Effektstärken in Form des partiellen EtaQuadrat $\left(\eta^{2}\right)$ ergänzt. Die aufgeführten Effektstärken beziehen sich somit auf Rechnungen, in denen lediglich die Patientin E1 und nicht die anderen beiden Patientinnen mit Wechsel in der Betablockermedikation ausgeschlossen wurden.

\begin{tabular}{|c|c|c|c|c|c|c|}
\hline \multicolumn{2}{|c|}{ Reactivity Babyschreien } & HR & sBP & TPRI & PEP & BRS \\
\hline Messzeitpunkt & $\begin{array}{c}\text { Partielles Eta- } \\
\text { Quadrat }\left(\eta^{2}\right)\end{array}$ & 0,11 & 0,005 & 0,41 & 0,06 & 0,001 \\
\hline Stressor Babyschreien & $\begin{array}{c}\text { Partielles Eta- } \\
\text { Quadrat }\left(\eta^{2}\right)\end{array}$ & 0,02 & 0,11 & 0,21 & 0,06 & 0,04 \\
\hline $\begin{array}{c}\text { Wechselwirkung } \\
\text { Messzeitpunkt x Stressor }\end{array}$ & $\begin{array}{c}\text { Partielles Eta- } \\
\text { Quadrat }\left(\eta^{2}\right)\end{array}$ & 0,05 & 0,001 & 0,04 & 0,20 & 0,01 \\
\hline
\end{tabular}


Tabelle A22 (Recovery Rechnen, Tab. 17, S. 72): Phasenmittelwerte und Standardabweichungen der Phasen Rechnen und Entspannungsmusik (jeweils für Baseline und Follow-up) und eine 2 × 2 ANOVA für Messwiederholungen für die Parameter HR in [bpm], sBP in [mmHg], TPRI in [dyn*s* ${ }^{2} / \mathrm{cm}^{5}$ ], PEP in [ms] und BRS in [ms/mmHg] - alles im Zuge der Sensitivitätsanalyse (s. zum Vergleich Kap. 4.3.6) und damit unter Ausschluss der drei Patientinnen mit Wechsel in der Betablockermedikation. Angabe der Effektstärke durch das partielle Eta-Quadrat $\left(\eta^{2}\right)$.

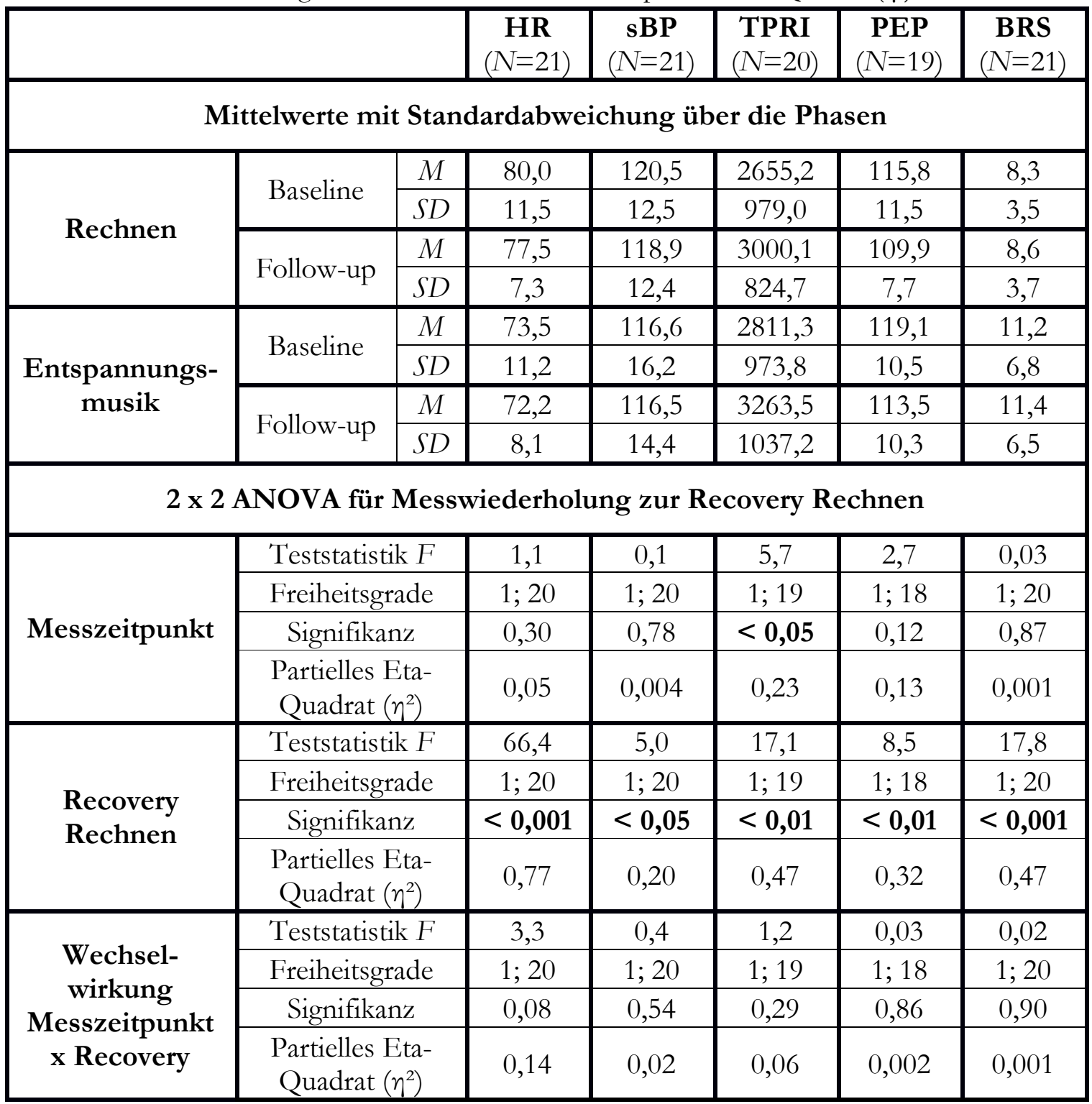


Tabelle A23 (Recovery Babyschreien, Tab. 18, S. 73): Phasenmittelwerte und Standardabweichungen der Phasen Babyschreien und Entspannungsmusik (jeweils für Baseline und Follow-up) und eine $2 \mathrm{x}$ 2 ANOVA für Messwiederholungen für die Parameter HR in [bpm], sBP in [mmHg], TPRI in $\left[\right.$ dyn ${ }^{*} \mathrm{~s}^{*} \mathrm{~m}^{2} / \mathrm{cm}^{5}$ ], PEP in [ms] und BRS in $[\mathrm{ms} / \mathrm{mmHg}$ - alles im Zuge der Sensitivitätsanalyse (s. zum Vergleich Kap. 4.3.6) und damit unter Ausschluss der drei Patientinnen mit Wechsel in der Betablockermedikation. Angabe der Effektstärke durch das partielle Eta-Quadrat $\left(\eta^{2}\right)$.

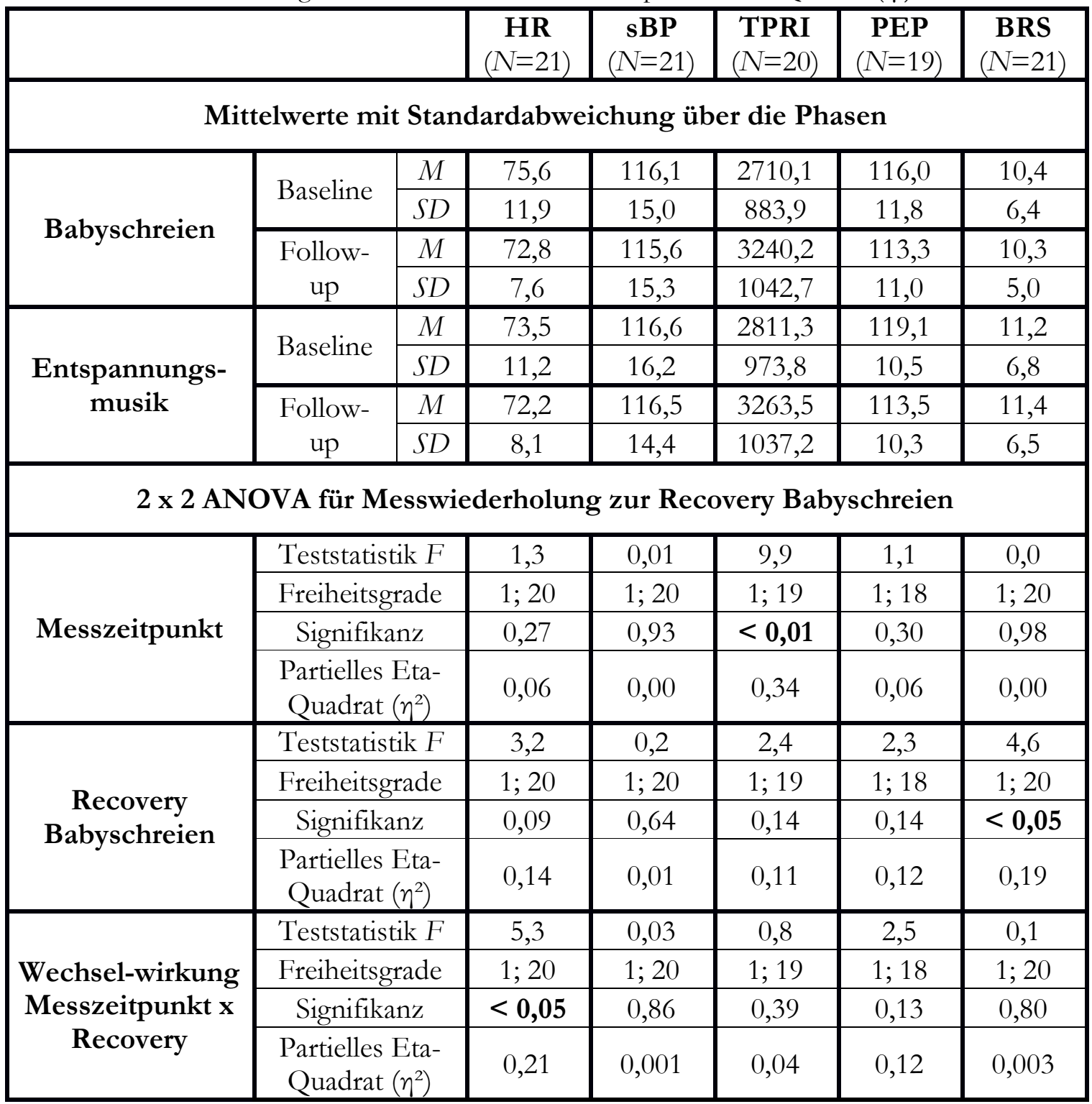


Tabelle A24: Für den Vergleich in der Sensitivitätsanalyse wurde hier die ANOVA „Recovery Rechnen“ aus Kap. 4.3.6 (Tab. 17, S. 72) um die Effektstärken in Form des partiellen Eta-Quadrat $\left(\eta^{2}\right)$ ergänzt. Die aufgeführten Effektstärken beziehen sich somit auf Rechnungen, in denen lediglich die Patientin E1 und nicht die anderen beiden Patientinnen mit Wechsel in der Betablockermedikation ausgeschlossen wurden.

\begin{tabular}{|c|c|c|c|c|c|c|}
\hline \multicolumn{2}{|c|}{ Recovery Rechnen } & HR & sBP & TPRI & PEP & BRS \\
\hline Messzeitpunkt & $\begin{array}{c}\text { Partielles Eta- } \\
\text { Quadrat }\left(\eta^{2}\right)\end{array}$ & 0,09 & 0,001 & 0,27 & 0,12 & 0,001 \\
\hline Recovery Rechnen & $\begin{array}{c}\text { Partielles Eta- } \\
\text { Quadrat }\left(\eta^{2}\right)\end{array}$ & 0,76 & 0,24 & 0,46 & 0,33 & 0,45 \\
\hline $\begin{array}{c}\text { Wechselwirkung } \\
\text { Messzeitpunkt x Recovery }\end{array}$ & $\begin{array}{c}\text { Partielles Eta- } \\
\text { Quadrat }\left(\eta^{2}\right)\end{array}$ & 0,11 & 0,01 & 0,04 & 0,003 & 0,001 \\
\hline
\end{tabular}

Tabelle A25: Für den Vergleich in der Sensitivitätsanalyse wurde hier die ANOVA „Recovery Babyschreien“ aus Kap. 4.3.6 (Tab. 18, S. 73) um die Effektstärken in Form des partiellen EtaQuadrat $\left(\eta^{2}\right)$ ergänzt. Die aufgeführten Effektstärken beziehen sich somit auf Rechnungen, in denen lediglich Patientin E1 und nicht die anderen beiden Patientinnen mit Wechsel in der Betablockermedikation ausgeschlossen wurden.

\begin{tabular}{|c|c|c|c|c|c|c|}
\hline \multicolumn{2}{|c|}{ Recovery Babyschreien } & HR & sBP & TPRI & PEP & BRS \\
\hline Messzeitpunkt & $\begin{array}{c}\text { Partielles Eta- } \\
\text { Quadrat }\left(\eta^{2}\right)\end{array}$ & 0,09 & 0,001 & 0,37 & 0,05 & 0,00 \\
\hline Recovery Babyschreien & $\begin{array}{c}\text { Partielles Eta- } \\
\text { Quadrat }\left(\eta^{2}\right)\end{array}$ & 0,12 & 0,001 & 0,06 & 0,12 & 0,15 \\
\hline $\begin{array}{c}\text { Wechselwirkung } \\
\text { Messzeitpunkt x Recovery }\end{array}$ & $\begin{array}{c}\text { Partielles Eta- } \\
\text { Quadrat }\left(\eta^{2}\right)\end{array}$ & 0,13 & 0,003 & 0,03 & 0,10 & 0,00 \\
\hline
\end{tabular}




\subsection{Allgemeiner Fragebogen}

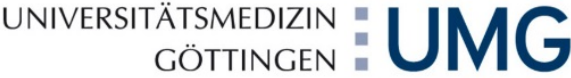

Studie zur physiologischen Stressreagibilität bei Frauen mit komplexer posttraumatischer Belastungsstörung bzw. Borderline-Persönlichkeitsstörung vor und nach psychotherapeutischer Behandlung sowie im Vergleich zu gesunden Frauen

\section{Allgemeiner Fragebogen}

1) Wie alt sind Sie?

2) Wie groß sind Sie?

3) Wieviel wiegen Sie?

4) Befinden Sie sich in einer festen Partnerschaft?
Leben Sie mit Ihrem Partner / Ihrer Partnerin zusammen? Sind sie verheiratet?

5) Sind Sie geschieden?

6) Sind Sie verwitwet?

7) Haben Sie Kinder?

Wenn ja: Welches Alter haben Ihre Kinder?
Code:

Jahre $\mathrm{cm}$ $\mathrm{kg}$

$\square$ Ja $\square$ Nein

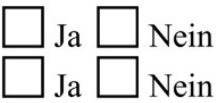

$\square$ Ja $\square$ Nein

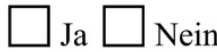

$\square$ Ja $\square$ Nein Jahre

8) Treiben Sie regelmäßig Sport?

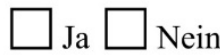

Wieviele Stunden pro Woche? $O \leq 1 / 2 \mathrm{~h} \quad \bigcirc 1 / 2-2 \mathrm{~h} \quad \bigcirc 3-5 \mathrm{~h} \quad \bigcirc 5-10 \mathrm{~h} \quad \bigcirc>10 \mathrm{~h}$

9) Wieviel Alkohol trinken Sie pro Woche?

(Wird vom Untersucher ausgefüllt: $(0,251 \mathrm{Bier} / 0,11$ Wein $/ 2 \mathrm{cl}$ Schnaps $=1$ Drink $)$

10) Rauchen Sie?

Wenn ja: Seit wann?

Wieviele Packungen pro Tag?

(1 Packung $\cong 20$ Zigaretten)

(Wird vom Untersucher ausgefüllt

(Packyears $=$ tägl. konsumierte Zigarettenpackungen $\times$ Jahre Zigarettenkonsum $)$ entspricht dpw)

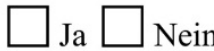

Jahre

Packungen

entspricht Packyears ) 


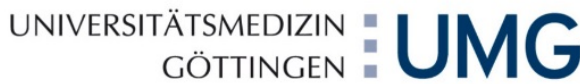

11) Welchen höchsten allgemeinbildenden Schulabschluss haben Sie?

O ohne Schulabschluss

O Hauptschule / Volksschule

O Realschule (Mittlere Reife)

O Polytechnische Oberschule

O Fachhochschule

O Hochschulreife / Abitur (Gymnasium oder EOS)

$\mathrm{O}$ anderer Schulabschluss:

12) Welchen beruflichen Ausbildungsabschluss haben Sie?

(Bitte alle zutreffenden Antworten ankreuzen.)

O ich habe keinen Berufsabschluss und befinde mich nicht in einer Ausbildung

$\mathrm{O}$ ich befinde mich noch in meiner Ausbildung

O beruflich-betriebliche Ausbildung (Lehre)

O beruflich-schulische Ausbildung (Berufsfach- / Handelsschule)

O Fach- / Meister- / Technikerschule / Berufs- / Fachakademie

O Fachhochschule

O Hochschule

$O$ anderer Berufsabschluss:

13) Sind Sie zur Zeit erwerbstätig?

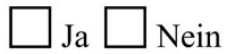

$$
\text { Wenn ja: Wieviele Stunden / Woche? }
$$

O Vollzeit $\geq 35 \mathrm{~h} /$ Woche

O Teilzeit $15 \ldots<35 \mathrm{~h} /$ Woche

O Teilzeit (auch stundenweise) $<15 \mathrm{~h} /$ Woche

14) Nehmen Sie Medikamente? Welche?

(z.B. Pille, Herzmittel,...)

15) Haben Sie Vorerkrankungen?

(z.B. Bluthochdruck, Diabetes, ...)

16) Fühlen Sie sich in letzter Zeit besonders gestresst?

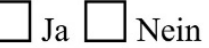




\subsection{Auszug aus dem Fragebogen zu Therapien und Medikation}

Code: [wird vom Forschungszentrum nachgetragen]

Bearbeitungsdatum:

\section{Psychiatrische, psychotherapeutische und stationäre somatische Behandlungen 2009/2010 bis heute}

Sie finden auf den folgenden Seiten eine Tabelle, mit deren Hilfe wir uns einen Überblick über die Behandlungsmaßnahmen verschaffen können, die Sie in den vergangenen Jahren erhalten haben.

Wir benötigen für dieses Forschungsprojekt entsprechende Informationen bezüglich des Zeitraumes von 2009/2010 bis heute (Januar/Februar 2013). Bitte füllen Sie die nachfolgende Tabelle in aller Ruhe und sehr sorgfältig aus. Die gesamte Bearbeitung wird sicherlich einige Zeit benötigen. Vollständige Angaben bezüglich der in Anspruch genommenen Behandlungsmaßnahmen sind allerdings von großer Wichtigkeit, um eine eindeutige Beantwortung der von uns verfolgten Forschungsfragen zu ermöglichen.

\section{Anleitung}

[Im unteren Bereich dieses Blattes ist zum besseren Verständnis der folgenden Erläuterungen exemplarisch ein Ausschnitt der Tabelle dargestellt.]

Sie finden in der angehängten Tabelle für jedes vergangene Jahr ab 2009 eine eigene Zeile, in die Sie bitte die erhaltenen Behandlungsmaßnahmen im jeweiligen Jahr eintragen.

Beim Ausfüllen der Tabelle bitten wir Sie,

1. stationäre psychotherapeutische / psychiatrische Behandlungen (Spalte A),

2. ambulante psychotherapeutische Behandlungen (Spalte B) sowie

3. stationäre körperliche / somatische Behandlungen (Spalte C)

von einander zu unterscheiden und in die jeweiligen Kästchen zu notieren. Bitte verwenden Sie ein Beiblatt oder beschreiben Sie die Rückseite, wenn der vorgegeben Platz nicht ausreicht.

Beispiel:

\begin{tabular}{|l|l|l|l|}
\hline & \multicolumn{1}{|c|}{$\begin{array}{c}\text { Spalte A } \\
\text { Stationäre psychotherapeutische / } \\
\text { psychiatrische Behandlung }\end{array}$} & $\begin{array}{c}\text { Spalte B } \\
\text { Ambulante psychotherapeutische / } \\
\text { psychiatrische Behandlung }\end{array}$ & \multicolumn{1}{c|}{$\begin{array}{c}\text { Spalte C } \\
\text { Stationäre somatische } \\
\text { Behandlung }\end{array}$} \\
\hline 2009 & $\begin{array}{l}\text { 10. April - 12. Mai (ca. 32 Tage): } \\
\text { Station 9 in Göttingen } \\
\text { 20. - 28. August (ca. 9 Tage): } \\
\text { Akutpychiatrie Neudorf }\end{array}$ & $\begin{array}{l}\text { Juni-Dezember: } \\
\text { 1x wöchentlich tiefenpsycholo- } \\
\text { gisch-fundierte Einzeltherapie }\end{array}$ & $\begin{array}{l}\text { O1. - 10. Dezember } \\
\text { (ca. 11 Tage) } \\
\text { Blinddarm-OP }\end{array}$ \\
\hline 2010 & & & \\
\hline$\ldots$ & & & \\
\hline
\end{tabular}




\begin{tabular}{|l|l|l|l|}
\hline & \multicolumn{1}{|c|}{$\begin{array}{c}\text { Stationäre } \\
\text { psychotherapeutische / } \\
\text { Psychiatrische Behandlung }\end{array}$} & $\begin{array}{c}\text { Ambulante } \\
\text { psychotherapeutische / } \\
\text { psychiatrische Behandlung }\end{array}$ & \multicolumn{1}{c|}{$\begin{array}{c}\text { Stationäre somatische } \\
\text { Behandlung }\end{array}$} \\
\hline Beispiel: 2008 & $\begin{array}{l}\text { 10. April - 12. Mai (ca. 32 Tage): } \\
\text { Station 9 in Göttingen; 20. - 28. } \\
\text { August (ca. 9 Tage): Akut- } \\
\text { Psychiatrie Neudorf }\end{array}$ & $\begin{array}{l}\text { Juni - Dezember: ca. 1x pro } \\
\text { Woche tiefenpsychologisch- } \\
\text { fundierte Einzeltherapie; } \\
\text { außerdem ca. alle drei Monate } \\
\text { psychiatrische Behandlung wg. } \\
\text { Medikamenten }\end{array}$ & $\begin{array}{l}\text { 01. - 10. Dezember (ca. 11 Tage) } \\
\text { Blinddarm-OP }\end{array}$ \\
\hline 2009 & & & \\
\hline 2010 & & & \\
\hline
\end{tabular}

Bitte alle Medikamente und die jeweilige Dosierung ganz genau aufschreiben.

\begin{tabular}{|l|l|l|l|l|}
\hline & morgens & mittags & abends & $\begin{array}{l}\text { zur } \\
\text { Nacht }\end{array}$ \\
\hline $\begin{array}{l}\text { Medikament } \\
\text { (Cipramil) }\end{array}$ & $20 \mathrm{mg}$ & $0 \mathrm{mg}$ & $0 \mathrm{mg}$ & $0 \mathrm{mg}$ \\
\hline & & & & \\
\hline & & & & \\
\hline & & & & \\
\hline & & & & \\
\hline & & & & \\
\hline & & & & \\
\hline & & & & \\
\hline & & & & \\
\hline & & & & \\
\hline & & & & \\
\hline
\end{tabular}




\section{Literaturverzeichnis}

Acarturk C, Konuk E, Cetinkaya M, Senay I, Sijbrandij M, Cuijpers P, Aker T (2015): EMDR for Syrian refugees with posttraumatic stress disorder symptoms: results of a pilot randomized controlled trial. Eur J Psychotraumatol $\underline{6}, 27414$

Ackermann S, Hartmann F, Papassotiropoulos A, de Quervain DJ, Rasch B (2013): Associations between basal cortisol levels and memory retrieval in healthy young individuals. J Cogn Neurosci 25, 1896-1907

Albrecht J: Psychophysiologische Stressreagibilität bei Frauen mit posttraumatischer Belastungsstörung (PTBS) sowie der Einfluss einer ausgeprägten Borderline-Symptomatik. Med. Diss. Göttingen 2013

Allen MT, Crowell MD (1989): Patterns of autonomic response during laboratory stressors. Psychophysiology 26, 603-614

American Psychiatric Association: Diagnostic and statistical manual of mental disorders: DSM-IIIR. 3. Auflage; American Psychiatric Association, Washington, DC 1987

American Psychiatric Association: Diagnostic and statistical manual of mental disorders: DSM-IV. 4. Auflage; American Psychiatric Association, Washington, DC 1994

American Psychiatric Association: Diagnostic and statistical manual of mental disorders: DSM-V. 5. Auflage; American Psychiatric Association, Arlington, VA 2013

American Psychiatric Association: Diagnostisches und statistisches Manual psychischer Störungen DSM-5. Hrsg. v. Falkai P, Wittchen H-U, 1. Auflage; Hogrefe, Göttingen 2015

Arditi-Babchuk H, Feldman R, Gilboa-Schechtman E (2009): Parasympathetic reactivity to recalled traumatic and pleasant events in trauma-exposed individuals. J Trauma Stress 22, 254-257

Bacon SL, Keller AJ, Lavoie KL, Campbell TS (2010): Comparison of a three-quarter electrode band configuration with a full electrode band configuration for impedance cardiography. Psychophysiology 47, 1087-1093

Baker DG, West SA, Nicholson WE, Ekhator NN, Kasckow JW, Hill KK, Bruce AB, Orth DN, Geracioti TD (1999): Serial CSF corticotropin-releasing hormone levels and adrenocortical activity in combat veterans with posttraumatic stress disorder. Am J Psychiatry 156, 585588

Ball JS, Links PS (2009): Borderline personality disorder and childhood trauma: evidence for a causal relationship. Curr Psychiatry Rep 11, 63-68

Barnes VA, Johnson MH, Treiber FA (2004): Temporal stability of twenty-four-hour ambulatory hemodynamic bioimpedance measures in African American adolescents. Blood Press Monit $\underline{9}, 173-177$

Beck AT, Ward CH, Mendelson M, Mock J, Erbaugh J (1961): An inventory for measuring depression. Arch Gen Psychiatry $\underline{4}, 561-571$

Beckham JC, Vrana SR, Barefoot JC, Feldman ME, Fairbank J, Moore SD (2002): Magnitude and duration of cardiovascular responses to anger in vietnam veterans with and without posttraumatic stress disorder. J Consult Clin Psychol 70, 228-234

Bernstein EM, Putnam FW (1986): Development, reliability, and validity of a dissociation scale. J Nerv Ment Dis $174,727-735$ 
Berntson GG, Cacioppo JT, Binkley PF, Uchino BN, Quigley KS, Fieldstone A (1994): Autonomic cardiac control. III. Psychological stress and cardiac response in autonomic space as revealed by pharmacological blockades. Psychophysiology $\underline{31}, 599-608$

Blanchard EB, Hickling EJ, Buckley TC, Taylor AE, Vollmer A, Loos WR (1996): Psychophysiology of posttraumatic stress disorder related to motor vehicle accidents: Replication and extension. J Consult Clin Psychol 64, 742-751

Blanchard EB, Hickling EJ, Veazey CH, Buckley TC, Freidenberg BM, Walsh JD, Keefer L (2002): Treatment-related changes in cardiovascular reactivity to trauma cues in motor vehicle accident-related PTSD. Behav Ther $\underline{33}$, 417-426

Blechert J, Michael T, Grossman P, Lajtman M, Wilhelm FH (2007): Autonomic and respiratory characteristics of posttraumatic stress disorder and panic disorder. Psychosom Med $\underline{69}$, 935-943

Bohus M, Limberger MF, Frank U, Sender I, Gratwohl T, Stieglitz RD (2001): Entwicklung der Borderline-Symptom-Liste. Psychother Psychosom Med Psychol 151, 201-211

Bohus M, Limberger MF, Frank U, Chapman AL, Kühler T, Stieglitz RD (2007): Psychometric properties of the Borderline Symptom List (BSL). Psychopathology 40, 126-132

Bohus M, Dyer AS, Priebe K, Krüger A, Kleindienst N, Schmahl C, Niedtfeld I, Steil R (2013): Dialectical behaviour therapy for post-traumatic stress disorder after childhood sexual abuse in patients with and without borderline personality disorder: A randomised controlled trial. Psychother Psychosom 2, 221-233

Borderline Research Unit ZI-Mannheim. https://www.zimannheim.de/fileadmin/user_upload/downloads/forschung/PSM_downloads/BSL_95_ deutsch_Prozentrangnormierungstabellen_09_2004.pdf; Zugriff am 12.10.2017

Borderline Research Unit ZI-Mannheim. https://www.zimannheim.de/fileadmin/user_upload/downloads/forschung/PSM_downloads/BSL_95_ deutsch_Auswertungshinweise_04_2009.pdf; Zugriff am 12.10.2017

Bornschein G: Vergleich der physiologischen Stressreagibilität von Frauen mit komplexen Traumafolgestörungen und gesunden Frauen. Med. Diss. Göttingen 2014

Boscarino JA (2008): A prospective study of PTSD and early-age heart disease mortality among Vietnam veterans: implications for surveillance and prevention. Psychosom Med $\underline{70}, 668$ 676

Boudewyns PA, Hyer L (1990): Physiological response to combat memories and preliminary treatment outcome in Vietnam veteran PTSD patients treated with direct therapeutic exposure. Behav Ther 21, 63-87

Bremner JD, Licinio J, Darnell A, Krystal JH, Owens MJ, Southwick SM, Nemeroff CB, Charney DS (1997): Elevated CSF corticotropin-releasing factor concentrations in posttraumatic stress disorder. Am J Psychiatry 154, 624-629

Briant LJ, Charkoudian N, Hart EC (2016): Sympathetic regulation of blood pressure in normotension and hypertension: when sex matters. Exp Physiol 101, 219-229

Brosius F: SPSS 19. mitp, Heidelberg 2011

Bryant RA, Harvey AG, Guthrie RM, Moulds ML (2003): Acute psychophysiological arousal and posttraumatic stress disorder: a two-year prospective study. J Trauma Stress 16, 439-443 
Buckley TC, Kaloupek DG (2001): A meta-analytic examination of basal cardiovascular activity in posttraumatic stress disorder. Psychosom Med $\underline{63}, 585-594$

Burleson MH, Poehlmann KM, Hawkley LC, Ernst JM, Berntson GG, Malarkey WB, KiecoltGlaser JK, Glaser R, Cacioppo JT (2003): Neuroendocrine and cardiovascular reactivity to stress in mid-aged and older women: Long-term temporal consistency of individual differences. Psychophysiology 40, 358-369

Burns JW, Ferguson ML, Fernquist SK, Katkin ES (1992): Test-retest reliability of inotropic and chronotropic measures of cardiac reactivity. Int J Psychophysiol 12, 165-168

Bylsma LM, Salomon K, Taylor-Clift A, Morris BH, Rottenberg J (2014): RSA reactivity in current and remitted major depressive disorder. Psychosom Med $\underline{76}$, 66-73

Cannon W (1914): The emergency function of the adrenal medulla in pain and the major emotions. Am J Physiol $\underline{33}, 356-372$

Carleton RN, Mulvogue MK, Duranceau S (2015): PTSD personality subtypes in women exposed to intimate-partner violence. Psychol Trauma $\underline{7}, 154-161$

Casanova GM, Domanic J, McCanne TR, Milner JS (1994): Physiological responses to child stimuli in mothers with and without a childhood history of physical abuse. Child Abuse Negl $\underline{18}$, 995-1004

Castillo DT, Joseph JS, Tharp AT, C'de Baca J, Torres-Sena LM, Qualls C, Miller MW (2014): Externalizing and internalizing subtypes of posttraumatic psychopathology and anger expression. J Trauma Stress $\underline{27}, 108-111$

Chang HA, Chang CC, Tzeng NS, Kuo TB, Lu RB, Huang SY (2013): Decreased cardiac vagal control in drug-naïve patients with posttraumatic stress disorder. Psychiatry Investig $\underline{10}$, $121-130$

Chen L, Zhang G, Hu M, Liang X (2015): Eye movement desensitization and reprocessing versus cognitive-behavioral therapy for adult posttraumatic stress disorder: Systematic review and meta-analysis. J Nerv Ment Dis $\underline{203}$, 443-451

Chumaeva N, Hintsanen M, Hintsa T, Ravaja N, Juonala M, Raitakari OT, Keltikangas-Järvinen L (2010): Early atherosclerosis and cardiac autonomic responses to mental stress: a population-based study of the moderating influence of impaired endothelial function. BMC Cardiovasc Disord $\underline{10}, 16$

CNSystems Medizintechnik AG: Task Force ${ }^{\circledR}$ Monitor Benutzerhandbuch V2.2. Eigendruck, Graz 2010

Cohen H, Kotler M, Matar MA, Kaplan Z, Loewenthal U, Miodownik H, Cassuto Y (1998): Analysis of heart rate variability in posttraumatic stress disorder patients in response to a trauma-related reminder. Biol Psychiatry $\underline{44}$, 1054-1059

Cohen H, Benjamin J, Geva AB, Matar MA, Kaplan Z, Kotler M (2000): Autonomic dysregulation in panic disorder and in post-traumatic stress disorder: application of power spectrum analysis of heart rate variability at rest and in response to recollection of trauma or panic attacks. Psychiatry Res $\underline{96}, 1-13$

Cole CR, Blackstone EH, Pashkow FJ, Snader CE, Lauer MS (1999): Heart-rate recovery immediately after exercise as a predictor of mortality. N Engl J Med $\underline{341}, 1351-1357$

Creamer M, Bell R, Failla S (2003): Psychometric properties of the Impact of Event Scale - Revised. Behav Res Ther 41, 1489-1496 
De Bellis MD, Zisk A (2014): The biological effects of childhood trauma. Child Adolesc Psychiatr Clin N Am $\underline{23}, 185-222$

Derogatis LR, Lipman RS, Covi L (1973): SCL-90: an outpatient psychiatric rating scale-preliminary report. Psychopharmacol Bull $\underline{9}, 13-28$

DGPPN, BÄK, KBV, AWMF (Hrsg.) für die Leitliniengruppe Unipolare Depression. S3 Leitlinie/Nationale VersorgungsLeitlinie Unipolare Depression - Langfassung, 2. Auflage. Version 5. 2015. DOI: 10.6101/AZQ/000364. www.depression.versorgungsleitlinien.de; Zugriff am 13.10.2017

Di Rienzo M, Parati G, Castiglioni P, Tordi R, Mancia G, Pedotti A (2001): Baroreflex effectiveness index: an additional measure of baroreflex control of heart rate in daily life. Am J Physiol Regul Integr Comp Physiol 280, R744-751

Diamant M, Harms MP, Immink RV, Van Lieshout JJ, Van Montfrans GA (2002): Twenty-fourhour non-invasive monitoring of systemic haemodynamics and cerebral blood flow velocity in healthy humans. Acta Physiol Scand 175, 1-9

Ebner-Priemer UW, Badeck S, Beckmann C, Wagner A, Feige B, Weiss I, Lieb K, Bohus M (2005): Affective dysregulation and dissociative experience in female patients with borderline personality disorder: a startle response study. J Psychiatr Res $\underline{39}$, 85-92

Edmondson D, Cohen BE (2013): Posttraumatic stress disorder and cardiovascular disease. Prog Cardiovasc Dis $\underline{55}, 548-556$

Edmondson D, Richardson S, Falzon L, Davidson KW, Mills MA, Neria Y (2012): Posttraumatic stress disorder prevalence and risk of recurrence in acute coronary syndrome patients: a meta-analytic review. PloS One 7 , e38915

Edmondson D, Kronish IM, Shaffer JA, Falzon L, Burg MM (2013): Posttraumatic stress disorder and risk for coronary heart disease: a meta-analytic review. Am Heart J 166, 806-814

Elzinga BM, Schmahl CG, Vermetten E, van Dyck R, Bremner JD (2003): Higher cortisol levels following exposure to traumatic reminders in abuse-related PTSD. Neuropsychopharmacology $\underline{28}, 1656-1665$

Fiedler P: Dissoziative Störungen und Konversion: Trauma und Traumabehandlung. 2. Auflage; Beltz, Weinheim 2001

Flatten G, Gast U, Hofmann A, Liebermann P, Reddemann L, Siol T, Wöller W, Petzold ER: Posttraumatische Belastungsstörung: Leitlinie und Quellentext. 2. Auflage; Schattauer, Stuttgart 2004

Flatten G, Gast U, Hofmann A, Knaevelsrud C, Lampe A, Liebermann P, Maercker A, Reddemann L, Wöller W: Posttraumatische Belastungsstörung : S3-Leitlinie und Quellentexte. Schattauer, Stuttgart 2013

Forbes D, Elhai JD, Miller MW, Creamer M (2010): Internalizing and externalizing classes in posttraumatic stress disorder: a latent class analysis. J Trauma Stress $\underline{23}, 340-349$

Forcier K, Stroud LR, Papandonatos GD, Hitsman B, Reiches M, Krishnamoorthy J, Niaura R (2006): Links between physical fitness and cardiovascular reactivity and recovery to psychological stressors: A meta-analysis. Health Psychol $\underline{25}$, 723-39

Fortin J: Continuous non-invasive measurements of cardiovascular function. Tech. Diss. Graz 2007 
Fortin J, Habenbacher W, Gruellenberger R, Wach P, Skrabal F (1998): Real-time monitor for hemodynamic beat-to-beat parameters and power spectra analysis of the biosignals. Proceedings of the 20th Annual International Conference of the IEEE Engineering in Medicine and Biology Society 20 (1), 360-363

Fortin J, Haitchi G, Bojic A, Habenbacher W, Grüllenberger R, Heller A, Pacher R, Wach P, Skrabal F (2001): Validation and verification of the Tasc Force Monitor. Result of clinical studies for FDA 510(k) no. K014063, 1-7

Fortin J, Marte W, Grüllenberger R, Hacker A, Habenbacher W, Heller A, Wagner C, Wach P, Skrabal F (2006a): Continuous non-invasive blood pressure monitoring using concentrically interlocking control loops. Comput Biol Med $\underline{36}$, 941-957

Fortin J, Habenbacher W, Heller A, Hacker A, Grüllenberger R, Innerhofer J, Passath H, Wagner C, Haitchi G, Flotzinger D, et al. (2006b): Non-invasive beat-to-beat cardiac output monitoring by an improved method of transthoracic bioimpedance measurement. Comput Biol Med $\underline{36}, 1185-1203$

Fortin J, Wellisch A, Maier K (2013): CNAP - evolution of continuous non-invasive arterial blood pressure monitoring. Biomed Tech (Berl) $\underline{58}$ (Suppl. 1)

Franke GH: SCL-90-R. Symptom-Checkliste von L.R. Derogatis - deutsche Version: Manual. 2. Auflage; Beltz Test, Göttingen 2000

Franke GH (2001): Möglichkeiten und Grenzen im Einsatz der Symptom-Checkliste SCL-90-R. Verhal Psychosoz Prax $\underline{33}, 475-485$

Franke GH: Symptom-Checklist-90-Standard: SCL-90-S - Manual. Hogrefe, Göttingen 2014

Freyberger HJ, Spitzer C (2005): Dissoziative Störungen. Nervenarzt 76, 893-899

Freyberger HJ, Spitzer C, Stieglitz RD, Kuhn G, Magdeburg N, Bernstein-Carlson E (1998): Fragebogen zu dissoziativen Symptomen (FDS) - Deutsche Adaptation, Reliabilität und Validität der amerikanischen Dissociative Experience Scale (DES). Psychother Psychosom Med Psychol 48, 223-229

Friedman MJ (2013): Finalizing PTSD in DSM-5: getting here from there and where to go next. J Trauma Stress 26, 548-556

Gabbard GO (2005): Mind, brain, and personality disorders. Am J Psychiatry 162, 648-655

Gallagher MW, Resick PA (2012): Mechanisms of change in cognitive processing therapy and prolonged exposure therapy for PTSD: Preliminary evidence for the differential effects of hopelessness and habituation. Cogn Ther Res $\underline{36}$, 750-755

Geracioti TD, Baker DG, Ekhator NN, West SA, Hill KK, Bruce AB, Schmidt D, Rounds-Kugler B, Yehuda R, Keck PE, et al. (2001): CSF norepinephrine concentrations in posttraumatic stress disorder. Am J Psychiatry $\underline{158}, 1227-1230$

Gilbertson MW, Shenton ME, Ciszewski A, Kasai K, Lasko NB, Orr SP, Pitman RK (2002): Smaller hippocampal volume predicts pathologic vulnerability to psychological trauma. Nat Neurosci $\underline{5}, 1242-1247$

Gillin JL, Mills PJ, Nelesen RA, Dillon E, Ziegler MG, Dimsdale JE (1996): Race and sex differences in cardiovascular recovery from acute stress. Int J Psychophysiol 23, 83-90

Gratze G, Fortin J, Holler A, Grasenick K, Pfurtscheller G, Wach P, Schönegger J, Kotanko P, Skrabal F (1998): A software package for non-invasive, real-time beat-to-beat monitoring 
of stroke volume, blood pressure, total peripheral resistance and for assessment of autonomic function. Comput Biol Med 28, 121-142

Griffin MG, Nishith P, Resick PA, Yehuda R (1997): Integrating objective indicators of treatment outcome in posttraumatic stress disorder. Ann N Y Acad Sci $\underline{821}$, 388-409

Griffin MG, Resick PA, Galovski TE (2012): Does physiologic response to loud tones change following cognitive-behavioral treatment for posttraumatic stress disorder? J Trauma Stress $\underline{25}, 25-32$

Gunderson JG, Kolb JE, Austin V (1981): The diagnostic interview for borderline patients. Am J Psychiatry 138, 896-903

Gurvits TV, Metzger LJ, Lasko NB, Cannistraro PA, Tarhan AS, Gilbertson MW, Orr SP, Charbonneau AM, Wedig MM, Pitman RK (2006): Subtle neurologic compromise as a vulnerability factor for combat-related posttraumatic stress disorder: results of a twin study. Arch Gen Psychiatry 63, 571-576

Hamer M, Steptoe A (2007): Association between physical fitness, parasympathetic control, and proinflammatory responses to mental stress. Psychosom Med $\underline{69}$, 660-666

Hauschildt M, Peters MJ, Moritz S, Jelinek L (2011): Heart rate variability in response to affective scenes in posttraumatic stress disorder. Biol Psychol $\underline{88}, 215-222$

Hautzinger M, Bailer M, Worall H, Keller F: Beck-Depressions-Inventar (BDI). Verlag Hans Huber, Bern 1994

Hawk LW, Dougall AL, Ursano RJ, Baum A (2000): Urinary catecholamines and cortisol in recentonset posttraumatic stress disorder after motor vehicle accidents. Psychosom Med $\underline{62}, 423$ 434

Heponiemi T, Elovainio M, Pulkki L, Puttonen S, Raitakari O, Keltikangas-Järvinen L (2007): Cardiac autonomic reactivity and recovery in predicting carotid atherosclerosis: The cardiovascular risk in young finns study. Health Psychol 26, 13-21

Herbold W, Sachsse U (2008): Schritt für Schritt: Differenzialindikation und spezialisierte stationäre Psychotherapie in einer psychiatrisch-psychotherapeutischen Fachklinik der Regionalversorgung. Fortschritte Neurol Psychiatr 76, 155-159

Herman JL (1992): Complex PTSD: A syndrome in survivors of prolonged and repeated trauma. J Trauma Stress $\underline{5}, 377-391$

Herman JL: Die Narben der Gewalt: traumatische Erfahrungen verstehen und überwinden. Kindler, München 1993

Herrmann-Lingen C (2011): Psychosomatik der Herzinsuffizienz - Alles nur Depression? Herz $\underline{36}$, $135-140$

Hopper JW, Spinazzola J, Simpson WB, van der Kolk BA (2006): Preliminary evidence of parasympathetic influence on basal heart rate in posttraumatic stress disorder. J Psychosom Res $\underline{60}, 83-90$

Horowitz M, Wilner N, Alvarez W (1979): Impact of Event Scale: a measure of subjective stress. Psychosom Med 41, 209-218

Hu MX, Lamers F, de Geus EJ, Penninx BW (2016): Differential autonomic nervous system reactivity in depression and anxiety during stress depending on type of stressor. Psychosom Med $\underline{78}, 562-572$ 
Hughes JW, Feldman ME, Beckham JC (2006): Posttraumatic stress disorder is associated with attenuated baroreceptor sensitivity among female, but not male, smokers. Biol Psychol 71, 296-302

Hughes JW, Dennis MF, Beckham JC (2007): Baroreceptor sensitivity at rest and during stress in women with posttraumatic stress disorder or major depressive disorder. J Trauma Stress $\underline{20}, 667-676$

Hüther G (1996): The central adaptation syndrome: psychosocial stress as a trigger for adaptive modifications of brain structure and brain function. Prog Neurobiol 48, 569-612

Hüther G, Sachsse U (2007): Angst- und stressbedingte Störungen. Psychotherapeut 52, 166-179

Ironson G, Taylor CB, Boltwood M, Bartzokis T, Dennis C, Chesney M, Spitzer S, Segall GM (1992): Effects of anger on left ventricular ejection fraction in coronary artery disease. Am J Cardiol $\underline{70}, 281-285$

Joyner MJ, Dietz NM (2003): Sympathetic vasodilation in human muscle. Acta Physiol Scand 177 , 329-336

Joyner MJ, Wallin BG, Charkoudian N (2016): Sex differences and blood pressure regulation in humans. Exp Physiol 101, 349-355

Kapfhammer HP (2014): Trauma- und stressorbezogene Störungen - Diagnostische Konzeptionalisierung im DSM-5. Nervenarzt $\underline{85}$, 553-563

Karl A, Malta LS, Alexander J, Blanchard EB (2004): Startle responses in motor vehicle accident survivors: a pilot study. Appl Psychophysiol Biofeedback 29, 223-231

Karl A, Schaefer M, Malta LS, Dörfel D, Rohleder N, Werner A (2006): A meta-analysis of structural brain abnormalities in PTSD. Neurosci Biobehav Rev $\underline{30}, 1004-1031$

Kasprowicz AL, Manuck SB, Malkoff SB, Krantz DS (1990): Individual differences in behaviorally evoked cardiovascular response: Temporal stability and hemodynamic patterning. Psychophysiology 27, 605-619

Keane TM, Kolb LC, Kaloupek DG, Orr SP, Blanchard EB, Thomas RG, Hsieh FY, Lavori PW (1998): Utility of psychophysiological measurement in the diagnosis of posttraumatic stress disorder: Results from a department of veterans affairs cooperative study. J Consult Clin Psychol 66, 914-923

Keary TA, Hughes JW, Palmieri PA (2009): Women with posttraumatic stress disorder have larger decreases in heart rate variability during stress tasks. Int J Psychophysiol 73, 257-264

Kelsey RM, Blascovich J, Tomaka J, Leitten CL, Schneider TR, Wiens S (1999): Cardiovascular reactivity and adaptation to recurrent psychological stress: Effects of prior task exposure. Psychophysiology $\underline{36}, 818-831$

Kelsey RM, Ornduff SR, Alpert BS (2007): Reliability of cardiovascular reactivity to stress: Internal consistency. Psychophysiology 44, 216-225

Kernberg OF (1967): Borderline personality organization. J Am Psychoanal Assoc 15, 641-685

Kernberg OF: Schwere Persönlichkeitsstörungen: Theorie, Diagnose, Behandlungsstrategien. KlettCotta, Stuttgart 1988

Kibler JL, Ma M (2004): Depressive symptoms and cardiovascular reactivity to laboratory behavioral stress. Int J Behav Med 11, 81-87 
Kibler JL, Lyons JA (2004): Perceived coping ability mediates the relationship between PTSD severity and heart rate recovery in veterans. J Trauma Stress $\underline{17}, 23-29$

Klosterman Rielage J, Hoyt T, Renshaw K (2010): Internalizing and externalizing personality styles and psychopathology in OEF-OIF veterans. J Trauma Stress $\underline{23}$, 350-357

Koelsch S, Jäncke L (2015): Music and the heart. Eur Heart J 36, 3043-3049

Koopman C, Carrion V, Butler LD, Sudhakar S, Palmer L, Steiner H (2004): Relationships of dissociation and childhood abuse and neglect with heart rate in delinquent adolescents. J Trauma Stress 17, 47-54

Kop WJ, Krantz DS, Nearing BD, Gottdiener JS, Quigley JF, O'Callahan M, DelNegro AA, Friehling TD, Karasik P, Suchday S, et al. (2004): Effects of acute mental stress and exercise on $\mathrm{T}$-wave alternans in patients with implantable cardioverter defibrillators and controls. Circulation $\underline{109}, 1864-1869$

Kosten TR, Mason JW, Giller EL, Ostroff RB, Harkness L (1987): Sustained urinary norepinephrine and epinephrine elevation in post-traumatic stress disorder. Psychoneuroendocrinology $\underline{12}, 13-20$

Kubzansky LD, Koenen KC, Jones C, Eaton WW (2009): A prospective study of posttraumatic stress disorder symptoms and coronary heart disease in women. Health Psychol $\underline{28}, 125$ 130

Kühner C, Bürger C, Keller F, Hautzinger M (2007): Reliabilität und Validität des revidierten BeckDepressionsinventars (BDI-II): Befunde aus deutschsprachigen Stichproben. Nervenarzt $\underline{78}, 651-656$

La Rovere MT, Mortara A, Schwartz PJ (1995): Baroreflex sensitivity. J Cardiovasc Electrophysiol $\underline{6}, 761-774$

La Rovere MT, Pinna GD, Raczak G (2008): Baroreflex sensitivity: measurement and clinical implications. Ann Noninvasive Electrocardiol 13, 191-207

La Rovere MT, Pinna GD, Maestri R, Robbi E, Caporotondi A, Guazzotti G, Sleight P, Febo O (2009): Prognostic implications of baroreflex sensitivity in heart failure patients in the betablocking era. J Am Coll Cardiol $\underline{53}, 193-199$

Lache B: Beitrag psychometrischer und psychophysiologischer Faktoren zur Vorhersage arrhythmischer Ereignisse bei Patienten mit implantiertem Defibrillator. Med. Diss. Göttingen 2006

Lampe A, Mitmansgruber H, Gast U, Schüssler G, Reddemann L (2008): Therapieevaluation der Psychodynamisch Imaginativen Traumatherapie (PITT) im stationären Setting. Neuropsychiatrie 22, 189-197

Lampe A, Barbist MT, Gast U, Reddemann L, Schüßler G (2014): Long-term course in female survivors of childhood abuse after psychodynamically oriented, trauma-specific inpatient treatment: a naturalistic two-year follow-up. Z Für Psychosom Med Psychother 60, 267282

Lanius RA, Williamson PC, Boksman K, Densmore M, Gupta M, Neufeld RW, Gati JS, Menon RS (2002): Brain activation during script-driven imagery induced dissociative responses in PTSD: a functional magnetic resonance imaging investigation. Biol Psychiatry $\underline{52}$, 305-311

Lanius RA, Bluhm R, Lanius U, Pain C (2006): A review of neuroimaging studies in PTSD: Heterogeneity of response to symptom provocation. J Psychiatr Res $\underline{40}$, 709-729 
Leichsenring F: Borderline-Persönlichkeits-Inventar (BPI): Handanweisung. Hogrefe, Göttingen 1997

Leichsenring F, Chabrol H (2006): Borderline-Persönlichkeits-Inventar (BPI) - ein Selbstbeurteilungs-Instrument zur Erfassung der Borderline-Persönlichkeitsorganisation. PT'T - Persönlichkeitsstörungen Theorie und Therapie $\underline{10}$, 31-42

Leichsenring F, Leibing E, Kruse J, New AS, Leweke F (2011): Borderline personality disorder. Lancet $\underline{377}, 74-84$

Lieb K, Zanarini MC, Schmahl C, Linehan MM, Bohus M (2004): Borderline personality disorder. Lancet $\underline{364}, 453-461$

Light KC, Kothandapani RV, Allen MT (1998): Enhanced cardiovascular and catecholamine responses in women with depressive symptoms. Int J Psychophysiol 28, 157-166

Lindauer RT, van Meijel EP, Jalink M, Olff M, Carlier IV, Gersons BP (2006): Heart rate responsivity to script-driven imagery in posttraumatic stress disorder: Specificity of response and effects of psychotherapy. Psychosom Med $\underline{68}$, 33-40

Lindner M: Borderline-Persönlichkeitsstörung: Eine empirische Untersuchung mit dem BorderlinePersönlichkeits-Inventar. Med. Diss. Göttingen 2001

Linehan MM: Skills training manual for treating borderline personality disorder. Guilford Press, New York 1993

Liu X, Iwanaga K, Shimomura Y, Katsuura T (2007): Comparison of stress responses between mental tasks and white noise exposure. J Physiol Anthropol 26, 165-171

Lomb J, Kleiber C, Herrmann-Lingen C (2015): Stressreaktivität vor und nach integrierter akutpsychosomatischer Komplexbehandlung. Psychother Psychosom Med Psychol 65, 5865

Lomb JM: Physiologische Stressreagibilität vor und nach integrierter akutpsychosomatischer Komplexbehandlung. Med. Diss. Marburg 2011

Macklin ML, Metzger LJ, Lasko NB, Berry NJ, Orr SP, Pitman RK (2000): Five-year follow-up study of eye movement desensitization and reprocessing therapy for combat-related posttraumatic stress disorder. Compr Psychiatry 41, 24-27

Maercker A: IES-R - Impact of Event Scale (Revidierte Form). In: Brähler E, Schumacher J, Strauß B (Hrsg.): Diagnostische Verfahren in der Psychotherapie. Hogrefe, Göttingen 2002, 203 205

Maercker A: Impact of Event Scale - revidierte Form (IES-R). In: Hoyer J, Margraf J (Hrsg.): Angstdiagnostik: Grundlagen und Testverfahren. Springer, Berlin 2003a, 392-395

Maercker A: Therapie der posttraumatischen Belastungsstörungen. 2. Auflage; Springer, Berlin $2003 \mathrm{~b}$

Maercker A: Posttraumatische Belastungsstörungen. 4. Auflage; Springer, Berlin 2013

Maercker A, Schützwohl M (1998): Erfassung von psychischen Belastungsfolgen: Die Impact of Event Skala - revidierte Version (IES-R). Diagnostica 44, 130-141

Malcolm LR: Cardiovascular reactivity in posttraumatic stress disorder and depression. Psych. Diss. Davie 2015 
Matsumura K, Yamakoshi T, Noguchi H, Rolfe P, Matsuoka Y (2012): Fish consumption and cardiovascular response during mental stress. BMC Res Notes $\underline{5}, 288$

Mattheß H, Schüepp R: Dissoziative Störungen. In: Sack M, Sachsse U, Schellong J (Hrsg.): Komplexe Traumafolgestörungen: Diagnostik und Behandlung von Folgen schwerer Gewalt und Vernachlässigung. Schattauer, Stuttgart 2013, 421-436

McDonagh-Coyle A, McHugo GJ, Friedman MJ, Schnurr PP, Zayfert C, Descamps M (2001): Psychophysiological reactivity in female sexual abuse survivors. J Trauma Stress $\underline{14}$, $667-$ 683

McEwen BS (2007): Physiology and neurobiology of stress and adaptation: central role of the brain. Physiol Rev 87, 873-904

McGrath JJ, O’Brien WH, Hassinger HJ, Shah P (2005): Comparability of spot versus band electrodes for impedance cardiography. J Psychophysiol 19, 195-203

Meyer T, Albrecht J, Bornschein G, Sachsse U, Herrmann-Lingen C (2016): Posttraumatic stress disorder (PTSD) patients exhibit a blunted parasympathetic response to an emotional stressor. Appl Psychophysiol Biofeedback $\underline{41}$, 395-404

Mezzacappa ES, Kelsey RM, Katkin ES, Sloan RP (2001): Vagal rebound and recovery from psychological stress. Psychosom Med $\underline{63}, 650-657$

Miller MW, Resick PA (2007): Internalizing and externalizing subtypes in female sexual assault survivors: implications for the understanding of complex PTSD. Behav Ther $\underline{38}, 58-71$

Miller MW, Greif JL, Smith AA (2003): Multidimensional personality questionnaire profiles of veterans with traumatic combat exposure: externalizing and internalizing subtypes. Psychol Assess 15, 205-215

Miller MW, Kaloupek DG, Dillon AL, Keane TM (2004): Externalizing and internalizing subtypes of combat-related PTSD: a replication and extension using the PSY-5 scales. J Abnorm Psychol 113, 636-645

Mitani S, Fujita M, Sakamoto S, Shirakawa T (2006): Effect of autogenic training on cardiac autonomic nervous activity in high-risk fire service workers for posttraumatic stress disorder. J Psychosom Res 60, 439-444

Montoya P, Brody S, Beck K, Veit R, Rau H (1997): Differential $\beta$ - and $\alpha$-adrenergic activation during psychological stress. Eur J Appl Physiol 75, 256-262

Müller S, Sachsse U: Langfristige Chancen und Grenzen in der Traumatherapie: Beobachtungen zum Langzeitverlauf der komplexen posttraumatischen Belastungsstörung (kPTBS). In: Jacobs S (Hrsg.): Neurowissenschaften und Traumatherapie. Univ.-Verl. Göttingen, Göttingen 2009, 83-106

Müller S, Sachsse U (2010): Langzeitverläufe stationärer Psychotherapie bei komplexen Traumafolgestörungen. PTT - Persönlichkeitsstörungen Theorie und Therapie 14, 127-144

Newlin DB, Levenson RW (1979): Pre-ejection period: measuring beta-adrenergic influences upon the heart. Psychophysiology 16, 546-553

Newport DJ, Heim C, Bonsall R, Miller AH, Nemeroff CB (2004): Pituitary-adrenal responses to standard and low-dose dexamethasone suppression tests in adult survivors of child abuse. Biol Psychiatry $\underline{55}, 10-20$ 
Nishith P, Duntley SP, Domitrovich PP, Uhles ML, Cook BJ, Stein PK (2003): Effect of cognitive behavioral therapy on heart rate variability during REM sleep in female rape victims with PTSD. J Trauma Stress 16, 247-250

Oddone AE, Dennis PA, Calhoun PS, Watkins LL, Sherwood A, Dedert EA, Green KT, Stein JN, Dennis MF, Beckham JC (2015): Orthostatic hypotension in young adults with and without posttraumatic stress disorder. Psychol Trauma $\mathbf{7}, 229-233$

Orr SP, Lasko NB, Metzger LJ, Berry NJ, Ahern CE, Pitman RK (1998): Psychophysiologic assessment of women with posttraumatic stress disorder resulting from childhood sexual abuse. J Consult Clin Psychol 66, 906-913

Ottaviani C, Shapiro D, Goldstein IB, James JE, Weiss R (2006): Hemodynamic profile, compensation deficit, and ambulatory blood pressure. Psychophysiology $\underline{43}$, 46-56

Ottaviani C, Shapiro D, Davydov DM, Goldstein IB (2008): Autonomic stress response modes and ambulatory heart rate level and variability. J Psychophysiol 22, 28-40

Paine NJ, Ring C, Bosch JA, McIntyre D, Veldhuijzen van Zanten JJ (2013): The effect of acute mental stress on limb vasodilation is unrelated to total peripheral resistance.

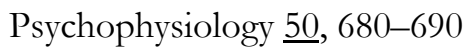

Panksepp J: Affective neuoscience: The foundations of human and animal emotions. Oxford University Press, New York 1998

Paris J: Aversive Kindheitserlebnisse und Borderline-Persönlichkeitsstörung. In: Dulz B, Herpertz S, Kernberg O, Sachsse U (Hrsg.): Handbuch der Borderline-Störungen. 2. Auflage; Schattauer, Stuttgart 2011, 192-196

Paulus EJ, Argo TR, Egge JA (2013): The impact of posttraumatic stress disorder on blood pressure and heart rate in a veteran population. J Trauma Stress $\underline{26}, 169-172$

Peckerman A, Dahl K, Chemitiganti R, LaManca JJ, Ottenweller JE, Natelson BH (2003): Effects of posttraumatic stress disorder on cardiovascular stress responses in Gulf War veterans with fatiguing illness. Auton Neurosci Basic Clin $\underline{108}$, 63-72

Peñáz J, Voigt A, Teichmann W (1976): Beitrag zur fortlaufenden indirekten Blutdruckmessung. Z Gesamte Inn Med 1ㅜ 1030-1033

Pervanidou P (2008): Biology of post-traumatic stress disorder in childhood and adolescence. J Neuroendocrinol 20, 632-638

Phillips AC, Hunt K, Der G, Carroll D (2011): Blunted cardiac reactions to acute psychological stress predict symptoms of depression five years later: Evidence from a large community study. Psychophysiology $\underline{48}, 142-148$

Pineles SL, Suvak MK, Liverant GI, Gregor K, Wisco BE, Pitman RK, Orr SP (2013): Psychophysiologic reactivity, subjective distress, and their associations with PTSD diagnosis. J Abnorm Psychol 122, 635-644

Pinna GD, Maestri R, Capomolla S, Febo O, Robbi E, Cobelli F, La Rovere MT (2005): Applicability and clinical relevance of the transfer function method in the assessment of baroreflex sensitivity in heart failure patients. J Am Coll Cardiol 46, 1314-1321

Pole N (2007): The psychophysiology of posttraumatic stress disorder: A meta-analysis. Psychol Bull 133, 725-746 
Raaijmakers E, Faes TJ, Scholten RJ, Goovaerts HG, Heethaar RM (1999): A meta-analysis of three decades of validating thoracic impedance cardiography. Crit Care Med June 1999 27, 1203 1213

Rabe S, Dörfel D, Zöllner T, Maercker A, Karl A (2006): Cardiovascular correlates of motor vehicle accident related posttraumatic stress disorder and its successful treatment. Appl Psychophysiol Biofeedback $\underline{31}, 315-330$

Reddemann L: Psychodynamisch Imaginative Traumatherapie: PITT - das Manual. Klett-Cotta, Stuttgart 2011

Reijman S, Alink LR, Compier-de Block LH, Werner CD, Maras A, Rijnberk C, van Ijzendoorn MH, Bakermans-Kranenburg MJ (2014): Autonomic reactivity to infant crying in maltreating mothers. Child Maltreat 19, 101-112

Resick PA, Bovin MJ, Calloway AL, Dick AM, King MW, Mitchell KS, Suvak MK, Wells SY, Stirman SW, Wolf EJ (2012): A critical evaluation of the complex PTSD literature: implications for DSM-5. J Trauma Stress $\underline{25}$, 241-251

Richter P: Zur Konstruktvalidität des Beck-Depressionsinventars (BDI) bei der Erfassung depressiver Verläufe: ein empirischer und methodologischer Beitrag. Roderer, Regensburg 1991

Richter P, Werner J, Heerlein A, Kraus A, Sauer H (1998): On the validity of the Beck Depression Inventory. A review. Psychopathology $\underline{31}, 160-168$

Riem MM, Pieper S, Out D, Bakermans-Kranenburg MJ, van Ijzendoorn MH (2011): Oxytocin receptor gene and depressive symptoms associated with physiological reactivity to infant crying. Soc Cogn Affect Neurosci $\underline{6}, 294-300$

Ring C, Harrison LK, Winzer A, Carroll D, Drayson M, Kendall M (2000): Secretory immunoglobulin A and cardiovascular reactions to mental arithmetic, cold pressor, and exercise: Effects of alpha-adrenergic blockade. Psychophysiology 37, 634-643

Ring C, Burns VE, Carroll D (2002): Shifting hemodynamics of blood pressure control during prolonged mental stress. Psychophysiology $\underline{39}, 585-590$

Rodewald F, Gast U, Emrich HM (2006): Screening auf Komplexe Dissoziative Störungen mit dem Fragebogen für dissoziative Symptome (FDS). Psychother Psychosom Med Psychol $\underline{56}$, 249-258

Sachsse U (2003): Distress-Systeme des Menschen. PTT - Persönlichkeitsstörungen Theorie und Therapie $\mathbf{7}, 4-15$

Sachsse U: Traumazentrierte Psychotherapie. Schattauer, Stuttgart 2004

Sachsse U, Schröder S, Vogel C: Klinisch erfolgreich - Empirisch erfolgreich? In: Sachsse U, Özkan I, Streeck-Fischer A (Hrsg.): Traumatherapie - was ist erfolgreich? Vandenhoeck \& Ruprecht, Göttingen 2002, 39-53

Sachsse U, Vogel C, Leichsenring F (2006): Results of psychodynamically oriented trauma-focused inpatient treatment for women with complex posttraumatic stress disorder (PTSD) and borderline personality disorder (BPD). Bull Menninger Clin $\underline{70}$, 125-144

Sack M (2004a): Diagnostische und klinische Aspekte der komplexen posttraumatischen Belastungsstörung. Nervenarzt $\underline{75}$, 451-459 
Sack M: Psychophysiologische Reaktionen bei Konfrontation mit einer traumatischen Erinnerung der Einfluss akuter dissoziativer Symptome auf die autonom-vegetative Regulation. Med. Habil.-Schr. Hannover 2004b

Sack M, Hopper JW, Lamprecht F (2004): Low respiratory sinus arrhythmia and prolonged psychophysiological arousal in posttraumatic stress disorder: heart rate dynamics and individual differences in arousal regulation. Biol Psychiatry $\underline{55}$, 284-290

Sack M, Sachsse U, Dulz B: Ist die Borderline-Persönlichkeitsstörung eine Traumafolgestörung? In: Dulz B, Herpertz SC, Kernberg OF, Sachsse U (Hrsg.): Handbuch der BorderlineStörungen. 2. Auflage; Schattauer, Stuttgart 2011, 197-202

Sack M, Cillien M, Hopper JW (2012): Acute dissociation and cardiac reactivity to script-driven imagery in trauma-related disorders. Eur J Psychotraumatol $\underline{3}, 17419$

Sack M, Sachsse U, Overkamp B, Dulz B (2013): Traumafolgestörungen bei Patienten mit Borderline-Persönlichkeitsstörung. Nervenarzt $\underline{84}, 608-614$

Sahar T, Shalev AY, Porges SW (2001): Vagal modulation of responses to mental challenge in posttraumatic stress disorder. Biol Psychiatry $\underline{49}$, 637-643

Salomon K, Clift A, Karlsdóttir M, Rottenberg J (2009): Major depressive disorder is associated with attenuated cardiovascular reactivity and impaired recovery among those free of cardiovascular disease. Health Psychol 28, 157-165

Sammito S, Thielmann B, Zimmermann P, Böckelmann I (2015): Einfluss einer posttraumatischen Belastungsstörung auf die Herzfrequenzvariabilität als Marker des autonomen Nervensystems - eine systematische Literaturübersicht. Fortschr Neurol Psychiatr $\underline{83}, 30$ 37

Sautter FJ, Bissette G, Wiley J, Manguno-Mire G, Schoenbachler B, Myers L, Johnson JE, Cerbone A, Malaspina D (2003): Corticotropin-releasing factor in posttraumatic stress disorder (PTSD) with secondary psychotic symptoms, nonpsychotic PTSD, and healthy control subjects. Biol Psychiatry $\underline{54}, 1382-1388$

Schellong J: Diagnostische Klassifikation von Traumafolgestörungen. In: Sack M, Sachsse U, Schellong J (Hrsg.): Komplexe Traumafolgestörungen: Diagnostik und Behandlung von Folgen schwerer Gewalt und Vernachlässigung. Schattauer, Stuttgart 2013, 42-58

Schmitz N, Hartkamp N, Kiuse J, Franke GH, Reister G, Tress W (2000): The Symptom CheckList-90-R (SCL-90-R): a German validation study. Qual Life Res 9, 185-193

Sellbom M, Bagby RM (2009): Identifying PTSD personality subtypes in a workplace trauma sample. J Trauma Stress $\underline{22}, 471-475$

Shalev AY, Orr SP, Pitman RK (1992): Psychophysiologic response during script-driven imagery as an outcome measure in posttraumatic stress disorder. J Clin Psychiatry $\underline{53}$, 324-326

Shapiro F: EMDR - Grundlagen \& Praxis. 2. Auflage; Junfermann, Paderborn 1999

Shapiro F (2014): The role of eye movement desensitization and reprocessing (EMDR) therapy in medicine: Addressing the psychological and physical symptoms stemming from adverse life experiences. Perm J $\underline{18}, 71-77$

Sherwood A, Johnson K, Blumenthal JA, Hinderliter AL (1999): Endothelial function and hemodynamic responses during mental stress. Psychosom Med 61, 365-370 
Steer RA, Beck AT, Garrison B: Applications of the Beck Depression Inventory. In: Sartorius N, Ban TA (Hrsg.): Assessment of Depression. Springer, Berlin 1986, 123-142

Steil R, Dyer A, Priebe K, Kleindienst N, Bohus M (2011): Dialectical behavior therapy for posttraumatic stress disorder related to childhood sexual abuse: A pilot study of an intensive residential treatment program. J Trauma Stress 24, 102-106

Stein MB, Yehuda R, Koverola C, Hanna C (1997): Enhanced dexamethasone suppression of plasma cortisol in adult women traumatized by childhood sexual abuse. Biol Psychiatry $\underline{42}$, 680-686

Steptoe A, Sawada Y (1989): Assessment of baroreceptor reflex function during mental stress and relaxation. Psychophysiology 26, 140-147

Tan G, Dao TK, Farmer L, Sutherland RJ, Gevirtz R (2011): Heart rate variability (HRV) and posttraumatic stress disorder (PTSD): A pilot study. Appl Psychophysiol Biofeedback $\underline{36}$, $27-35$

Terr LC (1991): Childhood traumas: an outline and overview. Am J Psychiatry 148, 10-20

Tritt K, von Heymann F, Konermann J, Bidmon RK, Nickel M, Götz K, Loew T, Zaudig M (2006): Der Einsatz der Symptom Checkliste SCL-90-R bei Persönlichkeitsstörungen. PTT - Persönlichkeitsstörungen Theorie und Therapie 10, 18-30

Tsai P, Yucha CB, Nichols WW, Yarandi H (2003): Hemodynamics and arterial properties in response to mental stress in individuals with mild hypertension. Psychosom Med $\underline{65}$, 613619

Tye S, Van Voorhees E, Hu C, Lineberry T (2015): Preclinical perspectives on posttraumatic stress disorder criteria in DSM-5. Harv Rev Psychiatry 23, 51-58

Van der Kolk BA (2006): Clinical implications of neuroscience research in PTSD. Ann N Y Acad Sci $\underline{1071}, 277-293$

Van Ijzendoorn MH, Schuengel C (1996): The measurement of dissociation in normal and clinical populations: Meta-analytic validation of the Dissociative Experiences Scale (DES). Clin Psychol Rev 16, 365-382

Van 't Wout M, Spofford CM, Unger WS, Sevin EB, Shea MT (2017): Skin conductance reactivity to standardized virtual reality combat scenes in veterans with PTSD. Appl Psychophysiol Biofeedback

Vrana SR, Hughes JW, Dennis MF, Calhoun PS, Beckham JC (2009): Effects of posttraumatic stress disorder status and covert hostility on cardiovascular responses to relived anger in women with and without PTSD. Biol Psychol 2, 274-280

Wangelin BC, Tuerk PW (2015): Taking the pulse of prolonged exposure therapy: Physiological reactivity to trauma imagery as an objective measure of treatment response. Depress Anxiety $\underline{32}, 927-934$

Weiss DS, Marmar CR: The Impact of Event Scale - Revised. In: Wilson JP, Keane TM (Hrsg.): Assessing psychological trauma and PTSD. Guilford Press, New York 1997, 399-411

WHO: Internationale Klassifikation psychischer Störungen - ICD-10 Kapitel V (F) Klinischdiagnostische Leitlinien. Hrsg. v. Dilling H, Mombour W, Schmidt MH, 9. Auflage; Verlag Hans Huber, Bern 2014 
Winzer A, Ring C, Carroll D, Willemsen G, Drayson M, Kendall M (1999): Secretory immunoglobulin A and cardiovascular reactions to mental arithmetic, cold pressor, and exercise: Effects of beta-adrenergic blockade. Psychophysiology 36, 591-601

Wise K: Entwicklung und Evaluierung einer Gruppentherapie für sexuell-missbrauchte Frauen. 2. Jahrestagung der Deutschsprachigen Gesellschaft für Psychotraumatologie. Abstractband; Klinik für Psychosomatik und psychotherapeutische Medizin, Aachen 2000, 16

Woon FL, Sood S, Hedges DW (2010): Hippocampal volume deficits associated with exposure to psychological trauma and posttraumatic stress disorder in adults: a meta-analysis. Prog Neuropsychopharmacol Biol Psychiatry $\underline{34}, 1181-1188$

Xie L, Liu B, Wang X, Mei M, Li M, Yu X, Zhang J (2017): Effects of different stresses on cardiac autonomic control and cardiovascular coupling. J Appl Physiol 122, 435-445

Xue Y, Taub PR, Iqbal N, Fard A, Wentworth B, Redwine L, Clopton P, Stein M, Maisel A (2012): Cardiac biomarkers, mortality, and post-traumatic stress disorder in military veterans. Am J Cardiol $\underline{109}, 1215-1218$

Yehuda R: Die Neuroendokrinologie bei Posttraumatischer Belastungsstörung im Licht neuer neuroanatomischer Befunde. In: Streeck-Fischer A, Sachsse U, Özkan I (Hrsg.): Körper, Seele, Trauma - Biologie, Klinik und Praxis. Vandenhoeck \& Ruprecht, Göttingen 2001, $43-71$

Yehuda R (2009): Status of glucocorticoid alterations in post-traumatic stress disorder. Ann N Y Acad Sci 1179, 56-69

Yehuda R, Siever LJ, Teicher MH, Levengood RA, Gerber DK, Schmeidler J, Yang RK (1998): Plasma norepinephrine and 3-methoxy-4-hydroxyphenylglycol concentrations and severity of depression in combat posttraumatic stress disorder and major depressive disorder. Biol Psychiatry $\underline{44}, 56-63$

Yehuda R, Halligan SL, Grossman R, Golier JA, Wong C (2002): The cortisol and glucocorticoid receptor response to low dose dexamethasone administration in aging combat veterans and holocaust survivors with and without posttraumatic stress disorder. Biol Psychiatry $\underline{52}$, 393-403

Yehuda R, Golier JA, Yang RK, Tischler L (2004): Enhanced sensitivity to glucocorticoids in peripheral mononuclear leukocytes in posttraumatic stress disorder. Biol Psychiatry $\underline{55}$, 1110-1116

Yen S, Shea MT, Battle CL, Johnson DM, Zlotnick C, Dolan-Sewell R, Skodol AE, Grilo CM, Gunderson JG, Sanislow CA, et al. (2002): Traumatic exposure and posttraumatic stress disorder in borderline, schizotypal, avoidant, and obsessive-compulsive personality disorders: findings from the collaborative longitudinal personality disorders study. J Nerv Ment Dis $\underline{190}, 510-518$

York KM, Hassan M, Li Q, Li H, Fillingim RB, Sheps DS (2007): Coronary artery disease and depression: Patients with more depressive symptoms have lower cardiovascular reactivity during laboratory-induced mental stress. Psychosom Med 69, 521-528

Zanarini MC (2000): Childhood experiences associated with the development of borderline personality disorder. Psychiatr Clin North Am 23, 89-101

Zanarini MC, Williams AA, Lewis RE, Reich RB, Vera SC, Marino MF, Levin A, Yong L, Frankenburg FR (1997): Reported pathological childhood experiences associated with the development of borderline personality disorder. Am J Psychiatry 154, 1101-1106 
Zanarini MC, Yong L, Frankenburg FR, Hennen J, Reich DB, Marino MF, Vujanovic AA (2002): Severity of reported childhood sexual abuse and its relationship to severity of borderline psychopathology and psychosocial impairment among borderline inpatients. J Nerv Ment Dis $190,381-387$

Zehl S, Sachsse U, Dulz B, Overkamp B, Sack M (2013): Internalisierung und Externalisierung als Ausprägungen einer gemeinsamen Störungsdimension bei traumatisierten Patienten. Psychother Psychosom Med Psychol 63, 429-438

Zucker TL, Samuelson KW, Muench F, Greenberg MA, Gevirtz RN (2009): The effects of respiratory sinus arrhythmia biofeedback on heart rate variability and posttraumatic stress disorder symptoms: A pilot study. Appl Psychophysiol Biofeedback $\underline{34}$, 135-143 


\section{Danksagung}

An dieser Stelle möchte ich mich bei all jenen bedanken, die mich bei der Durchführung des Follow-ups und der Anfertigung der Doktorarbeit unterstützt haben und ohne deren Mithilfe die Erstellung dieser Arbeit nicht möglich gewesen wäre.

Zunächst und in besonderem Maße danke ich Herrn Professor Herrmann-Lingen für die freundliche Überlassung des überaus interessanten Themas, für die fachliche und engagierte Betreuung sowie für die stets wertvollen Ratschläge. Das konstruktive Feedback hat mir sehr geholfen und zu einer intensiven Auseinandersetzung mit der Thematik beigetragen. Auch möchte ich mich bei Herrn Professor Sachsse bedanken, durch dessen Einsatz die Rekrutierung der Studienteilnehmerinnen ermöglicht wurde. Mit seiner Expertise im Bereich der komplexen Traumafolgestörungen war er ein wichtiger Ansprechpartner für mich - dies war insbesondere für den Kontakt mit dieser besonderen Patientengruppe sehr hilfreich. Mein Dank gilt zudem Frau Dr. Chavanon, die mir in Hinblick auf die statistische Auswertung stets unterstützend und kompetent zur Seite stand.

Ein besonderer Dank ist an all die Probandinnen der Studie gerichtet. Hierbei möchte ich insbesondere die Follow-up-Teilnehmerinnen hervorheben, denn ihre Teilnahme war nicht selten mit einer langen Anreise und einem erheblichen Stress verbunden. Nur durch die bemerkenswerte Motivation der Probandinnen, einen Beitrag zur Forschung an der Psychophysiologie der komplexen Traumafolgestörungen zu leisten, wurde dieses Follow-up und damit auch diese Doktorarbeit erst möglich. 